\title{
Fetal Electrocardiogram Extraction and Enhancement using Triggered Adaptive Filtering
}

by

\author{
Vesal Badee \\ A thesis \\ presented to Carleton University \\ in partial fulfillment of the requirements for the degree of \\ Master of Applied Science \\ in \\ Electrical Engineering
}

Ottawa, Ontario, Canada, 2006

CVesal Badee, 2006 


$\begin{array}{ll}\begin{array}{l}\text { Library and } \\ \text { Archives Canada }\end{array} & \begin{array}{l}\text { Bibliothèque et } \\ \text { Archives Canada }\end{array} \\ \begin{array}{l}\text { Published Heritage } \\ \text { Branch }\end{array} & \begin{array}{l}\text { Direction du } \\ \text { Patrimoine de l'édition }\end{array} \\ \begin{array}{l}\text { 395 Wellington Street } \\ \text { Ottawa ON K1A ON4 }\end{array} & \begin{array}{l}\text { 395, rue Wellington } \\ \text { Ottawa ON K1A ON4 } \\ \text { Canada }\end{array}\end{array}$

Your file Votre référence ISBN: 978-0-494-23326-9 Our file Notre référence ISBN: 978-0-494-23326-9

NOTICE:

The author has granted a nonexclusive license allowing Library and Archives Canada to reproduce, publish, archive, preserve, conserve, communicate to the public by telecommunication or on the Internet, loan, distribute and sell theses worldwide, for commercial or noncommercial purposes, in microform, paper, electronic and/or any other formats.

The author retains copyright ownership and moral rights in this thesis. Neither the thesis nor substantial extracts from it may be printed or otherwise reproduced without the author's permission.
AVIS:

L'auteur a accordé une licence non exclusive permettant à la Bibliothèque et Archives Canada de reproduire, publier, archiver, sauvegarder, conserver, transmettre au public par télécommunication ou par l'Internet, prêter, distribuer et vendre des thèses partout dans le monde, à des fins commerciales ou autres, sur support microforme, papier, électronique et/ou autres formats.

L'auteur conserve la propriété du droit d'auteur et des droits moraux qui protège cette thèse. $\mathrm{Ni}$ la thèse ni des extraits substantiels de celle-ci ne doivent être imprimés ou autrement reproduits sans son autorisation.
In compliance with the Canadian

Privacy Act some supporting forms may have been removed from this thesis.

While these forms may be included in the document page count, their removal does not represent any loss of content from the thesis.
Conformément à la loi canadienne sur la protection de la vie privée, quelques formulaires secondaires ont été enlevés de cette thèse.

Bien que ces formulaires aient inclus dans la pagination, il n'y aura aucun contenu manquant.

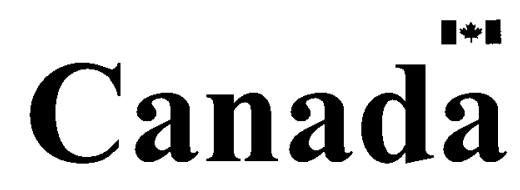




\section{Author's Declaration}

I hereby declare that I am the sole author of this thesis.

I authorize Carleton University to lend this thesis to other institutions or individuals for the purpose of scholarly research.

Signature

I further authorize Carleton University to reproduce this thesis by photocopying or by other means, in total or in part, at the request of other institutions or individuals for the purpose of scholarly research.

Signature

iii 


\section{Abstract}

Analysis of the electrocardiogram (ECG) is a simple and noninvasive method of effectively assessing a patient's general health and diagnosing cardiovascular diseases. The ECG can also be used to clinically evaluate the health of prenatal infants. Variations in the FECG waveform morphology can provide important diagnostic information that is not available in current fetal monitoring techniques, such as ultrasound and phonocardiography. Such evaluations offer the opportunity to combat prenatal health conditions sooner, and offer prenatal awareness of potential physical abnormalities and complications.

The objective of this thesis is to investigate a novel multichannel and multimodal FECG extraction (stage 1) and enhancement (stage 2) technique using linear adaptive filtering with triggering, based on noninvasive abdominal measurements. In stage 1, we show that by controlling the periods of adaptation, a more accurate FECG signal can be extracted. The concept of triggering is proposed with the inclusion of an extra modality, such as the fetal heart sound or Doppler ultrasound data, to be combined with the electrical modality of the ECG, to provide temporal information about the FECG. Using synthetic ECG data, our results indicate a $5.49 \%$ PPRD improvement using triggering with linear transitions compared to no triggering. Slight improvements were obtained with triggering compared to no triggering with simulated FECG data and real data.

In stage 2, we investigate the extension of triggering to FECG enhancement using multiple abdominal channels. Our results indicate an increase in the $S_{N R}$ est 2.61 using triggering with linear transitions over no triggering with simulated FECG data, and an increase of 0.81 using triggering with on/off transitions over no triggering with real data. 


\section{Acknowledgements}

I would like to thank my supervisors, Dr. Adrian Chan and Dr. Richard Dansereau, for their dedication, support and invaluable contributions throughout my studies. They have been readily available to assist me with problems, recommend solutions and edit my work with excellent attention to detail. Their professionalism and expertise has made them role models to me, and I cannot thank them enough.

I would like to acknowledge my good friends and fellow engineers: Nabil Yazdani, Hua Jin Guo, Geoff Green and Mehran Talebinejad. I have really enjoyed working with them, and my experience here at Carleton University wouldn't have been the same without them.

Last, but certainly not least, I would like to thank my family. They have supported and encouraged me, and I will be forever grateful to them. 


\section{Table of Contents}

Author's Declaration.............................................................................................................ii

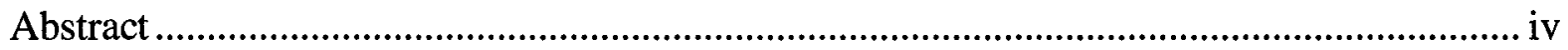

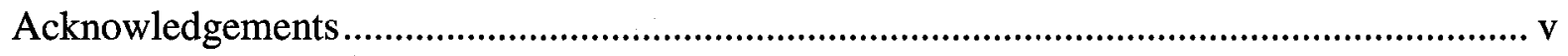

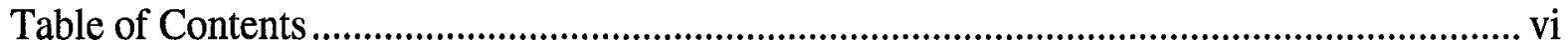

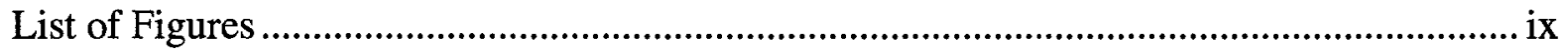

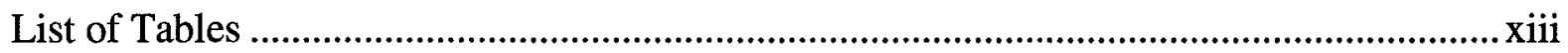

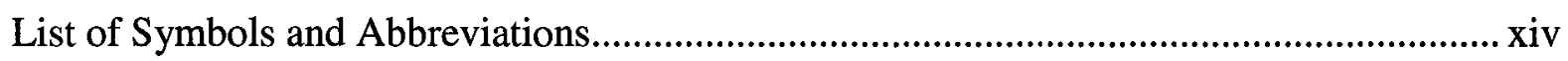

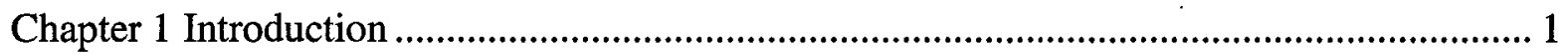

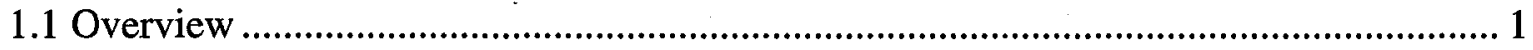

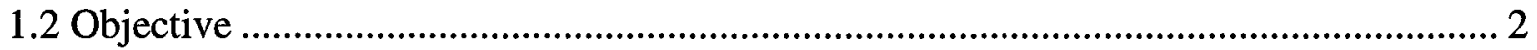

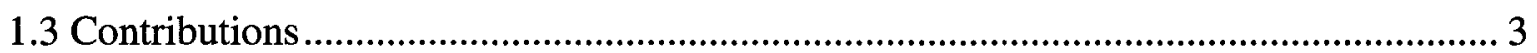

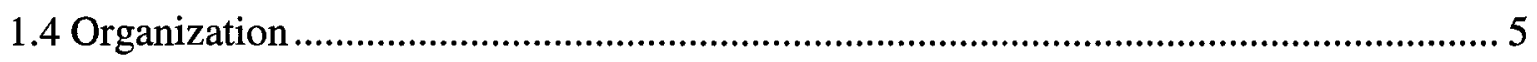

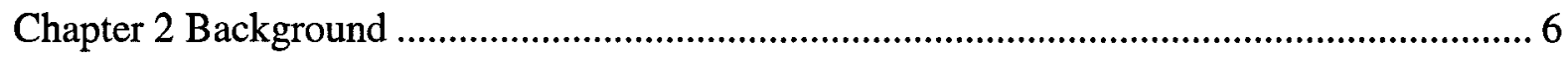

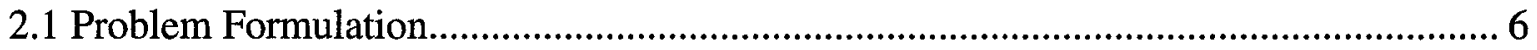

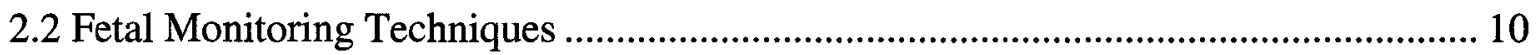

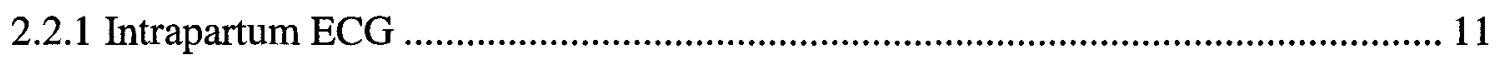

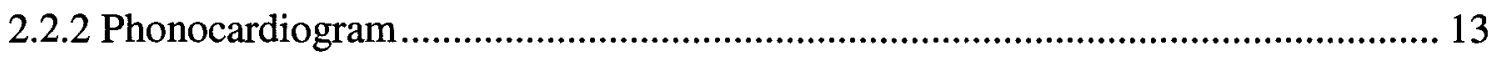

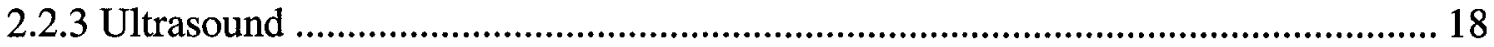

2.3 Fetal ECG Extraction using Adaptive Noise Cancellation ...................................... 19

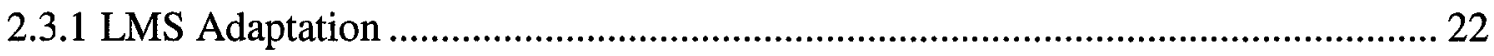

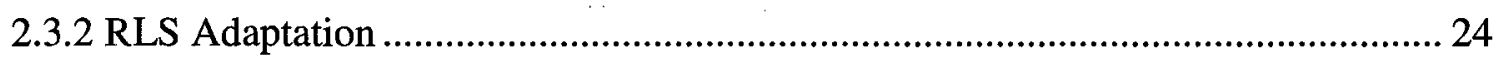

2.4 Fetal ECG Extraction using Blind Source Separation ............................................ 26

Chapter 3 Fetal ECG Extraction using Triggered Adaptive Noise Cancellation................... 28

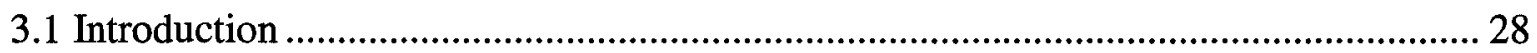

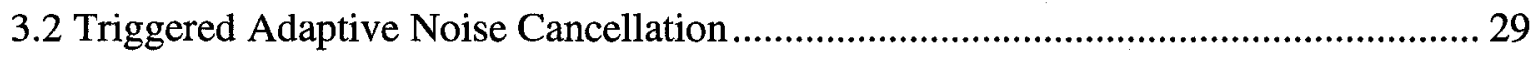

3.2.1 Fetal Phonocardiogram Trigger ..................................................................... 30

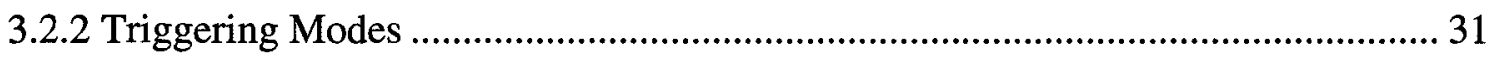

3.2.3 Weighted Transitions.............................................................................. 34

vi 


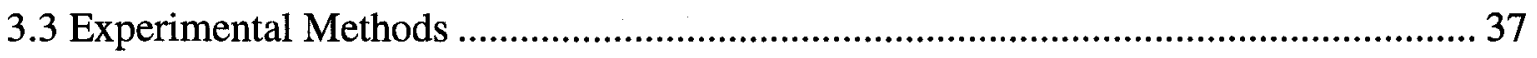

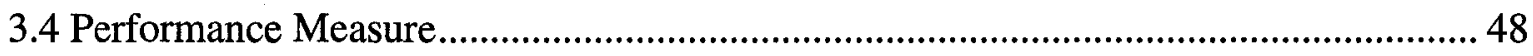

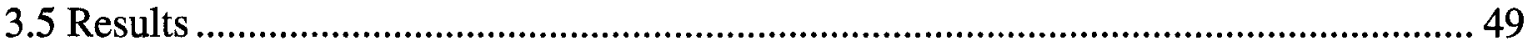

3.5.1 Effect of MECG Trigger Window Size .................................................................. 54

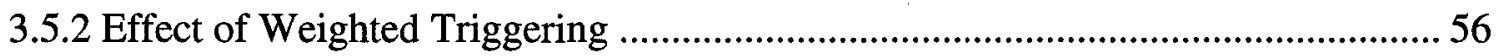

3.5.3 Effect of Lowering the SNR of the Synthetic Data .............................................59

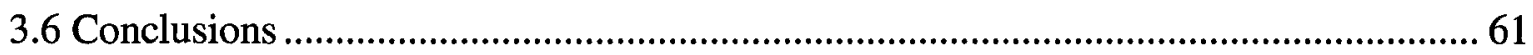

Chapter 4 Multichannel Fetal ECG Extraction and Enhancement .................................. 63

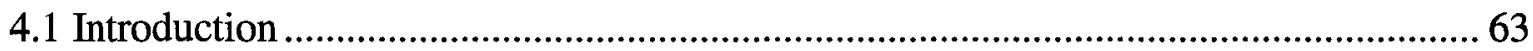

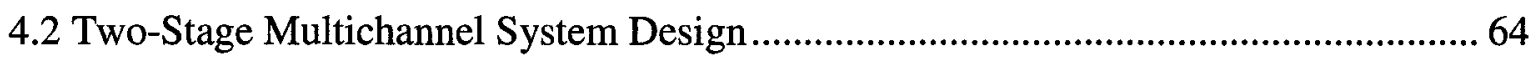

4.2.1 Stage 1: Fetal ECG Extraction using Triggered Adaptive Noise Cancellation...... 64

4.2.2 Stage 2: Fetal ECG Enhancement using Triggered Adaptive System Identification 66

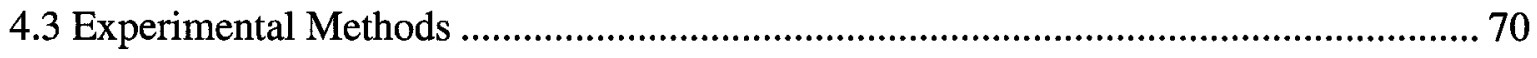

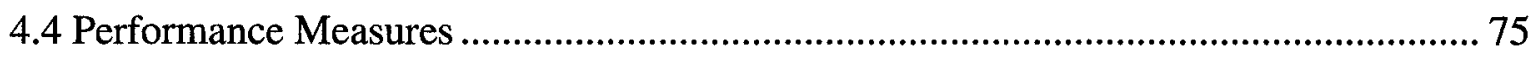

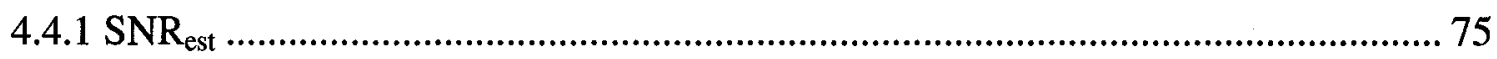

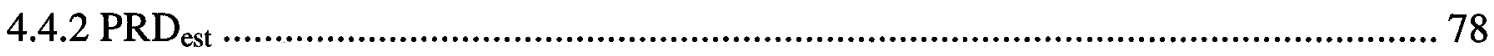

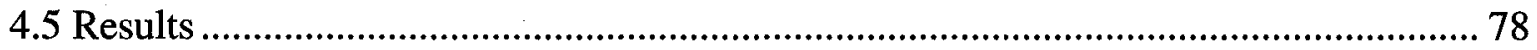

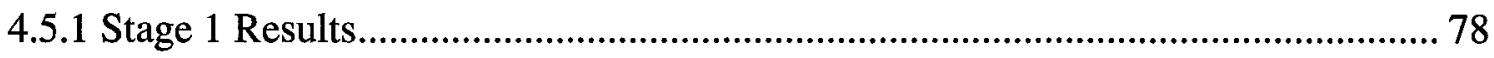

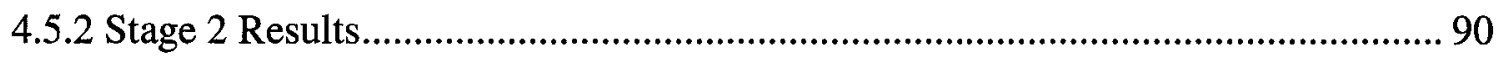

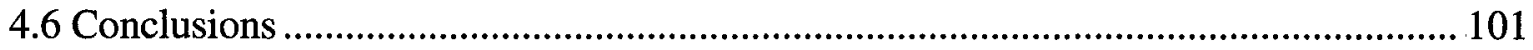

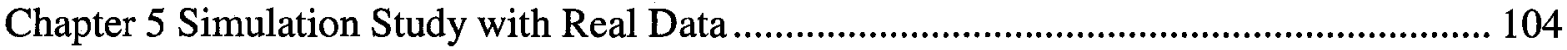

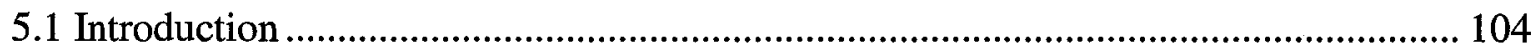

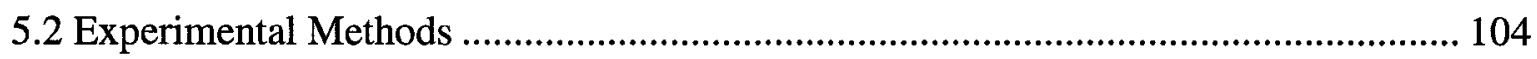

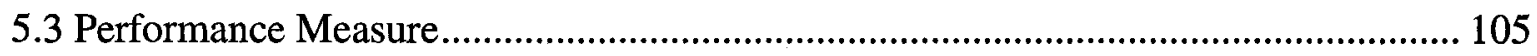

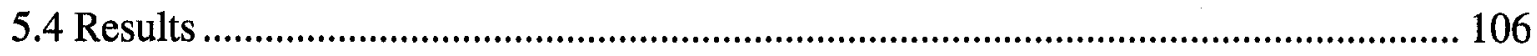

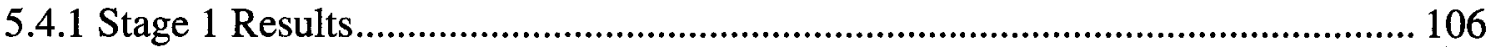

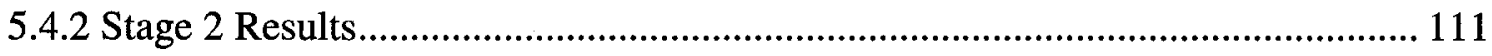

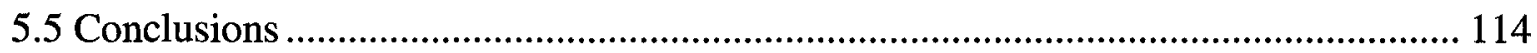

vii 


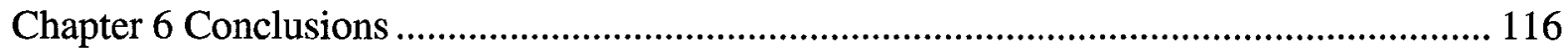

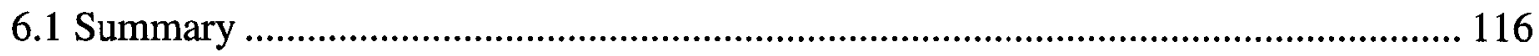

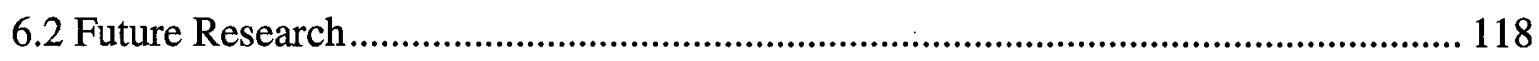

Appendix A Carleton University Research Ethics Committee Consent Form .................... 121 


\section{List of Figures}

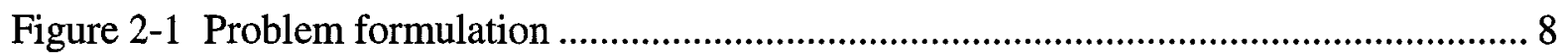

Figure 2-2 Simplified problem formulation.................................................................

Figure 2-3 Normal electrocardiogram ........................................................................... 11

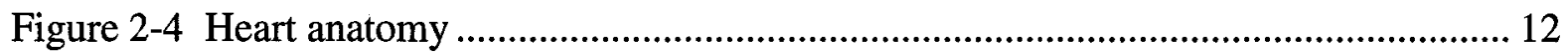

Figure 2-5 Normal phonocardiogram ............................................................................... 14

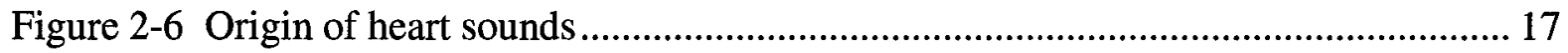

Figure 2-7 Adaptive noise cancellation scheme applied to FECG extraction ........................ 20

Figure 3-1 Triggered adaptive noise cancellation applied to FECG extraction...................... 30

Figure 3-2 Correlation of the ECG and the PCG ................................................................. 31

Figure 3-3 Triggering Modes............................................................................................ 33

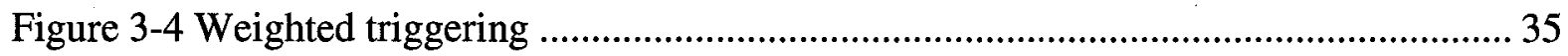

Figure 3-5 Three-dimensional state-space synthetic ECG..................................................... 39

Figure 3-6 Two-dimensional synthetic temporal ECG........................................................... 41

Figure 3-7 Data acquisition electrode placement.................................................................. 43

Figure 3-8 System identification problem ..................................................................... 44

Figure 3-9 Magnitude of the frequency response between the thoracic ECG and the abdominal ECG in a subject ................................................................................................ 45

Figure 3-10 Synthetic $M E C G_{t}$ data with AWGN................................................................ 46

Figure 3-11 Synthetic $M E C G_{a}$ data with AWGN ………….................................................. 47

Figure 3-12 Synthetic $F E C G_{a}$ data with AWGN ............................................................... 47

Figure 3-13 Synthetic $C E C G$ data with AWGN.................................................................. 48

Figure 3-14 Power spectral density of the ideal synthetic FECG ............................................50

Figure 3-15 Effect of filter order on the average pulse PRD using the LMS and RLS algorithms with no triggering............................................................................................... 51

Figure 3-16 Effect of filter order on the average pulse PRD for various triggering modes using the RLS algorithm ............................................................................................ 52

Figure 3-17 (a) Ideal FECG, and (b) FECG estimates.......................................................53 
Figure 3-18 Effect of MECG trigger window size on the average pulse PRD. 55

Figure 3-19 Effect of $m_{t}$ on the FECG estimate ............................................................56

Figure 3-20 Linear transition triggering with maximum $m_{t}$ constraint ............................58

Figure 3-21 (a) Ideal FECG, and (b) FECG estimates..................................................59

Figure 3-22 Effect of the abdominal composite SNR on the FECG estimate ....................60

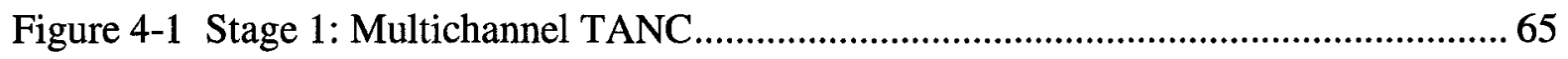

Figure 4-2 Triggered adaptive system identification applied to FECG enhancement.......... 67

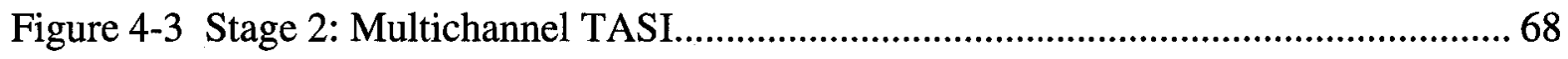

Figure 4-4 Two-stage multichannel signal extraction ...................................................69

Figure 4-5 Data acquisition and stimulus delivery electrode placements on front and back of

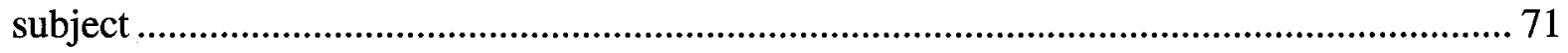

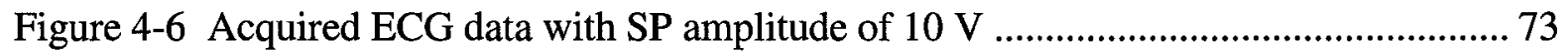

Figure 4-7 Acquired ECG data with SP amplitude of $12 \mathrm{~V}$.............................................. 73

Figure 4-8 Acquired ECG data with SP amplitude of $14 \mathrm{~V}$.......................................... 74

Figure 4-9 Acquired ECG data with SP amplitude of $20 \mathrm{~V} \mathrm{\ldots ........................................} 74$

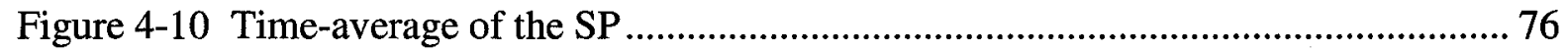

Figure 4-11 SNR $_{\text {est }}$ of abdominal channels after ANC for various filter orders using an

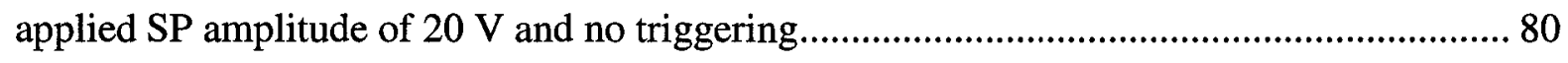

Figure 4-12 $\mathrm{PRD}_{\text {est }}$ of abdominal channels after ANC for various filter orders using an

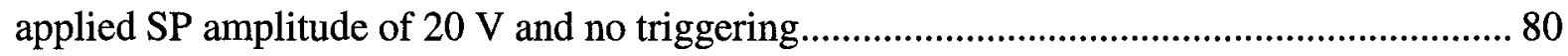

Figure 4-13 Triggering modes used for TANC …....................................................... 81

Figure 4-14 SNR $_{\text {est }}$ of abdominal channels after TANC for various triggering modes and an

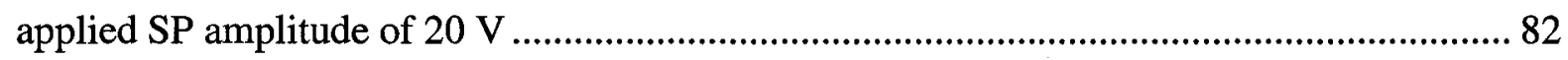

Figure 4-15 $\mathrm{PRD}_{\mathrm{est}}$ of abdominal channels after TANC for various triggering modes and an

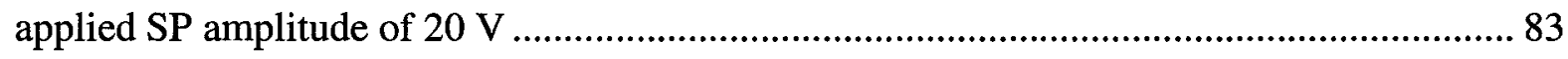

Figure 4-16 Estimated SP after TANC using channel 2 for various triggering modes with an applied SP amplitude of $20 \mathrm{~V}$

Figure 4-17 $\mathrm{SNR}_{\text {est }}$ of abdominal channels after TANC for various triggering modes with an applied SP amplitude of $14 \mathrm{~V}$ 85 
Figure 4-18 $\mathrm{PRD}_{\text {est }}$ of abdominal channels after TANC for various triggering modes with an applied SP amplitude of $14 \mathrm{~V}$ 86

Figure 4-19 $\mathrm{SNR}_{\text {est }}$ of abdominal channels after TANC for various triggering modes with an applied SP amplitude of $12 \mathrm{~V}$ 86

Figure 4-20 $\mathrm{PRD}_{\text {est }}$ of abdominal channels after TANC for various triggering modes with an applied SP amplitude of $12 \mathrm{~V}$

Figure 4-21 SNR $_{\text {est }}$ of abdominal channels after TANC for various triggering modes with an applied SP amplitude of $10 \mathrm{~V}$ 87

Figure 4-22 $\mathrm{PRD}_{\text {est }}$ of abdominal channels after TANC for various triggering modes with an applied SP amplitude of $10 \mathrm{~V}$ 88

Figure 4-23 SNR $_{\text {est }}$ after TANC for various applied SP amplitudes using channel 2 89

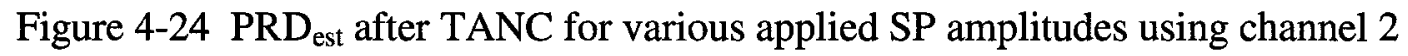
89

Figure 4-25 SNR $_{\text {est }}$ of SP estimate after ASI for various filter orders with an applied SP amplitude of $20 \mathrm{~V}$ and no triggering

Figure 4-26 PRD est $_{\text {of }}$ SP estimate after ASI for various filter orders with an applied SP

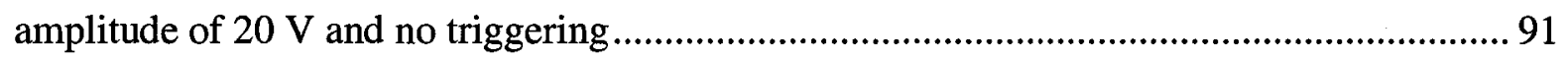

Figure 4-27 Triggering modes used for TASI ............................................................. 92

Figure 4-28 SNR $_{\text {est }}$ of final estimate after TASI for various triggering modes with an applied SP amplitude of $20 \mathrm{~V}$

Figure 4-29 $\mathrm{PRD}_{\text {est }}$ of final estimate after TASI for various triggering modes with an applied SP amplitude of $20 \mathrm{~V}$ .94

Figure 4-30 Estimated SP after TANC and TASI for various triggering modes with an applied SP amplitude of $20 \mathrm{~V}$

Figure 4-31 SNR $_{\text {est }}$ of final estimate after TASI for various triggering modes and an applied SP amplitude of $14 \mathrm{~V}$ 97

Figure 4-32 $\mathrm{PRD}_{\mathrm{est}}$ of final estimate after TASI for various triggering modes and an applied SP amplitude of $14 \mathrm{~V}$ 98

Figure 4-33 SNR $_{\text {est }}$ of final estimate after TASI for various triggering modes and an applied SP amplitude of $12 \mathrm{~V}$ 98 


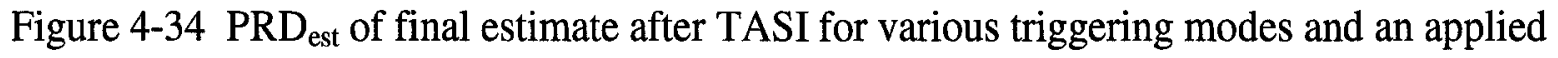
SP amplitude of $12 \mathrm{~V}$ 99

Figure 4-35 SNR $_{\text {est }}$ of final estimate after TASI for various triggering modes and an applied SP amplitude of $10 \mathrm{~V}$ 99

Figure 4-36 $\mathrm{PRD}_{\text {est }}$ of final estimate after TASI for various triggering modes and an applied SP amplitude of $10 \mathrm{~V}$ 100

Figure 4-37 SNR $_{\text {est }}$ of final estimate after TASI for various applied SP amplitudes........... 100

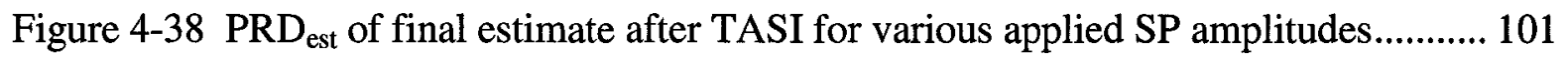

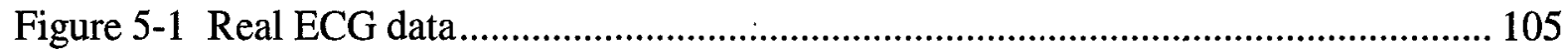

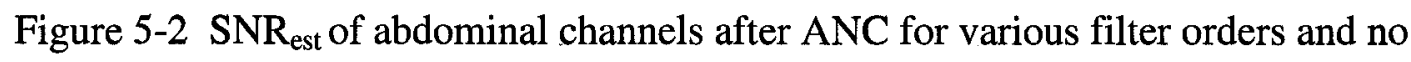
triggering. 107

Figure 5-3 SNR $_{\text {est }}$ of abdominal channels after TANC for various filter orders using triggering with on/off transitions . 108

Figure 5-4 $\mathrm{SNR}_{\text {est }}$ of abdominal channels after TANC for various filter orders using triggering with linear transitions.

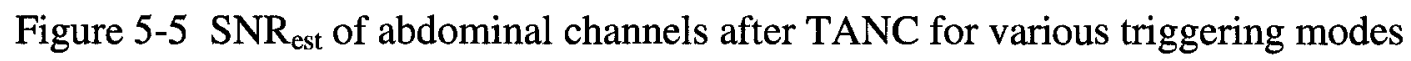
110

Figure 5-6 Estimated FECG after TANC various triggering modes using channel 1 111

Figure 5-7 SNR $_{\text {est }}$ of final FECG estimate after TASI for various filter orders and triggering modes

Figure 5-8 SNR $_{\text {est }}$ of final estimate after TASI for various triggering modes . 113

Figure 5-9 Estimated FECG after TANC and TASI for various triggering modes 114 


\section{List of Tables}

Table 3-1 Synthetic ECG waveform parameters ........................................................ 40

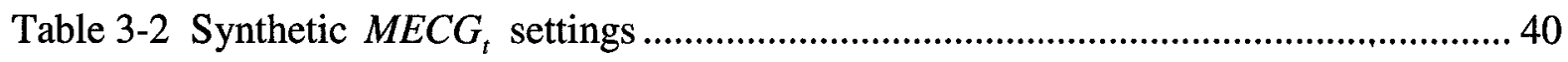

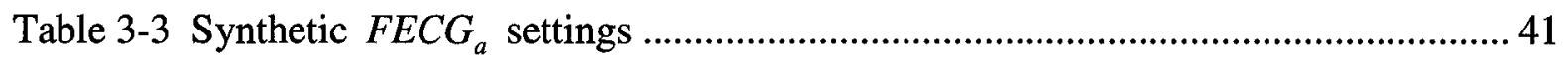




\section{List of Symbols and Abbreviations}

\begin{tabular}{|c|c|}
\hline ANC & Adaptive noise cancellation \\
\hline ASI & Adaptive system identification \\
\hline $\mathrm{Ag}-\mathrm{AgCl}$ & Silver-Silver Chloride \\
\hline AWGN & Additive white Gaussian noise \\
\hline BSS & Blind source separation \\
\hline CECG & Composite electrocardiogram (abdominal) \\
\hline ECG & Electrocardiogram \\
\hline$f_{c}$ & Cutoff frequency \\
\hline FIR & Finite impulse response \\
\hline$f_{s}$ & Sampling frequency \\
\hline FECG & Fetal electrocardiogram \\
\hline $\mathrm{FECG}_{\mathrm{a}}$ & Fetal electrocardiogram measured at maternal abdomen \\
\hline $\mathrm{FECG}_{\text {source }}$ & Fetal electrocardiogram measured at fetal heart \\
\hline $\mathrm{FECG}_{\mathrm{t}}$ & Fetal electrocardiogram measured at maternal thorax \\
\hline FHR & Fetal heart rate \\
\hline FIR & Finite impulse response \\
\hline FPCG & Fetal phonocardiogram \\
\hline HRV & Heart rate variability \\
\hline LMS & Least-mean-square \\
\hline LPF & Low-pass filter \\
\hline$m_{t}$ & Weighted triggering gradient parameter \\
\hline MECG & Maternal electrocardiogram \\
\hline $\mathrm{MECG}_{\mathrm{a}}$ & Abdominal maternal electrocardiogram \\
\hline $\mathrm{MECG}_{\text {source }}$ & Maternal electrocardiogram measured at maternal heart \\
\hline $\mathrm{MECG}_{\mathrm{t}}$ & Thoracic maternal electrocardiogram \\
\hline MES & Myoelectric signal \\
\hline PCG & Phonocardiogram \\
\hline
\end{tabular}


PRD

PRD $_{\text {est }}$

PPRD

RLS

SNR

SNR $_{\text {est }}$

SP

TANC

TASI

$\mu$

$w_{t}$

$\lambda$
Percent residual difference

Estimated percent residual difference

Average pulse percent residual difference

Recursive least-squares

Signal-to-noise ratio

Estimated signal-to-noise ratio

Stimulus pulse

Triggered adaptive noise cancellation

Triggered adaptive system identification

LMS step-size parameter

Trigger weight

RLS forgetting factor 


\section{Chapter 1 \\ Introduction}

\subsection{Overview}

Analysis of the electrocardiogram (ECG) is a simple and noninvasive method of effectively assessing a patient's general health and diagnosing cardiovascular diseases. The ECG can also be used to clinically evaluate the health of prenatal infants. The antepartum fetal ECG (FECG) can be used for the diagnosis of fetal position, congenital heart defects and fetal life $[1,2]$. Variations in the FECG waveform morphology can provide important diagnostic information that is not available in current fetal monitoring techniques, such as ultrasound and phonocardiography. For example, the duration of the QRS complex can be used to distinguish between supraventricular (narrow complex) and ventricular (wide complex) tachycardia (fetal heart rate $\geq 200 \mathrm{bpm}$ ). Also, the position and sign of the $\mathrm{P}$ wave can be used to distinguish between various forms of supraventricular tachycardia [3]. Such evaluations offer the opportunity to combat prenatal health conditions sooner, and offer prenatal awareness of potential physical abnormalities and complications. Invasive methods to acquire the antepartum FECG have associated health risks, such as infection and stress to the fetus, as well as the difficulty of accessing the fetus during this period. Given the sensitivity and inaccessibility of the in utero environment, invasive methods are restrictively performed only during cephalic presentation during labor when there is access to the fetal scalp, which is an accurate measurement location. The FECG can be acquired by inserting an electrode through the dilated cervix and attaching it under the skin of the fetal scalp. 
Prior to childbirth, FECG monitoring is only feasible using noninvasive methods; however, a noninvasive abdominal recording using surface electrodes reveals a composite signal containing an additive mixture of the FECG with unwanted interference. The abdominally recorded FECG has low signal power since the measurement site is away from the source; thus, it is highly susceptible to interference. The dominating interference is the maternal ECG (MECG), which is much stronger than the FECG. Other interfering signals include power-line coupling, muscular and respiratory interference, thermal noise (from electronic equipment), and the electrode-skin interface. Thus, an interesting signal extraction problem is presented.

\subsection{Objective}

The objective of this thesis is to investigate a novel multichannel and multimodal FECG extraction and enhancement technique using linear adaptive filtering with triggering, based on noninvasive abdominal measurements. FECG extraction refers to the separation of the abdominal composite signal into its maternal and fetal components. FECG enhancement, on the other hand, refers to enhancing the extracted fetal component by attenuating other interfering signals (e.g., muscular interference, thermal noise, etc).

Adaptive filtering is capable of extracting the FECG by attempting to model and subtract the maternal component of the abdominal signal; however, due to the fact that the MECG and FECG overlap in both the time and frequency domains, filter adaptation that is meant to attenuate the MECG also partially attenuates the FECG component that occurs simultaneously. This is the basis for the novel inclusion of triggering in the adaptive scheme. 
In this work, we show that by controlling the periods of adaptation in an intelligent manner using triggering, a more accurate FECG signal can be extracted. The concept of triggering is proposed with the inclusion of an extra modality to be combined with the electrical modality of the ECG, to provide temporal information about the FECG. This extra modality could be the fetal heart sound or Doppler ultrasound data, both of which can identify fetal heartbeat occurrences without interference from the maternal heartbeat.

Furthermore, we investigate the extension of triggering to FECG enhancement using multiple abdominal channels. This addresses the low SNR issue associated with noninvasive FECG measurements and enhances the FECG power above that of the remaining noise sources. Adaptive filtering can be applied to reduce uncorrelated noise between multiple channels, and, once again, intelligent triggering can be used to select desired periods of adaptation.

\subsection{Contributions}

1. The novel use of triggered adaptive noise cancellation for FECG extraction has been proposed, tested and verified. This scheme is applied with the intent that an additional modality, such as the fetal phonocardiogram or fetal ultrasound data, can be incorporated to address a limitation of current FECG extraction techniques. In this

regard, the following paper has been published and presented at the $29^{\text {th }}$ Canadian Medical and Biological Engineering Conference in Vancouver, Canada:

V. Badee, A. D. C. Chan and R. M. Dansereau, "Fetal ECG extraction using triggered adaptive noise cancellation", $29^{\text {th }}$ CMBEC, June 2006. 
2. It was shown, through the use of simulated FECG data as well as real data, that the novel use of triggering in a multichannel adaptive system identification scheme is advantageous to enhancing the FECG estimate. Furthermore, the scheme with triggering is realized to be more robust than that without the use of triggering. In this regard, the following abstract has been published and presented at the $16^{\text {th }}$ World Society of Cardio-Thoracic Surgeons Congress in Ottawa, Canada:

V. Badee, A. D. C. Chan and R. M. Dansereau, "Adaptive multichannel fetal ECG extraction and enhancement", $16^{\text {th }}$ WSCTS Congress, August 2006.

3. Triggered adaptive filtering has been extended with weighted transitions to address limitations of the triggering method itself, and the RLS adaptive algorithm has been updated to support the novel use of weighted transitioning. In this regard, the following paper is to be submitted to the $30^{\text {th }}$ Canadian Medical and Biological Engineering Conference in Toronto, Canada:
V. Badee, R. M. Dansereau and A. D. C. Chan, "Fetal ECG extraction and enhancement using triggered adaptive filtering with weighted transitions", $30^{\text {th }} \mathrm{CMBEC}$, to be submitted for publication, June 2007. 


\subsection{Organization}

This thesis is organized as follows:

\section{Chapter 2: Background}

The background relevant to the thesis scope is presented. The problem is formulated, a description of the biological processes involved is given, and a literature review is conducted.

\section{Chapter 3: FECG Extraction using TANC}

This chapter introduces the theory of triggered adaptive noise cancellation (TANC) and applies it to FECG extraction. Simulations are performed using synthetic ECG data and the results are discussed.

\section{Chapter 4: Multichannel FECG Extraction and Enhancement}

The concept of triggering is extended to multiple channels to aid in the enhancement of the desired signal. This is presented using a triggered adaptive system identification (TASI) scheme that follows TANC. The data used to test this system are real data, using a stimulus pulse to represent the FECG.

\section{Chapter 5: Simulation Study with Real Data}

This is a continuation of the triggered extraction and enhancement schemes with the inclusion of multiple channels of real MECG and FECG data.

\section{Chapter 6: Conclusions}

The thesis is concluded with a summary of the results and suggested future research. 


\section{Chapter 2 \\ Background}

\subsection{Problem Formulation}

We would like to identify a noninvasive solution to extract the FECG from an abdominal composite signal. Noninvasive ECG data can be acquired using surface electrodes. Figure 2-1 illustrates the ECG sources and the transformations that occur before they are acquired. Note that additive noises, which may be due to power-line coupling, muscular and respiratory interference, thermal noise (from electronic equipment), and the electrode-skin interface, are not included in this formulation.

$\mathrm{MECG}_{\text {source }}$ is the signal formed by the maternal heart before passing through body tissues. As electrical signals travel through tissue matter, they are distorted. This is called the tissue-filter effect. Thus, as $\mathrm{MECG}_{\text {source }}$ propagates through the chest, it is distorted by an unknown tissue filter, $g_{2}$, before it is acquired at the thorax as $\mathrm{MECG}_{\mathrm{t}}$. The abdominally acquired composite ECG, CECG, contains another variation of $\mathrm{MECG}_{\text {source, }} \mathrm{MECG}_{\mathrm{a}}$, transformed by a different unknown tissue filter, $g_{1}$. The tissue filters $g_{1}$ and $g_{2}$ differ since the signals that are recorded from different measurement sites have propagated through

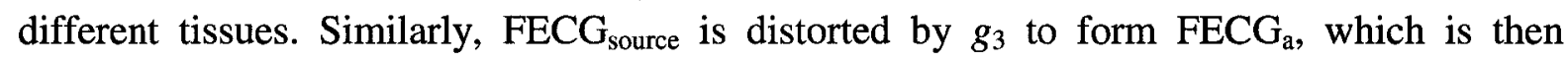
additively combined with $\mathrm{MECG}_{\mathrm{a}}$ to form CECG. CECG contains the desired signal of interest, $\mathrm{FECG}_{\mathrm{a}}$, with unwanted interference from $\mathrm{MECG}_{\mathrm{a}}$ (as well as random noise). Similarly, $\mathrm{MECG}_{\mathrm{t}}$ may contain interference from $\mathrm{FECG}_{\mathrm{t}}$, i.e., a distorted $\mathrm{FECG}_{\text {source }}$ that has 
propagated to the maternal chest; however, this can be ignored due to the negligible signal strength of $\mathrm{FECG}_{\mathrm{t}}$, which does little to distort the acquired thoracic signal.

To formulate the problem with a noninvasive approach, we set our goals on extracting $\mathrm{FECG}_{\mathrm{a}}$, instead of $\mathrm{FECG}_{\text {source, }}$, as the optimal solution. Thus, $g_{2}$ and $g_{3}$ will be ignored and a simpler problem formulation is illustrated in Figure 2-2. The only tissue filter that is considered now is $h_{1}$, denoting the distortion imposed on $\mathrm{MECG}_{\mathrm{t}}$ to form $\mathrm{MECG}_{\mathrm{a}}$. In relation to Figure 2-1, $h_{1}$ can be defined as

$$
h_{1}(t) \equiv g_{2}^{-1}(t) * g_{1}(t)
$$

Using the simplified formulation of Figure 2-2, an estimate of the FECG can then be extracted from the abdominal composite signal using Equation 2.2. In this equation, the first term, $C E C G(t)$, cannot be directly separated into its components, $M E C G_{a}(t)$ and $F E C G_{a}(t)$, since it is noninvasively observable only as a composite signal. In order to extract the $F E C G_{a}(t)$ component, one can subtract an estimate of the $M E C G_{a}(t)$ component, as shown in Equation 2.2. To estimate $M E C G_{a}(t)$, it can be replaced with the physically measurable thoracic MECG, $M E C G_{t}(t)$, passed through $\hat{h}_{1}$, an estimate of the tissue filter, $h_{1}$. This is represented in Equation 2.3. 


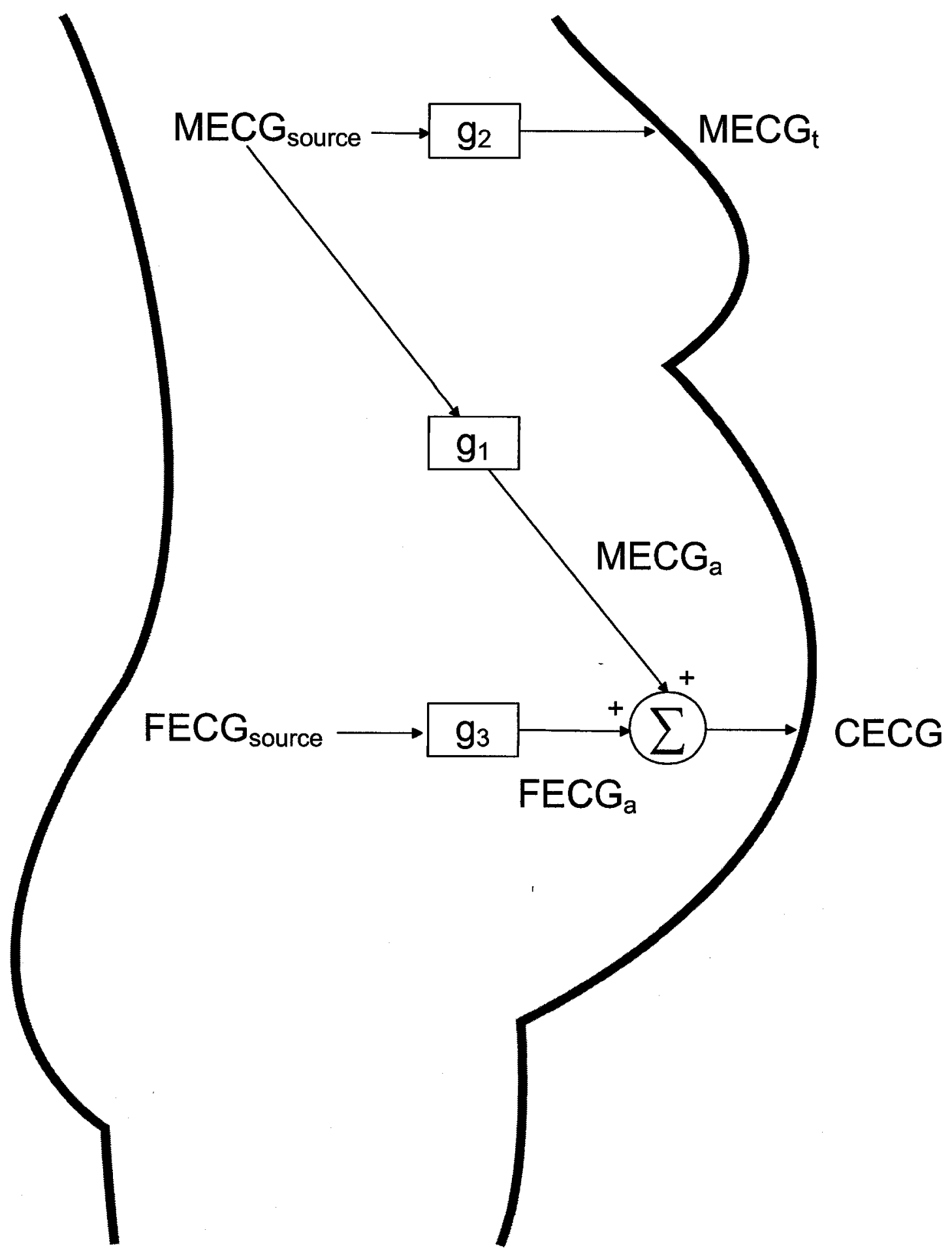

Figure 2-1 Problem formulation

8 


$$
\begin{aligned}
& F E \hat{C} G_{a}(t)=(C E C G(t))-M \hat{E C} G_{a}(t) \\
& =(C E C G(t))-\left(M E C G_{t}(t) * \hat{h}_{1}(t)\right)
\end{aligned}
$$

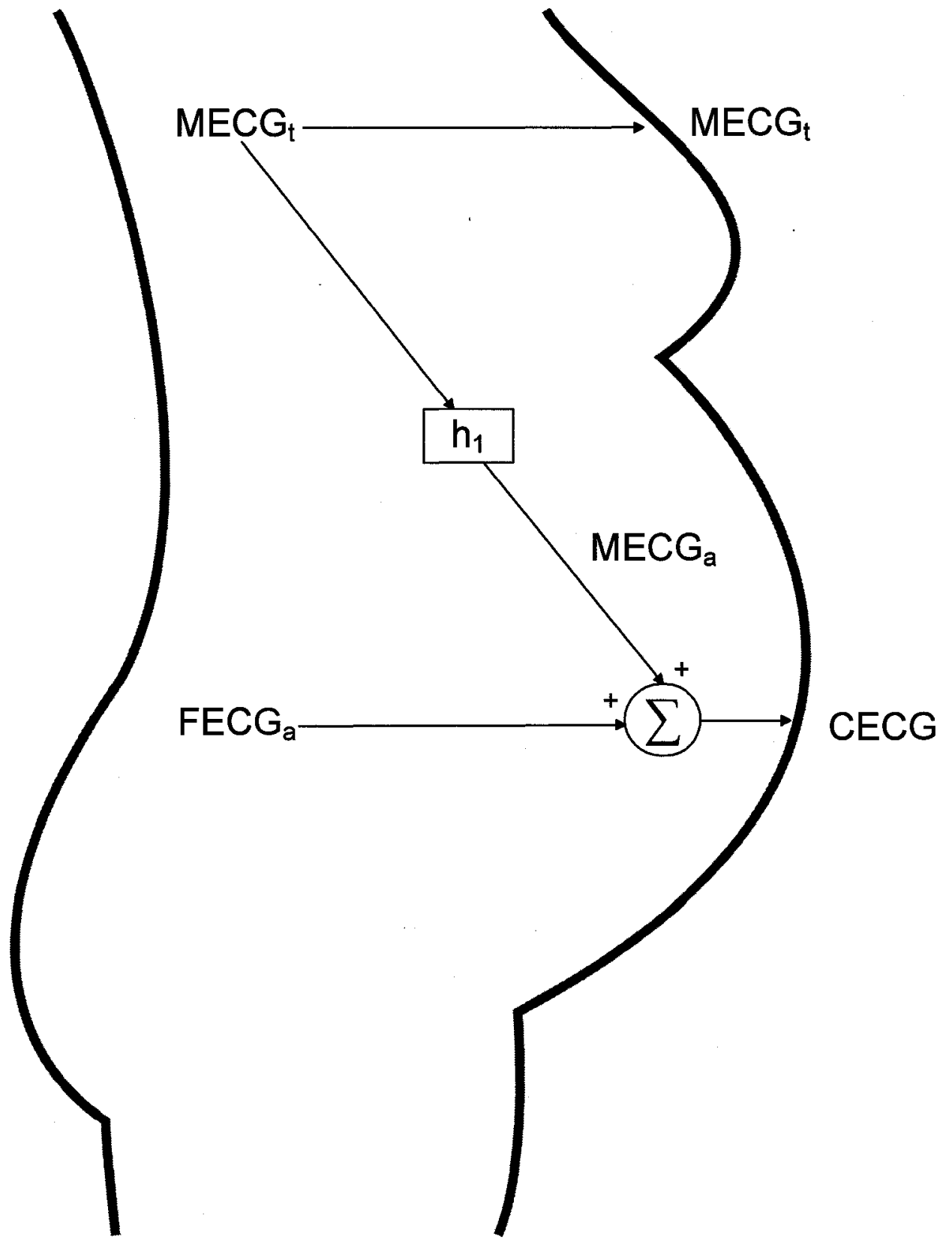

Figure 2-2 Simplified problem formulation 


\subsection{Fetal Monitoring Techniques}

A number of different techniques are currently used to monitor the fetal state. These include the monitoring of the intrapartum fetal ECG during delivery as well as antepartum monitoring of the fetus using its heart sounds or phonocardiogram (PCG). Ultrasound is also currently used to image the fetus and monitor the heart rate. Many other monitoring techniques exist, but are beyond the scope of this work and will not be presented. Some examples of these are magnetocardiography (to monitor fetal development and status), electroencephalography (to monitor fetal brain function), fetal scalp blood sampling (to monitor fetal $\mathrm{pH}$ levels) and the fetal photoelectric plethysmographic pulse (to monitor fetal peripheral pulse and oxygen saturation levels).

It is important to note that the figures in this section represent the postnatal anatomy of the heart. The antenatal fetal heart contains two shunts to account for receiving oxygenated blood from the mother, via the placenta, instead of the lungs. The first is an opening between the atria, called the foramen ovale, which allows blood to enter the left atrium from the right atrium. In most individuals, this opening closes after birth to form the fossa ovalis. The second is a channel between the pulmonary artery and the aorta, called the ductus arteriosus, which allows blood received from the placenta to be distributed to the fetal body, thus bypassing the lungs. This shunt also closes after birth, separating the pulmonary and systemic blood circulation systems. These shunts in the fetal heart do not considerably affect the important features of fetal signals, such as the ECG and PCG. 


\subsubsection{Intrapartum ECG}

The most accurate method of realizing the fetal state is through the intrapartum ECG. These data can be collected using rectal, vaginal and cervical probes; however, the best results are obtained using an intrauterine electrode that is attached to the fetal scalp [1]. Although highly accurate, the in utero FECG is normally restricted to measurement during childbirth, when access to the fetal scalp is easiest; however, due to sensitivity of the in utero environment and the developing fetus, intrapartum FECG measurement may cause fetal scalp damage and neonatal jaundice [4].

The FECG waveform can provide vital information about the fetal state. Conditions such as hypoxia (reduced oxygen flow), hypotension (low blood pressure), anemia (deficiency in red blood cells), arrhythmias (irregular heart beats) and autonomic nervous system conditions can be diagnosed. One can refer to [5] for a more detailed review of these. The FECG waveform is similar to the adult ECG (seen in Figure 2-3) [6].

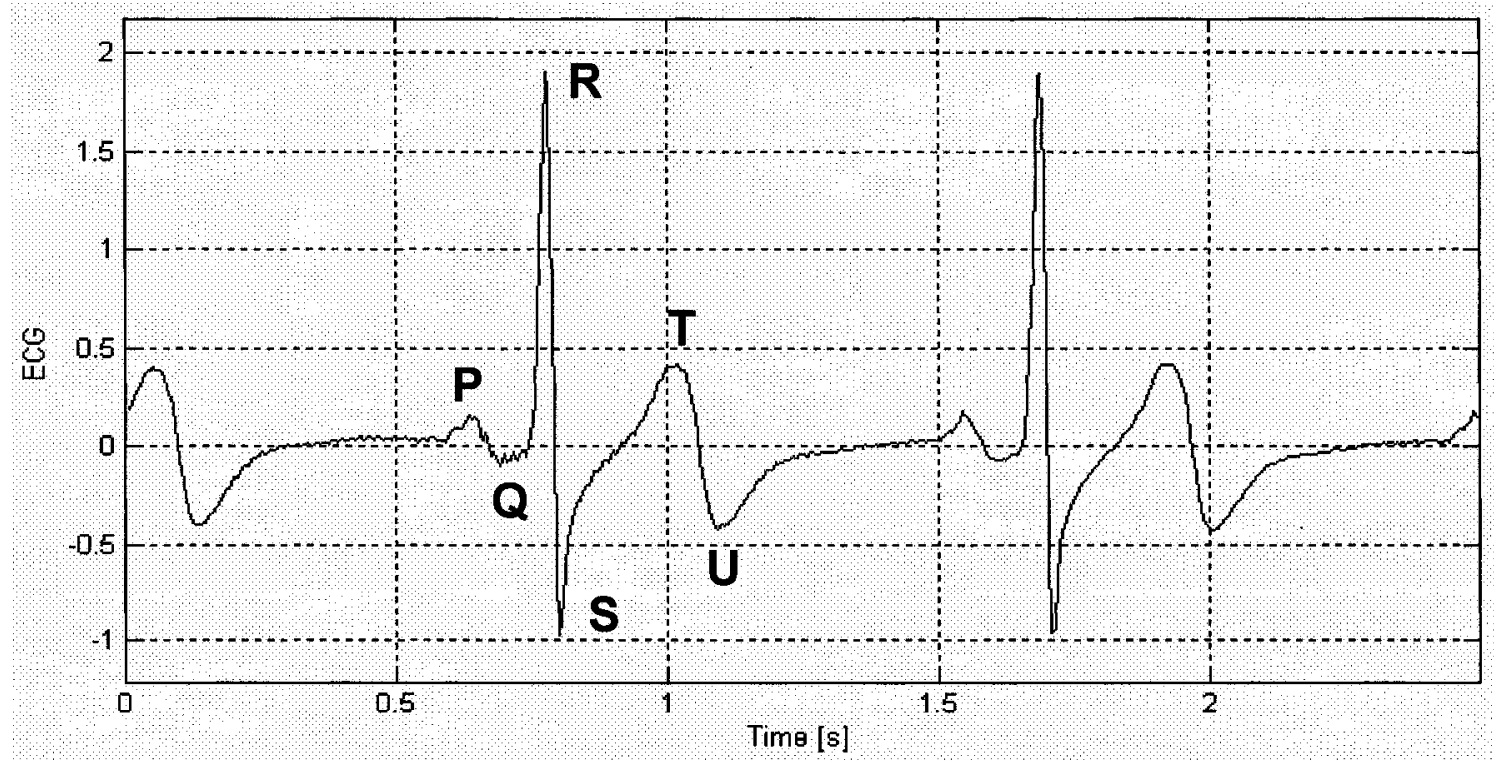

Figure 2-3 Normal electrocardiogram 


\subsubsection{Origin of the Electrocardiogram}

The origin of the ECG arises from electrical events generated by cardiac cells, causing the contraction and relaxation of myocardial cells. The waveform shown in Figure 23 represents a series of depolarizations/repolarizations that occur as a result of ionic flow across cardiac cell membranes in specialized chambers (shown in Figure 2-4). This ionic activity is associated with the cardiac muscles contracting or relaxing, and manifests as a measurable electrical potential.

The pumping action that forces blood out to the body and lungs is provided by the ventricles. This ventricular contractile phase is called the systole. The atria are responsible for storing blood during this time. After ventricular contraction, the atria contract to force blood into the now relaxing ventricles. This ventricular relaxation phase is called the diastole.

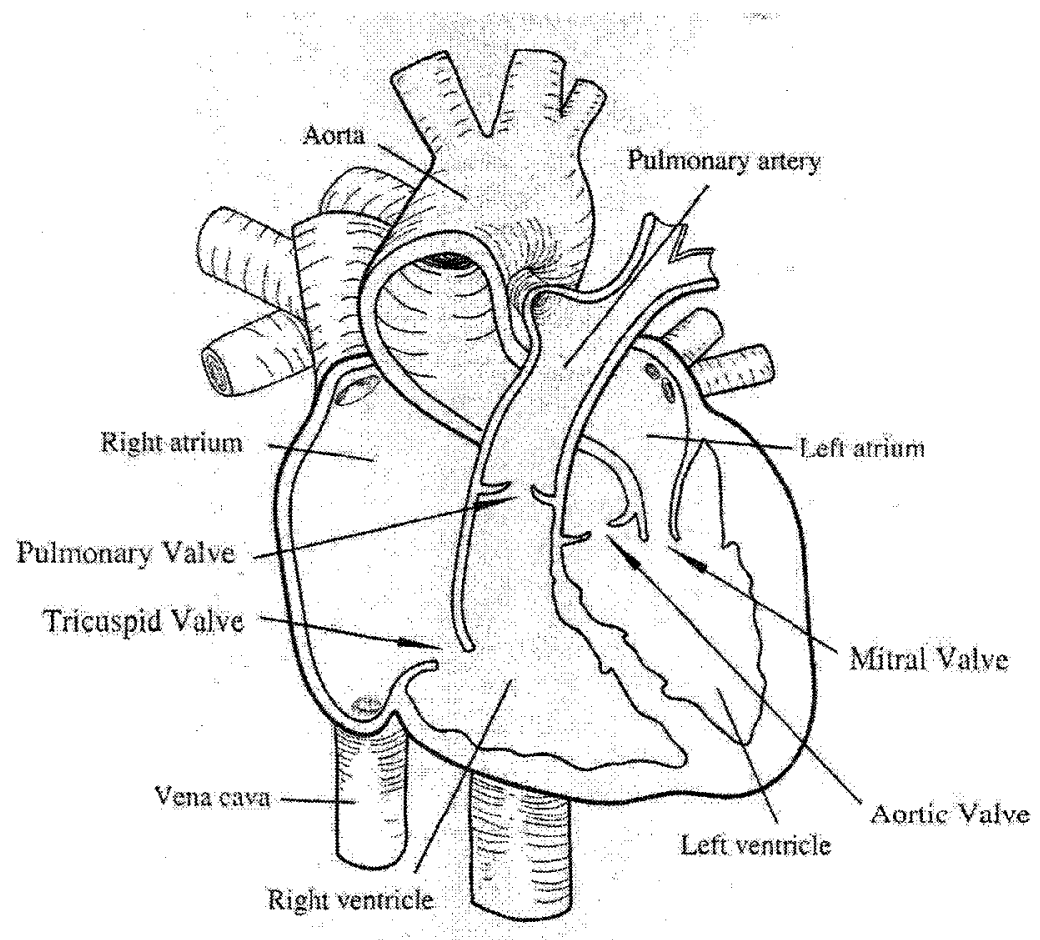

Figure 2-4 Heart anatomy, image reproduced from [7] 
The $P$ wave corresponds to atrial contraction or depolarization, i.e. electrical potential formed as the high concentration of sodium $\left(\mathrm{Na}^{+}\right)$and calcium $\left(\mathrm{Ca}^{+}\right)$cations present outside the cell membrane enter within. The $Q R S$ complex, the most prominent feature of the waveform, corresponds to the contraction of the larger and more muscular ventricles. The $T$ wave follows as the ventricles relax or repolarize, i.e. the cells return to resting state through the efflux of potassium $\left(\mathrm{K}^{+}\right)$cations out of the cardiac cells. The $U$ wave, which is not always seen in ECG data acquired using clinical lead configurations, is believed to correspond to the relaxation of the Purkinje fibers (or Papillary muscles), which are responsible for conducting impulses from the atrioventricular node to the ventricular myocardium to initiate the next systole.

To ensure that cardiac muscles relax before a contraction, they have an absolute refractory period of about $250 \mathrm{~ms}$, about as long as the contraction itself, which begins at the end of a contraction [7].

\subsubsection{Phonocardiogram}

Auscultation techniques for diagnosis of heart conditions can often be quite subjective and dependent on the experience of the physician. This qualitative measure may be insufficient to diagnose some heart diseases, and indeed it has been shown that the human ear is poorly suited for cardiac auscultation [8]. The PCG provides a quantitative measure of heart sounds, exposing many features through digital signal processing, such as timing, frequency content, location in cardiac cycle and envelope shape. A normal PCG, as shown in Figure 2-5, typically has two main heart sounds S1 and S2. The origin of these is detailed in Section 2.2.2.1. 
Another advantage of acquiring PCGs over auscultation is the ability to permanently store the acquired data for post-processing (especially vital due to the PCG's transient and highly non-stationary nature), second opinions and future reference. This information is used to monitor the fetal heart rate (FHR). Fetal distress may be indicated by tachycardia (FHR > $160 \mathrm{bpm}$ ), bradycardia (FHR $<100 \mathrm{bpm}$ ), as well as an irregular FHR [1].

The PCG is acquired using a digital stethoscope with the standard sensor being a microphone. It has a frequency range between $5-2000 \mathrm{~Hz}$ [9]; however fetal PCGs can be acquired successfully at frequencies between $75-105 \mathrm{~Hz}$ [1].

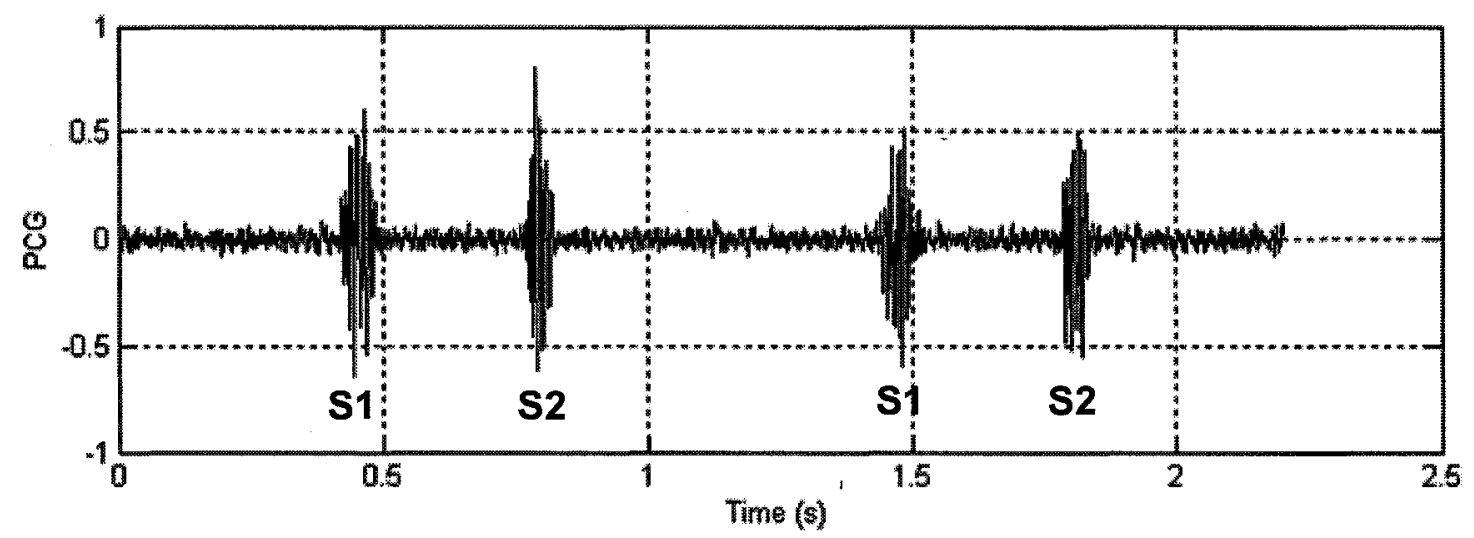

Figure 2-5 Normal phonocardiogram

\subsubsection{Origin of the Phonocardiogram}

The origin of the PCG arises mainly from the acceleration and deceleration of blood, causing the sharp closing of valves. A heartbeat consists of two main sounds, namely S1 and S2. S1 approximately marks the beginning of the systole, or the contraction of heart chambers, while S2 approximately marks the end of the systole. These heart sounds are similar in both the antenatal and prenatal PCG. The processes explained in this section 
describe the flow of blood through the antenatal heart (recall the shunts described in Section 2.2 that allow the prenatal heart to bypass blood flow to the fetal lungs); however, the processes that account for the origin of the S1 and S2 heart sounds remain the same.

Figure 2-6 shows the processes that contribute to the formation of heart sounds. In Figure 2-6(a), oxygen-rich blood enters the left atrium via the pulmonary veins (from the lungs), and oxygen-depleted blood enters the right atrium via the superior and inferior vena cava (from the body). This is the relaxation of the atria. In Figure 2-6(b), the blood is allowed to enter the ventricles as the tricuspid and mitral valves open. Figure 2-6(c) marks the origin of the S1 sound and the approximate start of the ventricular systole. This is formed as the tricuspid and mitral valves snap shut to prevent the backflow of blood into the atria. Blood is now trapped in the ventricles and reverses flow, adding an additional low-frequency vibration. In Figure 2-6(d), the pulmonic and aortic valves open to allow blood out of the heart. Blood accelerates into the arteries as the pressure is released. Figure 2-6(e) shows the path of blood as it leaves the heart. Oxygen-depleted blood in the right ventricle exits via the pulmonary arteries leading to the lungs to be replenished. Oxygen-rich blood in the left ventricle exits via the aorta leading to the rest of the body. Figure 2-6(f) marks the origin of the S2 sound. This is formed as the pulmonic and aortic valves snap shut, and blood in the aorta and pulmonary arteries decelerates and changes direction of flow. This marks the end of the ventricular systole. 
(a)

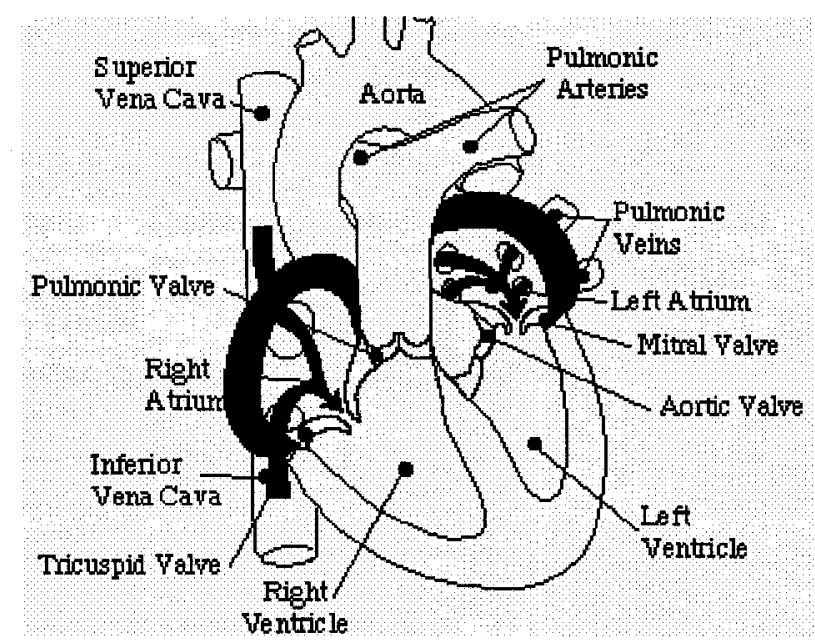

(b)

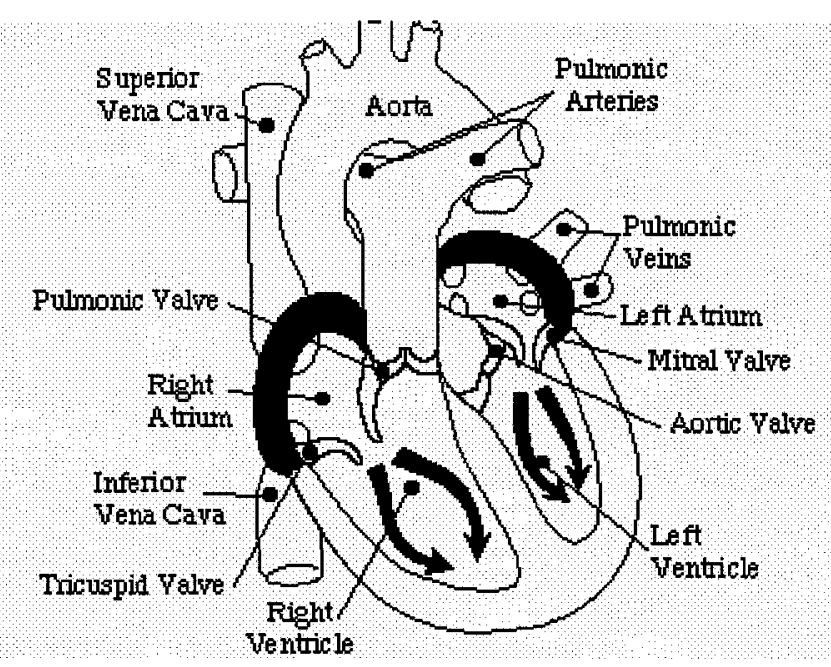

(c)

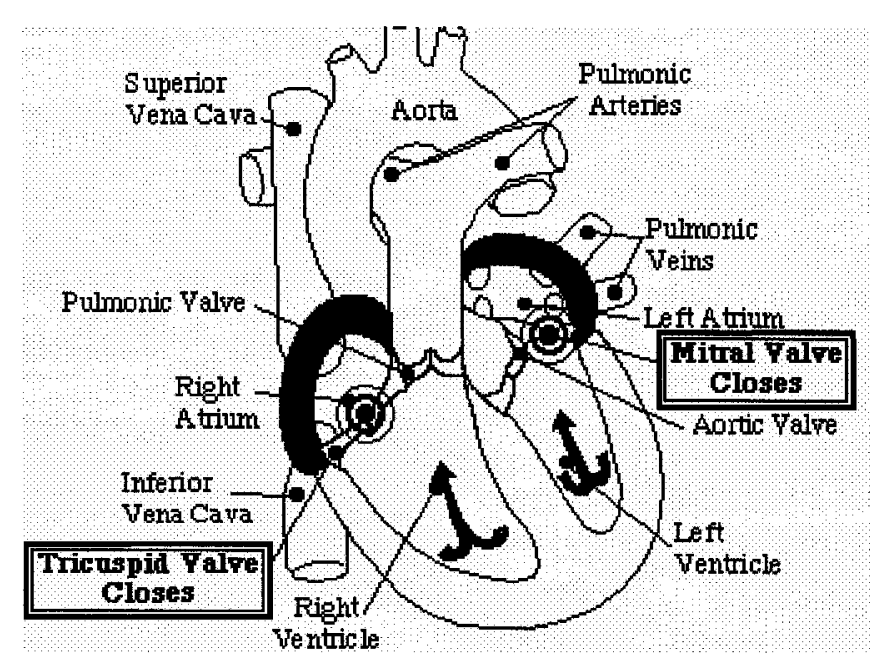


(d)

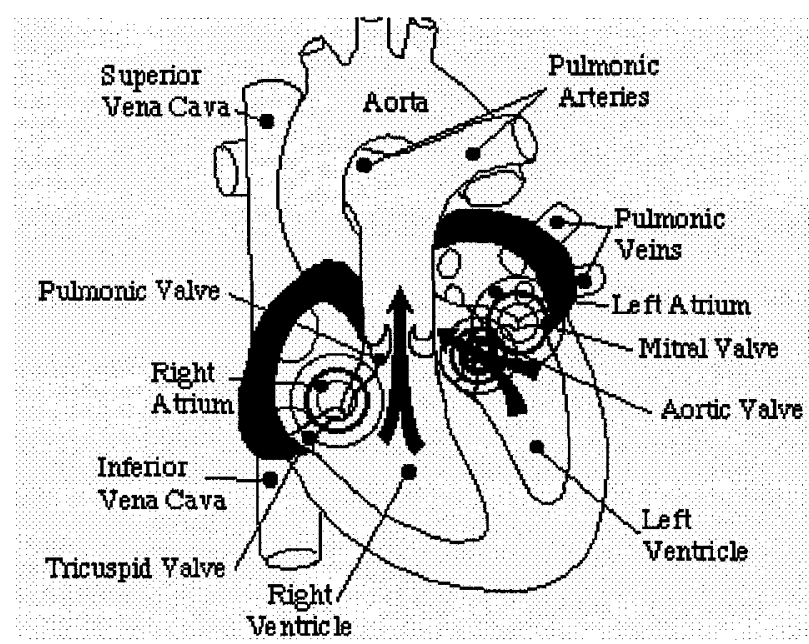

(e)

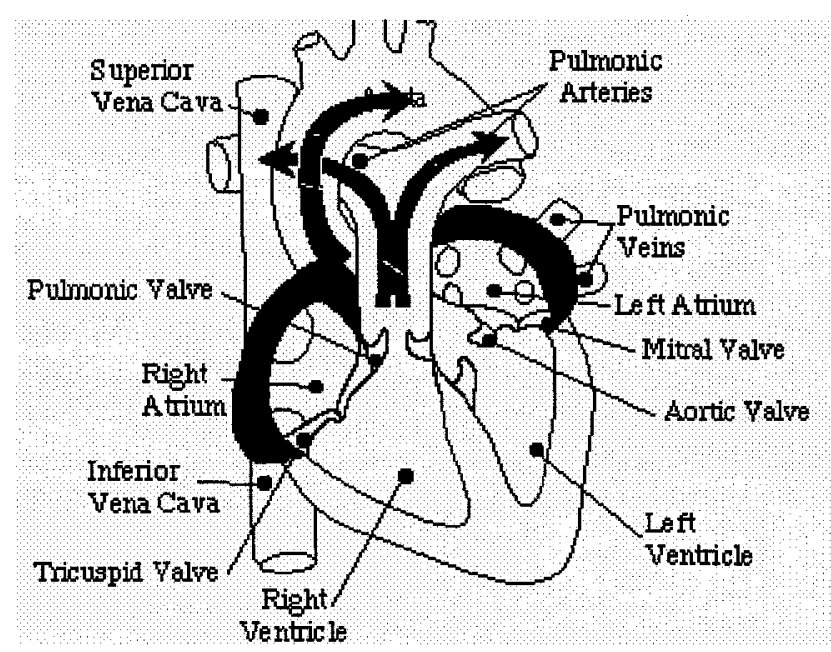

(f)

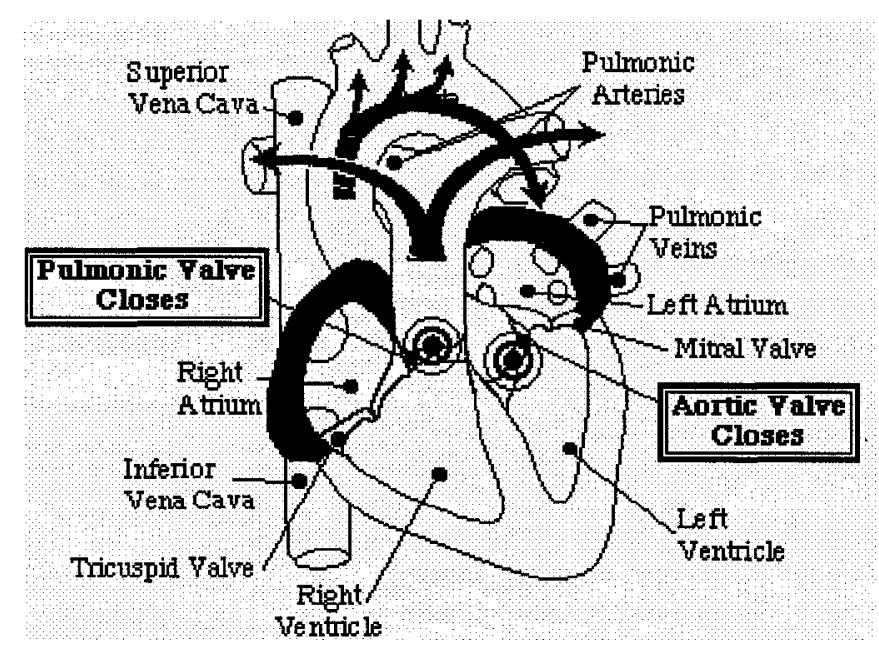

Figure 2-6 Origin of heart sounds, images reproduced from [10] 
The S1 and S2 heart sounds are each formed from the near simultaneous closing of two separate heart valves, and in this sense are considered discrete sounds; however, the closure of the two separate valves is not exactly simultaneous. The order of the closures can be intuitively explained. The pressure during a systole is much greater in the left ventricle than the right (the left ventricle sends blood out to the rest of the body). Thus, the mitral valve closes slightly before the tricuspid valve in S1. Similarly, during the diastole, blood leaving the heart through the aorta is at a higher pressure than blood leaving through the pulmonic arteries. Thus, the aortic valve closes slightly before the pulmonic valve in $\mathrm{S} 2$.

Two other heart sounds exist as well, S3 and S4. S3, a low-amplitude, low-frequency vibration, occurs due to the vibration of the ventricular muscle walls after the rapid filling phase of the ventricles. This sound is sometimes audible. S4, also called the atrial heart sound, occurs due to the contraction of the atria to force blood into the ventricles. This sound is inaudible. Figure 2-5 shows a normal PCG. The first and third pulses represent $\mathrm{S} 1$, and the second and fourth represent S2. S3 and S4 are not visible in this figure and indeed are not necessary for the calculation of the FHR.

\subsubsection{Ultrasound}

Ultrasound is a very popular noninvasive medical technique used in various specialties, including obstetrics, gynecology and cardiology. Its high acceptance is due to its comparatively low cost, high speed, small size and the fact that it does not use radiation. The disadvantage of this technique is the low-resolution images it produces, due to speckle "noise", and the temperature increases it causes in tissues making it unsafe for long-term monitoring [11]. The use of Doppler ultrasound is a common means of detecting fetal 
heartbeats in order to calculate the FHR. Doppler ultrasound provides a functional image, as opposed to the anatomical image provided by regular pulse-echo ultrasound.

The theory behind Doppler ultrasound was proposed by Christian Doppler in 1842 . The Doppler Effect states that the frequency of a wave emitted by or reflected from a moving object is dependent on the velocity of the object. By emitting sound waves into the fetal cardiovascular system and measuring the frequency of the reflected wave, Doppler ultrasound can be used to measure blood flow velocities. Instantaneous fetal heart rates can then be found using threshold detection techniques $[11,12]$. For a more detailed discussion of the theory behind Doppler ultrasound, refer to [13].

Although Doppler ultrasound is currently the most common noninvasive method of measuring the FHR, it does contain limitations [14]. Due to the sensitivity of focusing the beam directly onto the fetal cardiovascular system, there is the chance of picking up other fetal or maternal organs instead. Incorrect measurements may also result from fetal movement.

\subsection{Fetal ECG Extraction using Adaptive Noise Cancellation}

In Equation 2.2, it was shown that in order to estimate the FECG noninvasively, an estimate of the tissue filter, $h_{1}$, is required. One possible technique that can be used to estimate $h_{1}$ is using adaptive filters. The classic ANC approach to fetal ECG extraction was introduced by Widrow et al. in 1975 [15]. The ANC scheme is shown in Figure 2-7 as applied to fetal ECG extraction. 


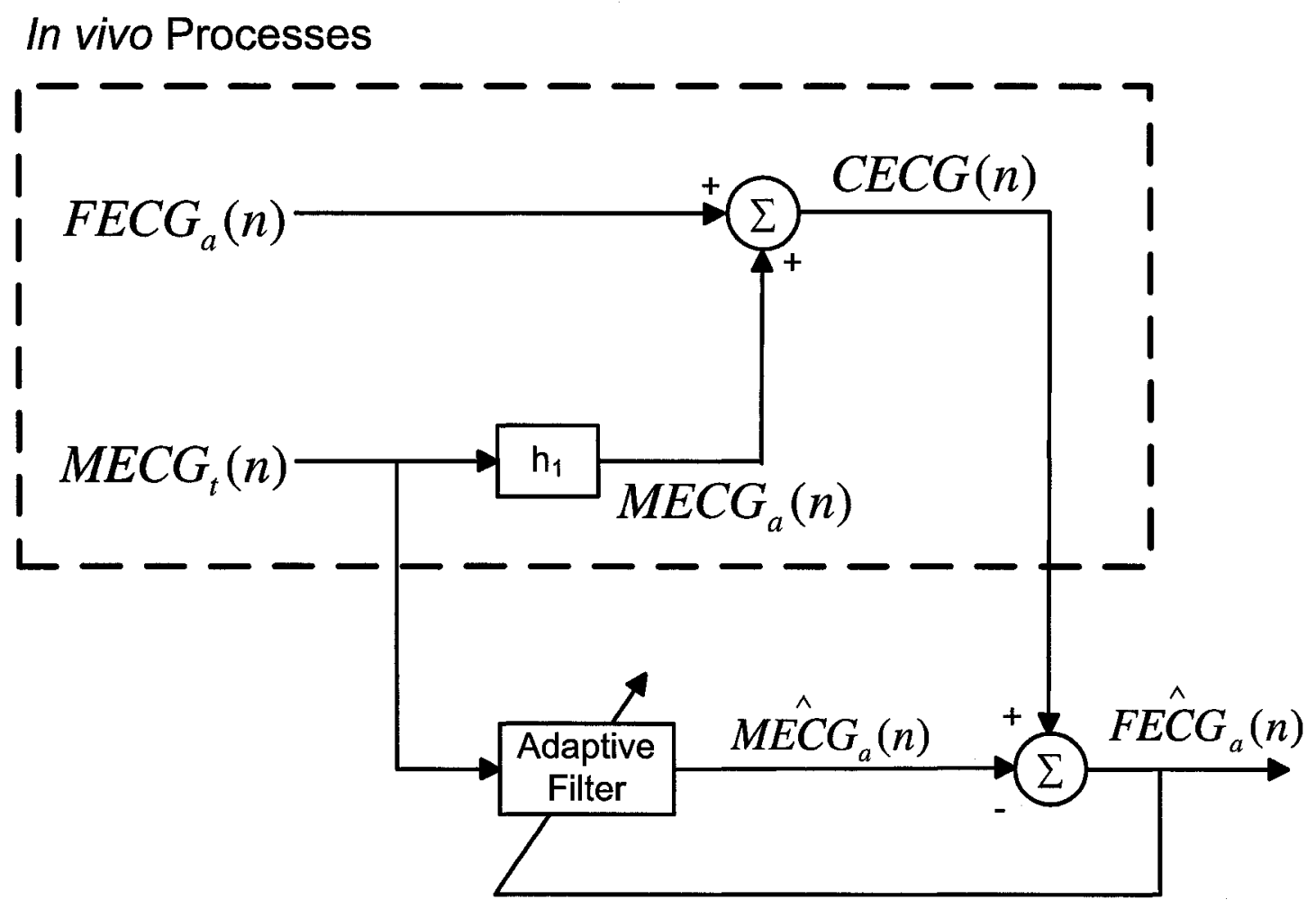

Figure 2-7 Adaptive noise cancellation scheme applied to FECG extraction

The measurable signals consist of the thoracic MECG, $M E C G_{t}$, and the abdominal composite ECG, CECG. These are acquired through noninvasive surface electrodes placed on the chest and abdomen, respectively, of the subject. The correlation between $M E C G_{t}$ and $M E C G_{a}$ is exploited by the adaptive filter to estimate the body's tissue filter, $h_{1}$. This is based on three reasonable assumptions:

1. The MECG and FECG are independent signals. This assumption is based on the fact that the maternal and fetal heartbeats have physically independent sources. 
2. $M E C G_{t}$ and $M E C G_{a}$ are correlated. This assumption is based on the fact that the maternal heartbeat originates from one source.

3. $M E C G_{t}$ is assumed to be free of any FECG components. This assumption is dependent on the relatively low amplitude of the fetal cardiac vector. Given the distance between the FECG source and the MECG measurement site, attenuation caused by the tissue filter will have rendered the effect of $F E C G_{a}$ on $M E C G_{t}$ negligible.

The classic adaptive filter contains an FIR transversal filter whose taps are updated according to an adaptive algorithm. The use of an adaptive filter, as opposed to a fixedweight filter, is necessary since the characteristics of the channel through which the ECG propagates are unknown and vary both in time and from person to person. A properly designed adaptive filter can provide a reasonable estimate $M E C G_{a}$, which is subtracted from $C E C G$ to extract $F E C G_{a}$.

Proper design of this filter includes the selection of an effective adaptation algorithm that minimizes the error signal. In early literature, the least mean square (LMS) adaptation algorithm was most commonly adopted, and indeed so in Widrow's own works. The recursive least-squares (RLS) algorithm was subsequently introduced, and is currently commonly used. A description of the LMS and RLS algorithms is provided in Sections 2.3.1 and 2.3.2, respectively.

Referring back to [15], the experiments carried out showed modest results and paved the way for further research in this area. A multireferenced (i.e., multiple thoracic leads) 
adaptive noise canceller demonstrated the applicability of ANC in FECG extraction; however, the resulting estimate was still corrupted by background muscle activity. In the early 1980's, this problem was addressed by Ferrara and Widrow through the proposition of a time-sequenced adaptive filter [16-18]. The premise of this filter is to perform adaptive filtering on nonstationary signals that contain statistically recurring properties. This applies nicely to the ECG, which satisfies these criteria. In [18] the time-sequenced adaptive filter attempts to adapt to the desired signal separately from the interfering one in a multichannel enhancement scheme. The scheme uses two separate adaptive filters; one is enabled only during the desired pulse and the other is enabled in between pulses. Thus, one set of weights is adapted during the FECG and another during background muscle noise. Obviously, $a$ priori knowledge of the temporal location of these pulses is required, and thus this enhancement procedure is performed after an initial noise cancellation phase so that the FECG pulses can be found using peak detection.

\subsubsection{LMS Adaptation}

The least-mean-square (LMS) algorithm, originally developed in [19], is one of the standard techniques of adaptive filtering [20]. It is a stochastic gradient algorithm, updating weights after each iteration based on an approximation of the instantaneous gradient, as opposed to the method of steepest descent (deterministic gradient algorithm). This property allows for minimal computational complexity, however it does introduce gradient noise. The simplicity and robustness of the LMS algorithm have made it a standard adaptive algorithm to which the performances of other algorithms are compared.

Initially, the FIR filter output is computed as 


$$
y(n)=\vec{x}^{H}(n) \vec{w}(n),
$$

where $y(n)$ is the $N^{\text {th }}$ order filter output at sample $n, \vec{w}(n)$ is the filter weight vector and $\vec{x}^{H}(n)$ is the transposed input vector at sample $n$. The error is then calculated as

$$
e(n)=d(n)-y(n),
$$

where $e(n)$ is the estimation error and $d(n)$ is the desired signal at sample $n$. Adaptation is then based on minimizing the error in the mean-square sense, so that the update to the filter tap-weights is computed by

$$
\vec{w}(n+1)=\vec{w}(n)+\mu e^{*}(n) \vec{x}(n),
$$

where $\mu$ is the adaptation step-size parameter (constrained to ensure stability). Ideally, $x(n)$ (i.e., the thoracic MECG) and $d(n)$ (i.e., the abdominal composite ECG) are jointly stationary processes, allowing for an average convergence to the Wiener solution; however, this is not the case since ECG data are nonstationary. The LMS algorithm does its best to attenuate statistically correlated data between $x(n)$ and $d(n)$, and thus the statistically independent data (i.e., the FECG) can be extracted.

When a system's physical model and its variability with time are not well defined, the optimum value for $\mu$ can be computed adaptively. Since $\mu$ is now, in itself, an adaptive process, Equation 2.6 is rewritten as

$$
\vec{w}(n+1)=\bar{w}(n)+\mu(n) e^{*}(n) \bar{x}(n),
$$

where $\mu(n)$ is the adaptive step-size. This is updated after each iteration by

$$
\mu(n+1)=\left[\mu(n)+\alpha \operatorname{Re}\left[\hat{\bar{\Psi}}^{H}(n) e^{*}(n) \vec{x}(n)\right]\right]_{\mu_{-}}^{\mu_{+}}
$$


where $\alpha$ is a small, positive learning-rate parameter, and $\mu_{+}$and $\mu_{-}$represent the upper and lower limits of $\mu$, respectively. $\hat{\bar{\Psi}}$, which denotes an estimate of the gradient of the weight vector, $\vec{w}(n)$, with respect to the step-size, $\mu$, is also computed iteratively using

$$
\hat{\bar{\Psi}}(n+1)=\hat{\bar{\Psi}}(n)+e^{*}(n) \vec{x}(n)-\mu(n) \vec{x}(n) \vec{x}^{H}(n) \hat{\bar{\Psi}}(n) .
$$

A detailed description of adaptive gain and the derivation of Equations 2.7-2.9 can be found in [20].

\subsubsection{RLS Adaptation}

The RLS algorithm does not attempt to minimize statistical assumptions, as is the case with the LMS algorithm [20]. It is a deterministic gradient algorithm, updating filter parameters using all observations from time $t=0$ to present. Since it does not use a stochastic gradient calculation, the RLS algorithm does not suffer as much from gradient noise and can converge in fewer iterations. To allow for continuous operation, and thus actual implementation, the solution can be computed in recursive form.

The output of the FIR filter is computed as

$$
y(n)=\vec{x}^{H}(n) \vec{w}(n-1) .
$$

Since the filter output now incorporates previous samples into the calculation, an a priori estimation error, $\xi$, is used to find the error. This is generally different from the a posteriori estimation error, $e$, used in the LMS algorithm in Equation 2.5. The error output of the RLS algorithm is thus computed using

$$
\xi(n)=d(n)-y(n) .
$$


Recursion in the RLS algorithm is associated with a gain vector, $k$, and the inverse correlation matrix, $P$. These are given by,

$$
\vec{k}(n)=\frac{\lambda^{-1} \vec{P}(n-1) \vec{x}(n)}{1+\lambda^{-1} \vec{x}^{H}(n) \vec{P}(n-1) \vec{x}(n)},
$$

and

$$
\vec{P}(n)=\lambda^{-1} \vec{P}(n-1)-\lambda^{-1} \vec{k}(n) \vec{x}^{H}(n) \vec{P}(n-1),
$$

respectively, where $\lambda$ is a positive constant representing the adaptation forgetting factor, i.e., a measure of the memory of the algorithm. Generally, $\lambda$ is chosen to be close to, but less than, 1. This indicates that the algorithm maintains the use of previous samples in its calculations, thus having more memory. Adaptation is then based on minimizing the error in the least-squares sense. Making use of the recursive form of $k$, the filter weight update is calculated by

$$
\vec{w}(n)=\vec{w}(n-1)+\vec{k}(n) \xi^{*}(n) .
$$

Similar to the selection of an optimum $\mu$ using adaptive gain in the LMS algorithm, it is desirable to incorporate an adaptive memory into the RLS algorithm to calculate an optimum $\lambda$. Equations 2.12 and 2.13 must be updated to include a time-varying $\lambda$, and can be written as

$$
\vec{k}(n)=\frac{\lambda^{-1}(n-1) \vec{P}(n-1) \vec{x}(n)}{1+\lambda^{-1}(n-1) \vec{x}^{H}(n) \vec{P}(n-1) \vec{x}(n)},
$$

and

$$
\vec{P}(n)=\lambda^{-1}(n-1) \vec{P}(n-1)-\lambda^{-1}(n-1) \vec{k}(n) \vec{x}^{H}(n) \vec{P}(n-1),
$$

respectively. $\hat{\bar{\Psi}}$, which was introduced in Equation 2.9 for the LMS algorithm, is calculated for the RLS algorithm using 


$$
\hat{\bar{\Psi}}(n)=\hat{\bar{\Psi}}(n-1)+\vec{S}(n) \vec{x}(n) \xi^{*}(n)-\vec{k}(n) \vec{x}^{H}(n) \hat{\bar{\Psi}}(n-1),
$$

where $\vec{S}$ represents the derivative of the inverse correlation matrix, $\vec{P}$, with respect to $\lambda$, and can be calculated using

$$
\begin{aligned}
& \left.\vec{S}(n)=\lambda^{-1}(n)\left|\vec{I}-\vec{k}(n) \vec{x}^{H}(n)\right| \vec{S}(n-1) \mid \vec{I}-\vec{x}(n) \vec{k}^{H}(n)\right] \\
& +\lambda^{-1}(n) \vec{k}(n) \vec{k}^{H}(n)-\lambda^{-1}(n) \vec{P}(n) .
\end{aligned}
$$

Finally, $\lambda$ is updated after each iteration:

$$
\lambda(n)=\left[\lambda(n-1)+\alpha \operatorname{Re}\left[\hat{\vec{\Psi}}^{H}(n-1) \vec{x}(n) \xi^{*}(n)\right]\right]_{\lambda}^{\lambda_{+}},
$$

where $\alpha$ is a small, positive learning-rate parameter, and $\lambda_{+}$and $\lambda_{-}$represent the upper and lower limits of $\lambda$, respectively. A detailed description of adaptive memory and the derivation of Equations 2.15-2.19 can be found in [20].

\subsection{Fetal ECG Extraction using Blind Source Separation}

More recent literature addresses FECG extraction as a blind source separation (BSS) problem [21-23]. The foundation of this technique is based on the dipole model of the heart's electrical activity [24]. This model assumes that the current source (i.e., the heart) is fixed in position, and the dipole vector, representing the current flow, varies in magnitude and direction over time. This is described by a linear combination of the $x, y$, and $z$ components in an orthogonal lead system. It is shown in [24] that the current source can be modeled by a first-order approximation, thus surface potential measurements can be assumed to be instantaneous linear mixtures of the signals generated by the bioelectric sources. In this way, the signal sources, which are internal and cannot be measured noninvasively, can be 
separated from the measured signal through a mixing matrix. An additive noise component is included in this model to account for noise sources, such as myoelectric interference, thermal noise and power-line coupling. This leads to the relationship

$$
\bar{y}(k)=\vec{M} \bar{x}(k)+\bar{n}(k),
$$

where $\vec{n}(k)$ represents additive noise in sampled time unit $k, \vec{x}$ represents the signal source vector, $\vec{M}$ is the mixing matrix, and $\vec{y}$ is the measurement signal vector (as observed by surface electrodes). The mixing matrix $\vec{M}$ defines the transformation of the bioelectric signal source vector as it propagates through the body and is observed at the surface. This is determined by the geometry of the body, position of the bioelectric sources, placement of the electrodes and the body's tissue-filtering effect [23]. This model corresponds to the BSS model of instantaneous linear mixtures [25].

The use of BSS, based on higher-order statistics, as opposed to classic ANC, has shown promise in current research experiments. A direct comparison between the two methods is presented in [22] with the conclusion that BSS has superior performance at the expense of computational complexity; however, similar to classic ANC, the underlying issue remains that the estimation of the mixing matrix $\vec{M}$ for BSS, or the tissue filter $h_{l}$ for ANC (see Section 2.3), is not accurate enough and prevents both techniques from achieving a performance worthy of clinical use. 


\section{Chapter 3 \\ Fetal ECG Extraction using Triggered Adaptive Noise Cancellation}

\subsection{Introduction}

Given the sensitivity of the in utero environment, noninvasive FECG monitoring is clearly preferable. However, as discussed in Chapter 2, an abdominal recording using surface electrodes reveals a composite signal containing an additive mixture of the FECG with unwanted interference. The dominating interference is the MECG, which is relatively stronger than the FECG.

Classic multireferenced ANC applied to FECG extraction has shown only modest results [15], but has paved the way for further research in this area. Several different approaches have since been proposed [18, 21, 23]; however, techniques have so far been restrictively applied to only the heart's electrical modality. We propose to include the heart's acoustic signal, which provides additional information regarding the fetal heartbeats.

In the classic ANC technique, a thoracic MECG is used to estimate the abdominal MECG through an adaptive filter. This is possible because these components originate from a common source, whereas the FECG component of the abdominal signal is assumed to be statistically uncorrelated since it originates from a physically independent source. However, due to the fact that the MECG and FECG overlap in the time and frequency domains, filter adaptation that is meant to attenuate the MECG component of the abdominal signal also 
partially attenuates the FECG component that occurs simultaneously. Thus, the adaptation process results in the partial cancellation of the desired signal. The proposed approach is to use the fetal phonocardiogram (FPCG) to detect the occurrence of fetal heartbeats, exploiting the intrinsic temporal correlation between the heart's mechanical and electrical activity. This modality can be acquired through established fetal heart rate monitoring techniques, such as segmentation of the FPCG acquired using an electronic stethoscope. Another more robust method of detecting fetal heartbeats is through peak detection of Doppler ultrasound data. The estimate of the temporal location of the FECG is then used to halt adaptation during a fetal heartbeat, and thus improve the estimate of the tissue filter, $h_{1}$, which is discussed in Section 2.1. The quality of the estimation of this filter, as discussed in Section 2.4 , has remained a bottleneck to improving the FECG estimate. This is the main focus of this chapter.

\subsection{Triggered Adaptive Noise Cancellation}

Triggered ANC (TANC) extends the classic ANC scheme (see Section 2.3) through an adaptation trigger input. This is useful for pulsating signals (i.e., signals that exhibit transient periods of useful information). By adapting filter taps only during periods of interference, or, conversely, preventing adaptation during periods of interest, TANC can provide a more accurate estimate of the desired signal. This concept has been shown to enhance signal estimation in [18]. Triggering is further extended in a novel technique that associates a weighting factor dependent on the period of adaptation. This technique is discussed in Section 3.2.2. 
Figure 3-1 shows the TANC scheme as applied to FECG extraction, where $w_{t}$ is the adaptation trigger, defining adaptation weighting and periods.

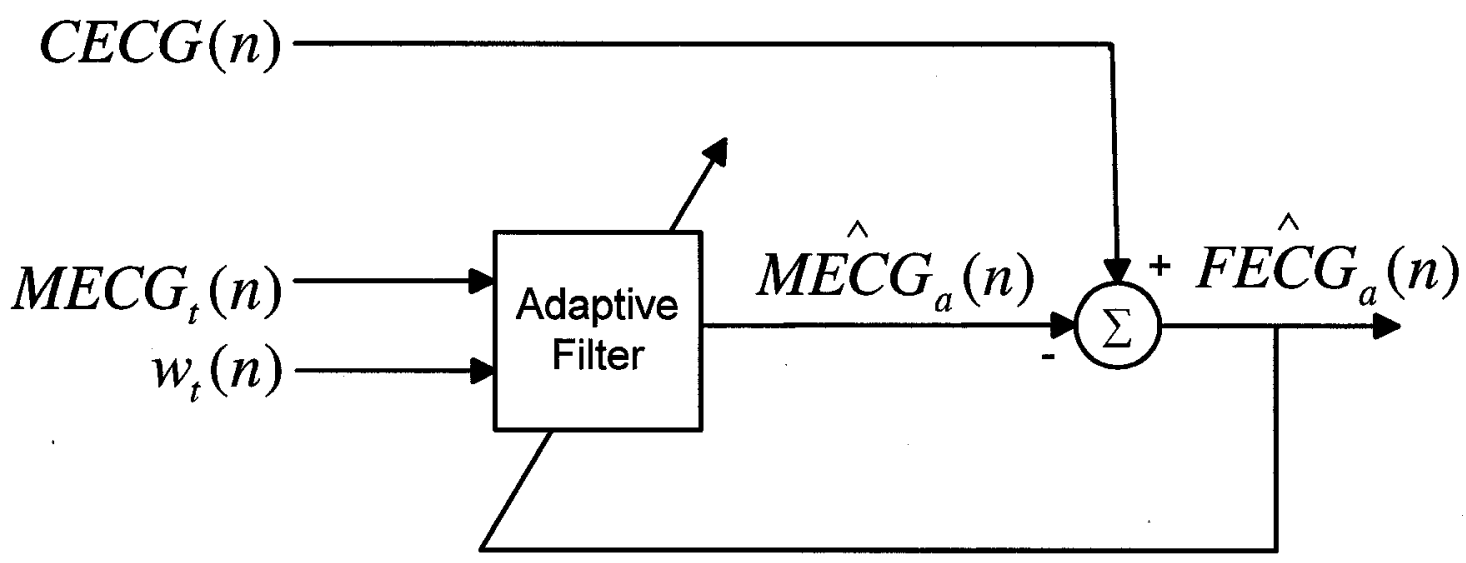

Figure 3-1 Triggered adaptive noise cancellation applied to FECG extraction

\subsubsection{Fetal Phonocardiogram Trigger}

The FPCG has been widely used for antepartum fetal heart rate monitoring $[26,27]$. Within a properly controlled setting, the FPCG can even successfully distinguish systolic and diastolic intervals of heart sounds [26]. The intrinsic temporal correlation between the FPCG and the FECG forms the basis for using the FPCG as a trigger input to the TANC scheme. This correlation in adults is well established in literature $[9,28,29]$, with a similar relationship for fetal signals. The use of the FPCG has not been incorporated into the simulation studies performed in this thesis; however, it is suggested as a possible extra modality that can be used as the trigger input to the proposed TANC scheme and its use is well justified. Other modalities, such as Doppler ultrasound may be used as well. 
By employing the use of heart sound segmentation algorithms, the first and second heart sounds of the FPCG, S1 and S2 respectively, can be localized. According to [29], S1 occurs $0.04-0.06$ s after the QRS complex, and S2 occurs towards the end of the T-wave (see Figure 3-2) in adults. The temporal relationship between the FPCG and FECG is not defined in literature, and is a topic for further research; however, it is reasonable to expect that a consistent timing relationship should exist between them since the originating processes, which are electrical and mechanical in nature, are synchronized.

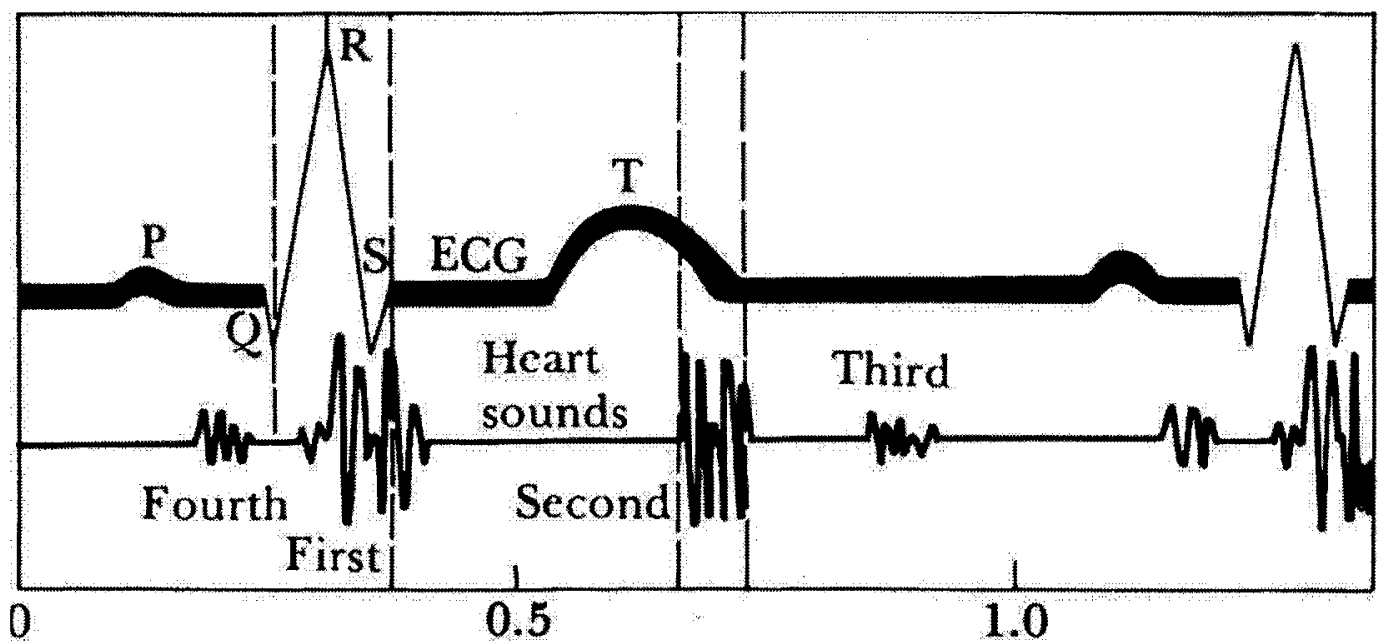

Time, s

Figure 3-2 Correlation of the ECG and the PCG, image reproduced from [9]

\subsubsection{Triggering Modes}

The TANC scheme is capable of providing a more accurate estimate of the desired signal by performing adaptation only during periods of interference and/or preventing 
adaptation during periods of interest [18]. FECG extraction performance can thus be assessed under four different triggering modes:

1. no triggering;

2. MECG adapt-enable;

3. FECG adapt-disable; and

4. dual triggering.

The MECG adapt-enable trigger is used to enable adaptation during the MECG activity since it is the MECG that is the interfering signal; adaptation is disabled between MECG pulses. Conversely, the FECG adapt-disable trigger is used to disable adaptation during FECG pulses to minimize cancellation of the FECG. Dual triggering is a combination of the two; that is, adapt only during the MECG pulse unless this coincides with an FECG pulse. The various triggering modes are illustrated in Figure 3-3.

Trigger windows are defined to be the period from the onset of the P-wave to the end of the T-wave. In this study, visual inspection of the ECGs was used to find appropriate window lengths. After automatic identification of the R-peaks using the multiplication of backward differences algorithm for QRS detection [30], trigger start and end times were chosen as $0.31 \mathrm{~s}$ before and $0.42 \mathrm{~s}$ after the R-peak for the MECG, and $0.18 \mathrm{~s}$ before and $0.25 \mathrm{~s}$ after for the FECG. These would be derived through timing relationships of ECG components [31]. 


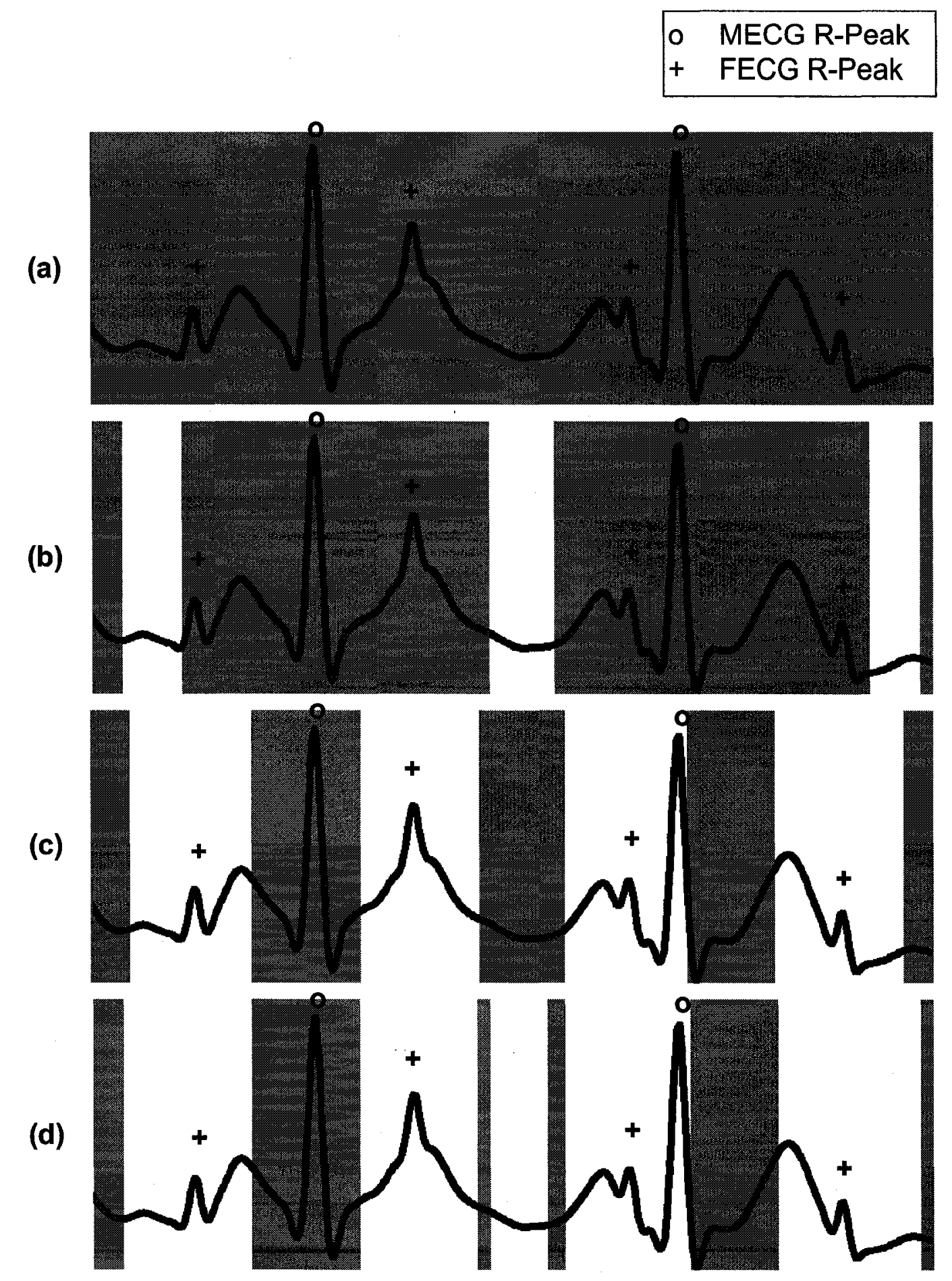

Figure 3-3 Triggering Modes (shading indicates periods of adaptation): (a) no triggering; (b) MECG adapt-enable triggering; (c) FECG adapt-disable triggering; and (d) dual triggering 


\subsubsection{Weighted Transitions}

Thus far, the concept of triggering has been referred to as an on/off switch. For example, using FECG adapt-disable triggering, the adaptive filter adapts only during periods in between FECG pulses. Adaptation is completely disabled during the FECG pulse. In this scenario, the adaptive filter may 'experience' a transient discontinuity in the adaptable data. The proposed solution is a novel form of triggering called weighted triggering. Weighted triggering smoothes the transition between adapt-enable and adapt-disable states. Various forms of smoothing can be used; for this study, we examine the performance of linear transition weighted triggering and smooth transition weighted triggering. These are illustrated in Figure 3-4.

Adapt-disable is associated with a weight of zero and adapt-enable is associated with a weight of one. The transition contains values between zero and one to control the amount of filter-tap change associated with that period. To prevent the transient effect associated with completely disabling adaptation, a small number can be used (instead of zero). Thus, adaptation will never be completely disabled; instead, the contribution of these periods to the magnitude of filter-tap changes will be small. 


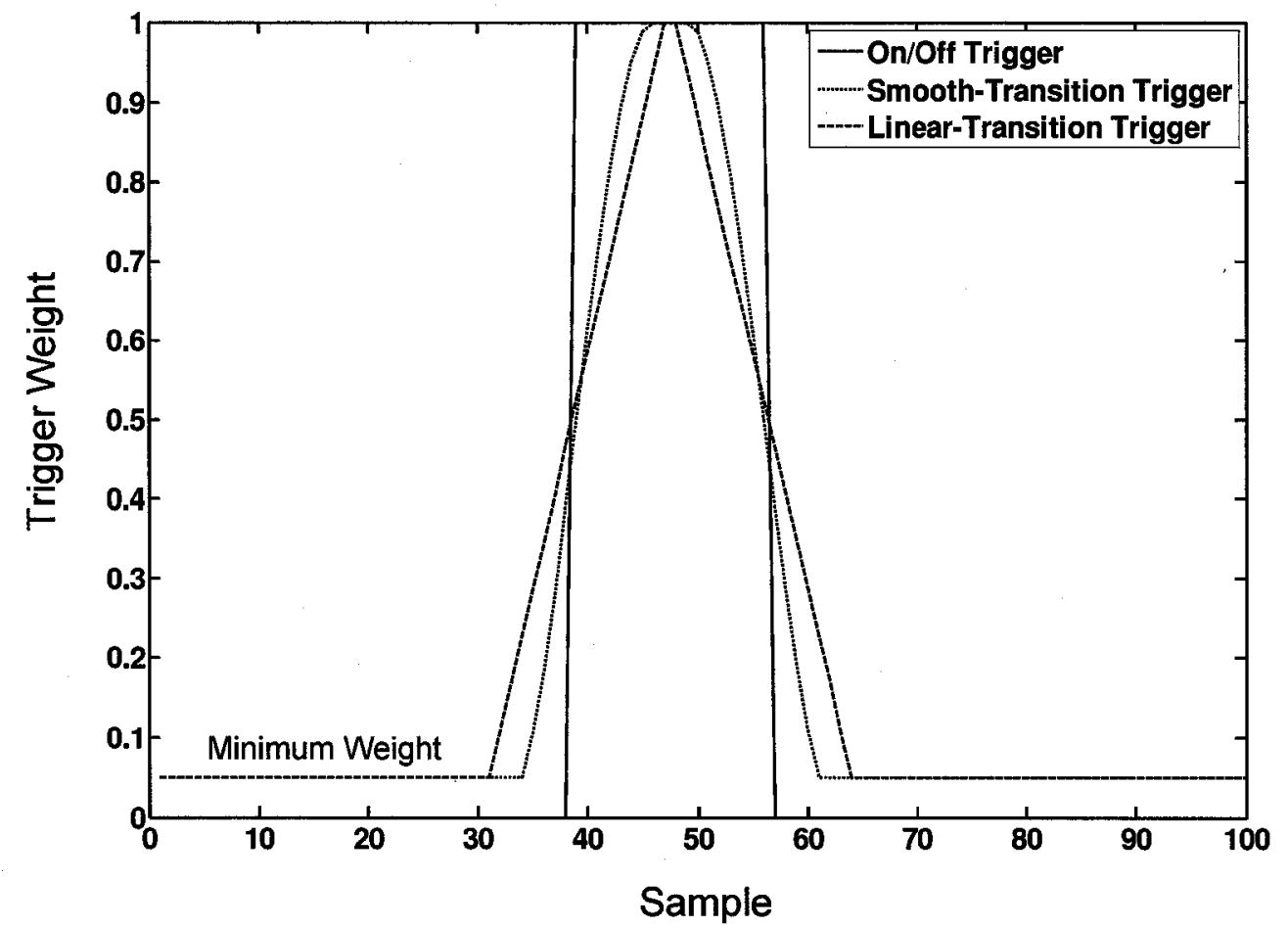

(a)

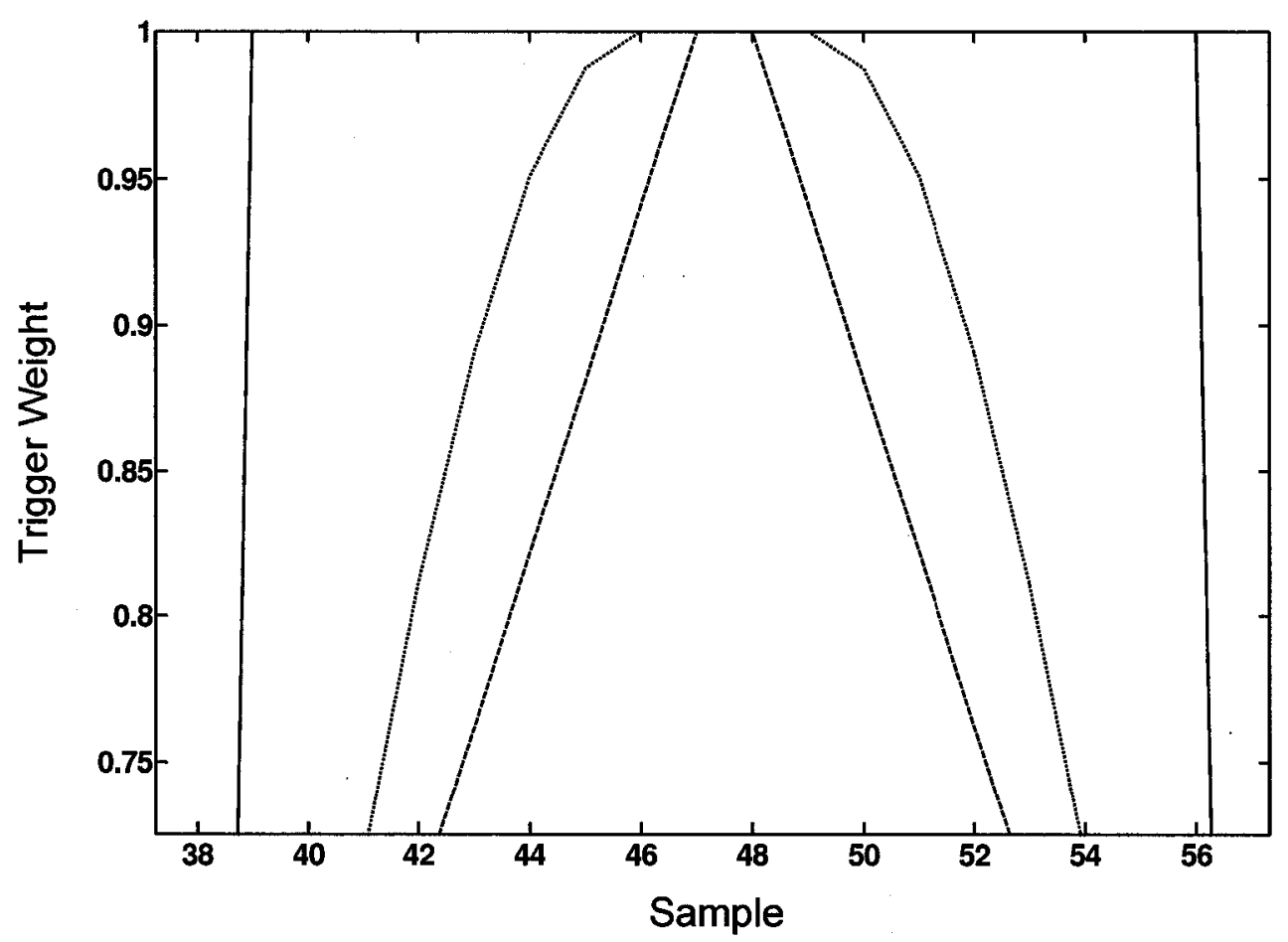

(b)

Figure 3-4 Weighted triggering in (a). Magnified view of top portion of (a) shown in (b). 35 
The use of weighted triggering in the adaptive algorithm requires the addition of an extra term to the weight-update calculation. Thus, we extend the RLS algorithm which was introduced in Section 3.2.2. The change in filter-weights is normally given by

$$
\Delta \hat{\bar{w}}(n)=\vec{k}(n) \times \xi^{*}(n),
$$

where $\Delta \hat{\bar{w}}$ represents the change in weight, $\vec{k}$ is the gain vector and $\xi^{*}$ is the complex conjugate of the cost function. An additional term, $w_{t}(n)$, which represents the trigger weight (between 0 and 1, inclusive), is then included in this formulae, i.e.,

$$
\Delta \hat{\bar{w}}(n)=\vec{k}(n) \times \xi^{*}(n) \times w_{t}(n),
$$

so that the change in weight is proportional to the period of adaptation.

\subsubsection{Linear Transition Weighted Triggering}

Linear transition weighted triggering is implemented with a linearly spaced vector from the minimum to the maximum trigger weight. The parameter required for this is a measure of the gradient of the transition. This value, $m_{t}$, is an integer that defines the number of samples before and after the transition to weight the trigger. If the chosen $m_{t}$ is greater than half the duration of a trigger's on or off period, than its maximum value is used. This constraint ensures that a transition proceeds from a minimum trigger weight to a maximum trigger weight before beginning its descent back to the minimum.

\subsubsection{Smooth Transition Weighted Triggering}

Smooth transition weighted triggering is implemented by applying a raised cosine function to the trigger windows. This function is characterized by 


$$
H_{r c}(t)=\left\{\frac{1}{2}\left\{1+\cos \left[\frac{\pi\left(t-t_{1}\right)}{2 t_{\Delta}}\right]\right\}, \quad \begin{array}{cc}
t<t_{1} \\
\min \left(w_{t}\right), & t>B
\end{array}\right.
$$

where $B$ is the duration of the desired trigger window (i.e., duration of on/off trigger + (2xduration of $\left.m_{t}\right)$ ) and $\min \left(w_{t}\right)$ is the minimum trigger weight. The parameters $t_{\Delta}$ and $t_{1}$ given in Equations 3.4 and 3.5, respectively, are functions of $m_{t}$.

$$
\begin{aligned}
& t_{\Delta}=\frac{m_{t}}{f_{s}} \\
& t_{1}=B-2 t_{\Delta}
\end{aligned}
$$

The roll-off factor of the raised cosine function is given by

$$
r=\frac{t_{\Delta}}{B-t_{\Delta}}
$$

$B$ can be varied in order to vary $r$, and thus achieve a specific $m_{t}$.

\subsection{Experimental Methods}

Experiments to test the TANC scheme proposed in Section 3.2 are carried out using synthetic ECG data. This is done so that the MECG, FECG and additive noise are welldefined. It also allows for proper quantitative analysis of the results by providing an ideal FECG for comparison.

Synthetic ECGs were simulated using [32], which describes a dynamical model for generating synthetic ECG signals. The model takes into account a realistic PQRST morphology and heart rate variability (HRV). The equations of motion describing ECG dynamics are given by three ordinary differential equations [32], 


$$
\begin{aligned}
& \dot{x}=\alpha x-\omega y \\
& \dot{y}=\alpha y+\omega x \\
& \dot{z}=-\sum_{i \in(P, Q, R, S, T)} a_{i} \Delta \theta_{i} \exp \left(-\frac{\Delta \theta_{i}^{2}}{2 b_{i}^{2}}\right)-\left(z-z_{0}\right),
\end{aligned}
$$

where

$$
\begin{aligned}
& \alpha=1-\sqrt{\left(x^{2}+y^{2}\right)}, \\
& \Delta \theta_{i}=\left(\theta-\theta_{i}\right) \bmod 2 \pi,
\end{aligned}
$$

and

$$
\theta=\arctan (y, x)
$$

From the differential equations in Equation 3.7, the $a_{i}$ coefficients determine the magnitude of the PQRST peaks, while the $b_{i}$ coefficients determine the duration of the peaks. The positions of the PQRST peaks are controlled by the $\theta_{i}$ coefficients (where $\theta$, given in Equation 3.10, is the four quadrant arctangent of the real parts of $x$ and $y$ ).

To account for HRV, the angular velocity, $\omega$, is a function of time, defined by

$$
\omega(t)=\frac{2 \pi}{T(t)},
$$

where $T(t)$ is an RR-interval time series representative of realistic HRV processes (refer to [32] for a more detailed description of a realistic HRV process). These differential equations are solved using fourth-order Runge-Kutta numerical integration, with constant time steps given by

$$
\Delta t=\frac{1}{f_{s}},
$$

where $f_{s}$ is the sampling frequency.

Baseline wander, such as that caused by respiratory interference, is given by $z_{0}$, where 


$$
z_{0}=A \sin \left(2 \pi f_{2} t\right)
$$

The respiratory frequency is given by $f_{2}$ in Equation 3.13.

Equation 3.7 generates a trajectory that models the ECG in three-dimensions, $(x, y$, $z)$, as illustrated in Figure 3-5.

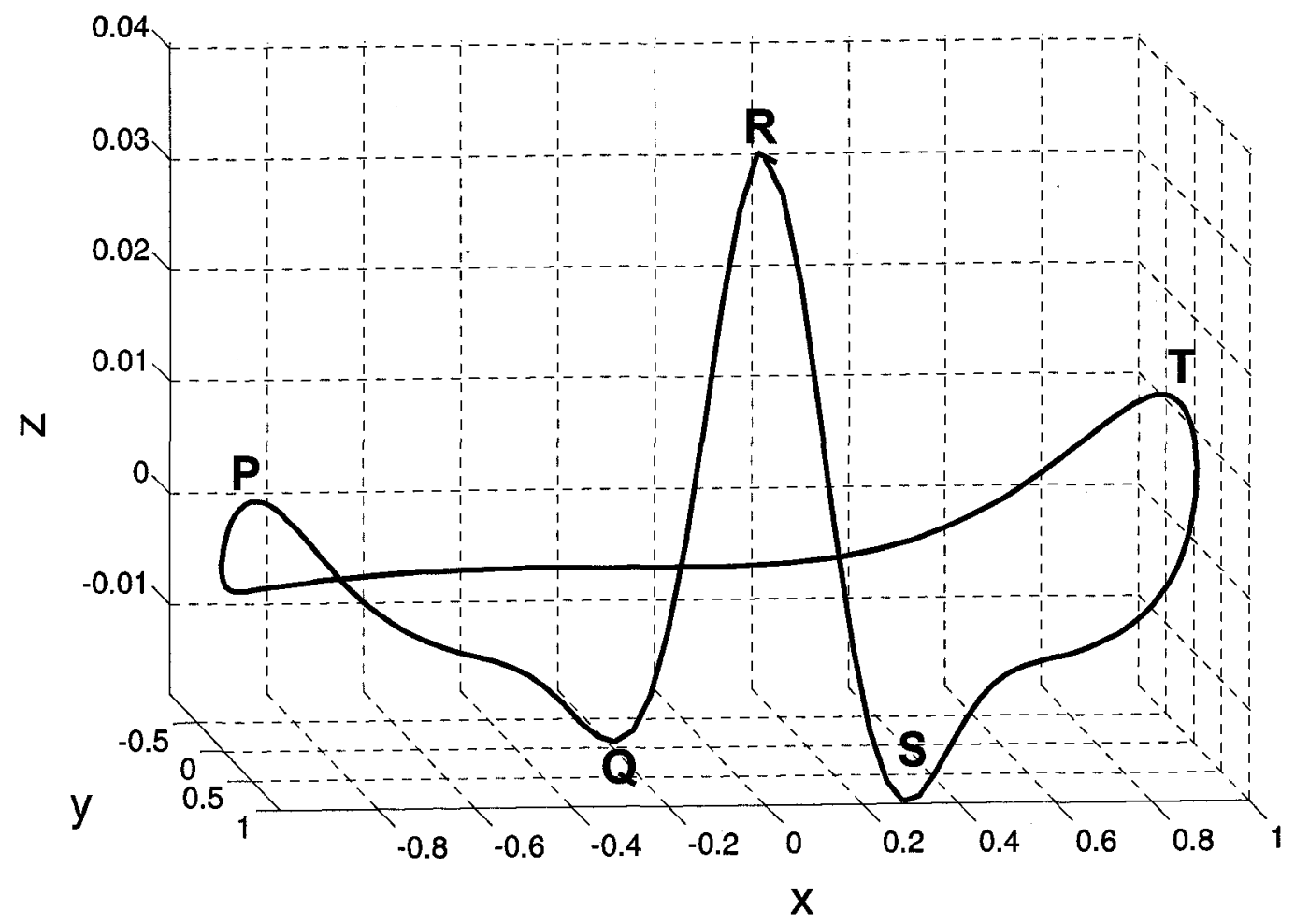

Figure 3-5 Three-dimensional state-space synthetic ECG

Synthetic ECGs were generated to represent the $M E C G_{t}$ and $F E C G_{a}$ waveforms. The parameters used to define the PQRST waveform shapes are given in Table 3-1. Figure 36 shows a single synthetic two-dimensional temporal ECG using these parameters. Other settings used for generating a train of ECG pulses are provided in Tables 3-2 and 3-3, for $M E C G_{t}$ and $F E C G_{a}$, respectively. 


\begin{tabular}{|l|c|c|c|c|c|}
\hline & $\mathbf{P}$ & $\mathbf{Q}$ & $\mathbf{R}$ & $\mathbf{S}$ & $\mathbf{T}$ \\
\hline Time [s] & -0.2 & -0.05 & 0 & 0.05 & 0.3 \\
\hline$\theta_{i}[\mathrm{rad}]$ & $-1 / 3 \pi$ & $-1 / 12 \pi$ & 0 & $1 / 12 \pi$ & $1 / 2 \pi$ \\
\hline$a_{i}$ & 1.2 & -5.0 & 30.0 & -7.5 & 0.75 \\
\hline$b_{i}$ & 0.25 & 0.1 & 0.1 & 0.1 & 0.4 \\
\hline
\end{tabular}

Table 3-1 Synthetic ECG waveform parameters

\begin{tabular}{|l|c|}
\hline Sampling Frequency & $200 \mathrm{~Hz}$ \\
\hline Mean Heart Rate & $70 \mathrm{bpm}$ \\
\hline Heart Rate Standard Deviation & $\pm 2 \mathrm{bpm}$ \\
\hline Respiratory Rate & 10 breaths $/ \mathrm{min}$. \\
\hline Peak-to-peak Amplitude & $5 \mathrm{mV}$ \\
\hline Additive White Gaussian Noise Variance & $10^{-5}$ \\
\hline
\end{tabular}

Table 3-2 Synthetic $M E C G_{t}$ settings 


\begin{tabular}{|l|c|}
\hline Sampling Frequency & $200 \mathrm{~Hz}$ \\
\hline Mean Heart Rate & $120 \mathrm{bpm}$ \\
\hline Heart Rate Standard Deviation & $\pm 4 \mathrm{bpm}$ \\
\hline Respiratory Rate & 0 breaths/min. \\
\hline Peak-to-peak Amplitude & $*$ \\
\hline Additive White Gaussian Noise Variance & $10^{-5}$ \\
\hline
\end{tabular}

* Peak-to-peak amplitude of $F E C G_{a}$ set to be 6 times smaller than that of $M E C G_{a}$, the derivation of which will be discussed shortly.

Table 3-3 Synthetic $F E C G_{a}$ settings

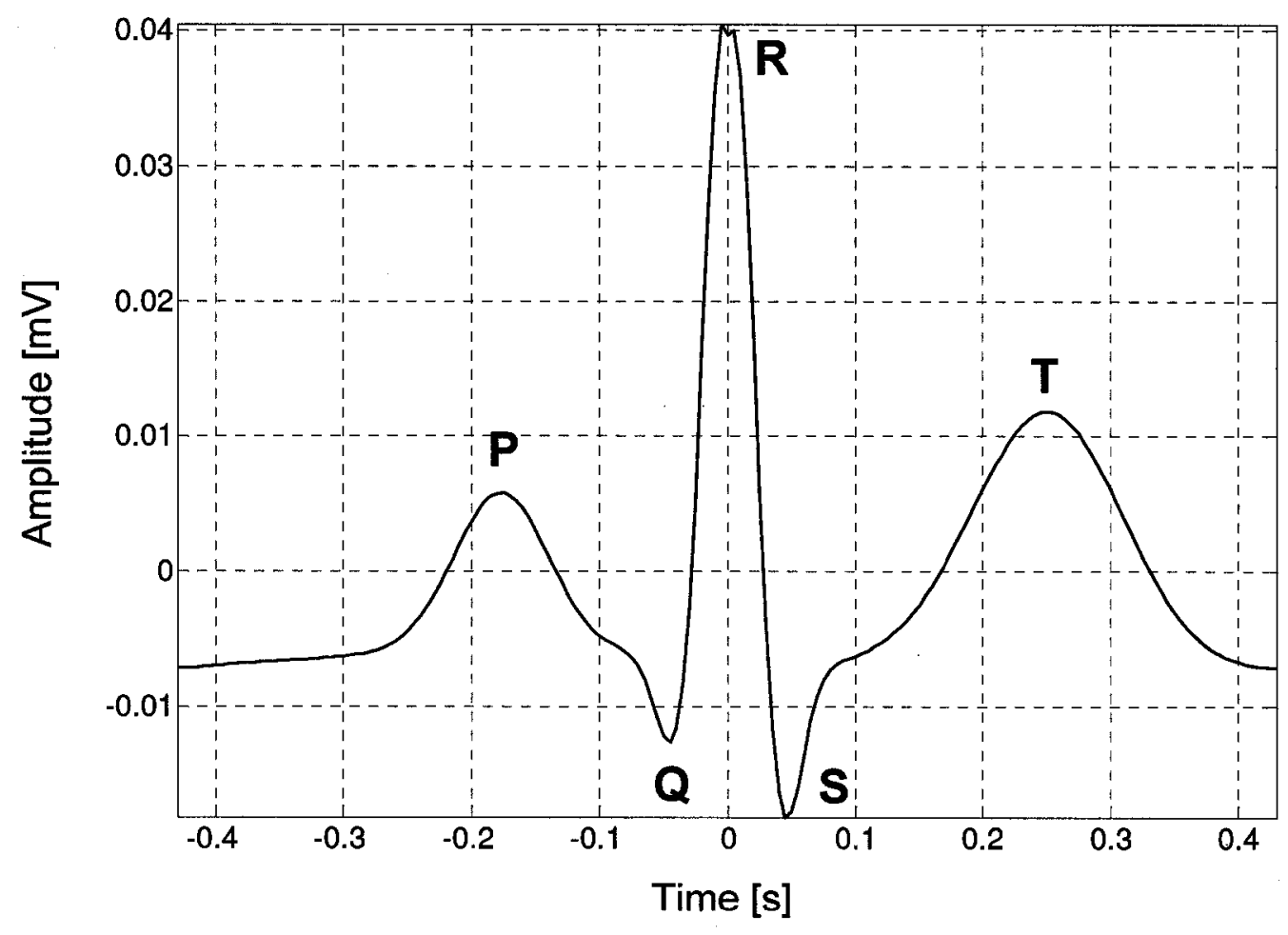

Figure 3-6 Two-dimensional synthetic temporal ECG 
To derive a relationship between $M E C G_{t}$ and the $M E C G_{a}$, the respective ECG data were collected from a subject using $\mathrm{Ag}-\mathrm{AgCl}$ surface electrodes (Red-Dot 2237, 3M Ontario, Canada) placed on the chest and abdomen, as illustrated in Figure 3-7 with channels 1 and 2, respectively. This study has been reviewed and received ethics clearance though the Carleton University Research Ethics Committee in accordance to the Tri-Council Policy Statement for Ethical Conduct for Research Involving Humans (refer to Appendix A for consent form). The ECG signal was differentially amplified using a high gain AC amplifier (Model 15A54, Grass Telefactor - Rhode Island, U.S.A.), with a gain of 1000 and bandwidth 1-100 Hz. Signals were sampled at $200 \mathrm{~Hz}$ using a 12-bit analog-to-digital converter board (PCI-6071E, National Instruments - Quebec, Canada).

Power-line interference was reduced adaptively after data collection using a $60 \mathrm{~Hz}$ reference channel. The $60 \mathrm{~Hz}$ reference channel was acquired simultaneously with the thoracic and abdominal ECG data, using no input leads. Thus the data from this channel were composed only of the $60 \mathrm{~Hz}$ sinusoidal power-line signal. Then, the classic ANC scheme, shown in Figure 2-7, was used to remove the $60 \mathrm{~Hz}$ component of the acquired thoracic and abdominal ECG data. This was done using the $60 \mathrm{~Hz}$ reference channel as the input to the adaptive filter, and the ECG data as the desired signal.

Adaptation was performed using the LMS algorithm, with $\mu=0.01$, in an FIR filter of order 14. Although an order of 2 is sufficient to model a sinusoidal signal, the presence of random noise necessitates the use of a higher order filter. The LMS algorithm was found to be more suitable for this task than the RLS algorithm. The reason for this may be that the input to the adaptive filter, i.e., the $60 \mathrm{~Hz}$ reference channel, is sinusoidal with a single 
frequency, and there is no need for memory in the system to model this quasi-deterministic signal (not fully deterministic due to random noise).

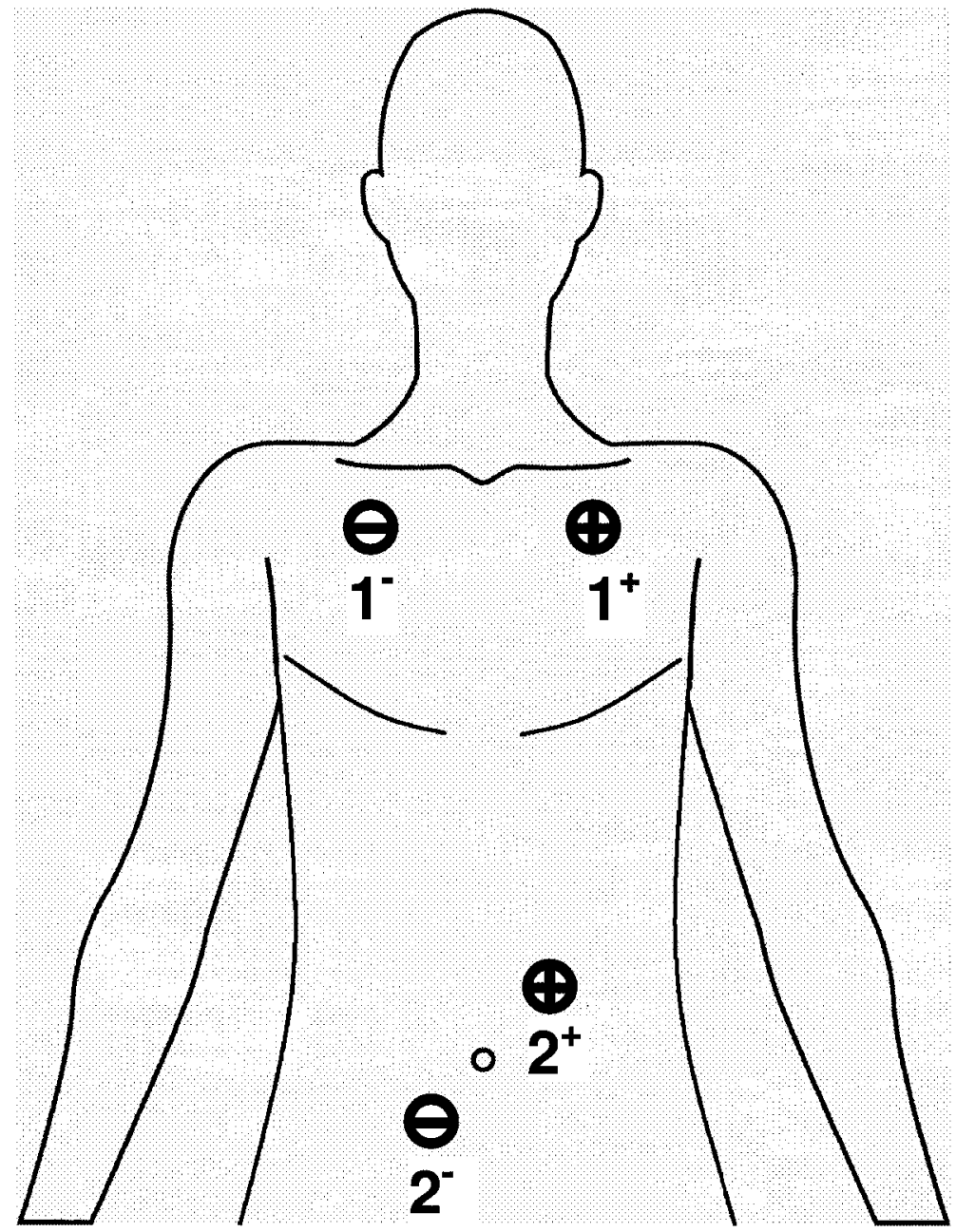

Figure 3-7 Data acquisition electrode placement 


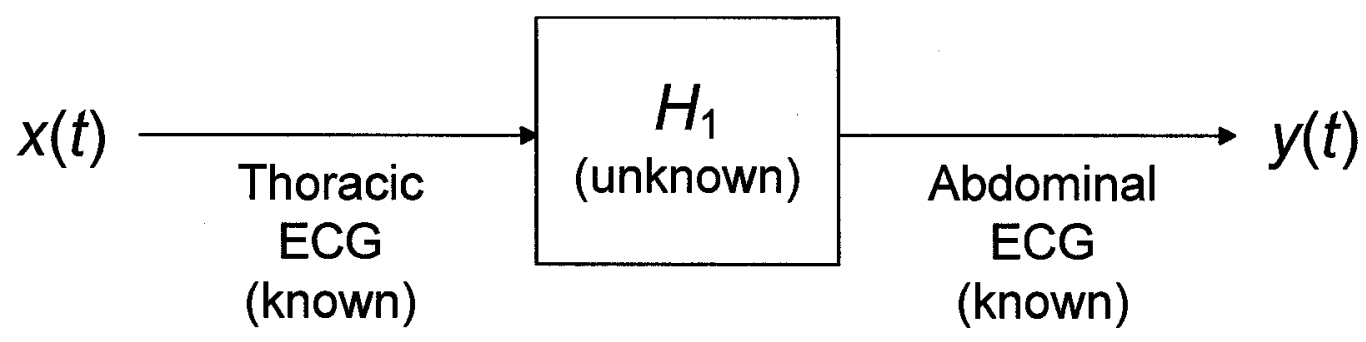

Figure 3-8 System identification problem

Defining the filter that produces the abdominal ECG output given the thoracic ECG input is a system identification problem, as shown in Figure 3-8. The magnitude of the frequency response of the unknown filter, $H_{1}$, can be found using

$$
\left|H_{1}(\omega)\right|=\sqrt{\frac{P_{y y}}{P_{x x}}},
$$

where $P_{x x}$ and $P_{y y}$ are the power spectral densities (PSD) of the input, $x(t)$, and the output, $y(t)$, respectively. These were estimated using Welch's average modified periodogram method, with a window size of 64 samples and an overlap of 63 samples (where the sampling frequency of $x(t)$ and $y(t)$ is $200 \mathrm{~Hz}$ ). Figure 3-9 shows the magnitude of the frequency response between the thoracic ECG and the abdominal ECG in the subject, i.e., | $H_{1}(\omega) \mid$. 


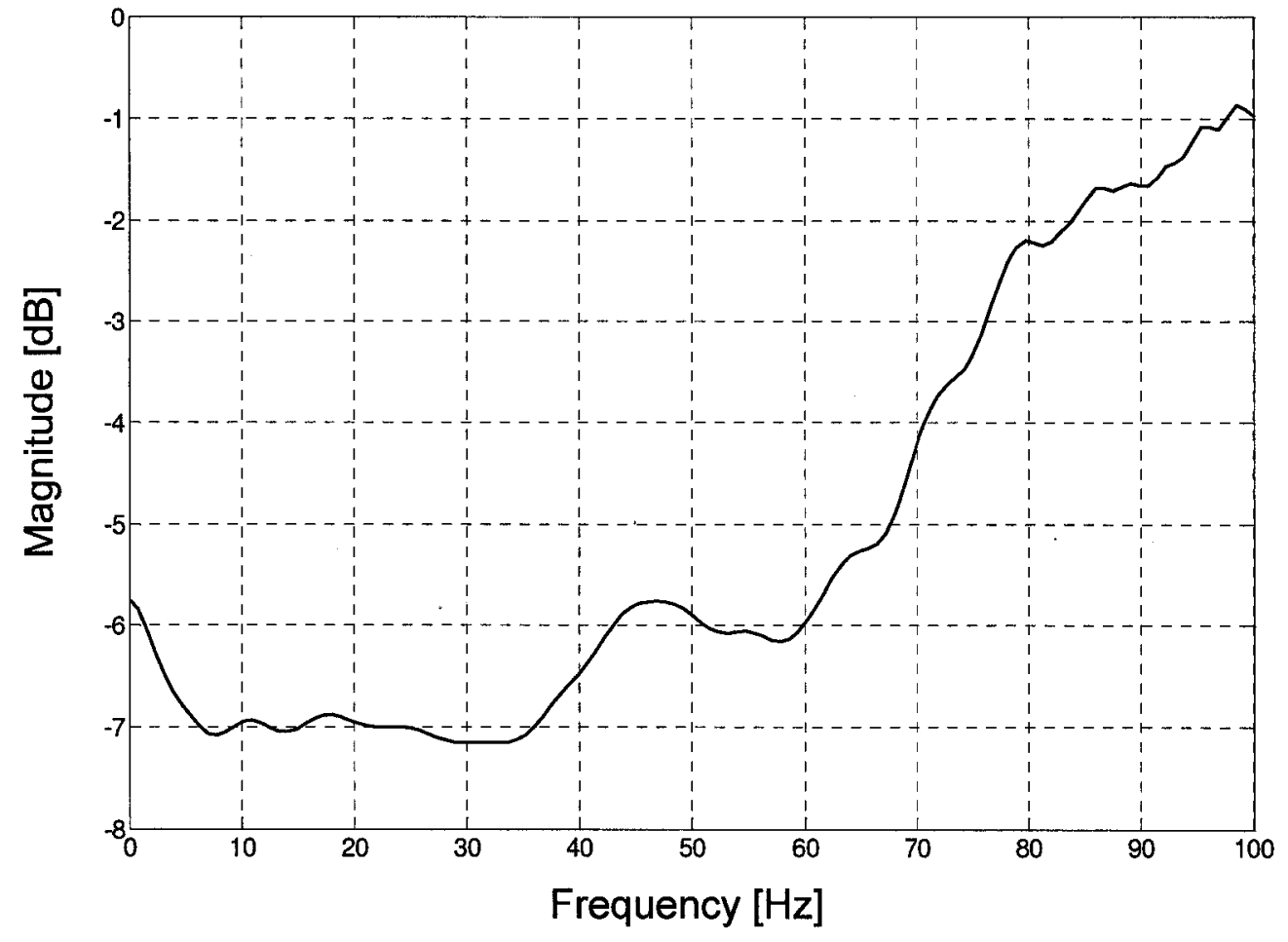

Figure 3-9 Magnitude of the frequency response between the thoracic ECG and the abdominal ECG in a subject

To simulate a synthetic $M E C G_{a}$, the noiseless synthetic $M E C G_{t}$ was passed through a linear-phase (real, symmetric coefficients) least-squares FIR approximation of $H_{1}(\omega)$, which is represented in the time-domain as $\hat{h}_{1}(t)$ in Equation 3.15. The filter output is then combined with additive white Gaussian noise (AWGN) and the noisy synthetic $F E C G_{a}$ to form $C E C G$ :

$$
C E C G(t)=\left(M E C G_{t}(t) * \hat{h}_{1}(t)+n_{g_{1}}(t)\right)+\left(F E C G_{a}(t)+n_{g^{2}}(t)\right)
$$

In Equation 3.15, $n_{g 1}$ and $n_{g 2}$ are independently generated AWGN vectors (with mean $=0$ and standard deviation $=1$ ). 
Examples of the synthetic ECG input data are shown in Figures 3-10-3-13. Note that an AWGN variance of $10^{-5}$ was initially generated and added to each signal. This value was chosen to keep the noise levels small initially, so that the performance of the system can be analyzed with high SNR inputs. Given that the power of the ideal composite signal (i.e., no AWGN) is $-16.5 \mathrm{~dB}$ and the power of the AWGN (with a variance of $10^{-5}$ ) is $-52.1 \mathrm{~dB}$, then the $\mathrm{SNR}_{\mathrm{dB}}$ of the abdominal composite data is $35.7 \mathrm{~dB}$. The effect of increasing the noise levels, and thus decreasing the SNR of the inputs will be discussed in Section 3.5.3. This noise was generated separately for each signal so that their noise components are independent.

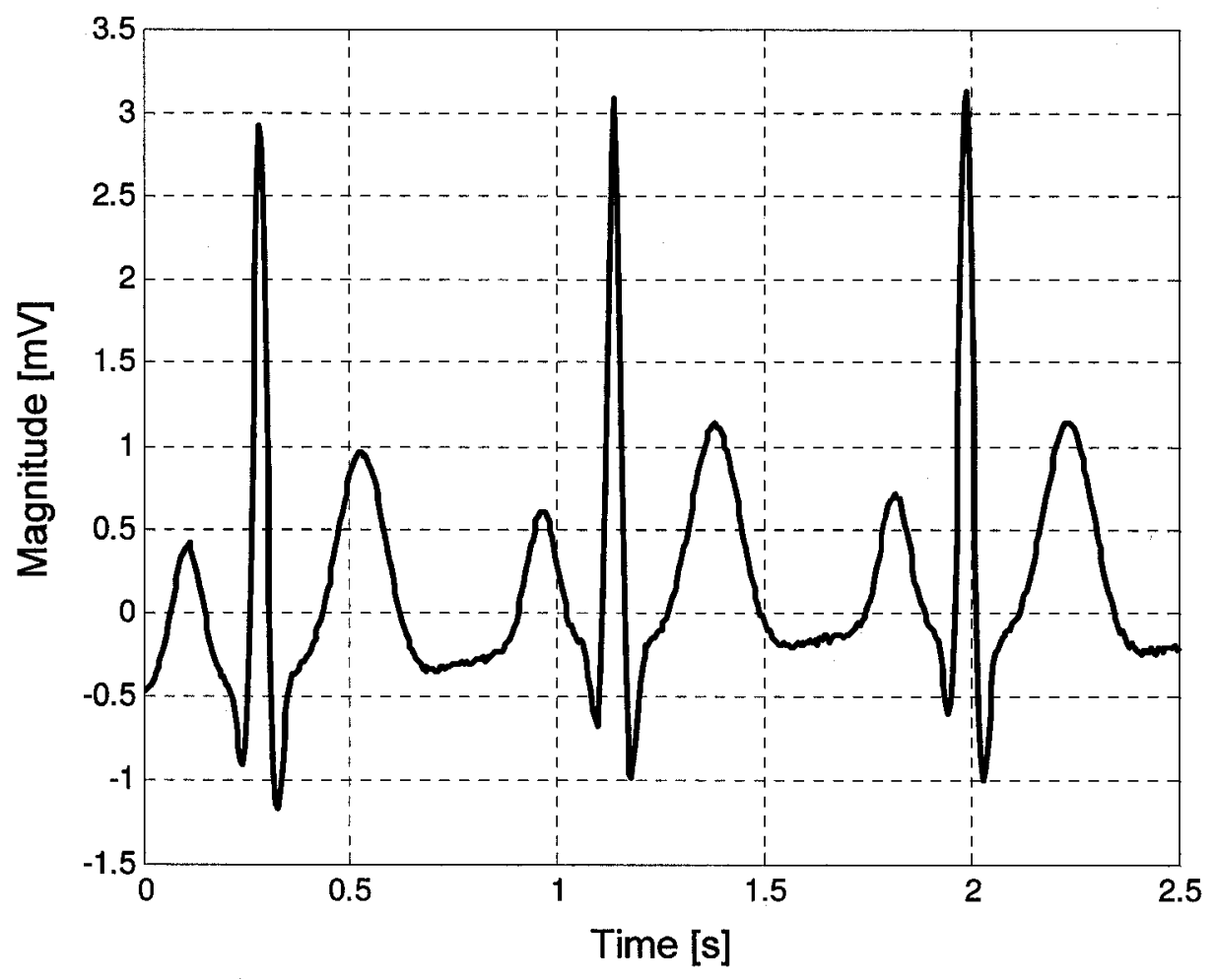

Figure 3-10 Synthetic $M E C G_{t}$ data with AWGN 


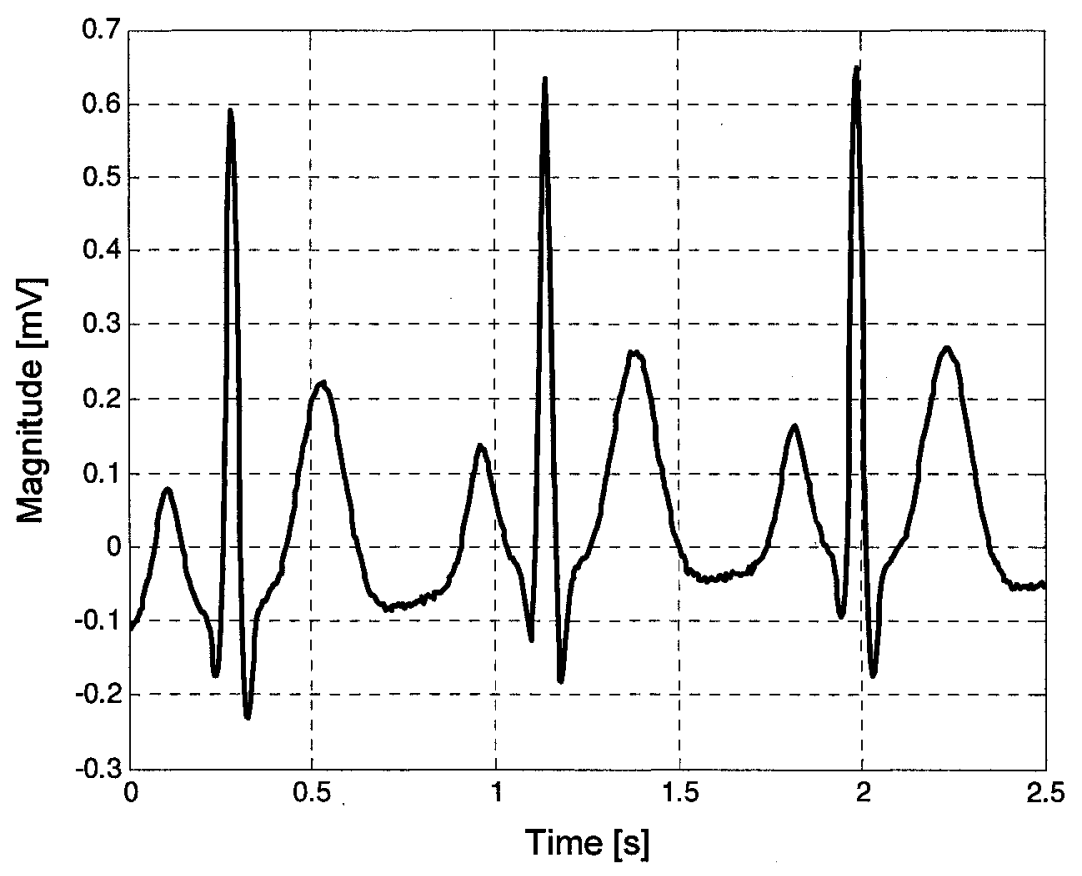

Figure 3-11 Synthetic $M E C G_{a}$ data with AWGN

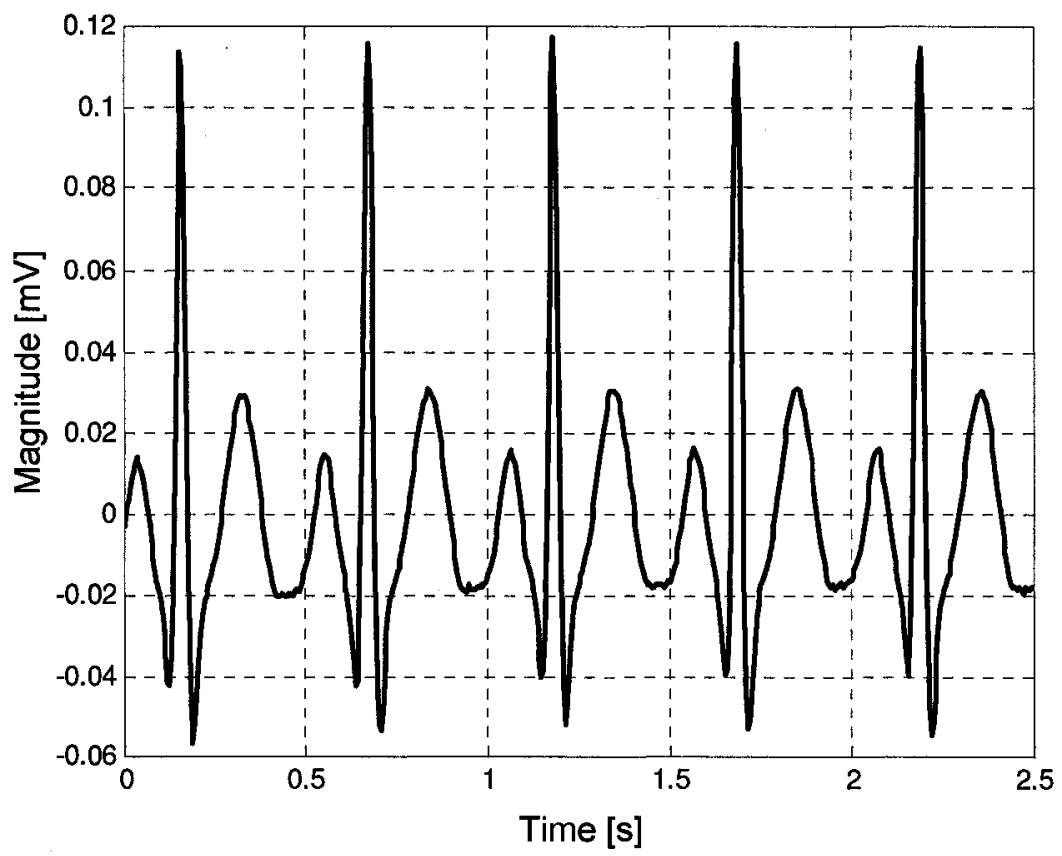

Figure 3-12 Synthetic $F E C G_{a}$ data with AWGN 47 


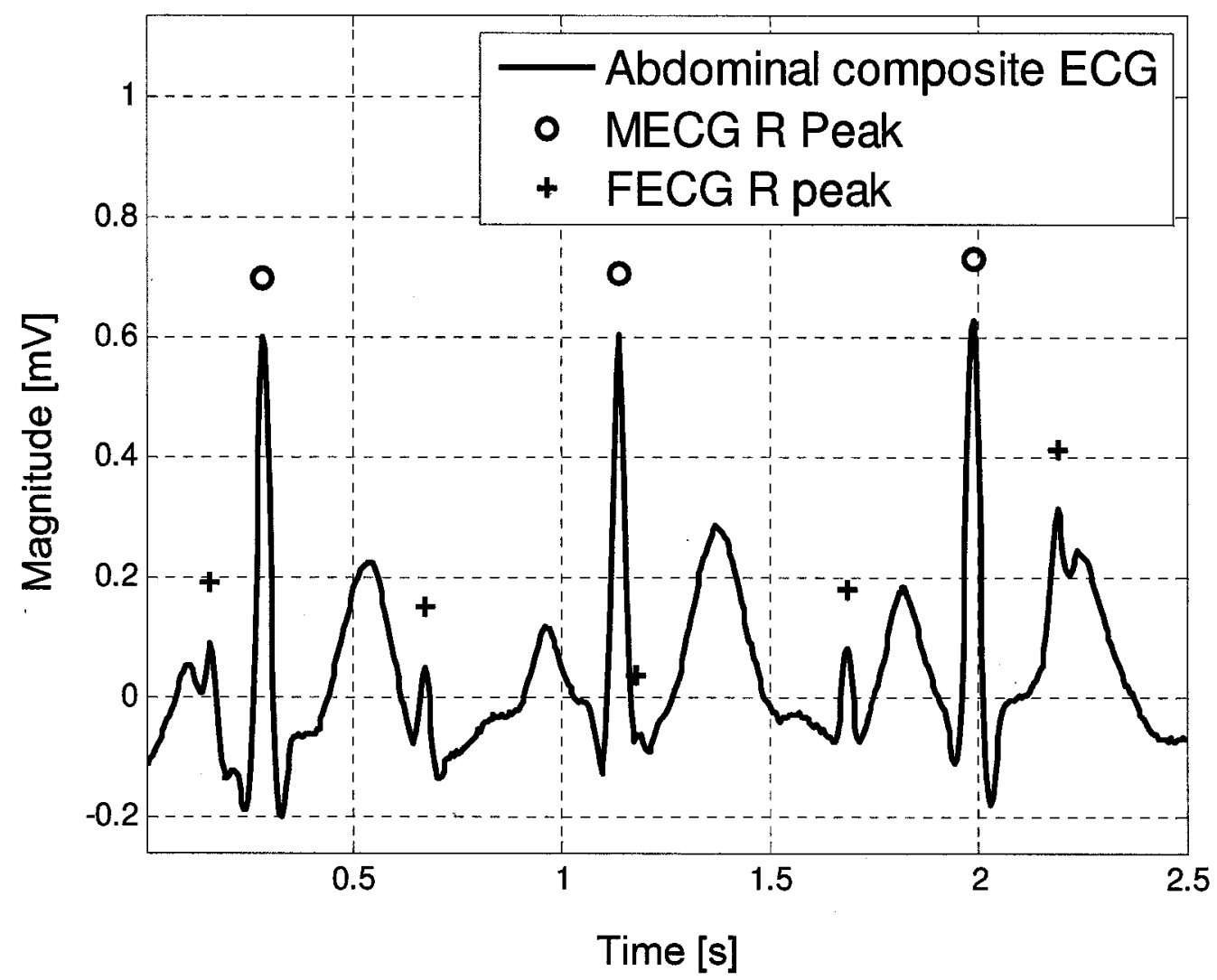

Figure 3-13 Synthetic CECG data with AWGN

\subsection{Performance Measure}

Percent residual difference (PRD) is used to quantitatively measure the performance of the system. This performance measure is commonly used to assess the quality of ECG compression techniques by comparing the decompressed ECG data to the ideal data before compression [33, 34]. The PRD quantifies the variance of the error with respect to the variance of the signal and is given by 


$$
P R D=\sqrt{\frac{\sum_{n=1}^{N}\left(x_{i}(n)-\hat{x}_{i}(n)\right)^{2}}{\sum_{n=1}^{N}\left(x_{i}(n)-\bar{x}_{i}\right)^{2}}} \times 100 \%,
$$

where $x_{i}$ is the ideal signal, $F E C G_{a}$, with mean $\bar{x}_{i}$, and $\hat{x}_{i}$ is the estimated signal, for signals of length $N$.

The PRD was calculated separately for each FECG pulse in a data set, and the results averaged. This is defined as the average pulse PRD (PPRD). The estimated signal, which consists of a train of estimated FECGs, is first divided into separate pulses. Each pulse is defined to be from the start of one P-wave to the start of the next. The pulses therefore cover the entire signal length. Each pulse is compared to the corresponding segment of the ideal FECG data using PRD. The average of all the PRDs gives the PPRD. This pulse performance measurement allows for a more in-depth analysis of the results.

\subsection{Results}

Simulations were run to compare the TANC scheme (Figure 3-1) to the classic ANC scheme (Figure 2-7). Two different adaptive algorithms were used: the LMS algorithm (see Section 2.3.1) and the RLS algorithm (see Section 2.3.2).

Initial simulations were performed to compare the results provided by the adaptive algorithms using classic ANC (i.e., no triggering). The LMS step-size, $\mu$, was set to 0.001 , with time-varying limits from $0-0.001$ and a time-varying step-size of 0.0001 . The RLS forgetting factor, $\lambda$, was set to 0.999 with time-varying limits from $0.999-0.9999$ and a time-varying step-size of 0.0001 . 
Post-lowpass filtering (LPF) was used to reduce high frequency harmonics caused by the adaptation process (i.e., gradient noise). The LPF used was designed using MATLAB's firl function, which uses windowed filter design to calculate the filter coefficients. A Hamming window was used in a digital LPF of order 32 , with a cutoff frequency, $f_{\mathrm{c}}$, of 50 Hz. This is a reasonable cutoff frequency since most of the power of the synthetic FECG lies below $50 \mathrm{~Hz}$, as seen in Figure 3-14.

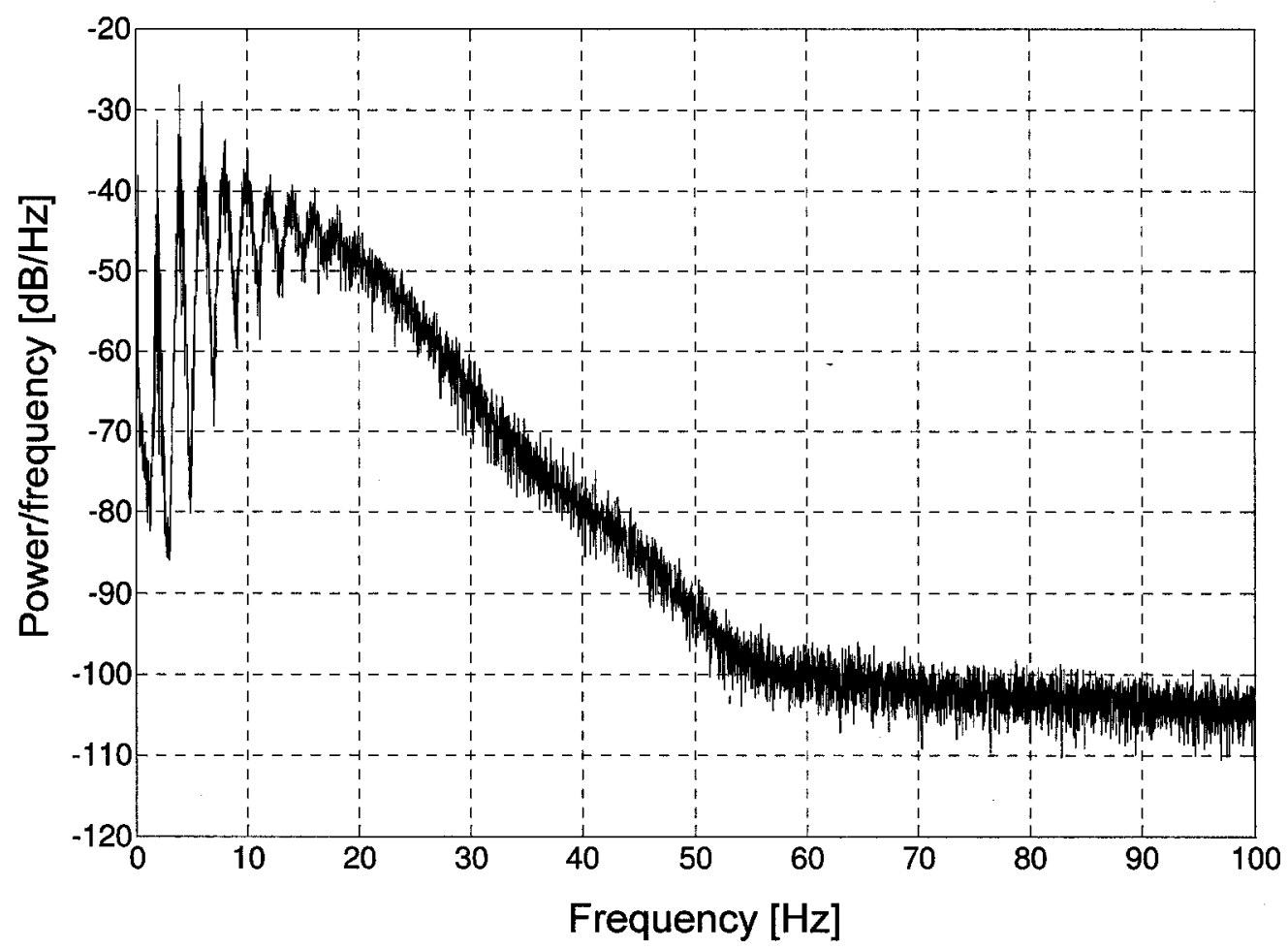

Figure 3-14 Power spectral density of the ideal synthetic FECG

The PPRD results presented in this section are based on $60 \mathrm{~s}$, or 12000 samples $\left(f_{s}=\right.$ $200 \mathrm{~Hz}$ ), of data. These data were extracted from the end portion of the estimated signal, which was a total of $300 \mathrm{~s}$, or 60000 samples, in length. Convergence of the adaptive filter 50 
was confirmed visually, and the data set used for calculating the PPRD was verified to occur after convergence.

Simulations were run for varying filter orders to identify the optimal order. Figure 315 shows the PPRD of the FECG estimate using the LMS and RLS algorithms with no triggering. The LMS algorithm gave a minimum PPRD of $24.97 \%$ while the RLS algorithm gave $13.18 \%$. The latter clearly provides a more accurate FECG estimate. This is attributed to the RLS algorithm's deterministic gradient approximation, reducing gradient noise in the output.

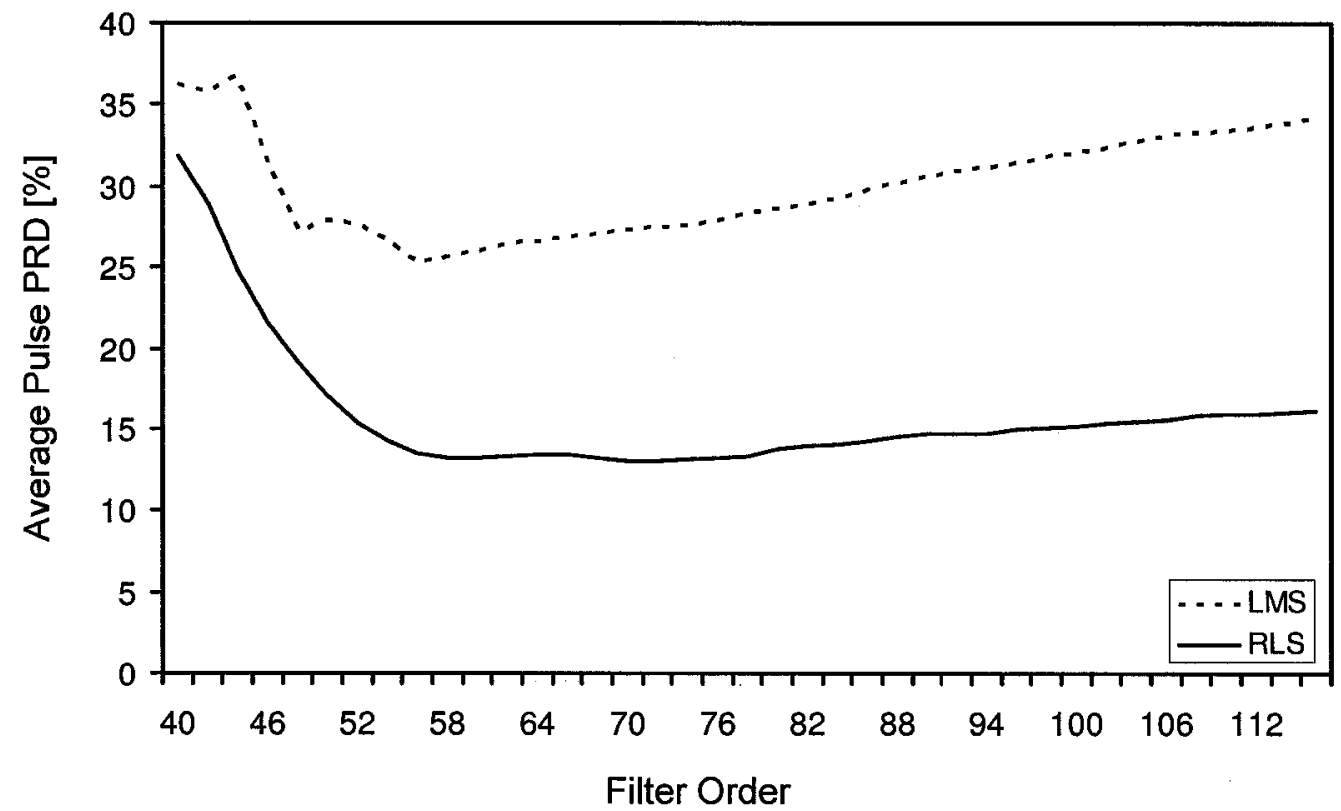

Figure 3-15 Effect of filter order on the average pulse PRD using the LMS and RLS algorithms with no triggering 


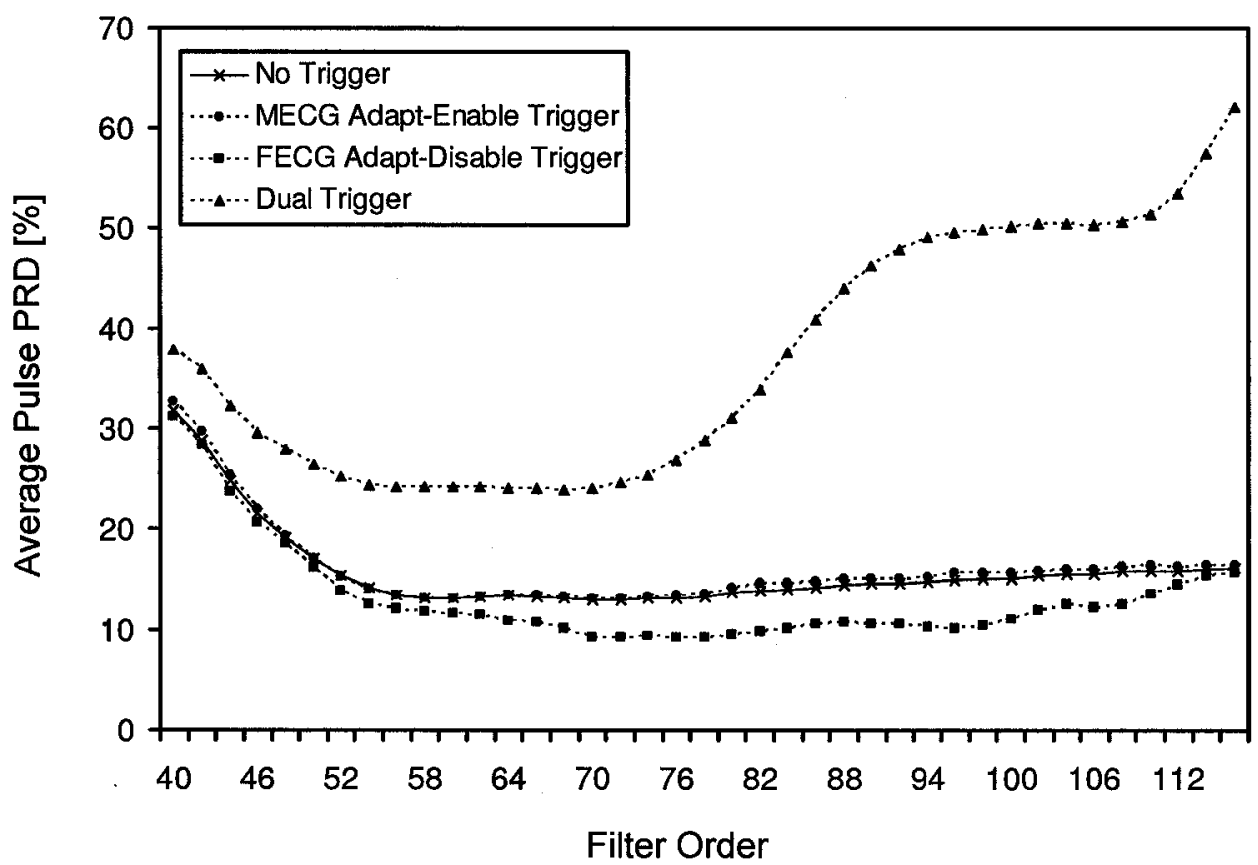

Figure 3-16 Effect of filter order on the average pulse PRD for various triggering modes using the RLS algorithm

Using the RLS algorithm, further simulations were run to compare the various triggering modes using the TANC scheme (where no triggering represents the classic ANC scheme). This was also done using various filter orders, and the results shown in Figure 3-16. A filter order of 76 using the RLS algorithm was found to be empirically optimal using classic ANC without triggering. There is a $3.8 \%$ PPRD improvement when FECG adaptdisable triggering $(\mathrm{PPRD}=9.34 \%)$ is used over the mode with no triggering $(\mathrm{PPRD}=13.18$ $\%)$.

The ideal synthetic FECG and its estimates under various triggering modes are shown in Figure 3-17 (which corresponds to the same time window as in Figure 3-13). It is not visually apparent from Figure 3-17 whether the use of triggering outperforms classic ANC. 
Given the low PPRDs (indicating high accuracy) using synthetic ECG data, any improvement in accuracy provided by triggering cannot be expected to be seen visually. However, it is an objective analysis, using PPRD, which confirms that FECG adapt-disable triggering does indeed give a better FECG estimate.

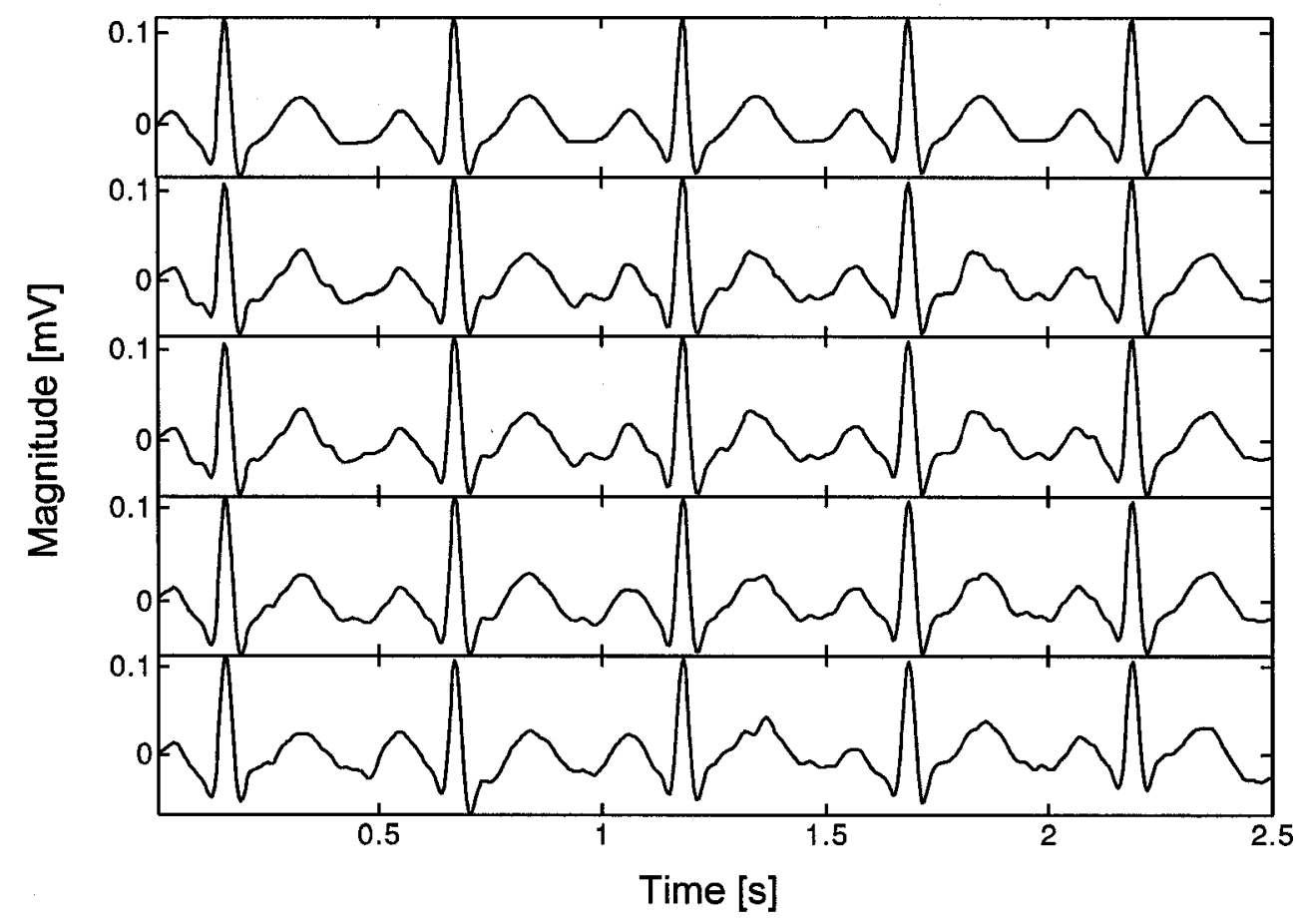

(a)

Figure 3-17 (a) Ideal FECG, and (b) FECG estimates using no trigger $(P P R D=13.18 \%)$, (c) MECG adapt-enable (PRD = 13.46 \%), (d) FECG adapt-disable $($ PPRD $=9.34 \%)$ and $(e)$ dual trigger $(P P R D=26.92 \%)$

An analysis of the individual PRDs that comprise the PPRD when using FECG adaptdisable triggering reveals that the minimal PRDs (minimum PRD $=6.08 \%$ ) occur when the FECG is less obstructed by the MECG, and the maximal PRDs (maximum PRD $=12.31 \%$ ) occur when the FECG and MECG severely overlap. It is suggested that possible future work could involve further analysis of dual triggering, since this form of triggering takes advantage 
of FECGs that do not severely overlap with the MECG, and could thus theoretically provide more accurate FECG estimates.

\subsubsection{Effect of MECG Trigger Window Size}

It is interesting to note that MECG adapt-enable triggering and, especially, dual triggering present inferior PPRDs, as shown in Figure 3-16. Given that the adaptive filter is allowed to converge before the PPRD measurements are made, the loss in performance cannot be attributed to the adaptation process. It is suspected, instead, that it is the periods of adaptation that are responsible for the poor results. In the MECG adapt-enable and dual triggering modes, the filter taps are only adapted during the MECG pulses. Since the MECG trigger window size determines these periods of adaptation, the accuracy of the FECG estimate will vary with this window size. There is even more dependency with dual triggering, since the period of adaptation is reduced further.

Figure 3-18 shows the effect of the MECG adapt-enable trigger window size on the resulting FECG estimate using the RLS algorithm with a filter order of 76. As expected, with increased window size, the dual triggering mode approaches FECG adapt-disable triggering; similarly, MECG adapt-enable triggering approaches the mode with no triggering. Thus, during the period between MECG pulses, there must be correlated information between the thoracic and the abdominal signals. This implies that the MECG pulse extends into this interpulse interval. Figures 3-10 and 3-11, which show the synthetic thoracic and abdominal MECG data, respectively, reveal that there exists very little, if any, dead space in between the pulses. This is normally not the case using real data, where the ECG pulses are clearly 
separated. This lack of separation between MECG pulses may be the cause of the poorer performance exhibited by both MECG adapt-enable triggering and dual triggering.

Figure 3-18 also shows that with a large enough window size, dual triggering does exhibit an improvement over FECG adapt-disable triggering; however, the performance gain is relatively small (0.41\% PPRD) and may not justify using MECG adapt-enable triggering, due to the added complexity. Thus, attenuation of the abdominal MECG component is sufficient with a TANC scheme using only FECG adapt-disable triggering. Further research examining the effects of MECG adapt-enable triggering and dual triggering is suggested using real data.

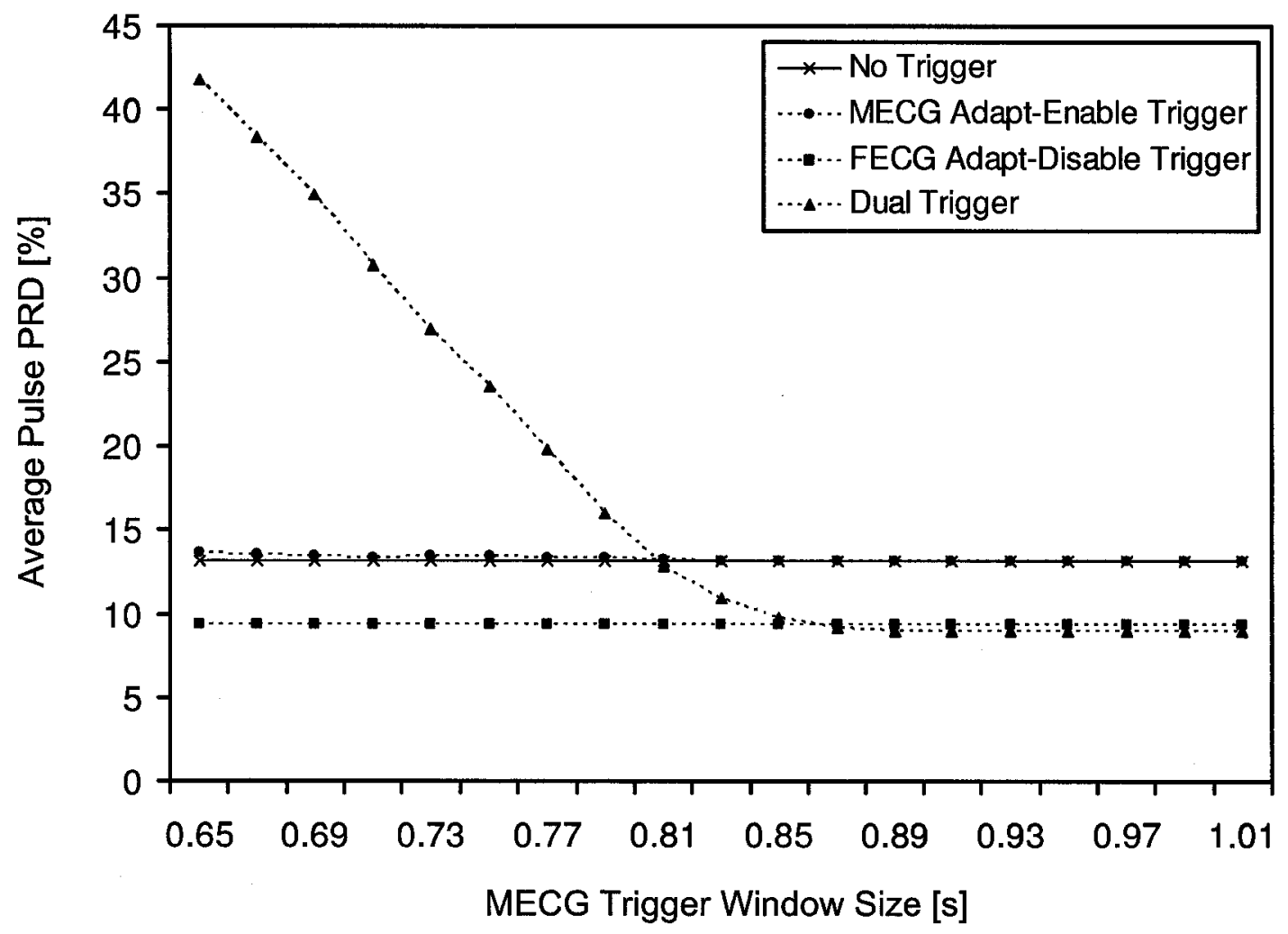

Figure 3-18 Effect of MECG trigger window size on the average pulse PRD 


\subsubsection{Effect of Weighted Triggering}

Now that we have established that the TANC scheme (using the RLS adaptive algorithm with FECG adapt-disable triggering and on/off transitions) does indeed provide a more accurate FECG estimate than the classic ANC scheme, we want to analyze the effect of using various transitions with weighted triggering (see Section 3.2.2). We examine the effect of using smooth and linear transitions by varying the $m_{t}$ parameter (i.e., the number of samples before an after an on/off transition to weight the transition).

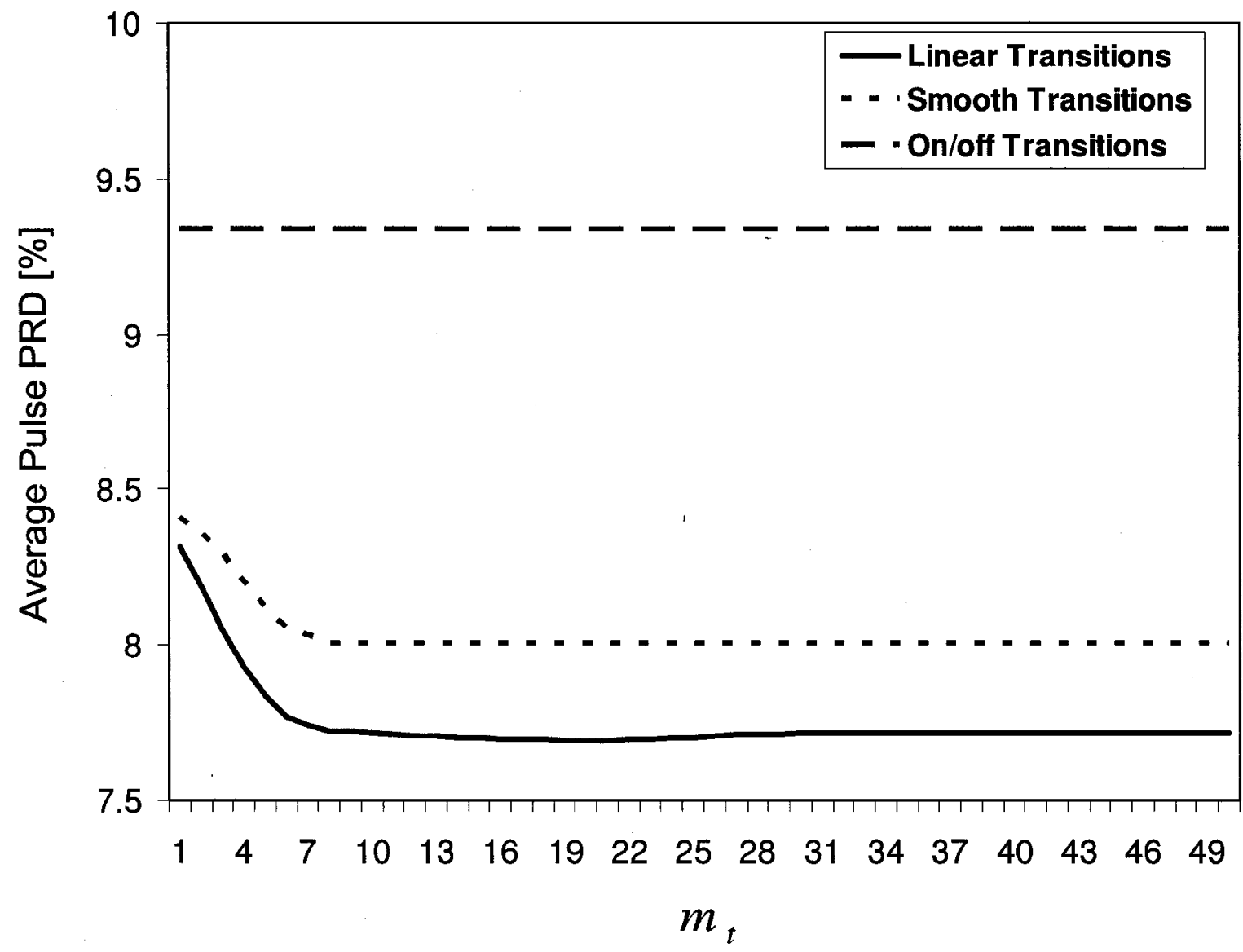

Figure 3-19 Effect of $m_{t}$ on the FECG estimate 
Figure 3-19 shows the PPRD on the resulting FECG estimate using the RLS algorithm with FECG adapt-disable triggering with smooth and linear transitions. The previous result using on/off transitions is also plotted in this figure for comparison. A minimum trigger weight of 0.05 , which was initially found to be empirically optimal, was used. The use of a minimum trigger weight ensures that adaptation is never completely disabled, only its effects are reduced during the FECG.

Both smooth transitions and linear transitions give better PPRD results compared to on/off transitions. As shown in Figure 3-19, linear transitions outperformed smooth transitions. Compared to the previously shown PPRD result of $9.34 \%$ with on/off transitions (see Figure 3-16), linear transitions improved the PPRD by $1.65 \%$, giving a minimum PPRD of $7.69 \%$ with $m_{t}=20$. The effect of increasing $m_{t}$ further does not increase the PPRD significantly, stabilizing at $7.71 \%$. Given that the average adapt-disable window length in the ECG data is 87 samples, and the average adapt-enable window length is 13 samples, the maximum $m_{t}$ would be about 6 (i.e., 6 samples before the transition, and 6 afterwards). It can be seen from Figure 3-19 that the improvement of the PPRD after $m_{t}=6$ is quite small. This is due to the constraint that limits the value used as $m_{t}$ to a maximum based on the window length; which is illustrated in Figure 3-20. In this figure, $m_{t}=20$ is used, but it can be seen that the slope of the transition is constrained by the period of the adapt-enable window, and adjusts to the various window lengths. 


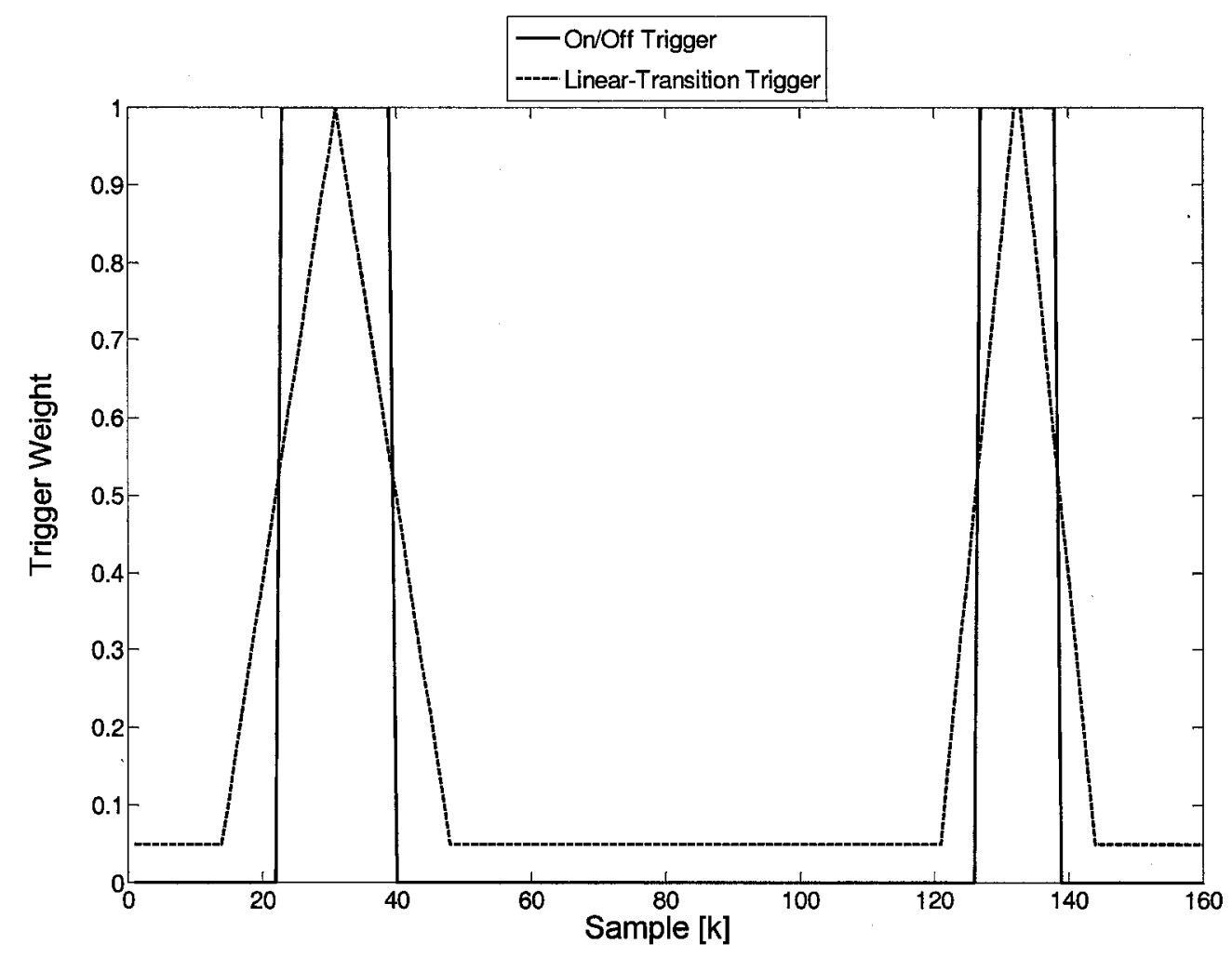

Figure 3-20 Linear transition triggering with maximum $m_{t}$ constraint

The performance provided by FECG adapt-disable linear transition weighted triggering is a $5.49 \%$ PPRD improvement over classic ANC with no triggering. Figure 3-21 shows the ideal FECG and its estimates using classic ANC with no triggering and TANC using FECG adapt-disable weighted triggering with on/off and linear transitions. 


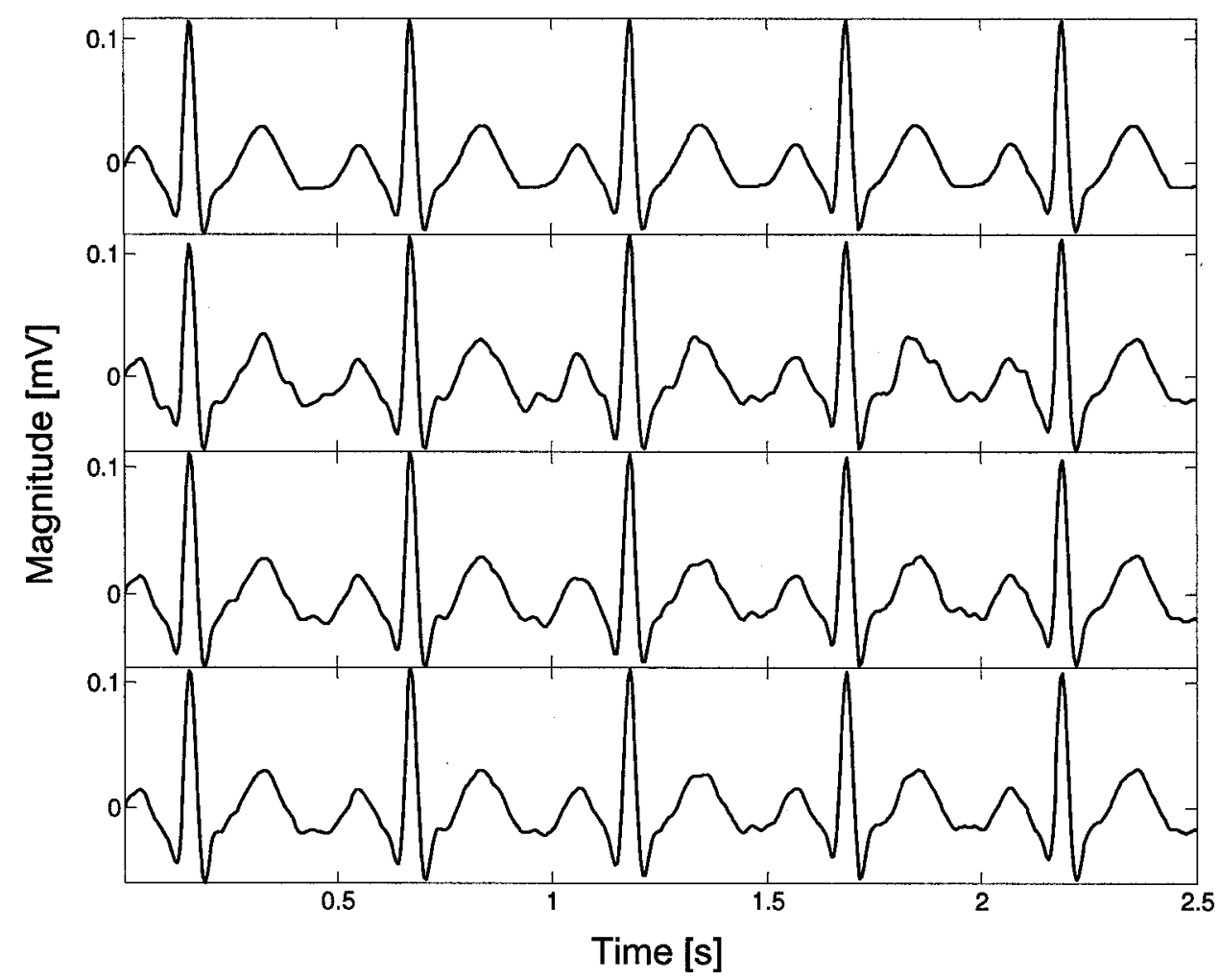

(a)

(b)

(c)

(d)

Figure 3-21 (a) Ideal FECG, and (b) FECG estimates using no trigger (PPRD =13.18 \%), (c) FECG adapt-disable with on/off transitions (PPRD $=9.34 \%)$ and (d) FECG adaptdisable with linear transitions (PPRD $=7.69 \%$ )

\subsubsection{Effect of Lowering the SNR of the Synthetic Data}

Further simulations were run with increased noise levels to analyze the system's robustness. It is assumed that the thoracic MECG reference signal can be acquired with a higher SNR. This is a reasonable assumption since the thoracic signal contains little MES interference from intra-abdominal vibrations and fetal movements. The AWGN variance in the thoracic MECG is kept constant at $10^{-5}\left(\mathrm{SNR}_{\mathrm{dB}}=35.7 \mathrm{~dB}\right.$, as shown in Section 3.3). In contrast, the abdominal composite signal may suffer from the above mentioned interferences. 
The AWGN variance in this signal is varied from $10^{-5}$ to $10^{-4}\left(\mathrm{SNR}_{\mathrm{dB}}\right.$ varies from $35.7 \mathrm{~dB}$ to 25.7 dB) and the effect on the FECG estimate is shown in Figure 3-22.

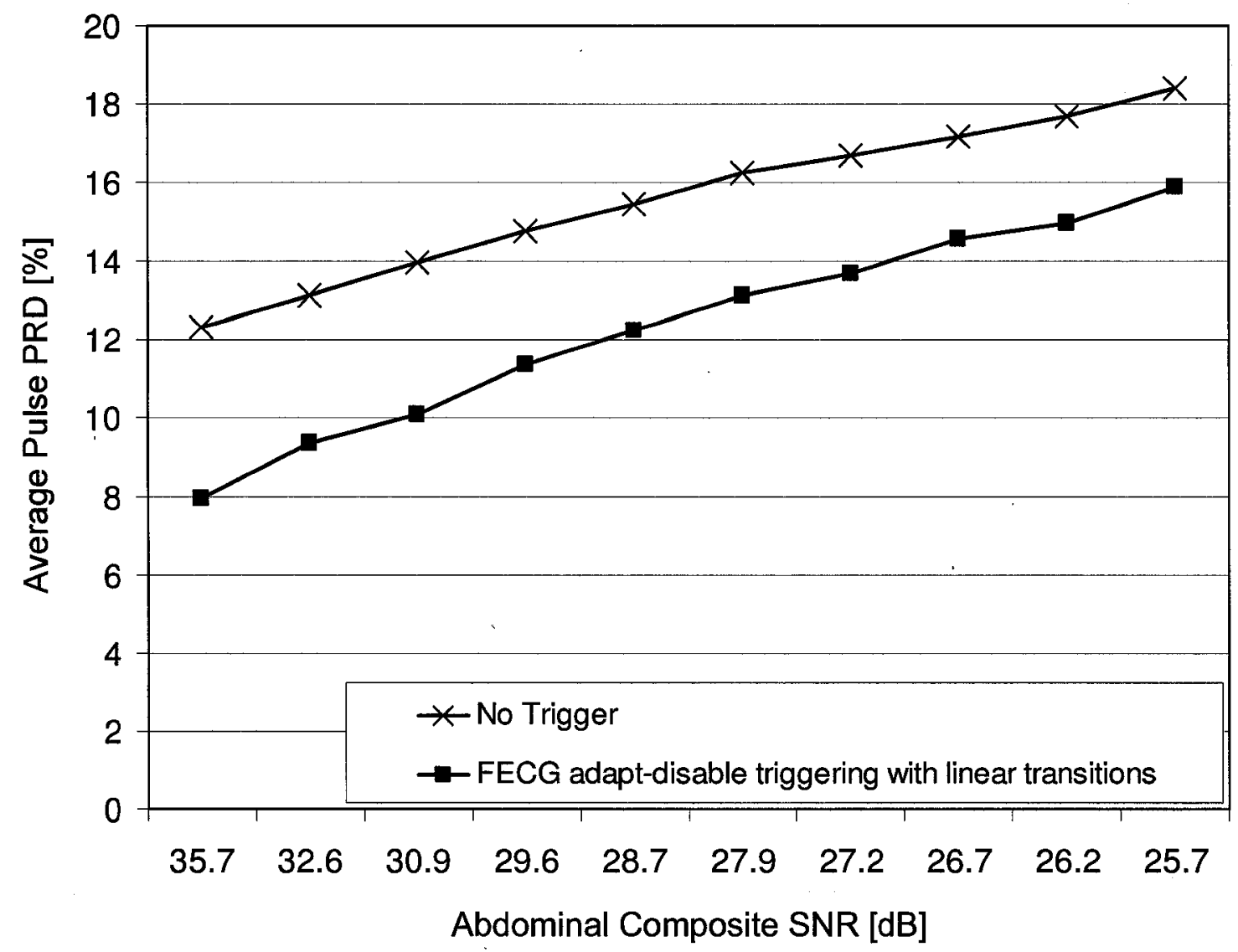

Figure 3-22 Effect of the abdominal composite SNR on the FECG estimate

As expected, the effect of added noise deteriorates the accuracy of the estimated FECG. In the presence of low SNRs, the diagnostic usefulness of FECG extraction using single-primary channel ANC is diminished. However, note that the use of FECG adaptdisable triggering (with linear transitions) consistently improves the FECG estimate. Analysis of the PSD of the error between the ideal FECG and its estimate reveals that the 
error is white noise. Thus, the SNR of the abdominal signal can be increased by recording multiple abdominal channels, assuming uncorrelated noise from the various measurement sites. This is the topic of Chapter 4.

\subsection{Conclusions}

In this chapter, we have proposed to use a TANC system for the extraction of the FECG from a noninvasively acquired abdominal composite signal. This can be realizable through the use of an extra modality, such as the FPCG, that can be used as an adapt-disable trigger. Due to the intrinsic temporal correlation between the heart's mechanical and electrical activity, the FPCG can be used to derive the temporal occurrence of FECG pulses. By preventing adaptation during such periods within the primary signal, we have shown that the adaptation process arrives at a more accurate result than with classic ANC. Indeed, a PPRD improvement of $3.80 \%$ is attained. This result is based on data obtained from a single subject for derivation of the thoracic-abdominal transfer function. To generalize the results obtained to a larger population is suggested as future research. This is critical since the thoracic-abdominal transfer function will differ between subjects, thus affecting the system performance. The results obtained in this study still conform to the objective of this thesis by demonstrating the advantage of using a TANC scheme. Performing adaptation only during periods of interference, i.e., MECG pulses, was also investigated and the slight improvement gained by using MECG adapt-enable triggering does not justify its use.

Furthermore, by using linear transition weighted triggering, instead of on/off transitions, the PPRD of the FECG estimate was found to be $5.49 \%$ better than using classic 
ANC. This technique reduces the transient discontinuity effect that is associated with the immediate and complete disabling of adaptation.

Although the use of weighted triggering has improved the estimate of the tissue-effect filter resulting in a better estimate of the FECG, the technique still requires an input with a reasonable SNR. A technique to improve the SNR of the abdominal composite signal is needed to provide a better FECG estimate in the presence of noise. Next, we investigate the use of multiple abdominal channels coupled with a second enhancement stage (following TANC) to this end. This will be discussed in Chapter 4. 


\section{Chapter 4 \\ Multichannel Fetal ECG Extraction and Enhancement}

\subsection{Introduction}

In Chapter 3, we examined the use of TANC with weighted transitions and showed, using synthetic ECGs, that it provides a better estimate of the FECG compared to classic ANC. While the MECG is the major source of interference in the abdominal composite signal, other sources of noise exist that are problematic as well. These additional additive noise sources include muscular and respiratory interference, thermal noise (from electronic equipment), and the electrode-skin interface. Thus, while removing the MECG is important, there is still the need to further improve the SNR from other noise sources. In this regard, we now extend the system to include the acquisition of multiple abdominal channels, where it is assumed that the noise between the channels is uncorrelated. Multichannel FECG extraction is not a new concept. Widrow's early work used a multireferenced ANC scheme, where multiple thoracic channels were acquired [15]. Following this, the use of a time-sequenced adaptive filter (see Section 2.3) with two abdominal channel inputs was used for FECG enhancement [18]. More recently, BSS techniques (see Section 2.4) also used multiple abdominal channels $[21,22]$.

In this chapter, we exploit data from multiple abdominal channels to perform TANC with weighted transitions for FECG extraction, followed by adaptive system identification 
(ASI) for FECG enhancement. To test this two-stage process, we analyze a case study where an external stimulus pulse (SP) is applied to a subject's back and acquired at multiple recording sites on the abdomen; this SP is used to simulate the FECG. Thus, extraction is performed on real signals, albeit using SPs as the signal we wish to extract. This allows testing of adaptive filtering on a time-varying thoracic-abdominal transfer function, as opposed to the static one used in chapter 3.

\subsection{Two-Stage Multichannel System Design}

The TANC scheme described in Section 3.2 will be used for each abdominal channel as the first stage in this multichannel system for FECG extraction. Using the best estimate of the SP provided by the first stage, which is chosen using the performance measure criteria described in Section 4.4, the data are run through a second stage of triggered ASI (TASI) to exploit independent noise within the multiple channels and arrive at a cleaner estimate (i.e., FECG enhancement). The final FECG estimate, and output of the system, is calculated as the ensemble average of the outputs of stage 2 .

\subsubsection{Stage 1: Fetal ECG Extraction using Triggered Adaptive Noise Cancellation}

The TANC scheme, shown in Figure 3-1, with linearly weighted transitions was used in this study to attenuate the MECG interference; thus extracting the desired pulse. Figure 3-1 represents the TANC scheme for single channel inputs (i.e., single thoracic and single abdominal channels). Stage 1, as shown in Figure 4-1, uses multiple independent TANC 
blocks. These blocks (each of which contains Figure 3-1) receive a common $M E C G_{t}$ input; however, each block attempts to attenuate a different $M E C G_{a}$ waveform within each of the acquired abdominal composite signals. The abdominal signal vectors are illustrated by inputs $C E C G_{1}-C E C G_{N}$ and the trigger by $w_{t}^{1}$. The outputs of this stage are $\mathrm{N}$ estimates of the desired pulse, represented by $\hat{F E C G_{a 1}}-\overrightarrow{F E C G_{a N}}$, where $\mathrm{N}$ is the number of abdominal channels acquired. These $\mathrm{N}$ outputs of stage 1 form the inputs for stage 2. Note that superscripts are used to denote the stage.

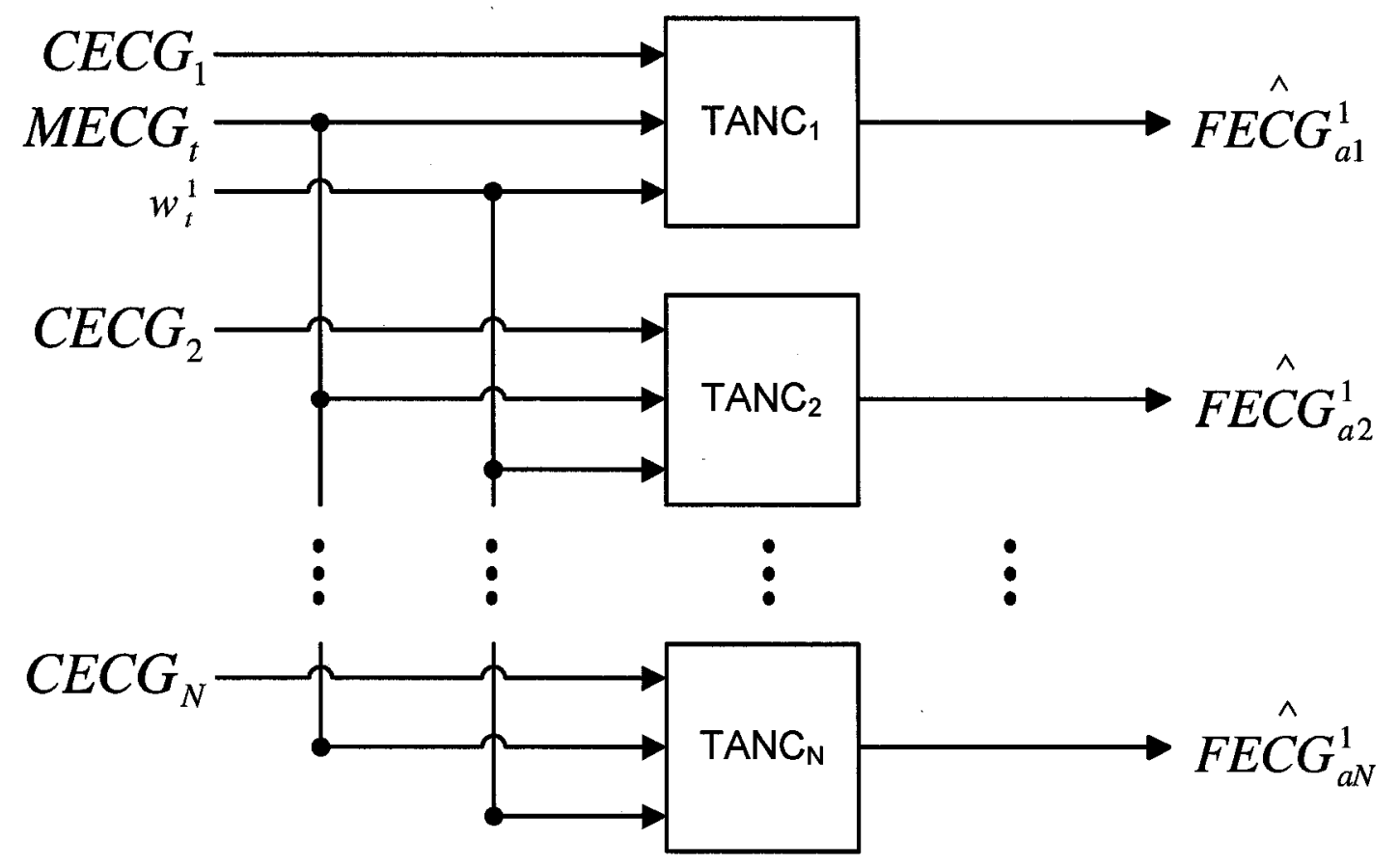

Figure 4-1 Stage 1: Multichannel TANC. Inputs $C E C G_{1}-C E C G_{N}$ are the abdominal composite signals, $M E C G_{t}$ is the thoracic MECG, and $w_{t}^{1}$ is the pulse adaptation trigger. Outputs $\hat{F E C G_{a 1}}-\hat{F E C G_{a N}}$ are $\mathbf{N}$ estimates of the desired pulse. 


\subsubsection{Stage 2: Fetal ECG Enhancement using Triggered Adaptive System Identification}

A primary concern in selecting a method for FECG extraction is to be able to process input signals that have a low SNR. To account for this, multiple abdominal channels can be acquired. Assuming that noise between channels is independent, the pulse estimate can be enhanced using TASI.

Initially, a single pulse estimate from stage 1 is selected as a template model. This selection is made by choosing the estimate with the highest $\mathrm{SNR}_{\text {est }}$ and lowest $\mathrm{PRD}_{\text {est }}$, which are defined in Section 4.4. In stage 2, each pulse estimate output of stage 1 is modeled to resemble the correlated components of the template model. Thus, uncorrelated components, i.e., random noise between channels, are reduced. This is followed by an ensemble average, which combines the results from each channel and outputs a final enhanced pulse estimate.

Figure 4-2 shows the scheme used for single-channel TASI. Note that this is identical to the TANC scheme of Figure 3-1; the differences being:

1. The output of this scheme is the direct output of the adaptive filter, which attempts to estimate the component of $M E C G_{t}$ that is correlated with $C E C G$.

2. Triggering is performed using the adapt enable constraint. The reasons for this will be discussed in Section 4.5.2.

Figure 4-2 shows the TASI scheme as applied to FECG enhancement, where $e$ represents the error of the system. Ideally, this should consist of the uncorrelated noise

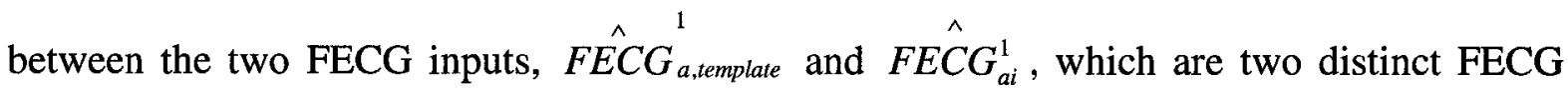


estimates output from stage 1 (see Figure 4-1). $F \hat{F C G}_{a \text {,template }}$ represents the template model $^{1}$ chosen from the outputs of stage 1 , while $F E \hat{C} G_{a i}^{1}$ represents one of the other estimates so that $i=1: \mathrm{N}$ and $i \neq$ template. $F E \hat{C} G_{a i}^{2}$ is the enhanced FECG and final output of the singlechannel TASI system.

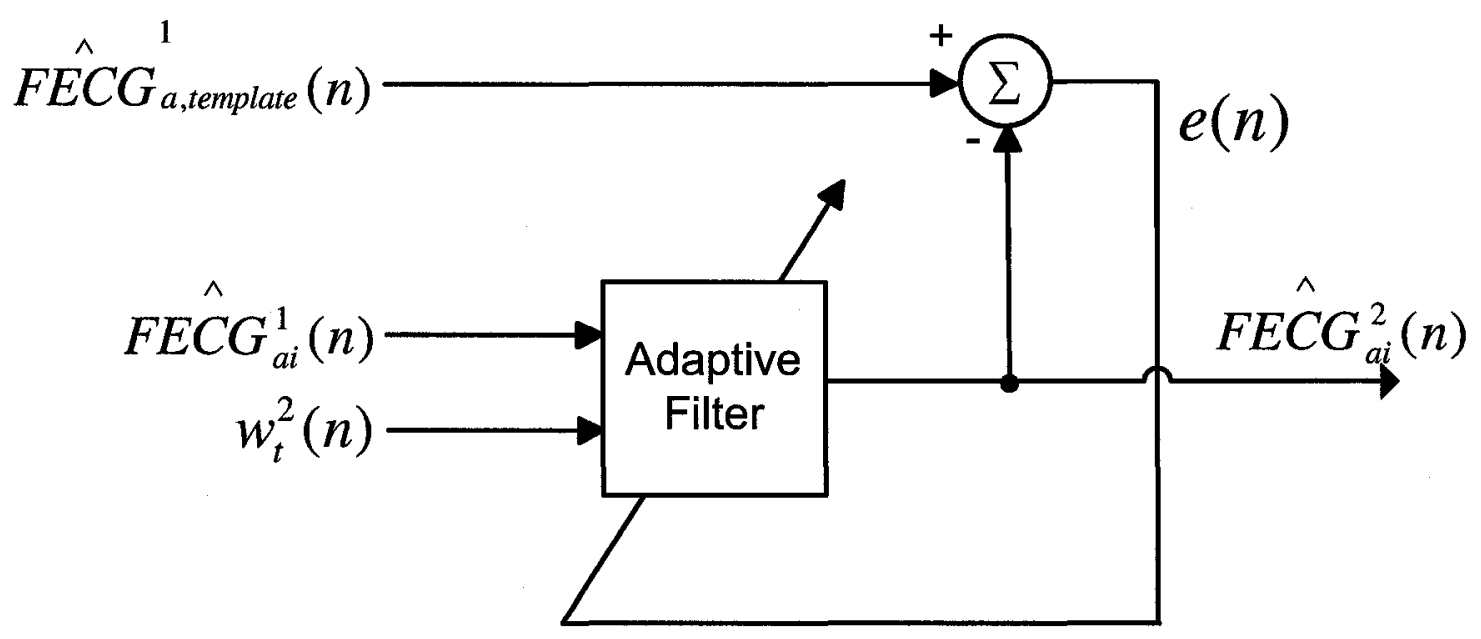

Figure 4-2 Triggered adaptive system identification applied to FECG enhancement

Stage 2, shown in Figure 4-3, employs N-1 independent TASI blocks to combine the multiple channels and enhance the desired pulse. The template model of the FECG, arbitrarily chosen in Figure 4-3 as the $\hat{F E C G_{a N}}$ output of stage 1 (see Figure 4-1), is

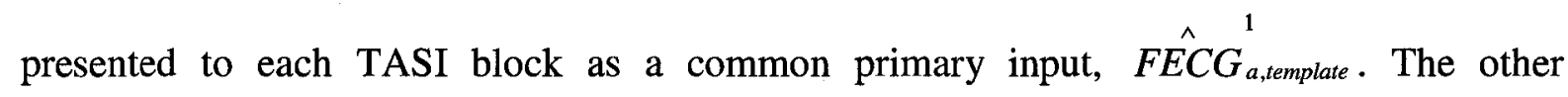
estimates from stage 1 are presented as reference inputs, $\hat{F E C G_{a 1}^{1}}-\hat{F E C G_{a N-1}}$. Under the 
assumption that the multiple channels contain uncorrelated noise, the outputs of this stage, $\hat{F E C G_{a 1}^{2}}-\hat{F E C G_{a N-1}}{ }^{2}$, represent enhanced FECG estimates with reduced noise.

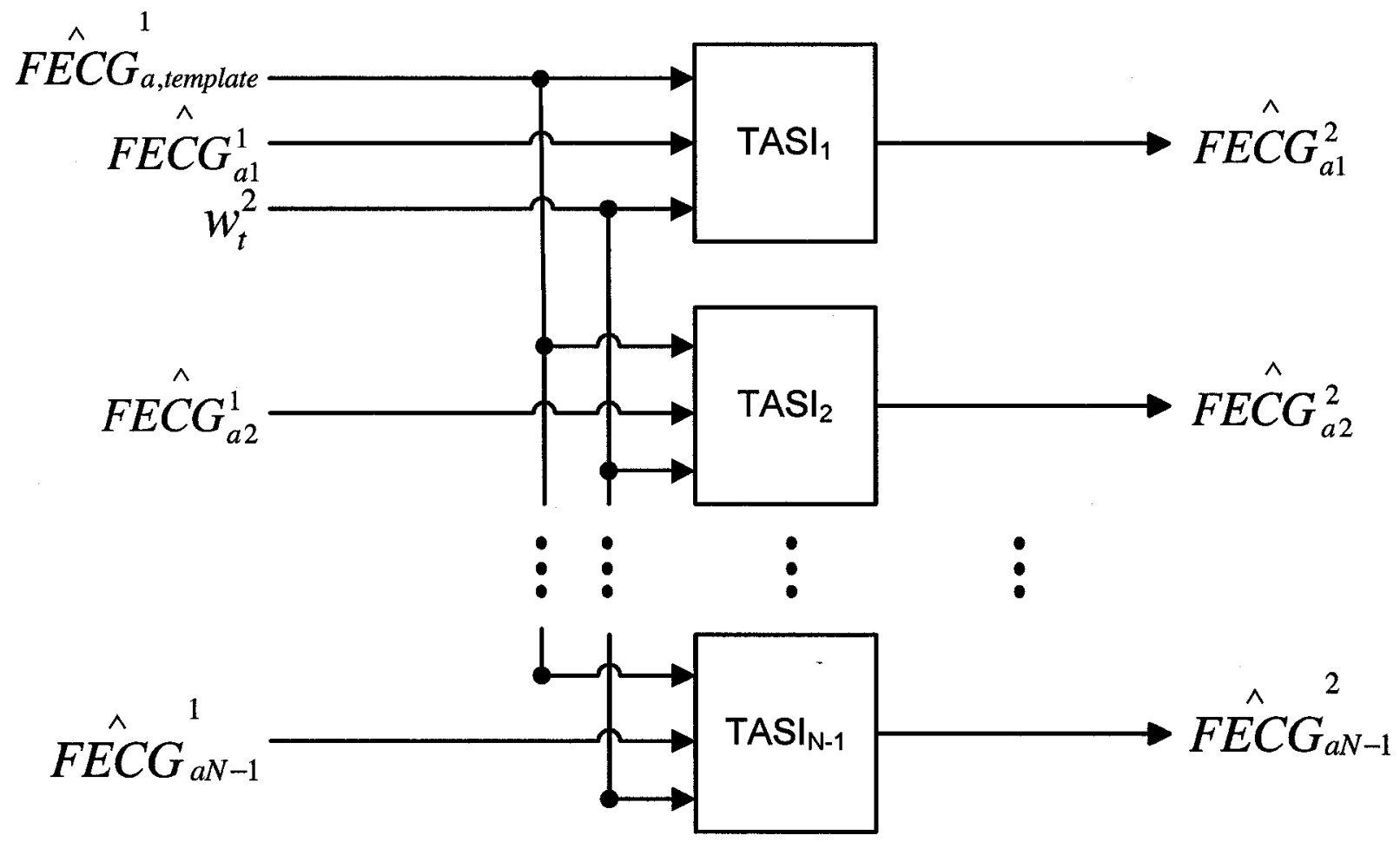

Figure 4-3 Stage 2: Multichannel TASI. Input $\hat{F E C G_{a, t e m p l a t e}}$ is the pulse estimate template model, $\hat{F E C G_{a 1}}-\hat{F E C G_{a N-1}}$ are the other pulse estimates (not including the

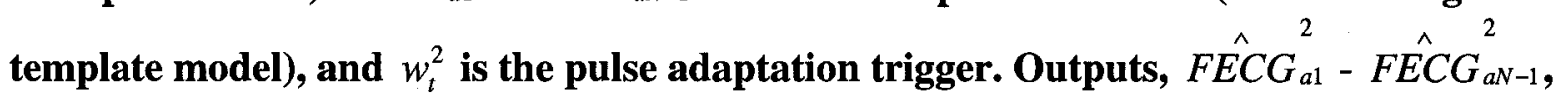
are $\mathrm{N}-1$ enhanced pulse estimates.

Following multichannel TASI, ensemble averaging is performed on its outputs, $\hat{F E C G_{a 1}}-\hat{F E C G_{a N-1}}$, along with the template model input, $\hat{F E C G_{a, t e m p l a t e}}$. This final step, in combination with stage 1 and stage 2, is depicted in Figure 4-4. 


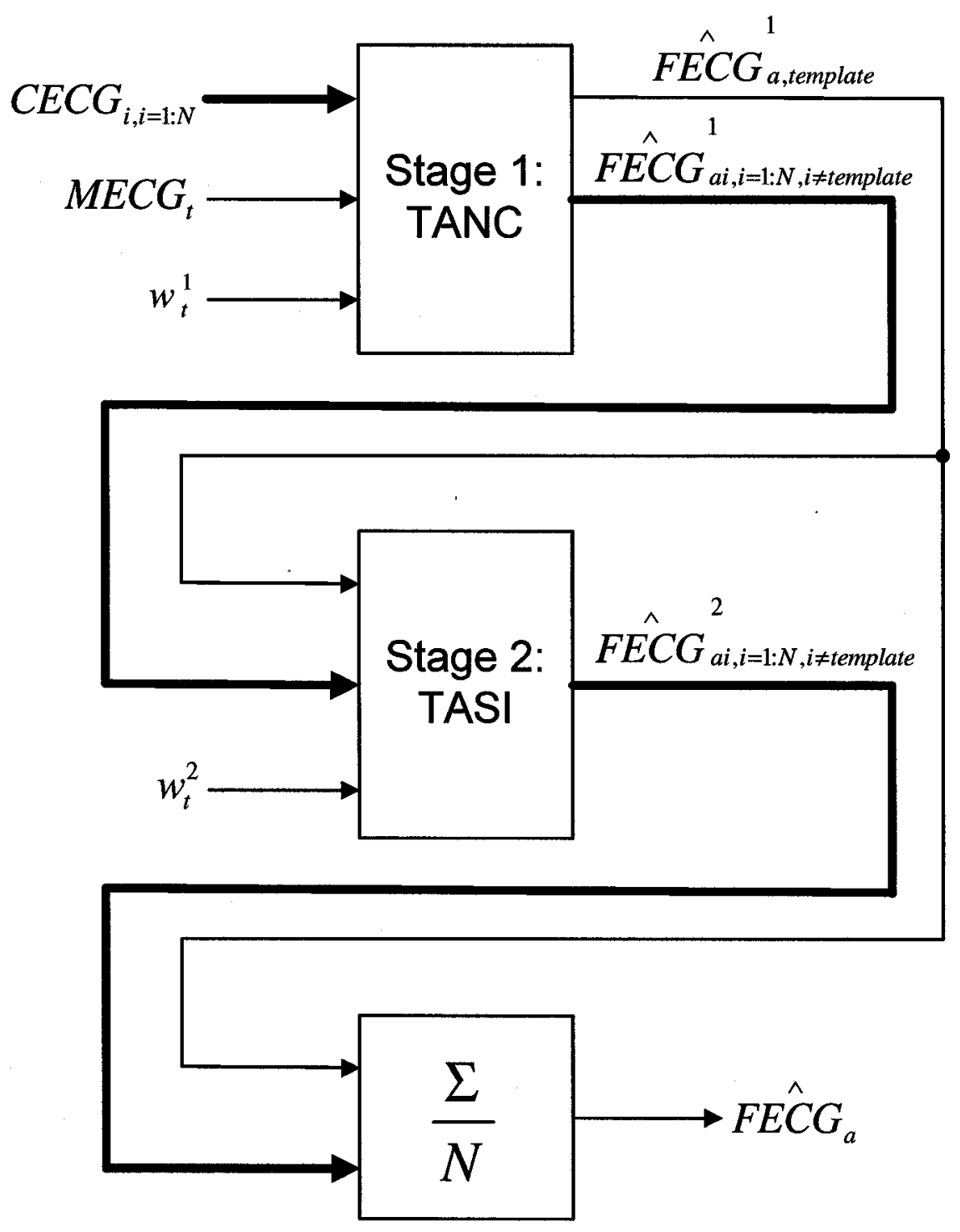

Figure 4-4 Two-stage multichannel signal extraction. Inputs to stage 1: $C E C G_{i, i=1: N}$ are the abdominal composite signals, $M E C G_{t}$ is the thoracic MECG, and $w_{t}^{1}$

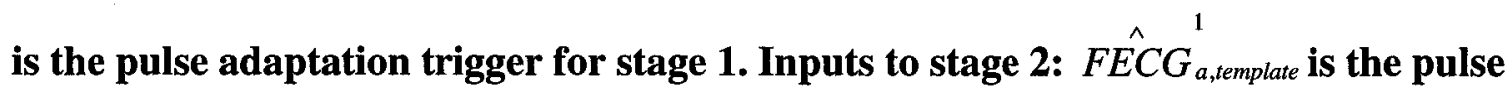
estimate template model, $\hat{F E C G_{a i, i=1:, i \neq t e m p l a t e}}$ are the other pulse estimates and $w_{t}^{2}$ is the pulse adaptation trigger for stage 2 . The final output, $F \hat{E C} G_{a}$, is the ensemble average of the enhanced pulse estimates, $F E C G_{a i, i=1: N, i \neq t e m p l a t e}$, from stage 2 and the template

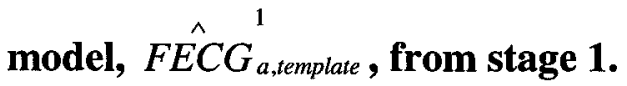




\subsection{Experimental Methods}

The data used to test the two-stage multichannel FECG extraction system were collected from a non-pregnant subject, using an external electrical SP to represent the FECG. This study has been reviewed and received ethics clearance though the Carleton University Research Ethics Committee in accordance to the Tri-Council Policy Statement for Ethical Conduct for Research Involving Humans (refer to Appendix A for consent form). An SP was used for this study to provide control of the amplitude and timing of the pulses by adjusting the pulse strength and rate of delivery, respectively. A current pulse was applied externally to the back of the subject and simultaneously acquired from multiple channels on the subject's front, as illustrated in Figure 4-5. The pulse is allowed to propagate through the abdomen, experiencing tissue filter effects and additively mixing with the subject's ECG and other interference sources; similar to processes experienced by an FECG before it is noninvasively acquired.

The stimulus was a rectangular pulse delivered to the subject's back using a stimulator (Grass S48, Astro-Med - Rhode Island, U.S.A.) with a constant-voltage isolation unit (Grass SIU5, Astro-Med - Rhode Island, U.S.A.), with varying amplitudes between 10$20 \mathrm{~V}$ (current range: $0.1-1.5 \mathrm{~mA}$ ) at a rate of 2 pulses per second (i.e. $120 \mathrm{bpm}$, which is in the range of a typical fetal heart rate), and duration of $20 \mathrm{~ms}$ per pulse. The duration of the pulse was chosen specifically so that the duration of acquired pulse on the subject's front was similar to the duration of an FECG. The pulse was delivered to the subject's lower back using an $\mathrm{Ag}-\mathrm{AgCl}$ electrode pair (Red-Dot 2237, 3M - Ontario, Canada), off-axis from the spine to avoid delivery directly to bone matter. 

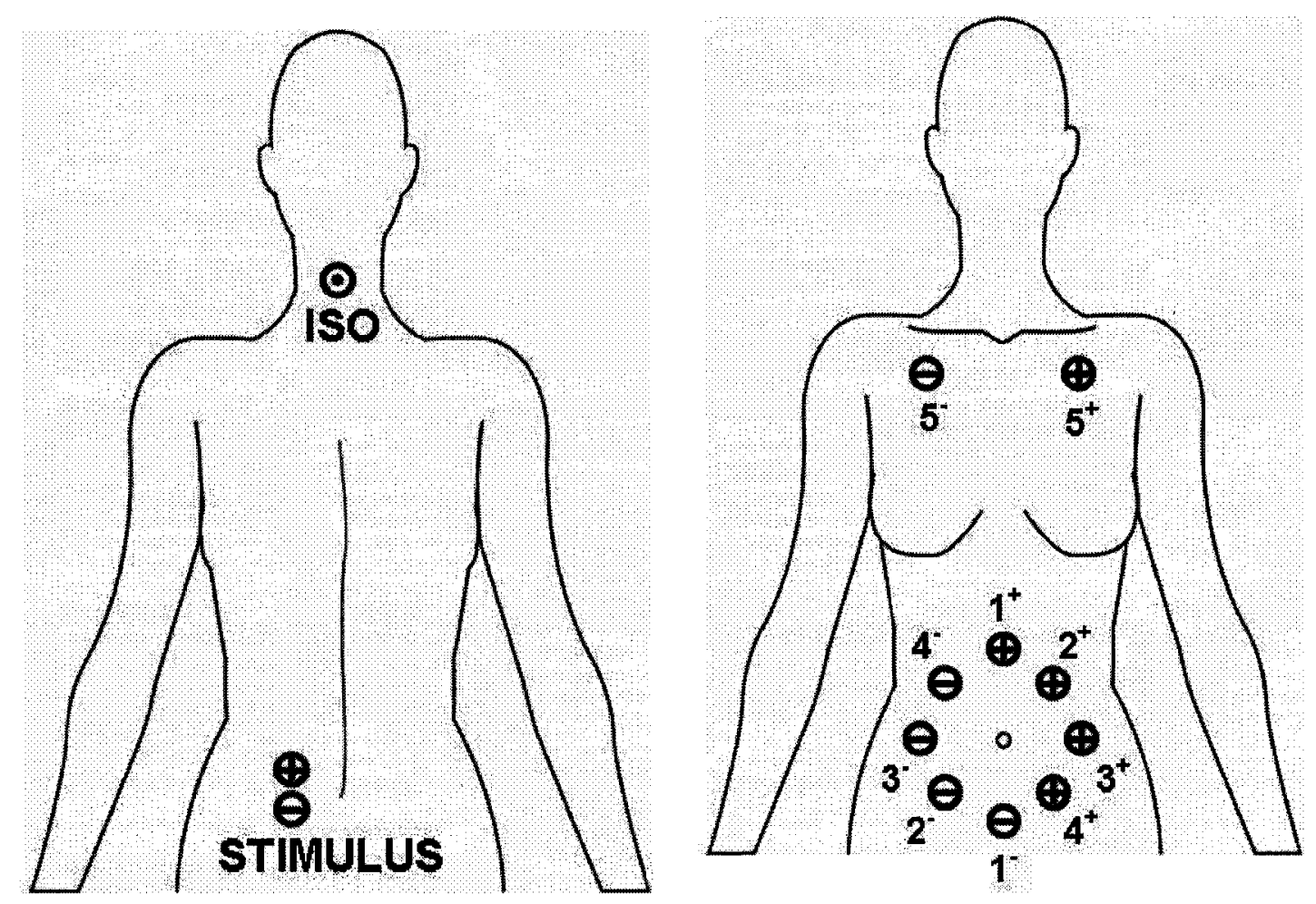

Figure 4-5 Data acquisition and stimulus delivery electrode placements on front and back of subject

Five minutes of data from multiple recording sites were collected from the subject's front using $\mathrm{Ag}-\mathrm{AgCl}$ surface electrodes (Red-Dot 2237, 3M - Ontario, Canada). A circular (radius $=7 \mathrm{~cm}$ ) electrode placement configuration was used, shown in Figure 4-5. This consisted of four channels of abdominal composite data. One channel of thoracic ECG data were collected, and the back of the neck was used as a common ground reference. In addition, the external stimulus signal was collected to provide temporal locations of the applied SP.

Data were differentially amplified using a high gain AC amplifier (Model 15A54, Grass Telefactor - Rhode Island, U.S.A.), with a gain of 1000 and bandwidth of 1-300 Hz. Signals were sampled at $1000 \mathrm{~Hz}$ using a 12-bit analog-to-digital converter board (PCI71 
6071E, National Instruments - Quebec, Canada). After data acquisition, the signals were lowpass filtered with a cutoff frequency of $100 \mathrm{~Hz}$ and downsampled to a sampling frequency of $250 \mathrm{~Hz}$ (offline processing). Power-line interference was removed adaptively by including in the data collection a channel only containing the $60 \mathrm{~Hz}$ interference (i.e., a bioamplifier with no input connections), which was used as the primary channel for an adaptive noise canceller (similar to the procedure used in Section 3.3 to adaptively remove $60 \mathrm{~Hz}$ interference).

Figures 4-6 - 4-9 display segments of the acquired data from each channel with SP amplitudes of $10,12,14$ and $20 \mathrm{~V}$, respectively. Abdominal channel 1 of each figure most prominently shows the applied stimulus, while channel 3 contains the least SP amplitudes. We suspect the reason for this can be deduced from the electrode configurations as seen in Figure 4-5. Channel 1 is aligned with the SP delivery electrodes, and thus provides a maximum potential difference between the positive and negative electrode sites. Channel 3 , on the other hand, is perpendicular to the SP delivery electrodes and appropriately displays a smaller potential difference between the acquisition electrodes. 


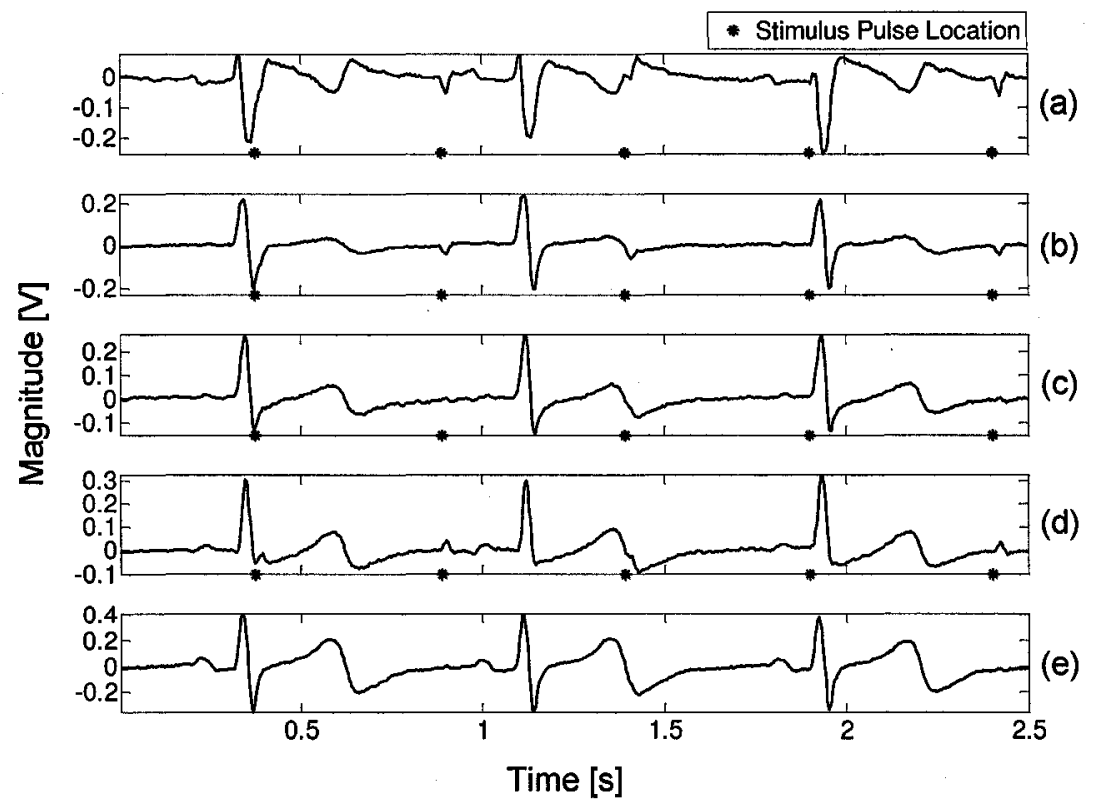

Figure 4-6 Acquired ECG data with SP amplitude of $10 \mathrm{~V}$ : (a)-(d) Abdominal channels 1-4, respectively. (e) Thoracic channel 5

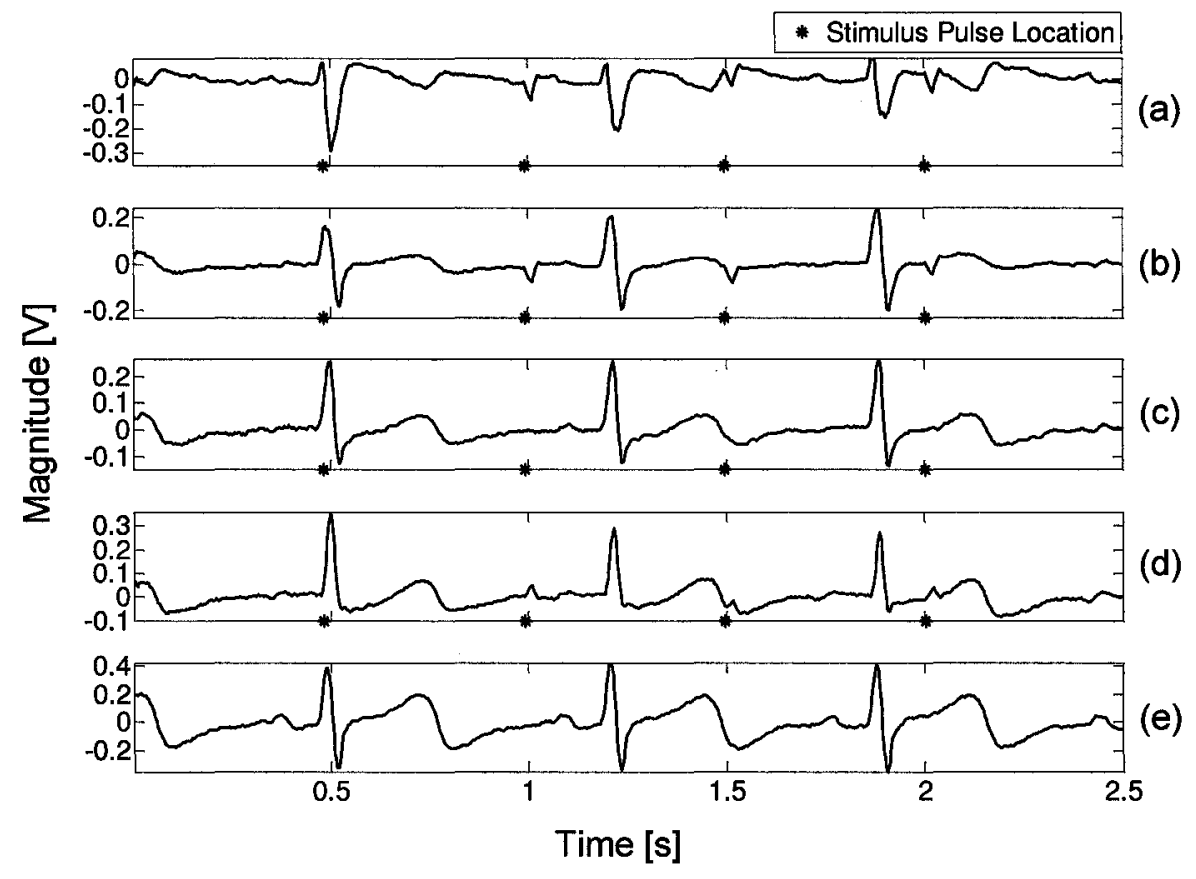

Figure 4-7 Acquired ECG data with SP amplitude of $12 \mathrm{~V}$ : (a)-(d) Abdominal channels 1-4, respectively. (e) Thoracic channel 5 


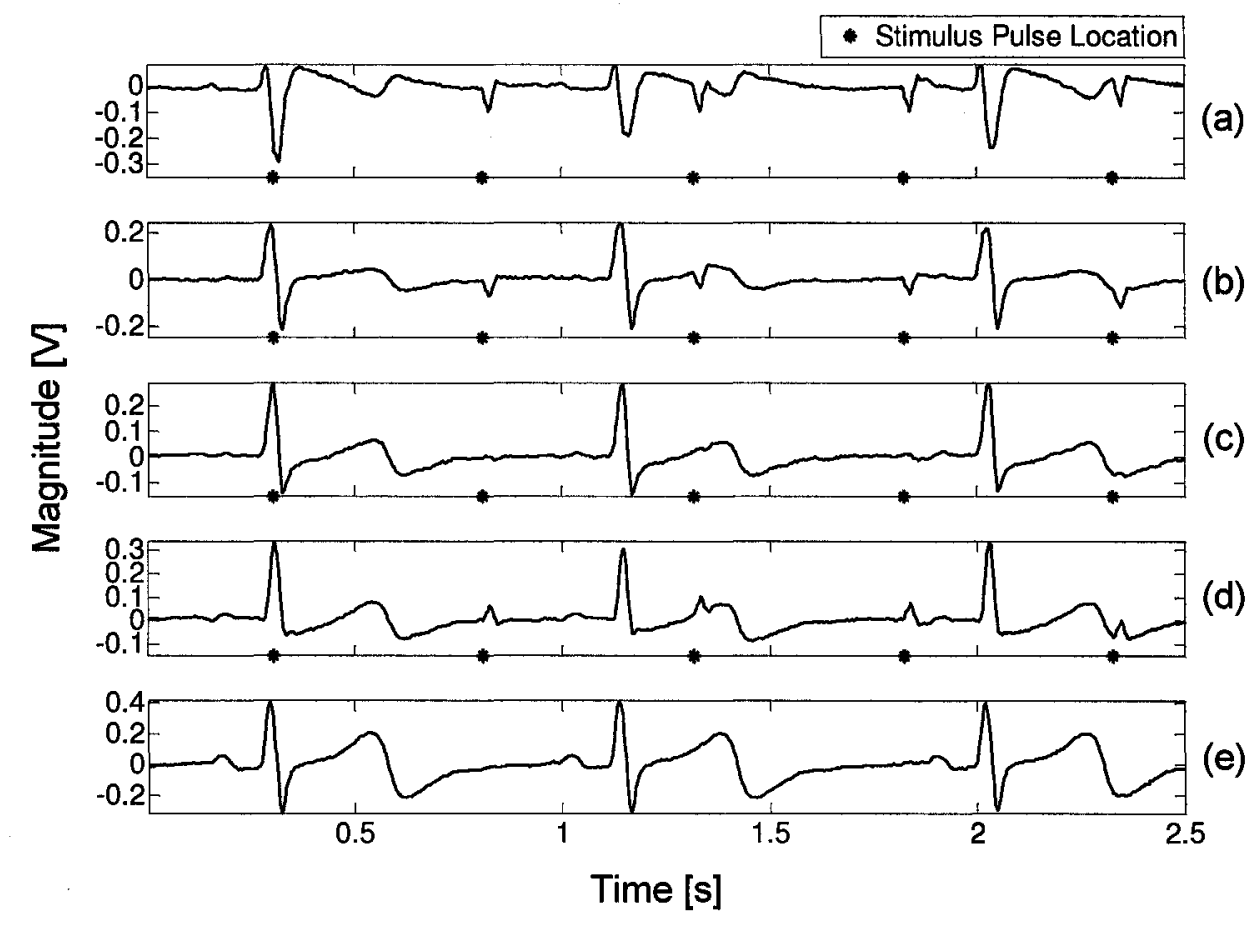

Figure 4-8 Acquired ECG data with SP amplitude of 14 V: (a)-(d) Abdominal channels $1-4$, respectively. (e) Thoracic channel 5

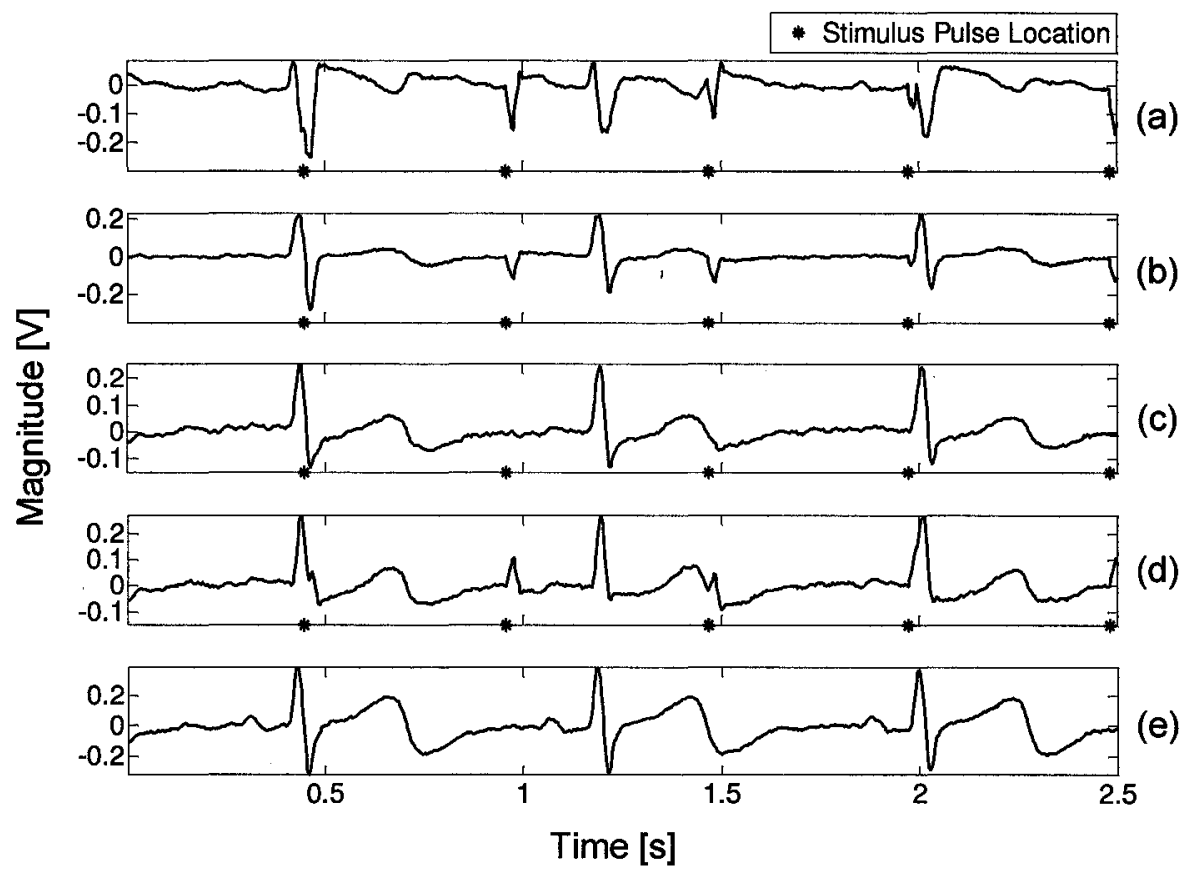

Figure 4-9 Acquired ECG data with SP amplitude of 20 V: (a)-(d) Abdominal channels 1-4, respectively. (e) Thoracic channel 5 


\subsection{Performance Measures}

For this study, there is no ideal pulse with which to compare the system's estimated output. Thus, conventional performance measures (e.g., PRD) cannot be applied. To analyze the results, we define two secondary performance measures.

\subsubsection{SNR $\mathrm{SN}_{\mathrm{est}}$}

The SNR estimate, $S N R_{\text {est }}$, is defined here as the ratio of the estimated SP power to the estimated noise power in the data. The noise power is considered only during periods where the SP does not occur. To determine the periods during which the SP occurs, we begin by taking a time-average of the SP using Equation 4.1. By performing time-averaging we can effectively ignore the effects of the additive noise components that are present during the SP. This allows us to consider $V$ as the average SP shape in the acquired data, shown in Figure 4-10 for channel 2. Assuming that the noise power is the same whether an SP occurred or not, and that the SP is passed undistorted through the TANC and TASI systems, this shape is the expected desired pulse. A visual analysis of Figure 4-10 reveals that the SP has a duration of $0.22 \mathrm{~s}$. The time-averaged SP duration is similar across channels.

$$
V(j)=\frac{1}{M} \sum_{i=1}^{M} A_{S P, i j} \quad \text { for } j=1: L
$$

where $\quad A_{S P, i j}:$ Amplitude of $i^{\text {th }} \mathrm{SP}$ at sample $j$,

$M$ : Total number of SPs,

$L:$ Number of samples per SP. 


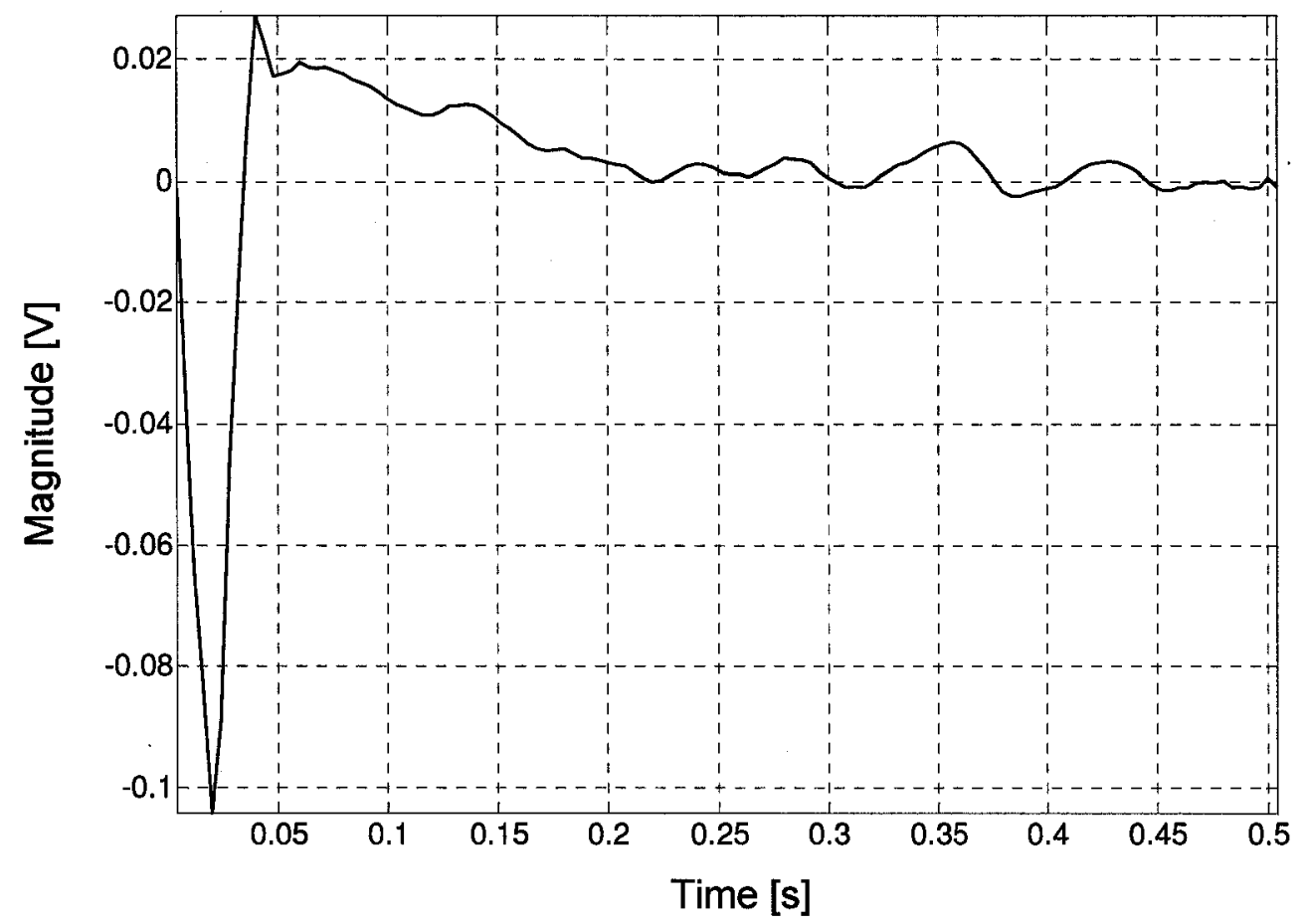

Figure 4-10 Time-average of the SP

We can now calculate the $\mathrm{SNR}_{\text {est }}$ by defining the periods where the SP occurs as the desired signal and all other regions as noise, which includes the subject's ECG and random noise. This is not a completely accurate measure of the SNR, only an estimate, since remnants of the subject's ECG and random noise overlap with the SP; however, this is an unavoidable consequence of not having an ideal SP. Equation 4.2 shows the calculation of the $\mathrm{SNR}_{\mathrm{est}}$, and reflects that the signal power contains additive noise power as well. In this equation, the signal data, $x$, are created by zeroing samples during which the SP does not occur. Conversely, the noise data, $n$, is created by zeroing samples during which the SP does occur. 


$$
S N R_{e s t}=\frac{\frac{1}{N_{x}} \sum_{i=1}^{N_{x}}|x+n|^{2}}{\frac{1}{N_{n}} \sum_{i=1}^{N_{n}}|n|^{2}},
$$

where $x$ : Signal data in Volts,

$N_{x}$ : Signal data length,

$n$ : Noise data in Volts,

$N_{n}$ : Noise data length.

In the case of a high amplitude applied SP, it is reasonable to assume that the noise power is much smaller than the signal power, thus bringing the $\mathrm{SNR}_{\text {est }}$ closer to the actual SNR. Under this assumption, Equation 4.2 can be reduced to Equation 4.3 . It is important to note that the $\mathrm{SNR}_{\text {est }}$ is not a measure of the accuracy of the extracted pulse. It is only used as a measure of the degree of attenuation of the subject's ECG and random noise. Even in the case where the noise power is not small compared to the signal power, as in Equation 4.2, the $\mathrm{SNR}_{\text {est }}$ still increases when the actual SNR increases; therefore, it still provides a measure of the attenuation of the interferences.

$$
S N R_{e s t} \approx \frac{\frac{1}{N_{x}} \sum_{i=1}^{N_{x}}|x|^{2}}{\frac{1}{N_{n}} \sum_{i=1}^{N_{n}}|n|^{2}},
$$

The $\mathrm{SNR}_{\mathrm{est}}$ performance measure is calculated based on the last $60 \mathrm{~s}$ of data (15000 samples using $f_{s}=250 \mathrm{~Hz}$ ). This ensures that the adaptive filter, which has been given input data with $300 \mathrm{~s}$ duration (75000 samples), has fully converged. Convergence was verified visually. 


\subsubsection{PRD est}

The PRD estimate, $P R D_{\text {est }}$, performance measure defined here does not make use of an ideal pulse. Instead, the time-averaged SP, given by Equation 4.1, of the acquired data is defined as the best SP estimate. Although slight variations between individual pulses will be exhibited in the acquired data due to fluid and organ movement in the abdomen during data acquisition, time-averaging is expected to decrease this variability, as well as additive interferences, and provide a reasonable estimate of the desired SP.

The $\mathrm{PRD}_{\text {est }}$ is calculated using Equation 3.16, with the ideal signal being the timeaveraged estimate provided per channel. For each channel, the $\mathrm{PRD}_{\mathrm{est}}$ values are calculated separately per pulse, and the results averaged. The reason for doing this is that there exists only a single SP after time-averaging, thus each estimated SP must be compared separately to it. Similar to the $\mathrm{SNR}_{\text {est }}$ measure, the PRD est measure is calculated based on the last $60 \mathrm{~s}$ of data (15000 samples using $\left.f_{s}=250 \mathrm{~Hz}\right)$.

\subsection{Results}

In this section, we examine the results of the two-stage extraction and enhancement scheme detailed in Section 4.2.

\subsubsection{Stage 1 Results}

Simulations for stage 1 (i.e., ANC) were initially run using no triggering to investigate an optimal filter order. The RLS adaptive algorithm with adaptive memory (i.e., time-varying forgetting factor $\lambda$ ) was used in our simulations with an FIR filter. An initial $\lambda$ 
of 0.999 with time-varying values between 0.999 and 0.9999 (increments of 0.0001 ) was used. Figure 4-11 displays the calculated SNR $_{\text {est }}$ values of the estimated SP for varying filter orders using an applied SP amplitude of $20 \mathrm{~V}$. Note that the power of the extracted SP is now greater than that of the subject's ECG, which has been attenuated. The SNR est $_{\text {initially }}$ increases rapidly (especially channels 1 and 2), approaching and finally settling at an optimal value before gradually decreasing at the higher filter orders. This analysis is similar for the $P R D_{\text {est }}$ results shown in Figure 4-12, noting that a smaller $P_{R D}$ est indicates better results. A filter order of 256 was chosen as empirically optimal between all four channels.

Figures 4-6 - 4-9 clearly show an increase in the amplitude of the acquired SP as the applied SP amplitude is increased. However, due to the orthogonal relationship between the stimulus electrode configuration and the data acquisition electrode configuration, the stimulus pulse is not visible in channel 3. The effect of this phenomenon is clearly evident in Figures 4-11 and 4-12. In Figure 4-11, the SNR $_{\text {est }}$ values associated with channel 3 across all filter orders are small, and the power of the SP does not exceed that of the noise even after ANC. Similarly in Figure 4-12, only the PRD $_{\text {est }}$ values associated with channel 3 exceed 100 $\%$, which implies that the noise power is greater than the signal power. 


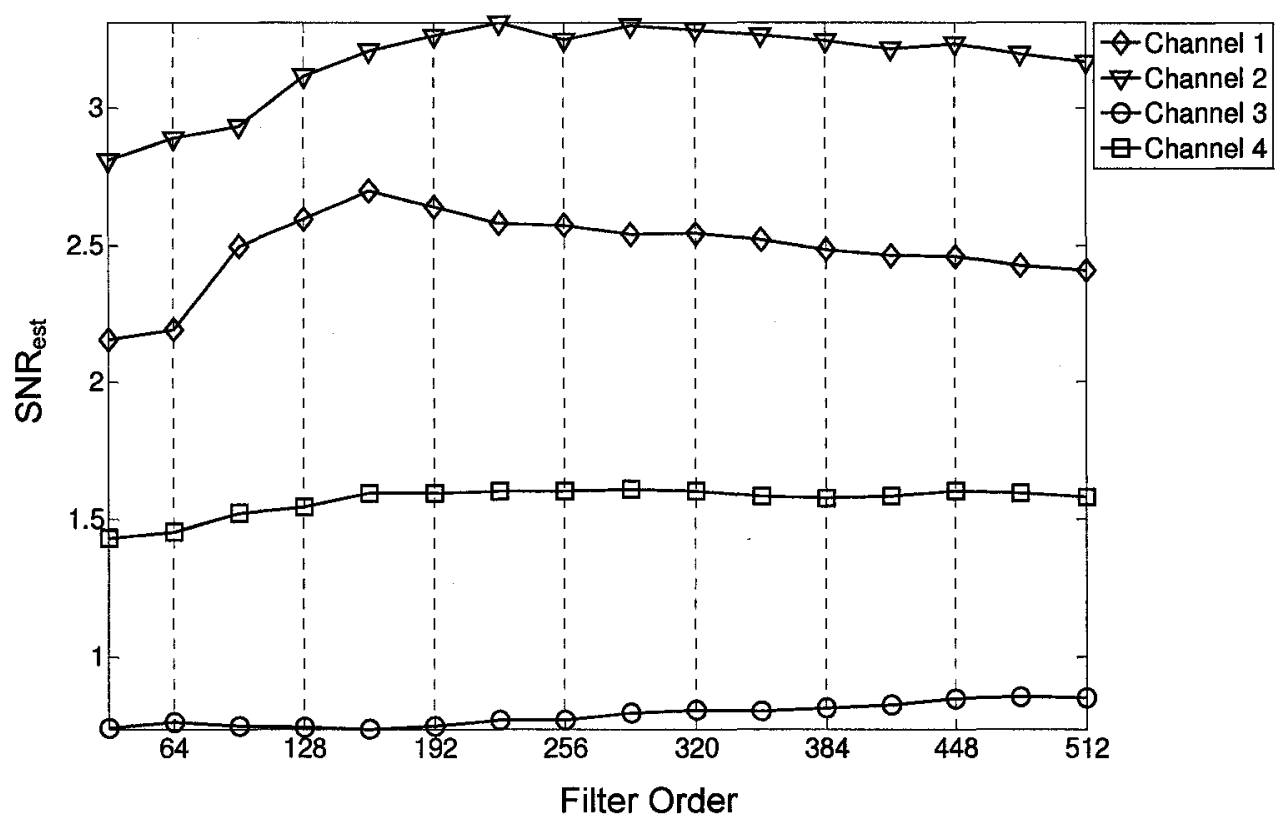

Figure 4-11 SNR $_{\text {est }}$ of abdominal channels after ANC for various filter orders using an applied SP amplitude of $20 \mathrm{~V}$ and no triggering

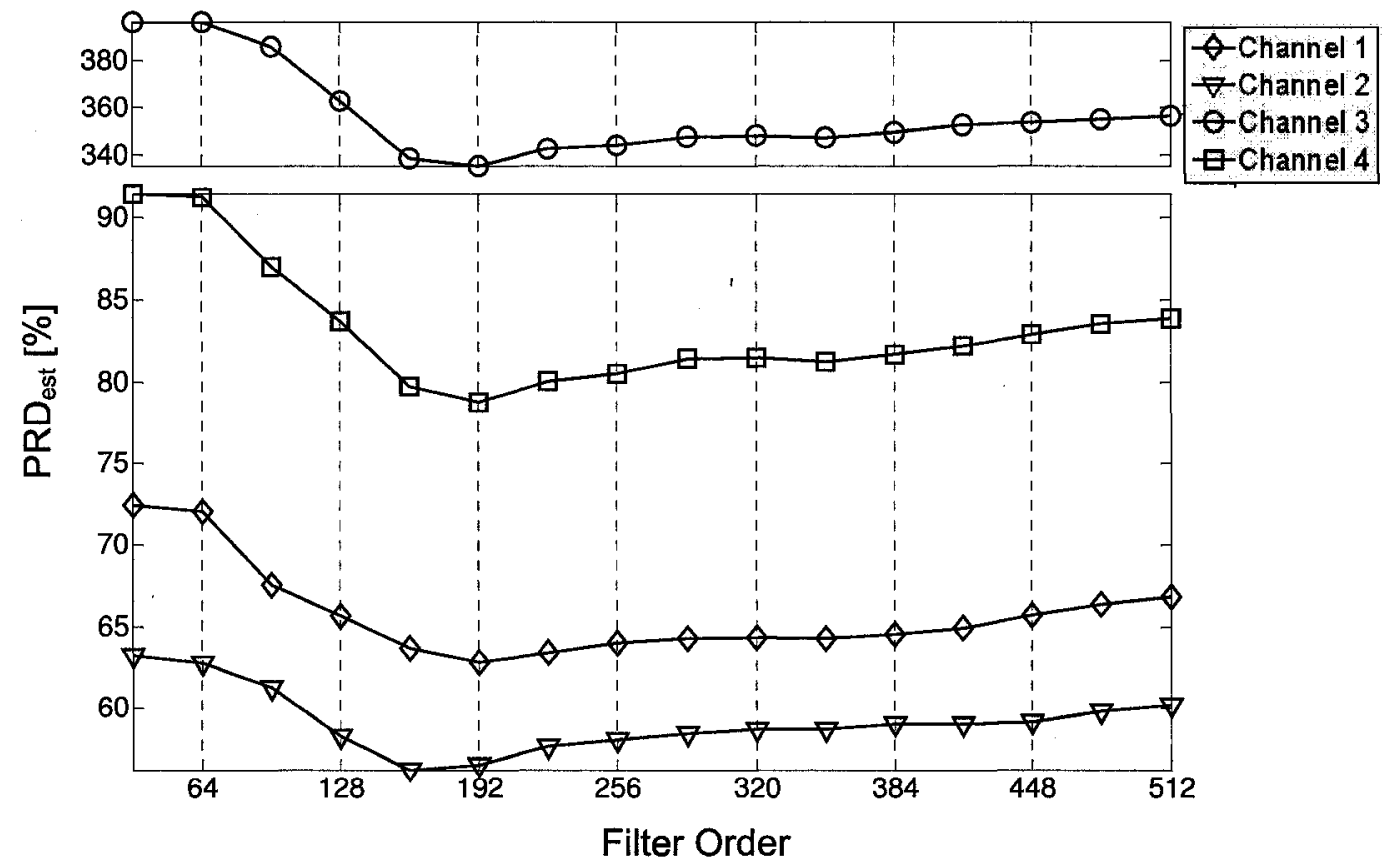

Figure 4-12 PRD $_{\text {est }}$ of abdominal channels after ANC for various filter orders using an applied SP amplitude of $20 \mathrm{~V}$ and no triggering

80 
Using the $20 \mathrm{~V}$ applied SP amplitude, further simulations were run using TANC, now incorporating triggering, and using the RLS adaptive algorithm with adaptive memory in an FIR filter. The filter used had an order of 256 , and an initial $\lambda$ of 0.999 with time-varying values between 0.999 and 0.9999 (increments of 0.0001 ). Triggering was used with on/off transitions as well as weighted linear transition triggering with the adapt-disable constraint enforced and a minimum trigger weight of 0.05 . This is illustrated in Figure $4-13$, where it can be seen that adaptation is disabled during the SP using triggering with on/off and linear transitions, while adaptation is always enabled in the case of no triggering.

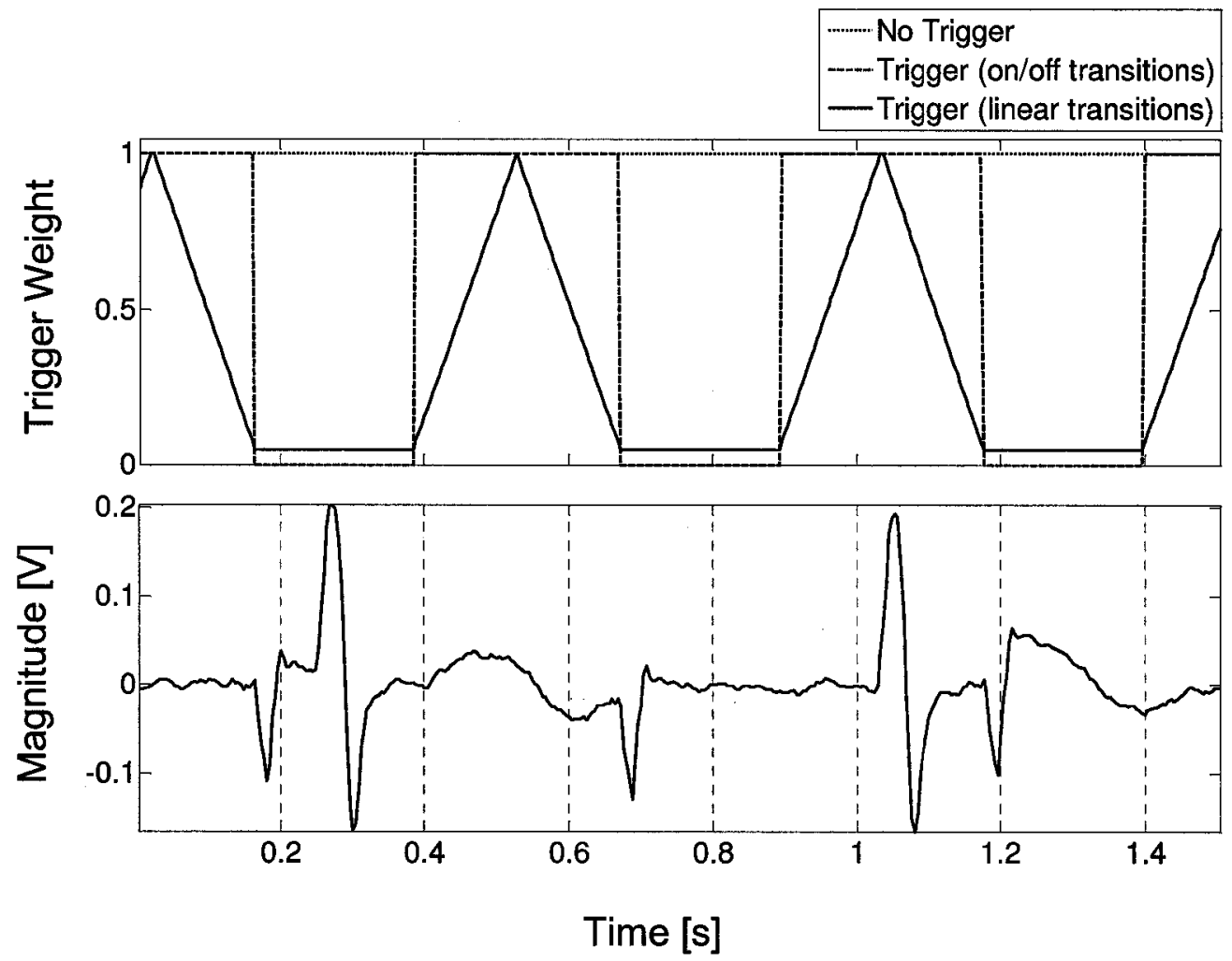

Figure 4-13 Triggering modes used for TANC 
The results using TANC with on/off transitions are better than that provided without triggering. This is evident through the higher $\mathrm{SNR}_{\text {est }}$ seen in Figure 4-14, and lower $\mathrm{PRD}_{\mathrm{est}}$ in Figure 4-15. Although the $\mathrm{SNR}_{\text {est }}$ does not give a measure of the accuracy of the extracted SP, we can state that the use of triggering provides a better attenuation of the subject's ECG. It would be reasonable, based on the simulations detailed in Chapter 3, to expect that the accuracy of the extracted SP is indeed better with triggering, especially using linear transitions with the adapt-disable constraint enforced. The $\mathrm{PRD}_{\text {est }}$ measure of Figure 4-15, on the other hand, does indicate higher accuracy with respect to the ensemble averaged SP of the acquired data.

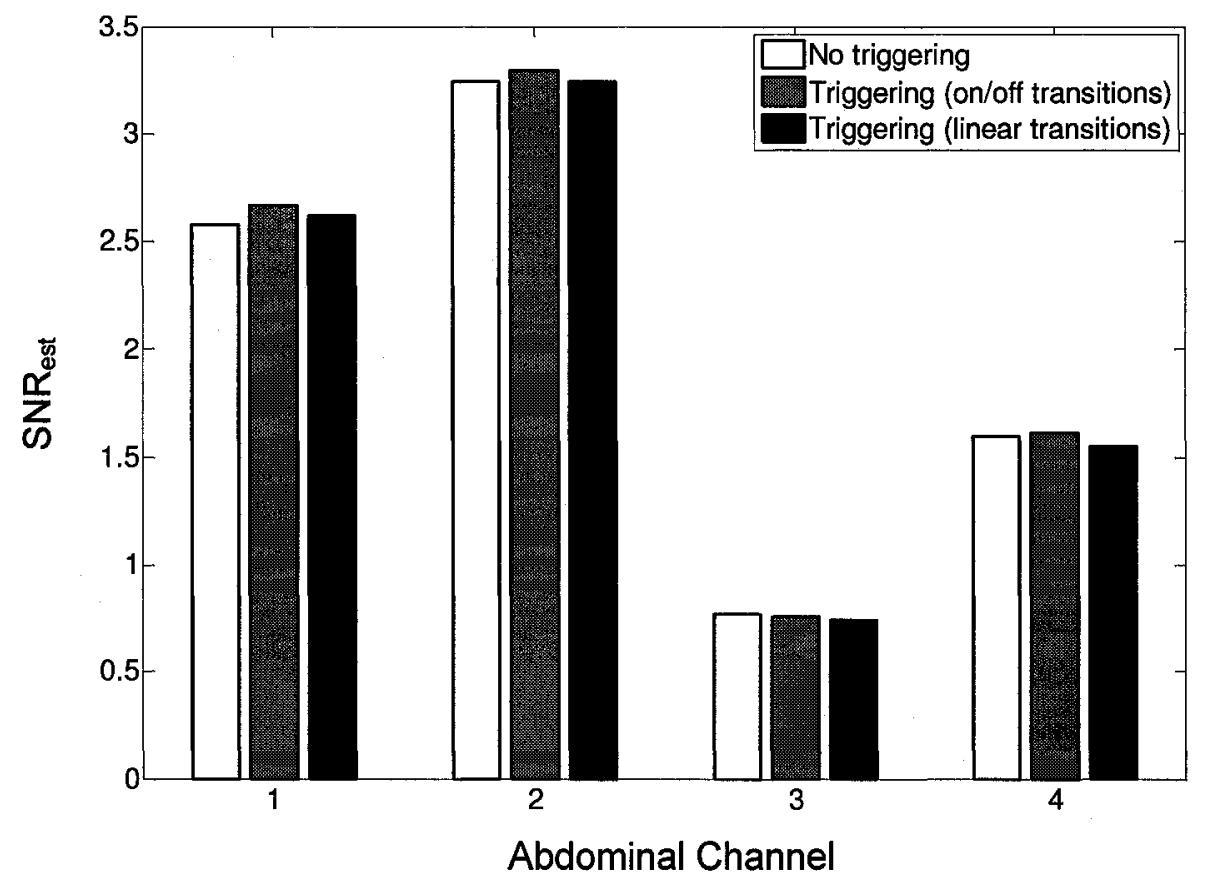

Figure 4-14 SNR $_{\text {est }}$ of abdominal channels after TANC for various triggering modes and an applied SP amplitude of $20 \mathrm{~V}$ 


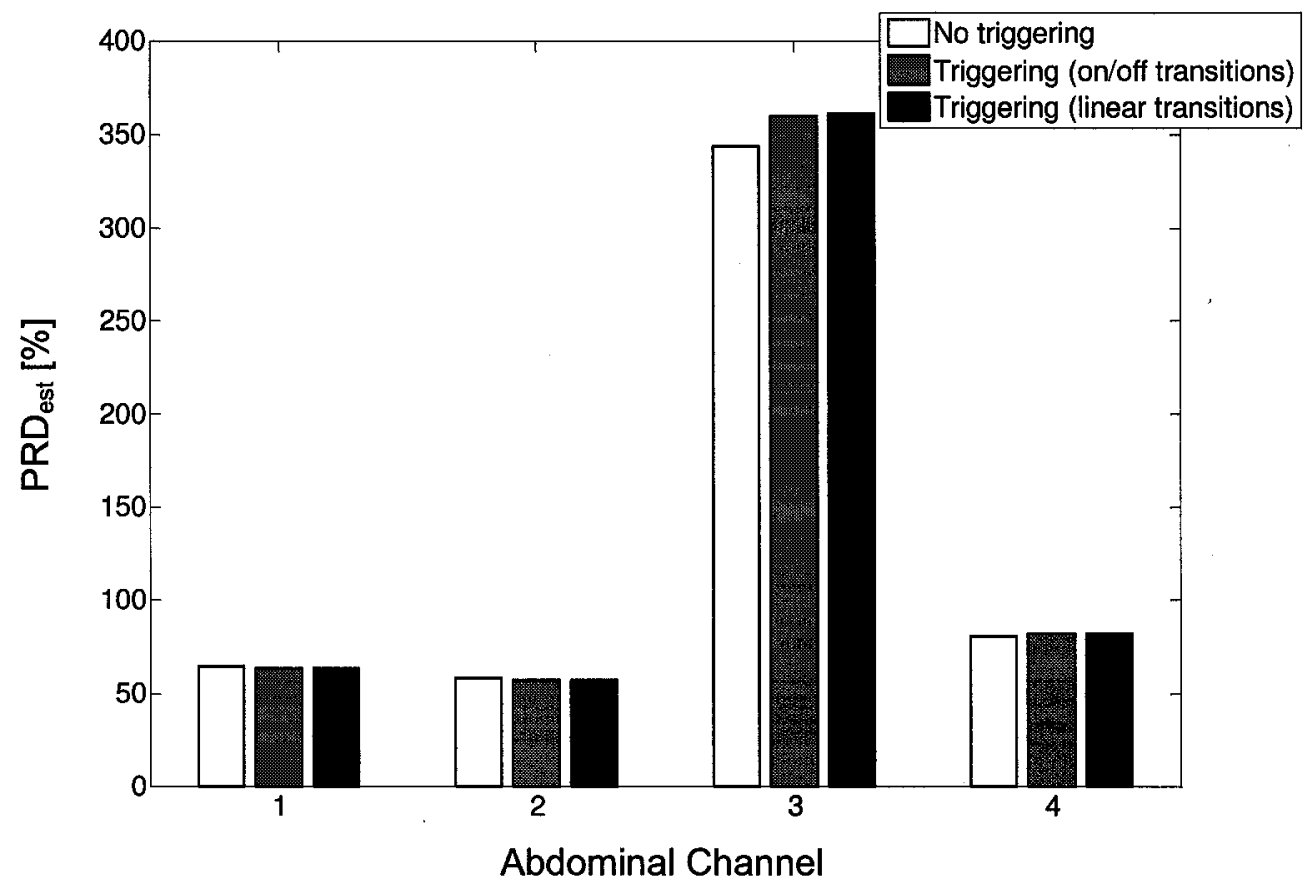

Figure 4-15 PRD $_{\text {est }}$ of abdominal channels after TANC for various triggering modes and an applied SP amplitude of $20 \mathrm{~V}$

Figure 4-16 displays a segment of the data following TANC using various triggering modes to give a visual representation extracted SP. Although the SP is now the dominating signal, remnants of the subject's ECG remain at $0.25 \mathrm{~s}$ and $1.05 \mathrm{~s}$. This is a reminder of the difficulty experienced by adaptive filters when presented with low $\mathrm{SNR}_{\mathrm{est}}$ inputs. It is not visually apparent whether the use of triggering outperforms classic ANC in terms of accuracy (i.e., less distortion of the SP estimate), and we cannot measure the accuracy due to the absence of an ideal SP. Given the low PRDs (indicating high accuracy) presented in Chapter 3 using synthetic ECG data, any improvement in accuracy provided by triggering cannot be expected to be seen visually. The advantage of the expected higher accuracy provided by 
triggering lies not in a subjective analysis, but an objective one in which specific time intervals and relative amplitudes within the ECG component waves are clinically important.

(a) No Triggering

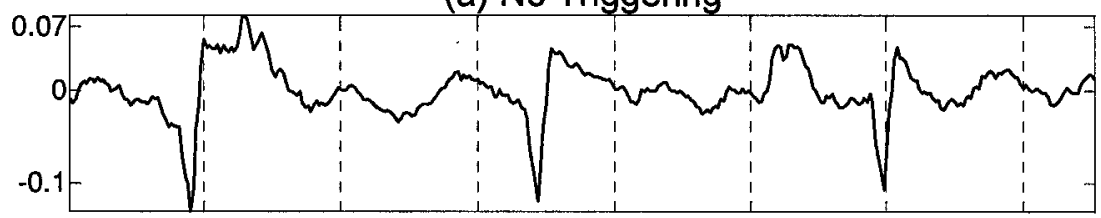

(b) Triggering (on/off transitions)

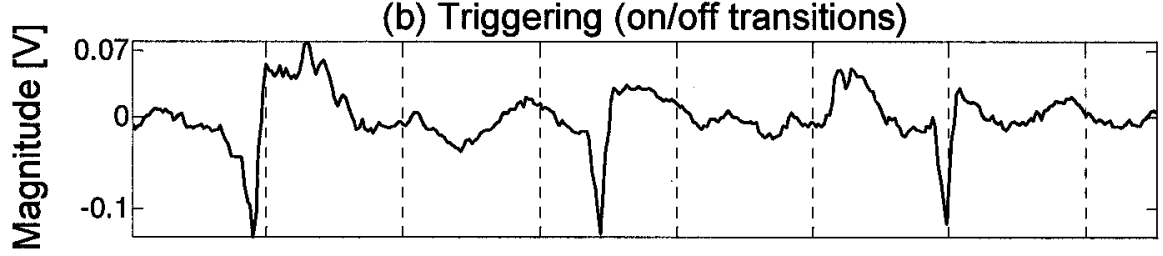

(c) Triggering (linear transitions)

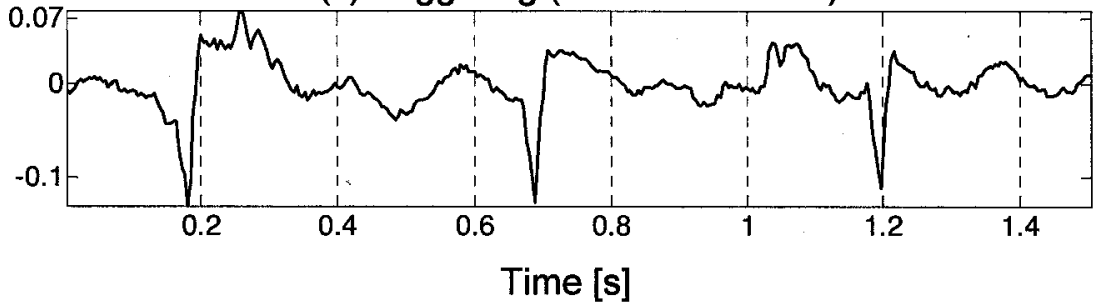

Figure 4-16 Estimated SP after TANC using channel 2 for various triggering modes with an applied SP amplitude of $20 \mathrm{~V}$

\subsubsection{Effect of lowering the SNR of the acquired data}

Data were initially collected from the test subject using various applied SP amplitudes. Thus far, the results shown for stage 1 used the highest applied amplitude of 20 V. The SP amplitudes shown in Figures 4-6 - 4-8 for 10, 12 and $14 \mathrm{~V}$ applied SP amplitudes, respectively, are quite smaller. Further simulations were run using the same filter specifications as that of Section 4.5.1 (i.e., RLS adaptive algorithm, FIR filter order: 256, initial $\lambda$ : 0.999 with time-varying values between 0.999 and 0.9999 in increments of 0.0001 ). Triggering was used with on/off transitions as well as weighted linear transition triggering with the adapt-disable constraint enforced and a minimum trigger weight of 0.05 . These 
results are compared once again to classic ANC with no triggering. Figures 4-17 - 4-22 show the $\mathrm{SNR}_{\text {est }}$ and $\mathrm{PRD}_{\text {est }}$ results of the outputs of stage 1 using applied SP amplitudes of 14,12 and $10 \mathrm{~V}$. These figures will be referred to in Section 4.5.2.1.

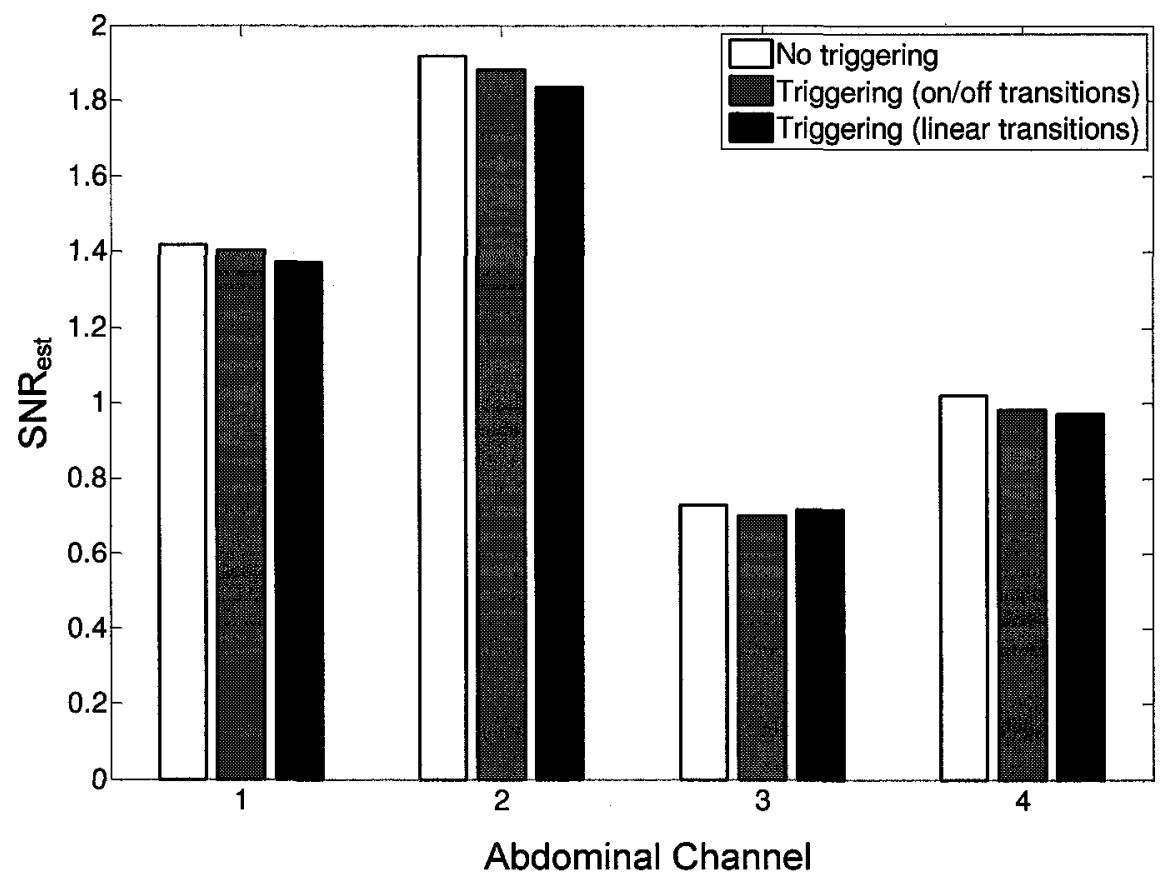

Figure 4-17 SNR $_{\text {est }}$ of abdominal channels after TANC for various triggering modes with an applied SP amplitude of $14 \mathrm{~V}$ 


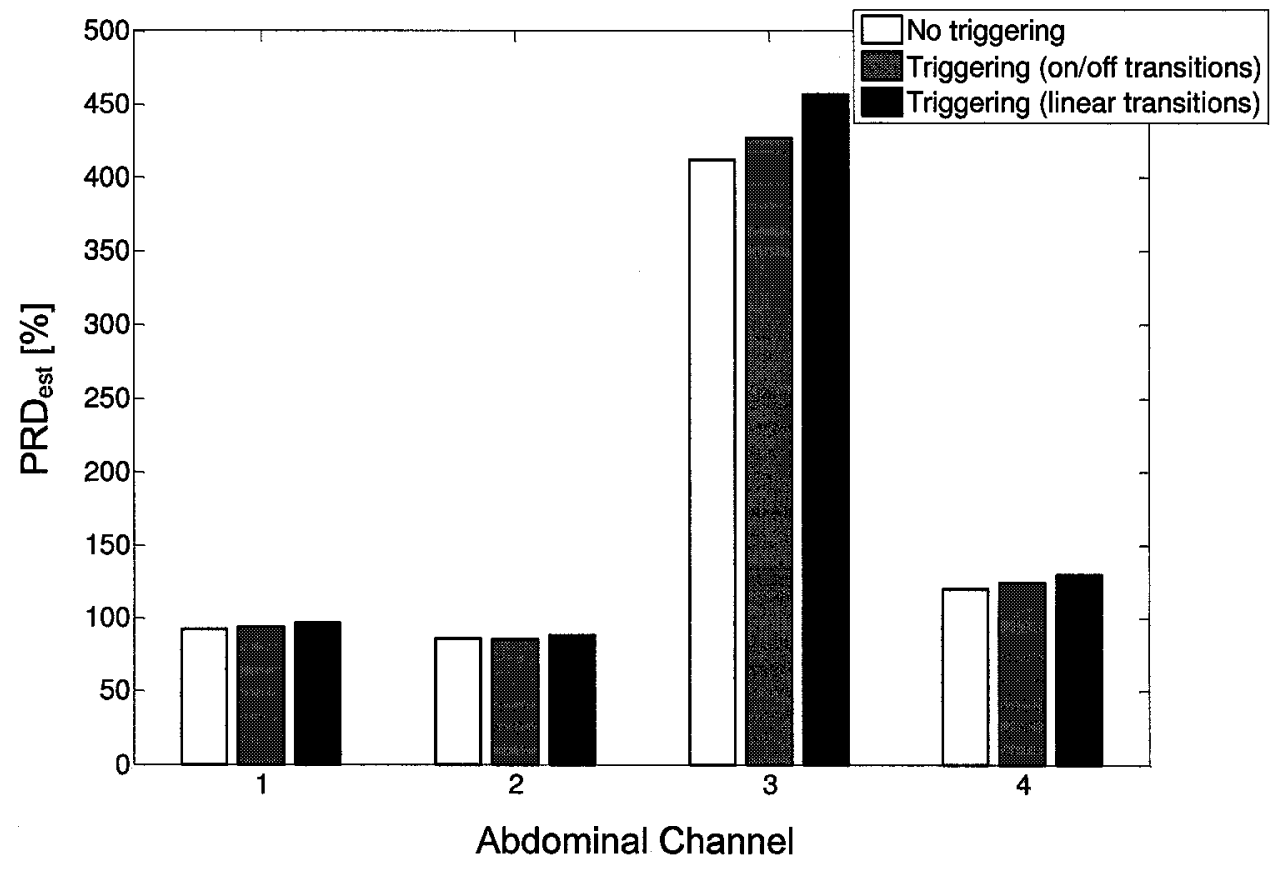

Figure 4-18 PRD $_{\text {est }}$ of abdominal channels after TANC for various triggering modes with an applied SP amplitude of $14 \mathrm{~V}$

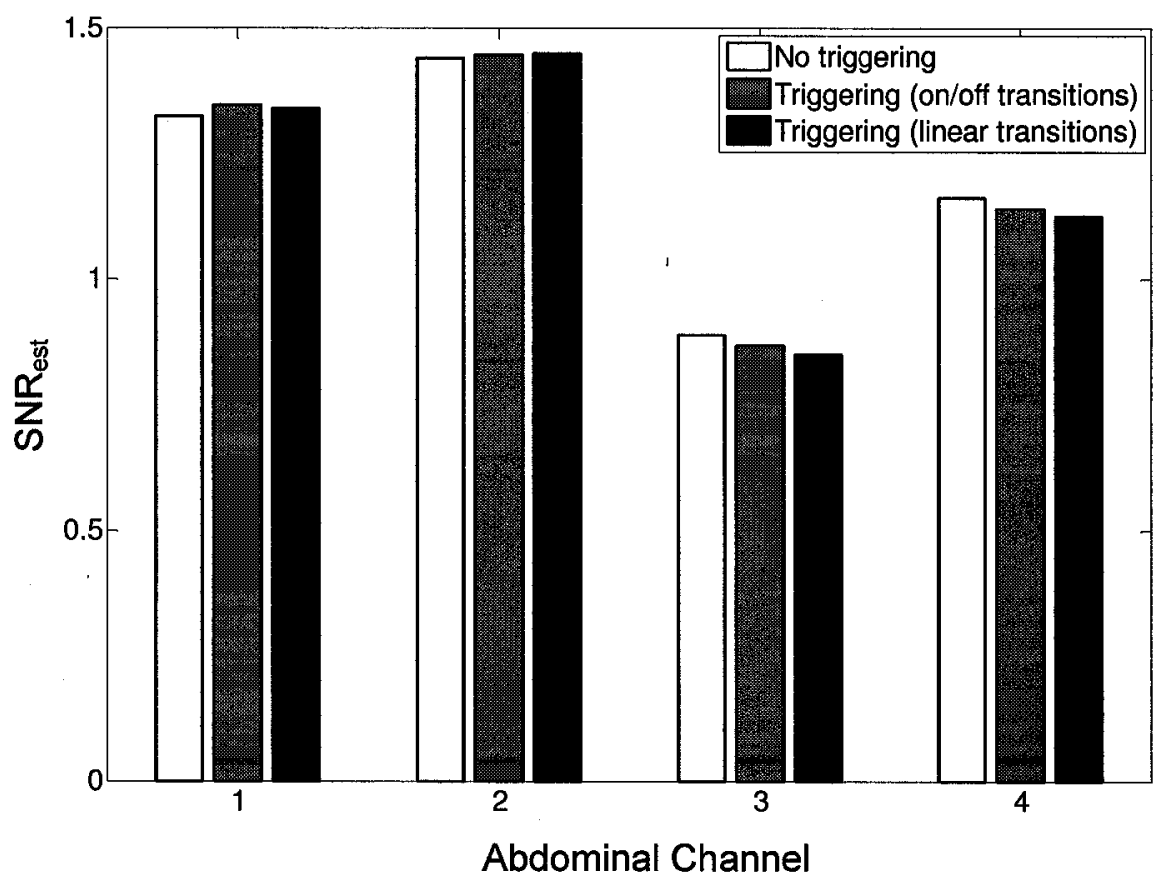

Figure 4-19 SNR $_{\text {est }}$ of abdominal channels after TANC for various triggering modes with an applied SP amplitude of $12 \mathrm{~V}$

86 


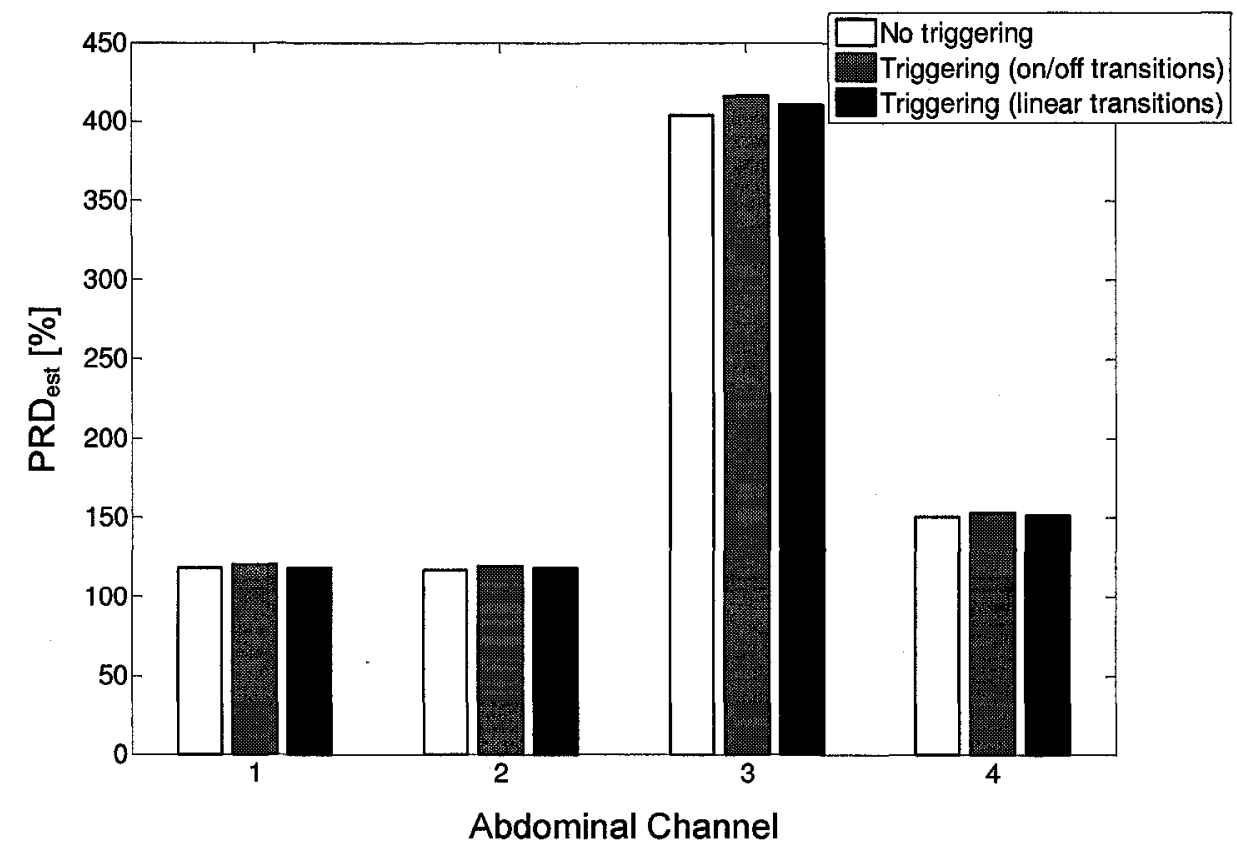

Figure 4-20 PRD $_{\text {est }}$ of abdominal channels after TANC for various triggering modes with an applied SP amplitude of $12 \mathrm{~V}$

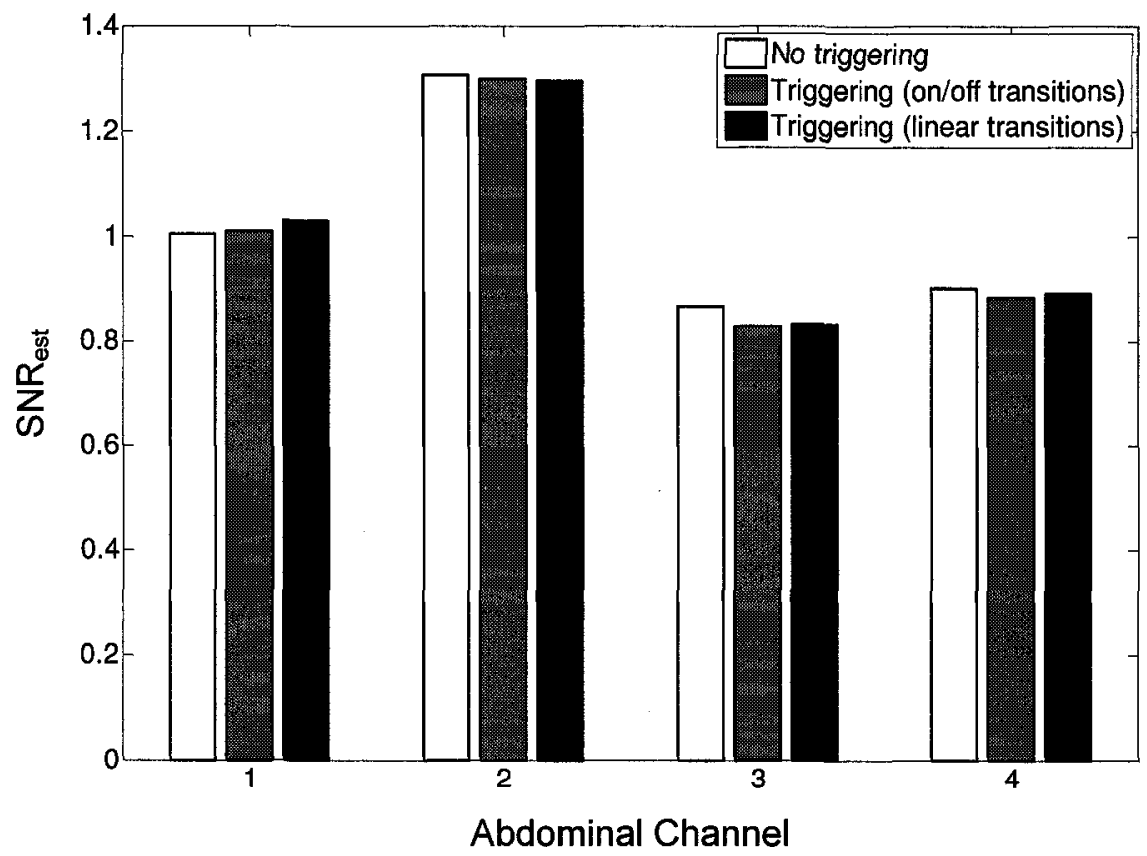

Figure 4-21 SNR $_{\text {est }}$ of abdominal channels after TANC for various triggering modes with an applied SP amplitude of $10 \mathrm{~V}$ 


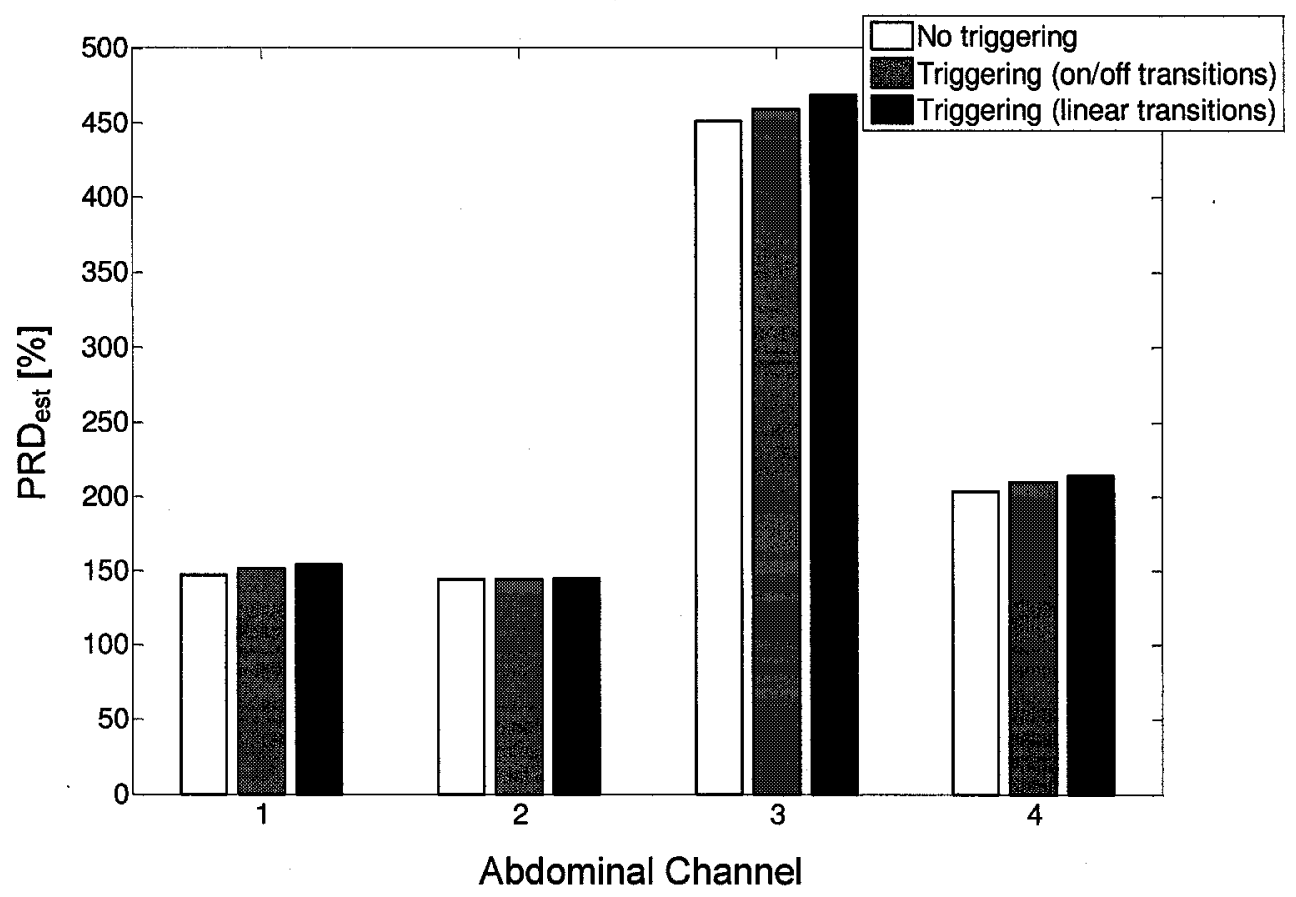

Figure 4-22 PRD $_{\text {est }}$ of abdominal channels after TANC for various triggering modes with an applied SP amplitude of $10 \mathrm{~V}$

Figures 4-23 and 4-24 compare the results of the various triggering modes across the applied SP amplitudes for channel 2, which gave the highest $\mathrm{SNR}_{\text {est }}$, and lowest PRD $_{\text {est, }}$, results. This exposes a key disadvantage of the RLS algorithm; that is, its sensitivity to the input data's SNR. Although many other algorithms exist for FECG extraction, the focus of this work is to establish the usefulness of triggering in the ANC scheme. Extension of this concept to other noise cancellation algorithms is suggested as further research.

It is desirable now to implement a technique to enhance the SNR of the results by exploiting the multiple channels initially acquired. This is performed in the next stage using TASI. 


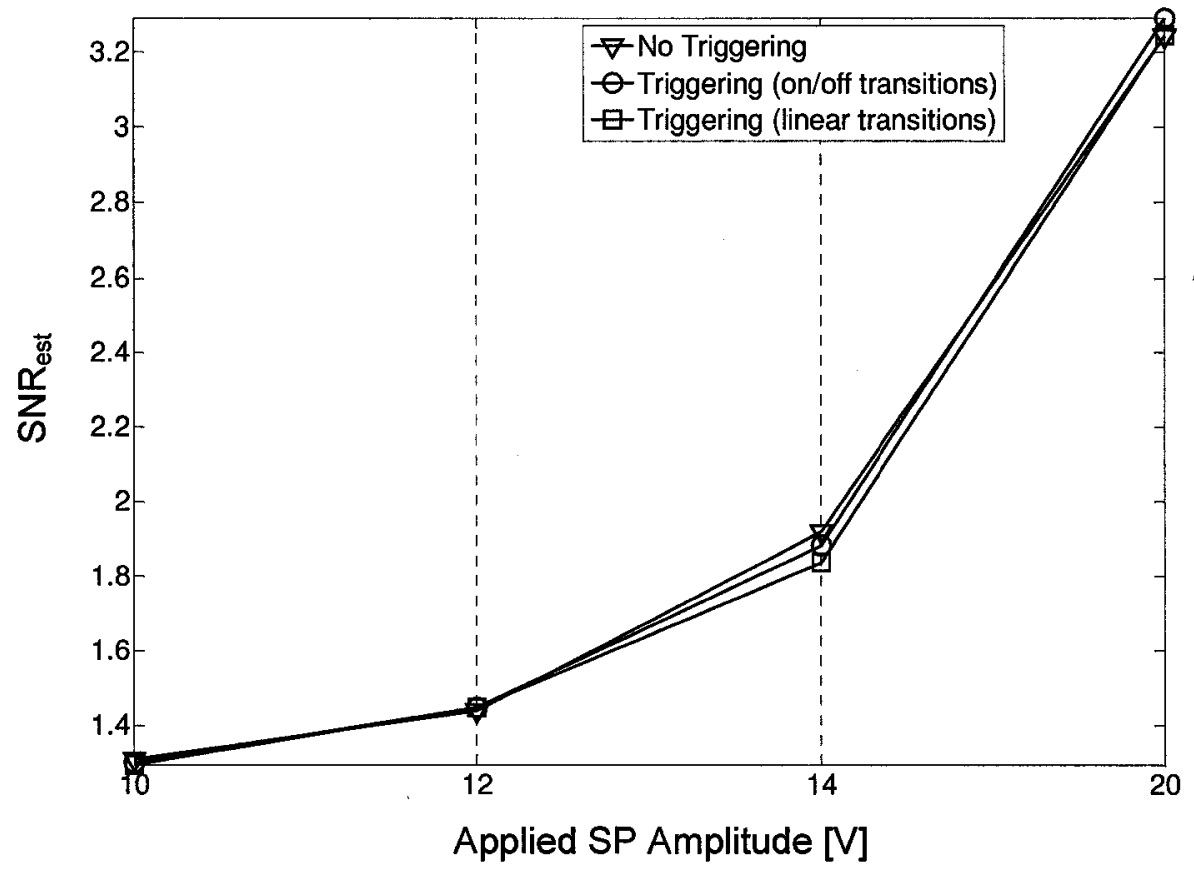

Figure 4-23 SNR $_{\text {est }}$ after TANC for various applied SP amplitudes using channel 2

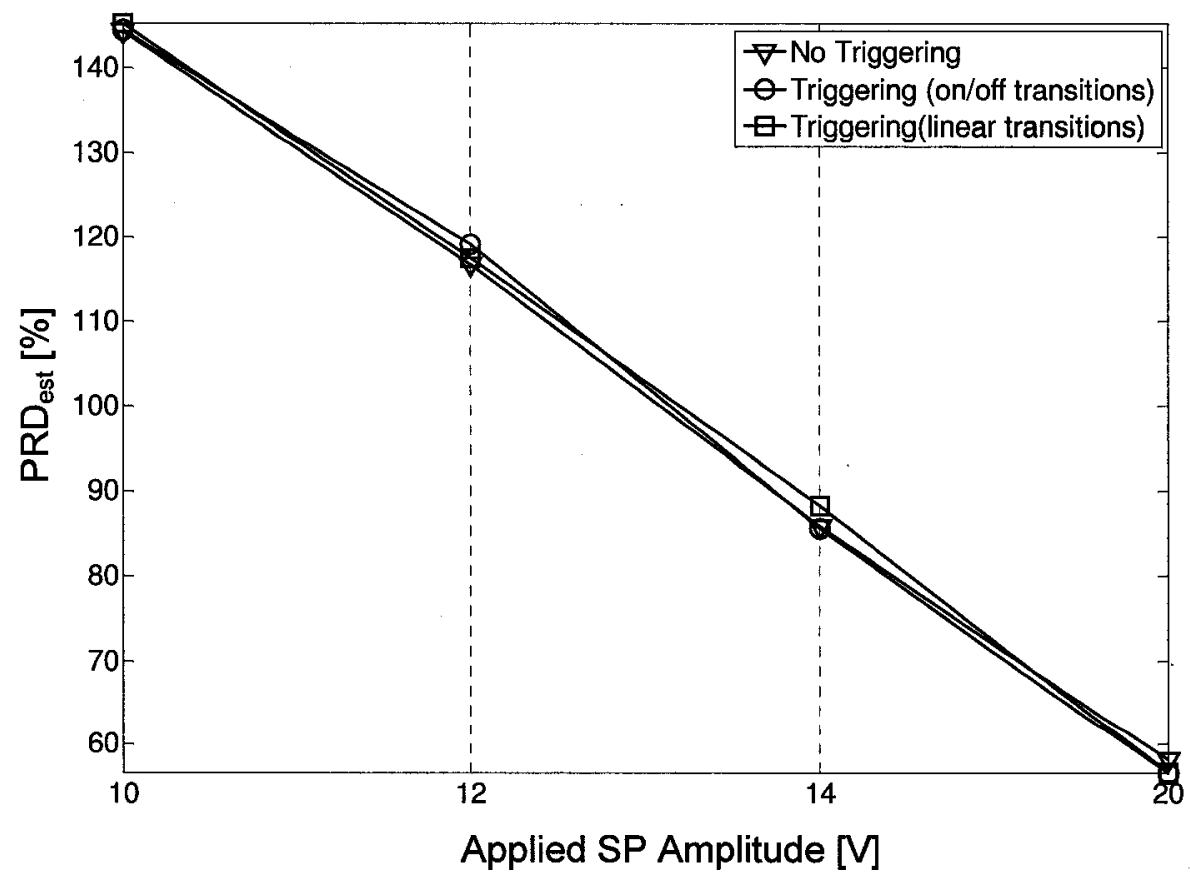

Figure 4-24 PRD $_{\text {est }}$ after TANC for various applied $S P$ amplitudes using channel 2 89 


\subsubsection{Stage 2 Results}

In stage 2, ASI is used to increase the SNR of the SP estimate, assuming uncorrelated noise between the multiple abdominal channels acquired. Similar to stage 1, initial simulations for stage 2 were run using no triggering to investigate an optimal filter order. Again, the RLS adaptive algorithm with adaptive memory (i.e. time-varying forgetting factor ג) was used in our simulations in an FIR filter. An initial $\lambda$ of 0.999 with time-varying values between 0.999 and 0.9999 (increments of 0.0001) were used. Abdominal channel 2 was used as the template model for the primary input to the ASI scheme, since this channel provided the highest $\mathrm{SNR}_{\text {est }}$ after stage 1. The final SP estimate after multichannel ASI is a combination of the estimates given by each of the abdominal channels. As shown in Figures 4-11 and 4-12, the results provided by channel 3 contain more noise power than signal power. This channel is believed to contain very little SP power due to the orthogonal relationship between the acquisition and stimulating electrodes, as explained in Section 4.3; therefore, simulations are performed both with and without channel 3.

Figures 4-25 and 4-26 display the results after stage 2 for varying filter orders using an applied SP amplitude of $20 \mathrm{~V}$. From these figures, it can be seen that the use of channel 3 in the simulations is detrimental to the resulting estimate, giving lower SNR $\mathrm{R}_{\text {est }}$ results and higher PRD est results. At the optimal filter order of 32, the $S N R_{\text {est }}$ of the enhanced signal is 4.14 with channel 3 included, and 7.23 without. This effect implies that care needs to be taken in choosing a satisfactory data acquisition configuration. The circular configuration used in this study may need to be adapted depending on the position of the fetus. All further simulations performed in this study exclude the use of channel 3. 


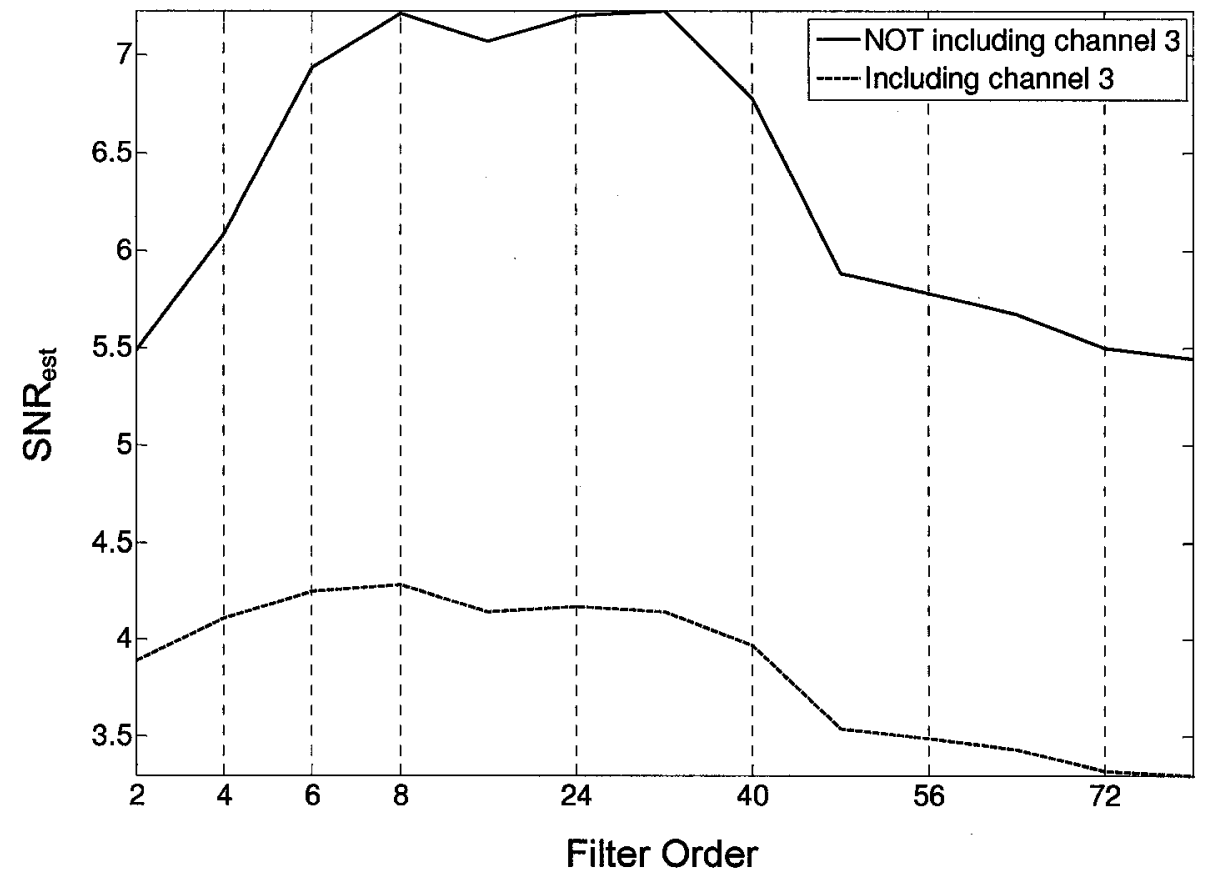

Figure 4-25 SNR $_{\text {est }}$ of SP estimate after ASI for various filter orders with an applied SP amplitude of $20 \mathrm{~V}$ and no triggering

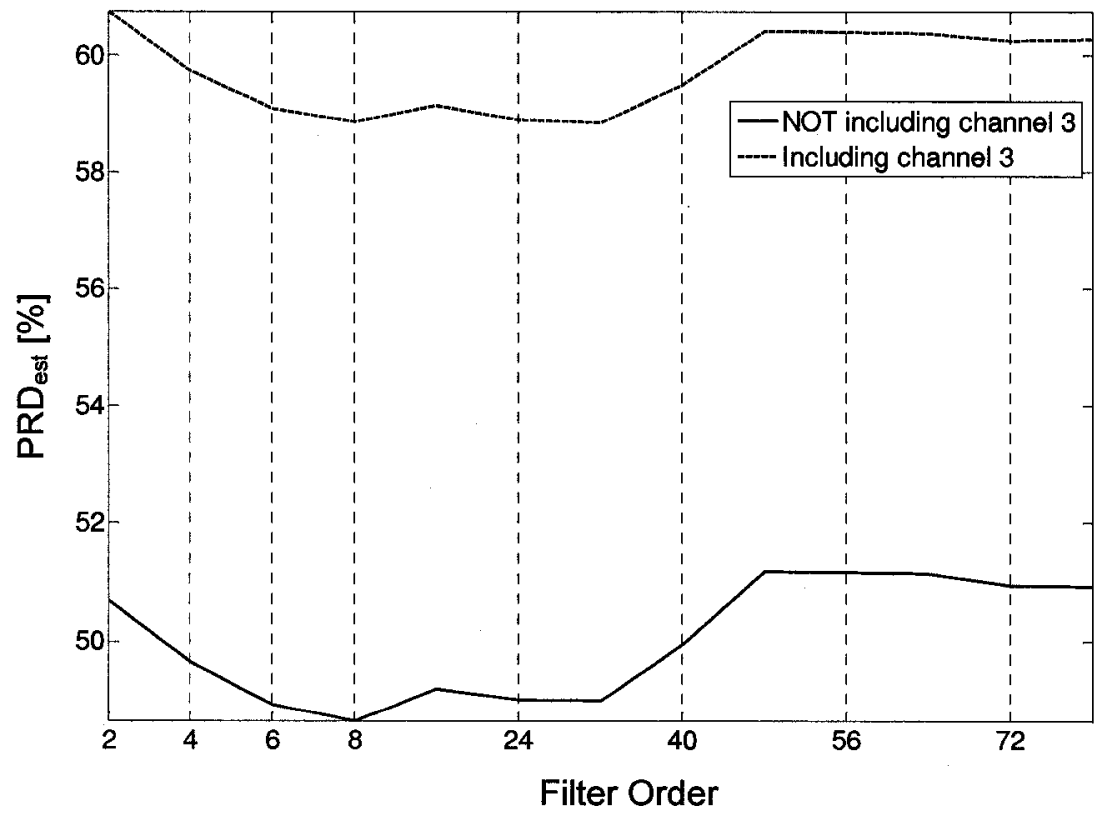

Figure 4-26 PRD est $_{\text {of }}$ SP estimate after ASI for various filter orders with an applied SP amplitude of $20 \mathrm{~V}$ and no triggering

91 
It is encouraging to see that the use of ASI to enhance the SP estimate has indeed improved the $\mathrm{SNR}_{\text {est }}$ from 3.24 to $7.22(\times 2.23$ improvement) using 3 abdominal channels. A similar relationship is exhibited using the $\mathrm{PRD}_{\text {est }}$ performance measure, with an improvement from $58.1 \%$ to $49.0 \%$. We now turn towards the use of triggering in the enhancement scheme. Since it is now the SP which is to be modeled by the adaptive filter (as opposed to the modeling of the subject's ECG in stage 1), triggering is performed by disabling adaptation in between the SPs and enabling adaptation during the SP. In the case of linear transitions, the adapt-enable constraint is enforced during the SP. These are illustrated in Figure 4-27, with the template model output (i.e., channel 2) of stage 1.

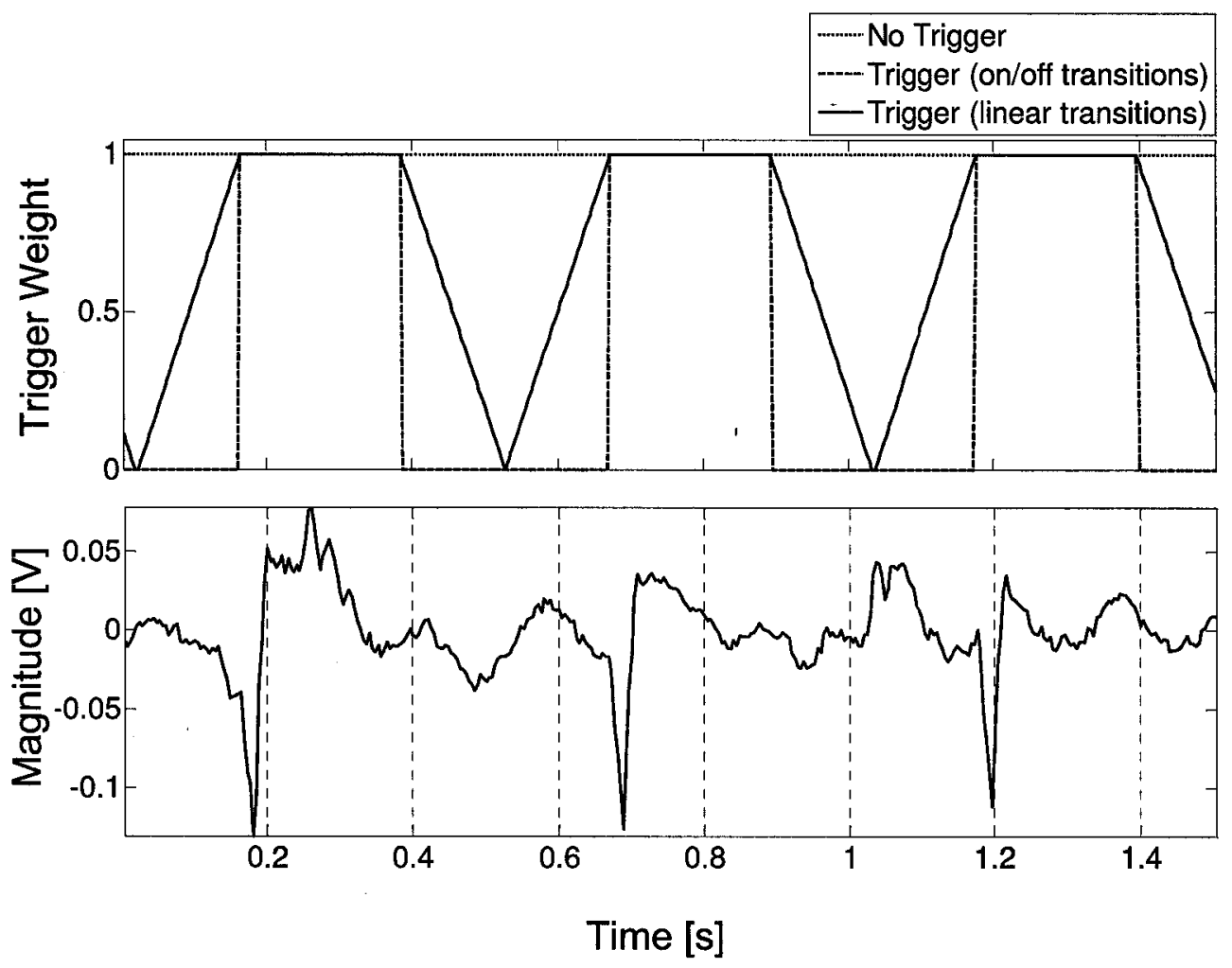

Figure 4-27 Triggering modes used for TASI 
As can be seen in Figures 4-28 and 4-29, the use of triggering in the enhancement scheme has improved the results above that given without triggering. With linear transition weighted triggering, the $\mathrm{SNR}_{\text {est }}$ of the final estimate, shown in Figure 4-28, is 9.83, compared to 7.22 given without triggering. Similarly, in Figure $4-29$, the $P_{R D}$ est has improved from $58.1 \%$ without triggering to $56.6 \%$ with linear transition weighted triggering. This shows that triggering has attenuated uncorrelated noise between the multiple abdominal channels with less attenuation to the desired SP, compared to no triggering.

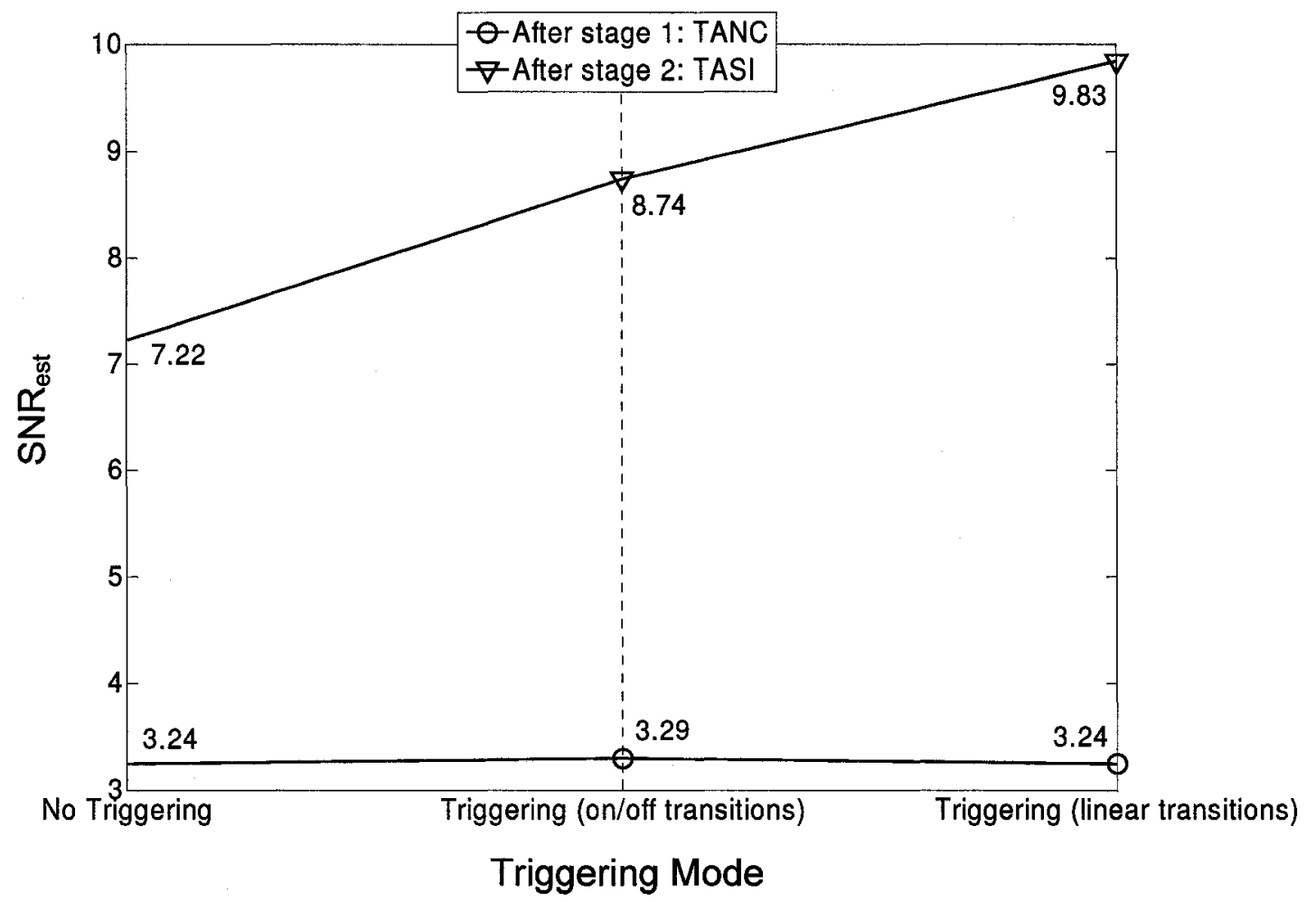

Figure 4-28 SNR $_{\text {est }}$ of final estimate after TASI for various triggering modes with an applied SP amplitude of $20 \mathrm{~V}$ 


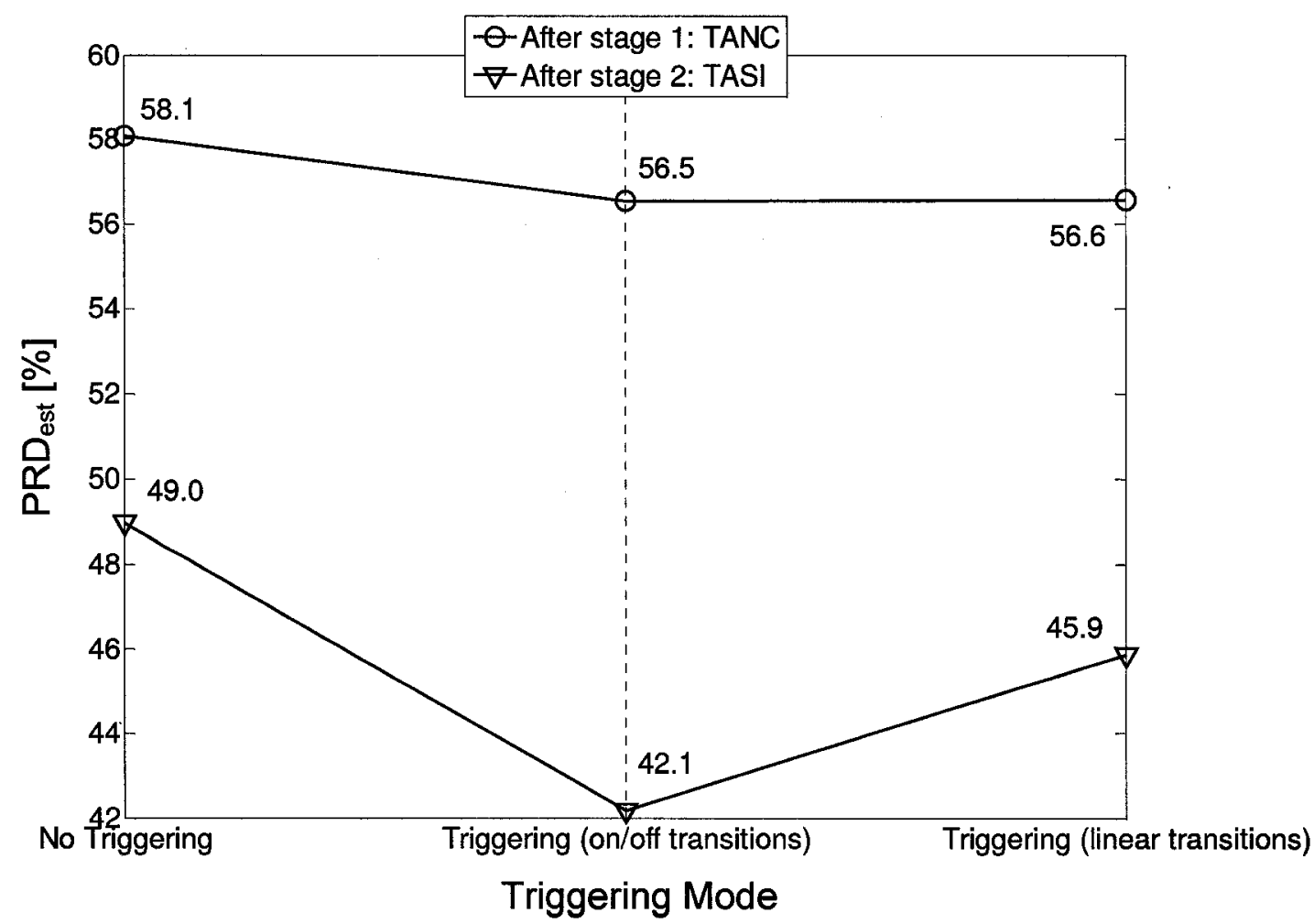

Figure 4-29 $\mathbf{P R D}_{\text {est }}$ of final estimate after TASI for various triggering modes with an applied SP amplitude of $20 \mathrm{~V}$

Figure 4-30 displays a segment of the extracted signal after stage 2 . The results after stage 1, seen in Figure 4-16, are also shown in Figure 4-30 for comparison. Multichannel TASI has severely attenuated the noise power in the extracted signal. The remnants of the subject's ECG, especially apparent at about $0.3 \mathrm{~s}$ and $1.1 \mathrm{~s}$, have also been reduced as a consequence. Although the subject's ECG is correlated between channels, it contains a somewhat 'random' interchannel relationship due to the different tissue-filter effects that the ECG is transformed by as it propagates through the subject to be measured at different locations. Comparing the signal in between the pulses, it can be seen that after TASI, these regions are nearly flat. This is a desired result since the applied SP does not contain any 
activity in these regions. The increase in performance given by triggering using TASI is even more than the results given by TANC in Section 4.5.1.1. The results of stage 1 did show a slight improvement using triggering with on/off transitions $\left(\mathrm{SNR}_{\mathrm{est}}\right.$ increase of $0.04, \mathrm{PRD}_{\text {est }}$ decrease of $1.6 \%$ ), however this is relatively small compared to the improvement given by triggering with linear transitions in stage $2\left(\mathrm{SNR}_{\text {est }}\right.$ increase of $2.61, \mathrm{PRD}_{\text {est }}$ decrease of $3.1 \%$ ). This subjective analysis, together with the encouraging quantitative results that show better results using triggering, justifies the use of triggering. In the next section, this analysis is continued using initial inputs with lower SNRs.
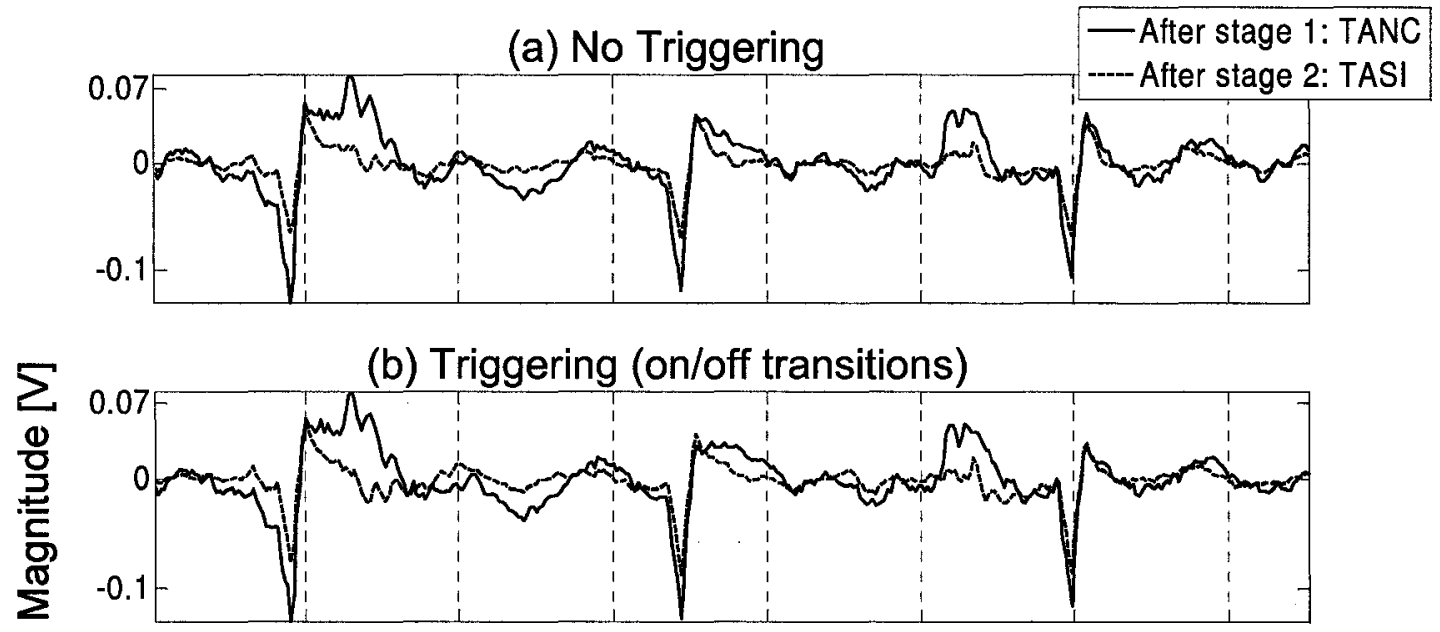

(c) Triggering (linear transitions)

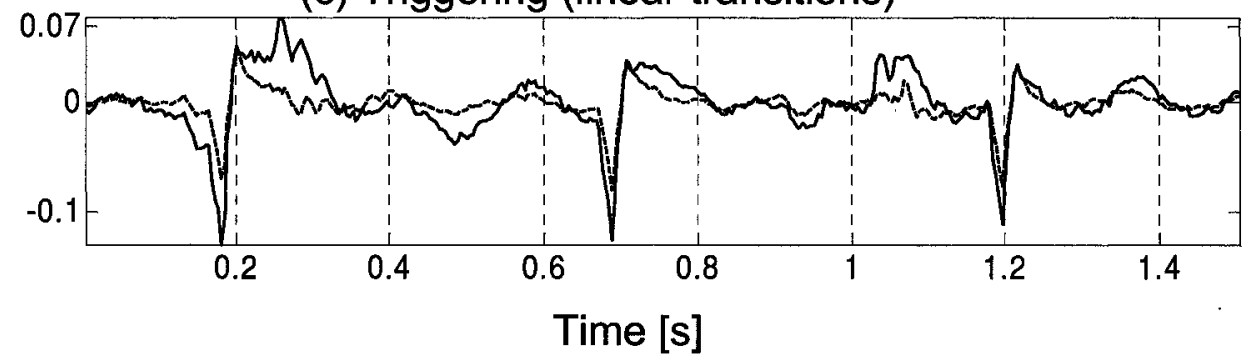

Figure 4-30 Estimated SP after TANC and TASI for various triggering modes with an applied SP amplitude of $20 \mathrm{~V}$ 


\subsubsection{Effect of lowering the SNR of the acquired data}

Further simulations are run using the same filter specifications as that of Section 4.5.2 (i.e., RLS adaptive algorithm, FIR filter order: 32 , initial $\lambda$ : 0.999 with time-varying values between 0.999 and 0.9999 in increments of 0.0001 ). Triggering was used with on/off transitions as well as weighted linear transition triggering with the adapt-enable constraint enforced. These results are compared to ASI with no triggering, as well as to the results of the template model (i. e., channel 2) of stage 1.

Figures 4-31 - 4-36 show the $\mathrm{SNR}_{\text {est }}$ and $\mathrm{PRD}_{\text {est }}$ results of the outputs of TASI using applied SP amplitudes of 14,12 and $10 \mathrm{~V}$. In these figures, triggering with on/off transitions perform better than with linear transitions, unlike the results given using the higher applied SP amplitude of $20 \mathrm{~V}$. The reason for this phenomenon is not understood, and this suggests that a further study into the mathematical implications of the various triggering modes is required. As the SP amplitude is decreased further, as in the case of no triggering in Figure 4-33 and in all cases for Figure 4-35, the use of TASI is actually detrimental to the results. If we look at the SNR est results of stage 1 for these lower SP amplitudes, seen in Figures 4-19 and 4-21, it is evident that except for the higher $\mathrm{SNR}_{\text {est }}$ provided by channel 2 , all other abdominal channels gave $S_{N R}$ values approximately equal or less than 1.0 . Thus, when these results, where the noise power matches or exceeds the signal power, are entered into stage 2 , the adaptive filter is unable to properly model the channel 2 template. This is a similar situation as that given by the low performance in stage 2 when channel 3 was included (Figures 4-25 and 4-26). It seems necessary that a threshold $\mathrm{SNR}_{\text {est }}$ must be met before a signal is input to the TASI scheme. Based on Figure 4-33, this threshold would be 
about 1.45 with the use of triggering, and based on Figure 4-31, about 1.90 without triggering. Therefore, the use of triggering allows inputs with lower $\mathrm{SNR}_{\text {est }}$ to be used to provide an improvement on the final estimate. This analysis is similar using the PRD $\mathrm{est}$ results shown in Figures 4-32, 4-34 and 4-36.

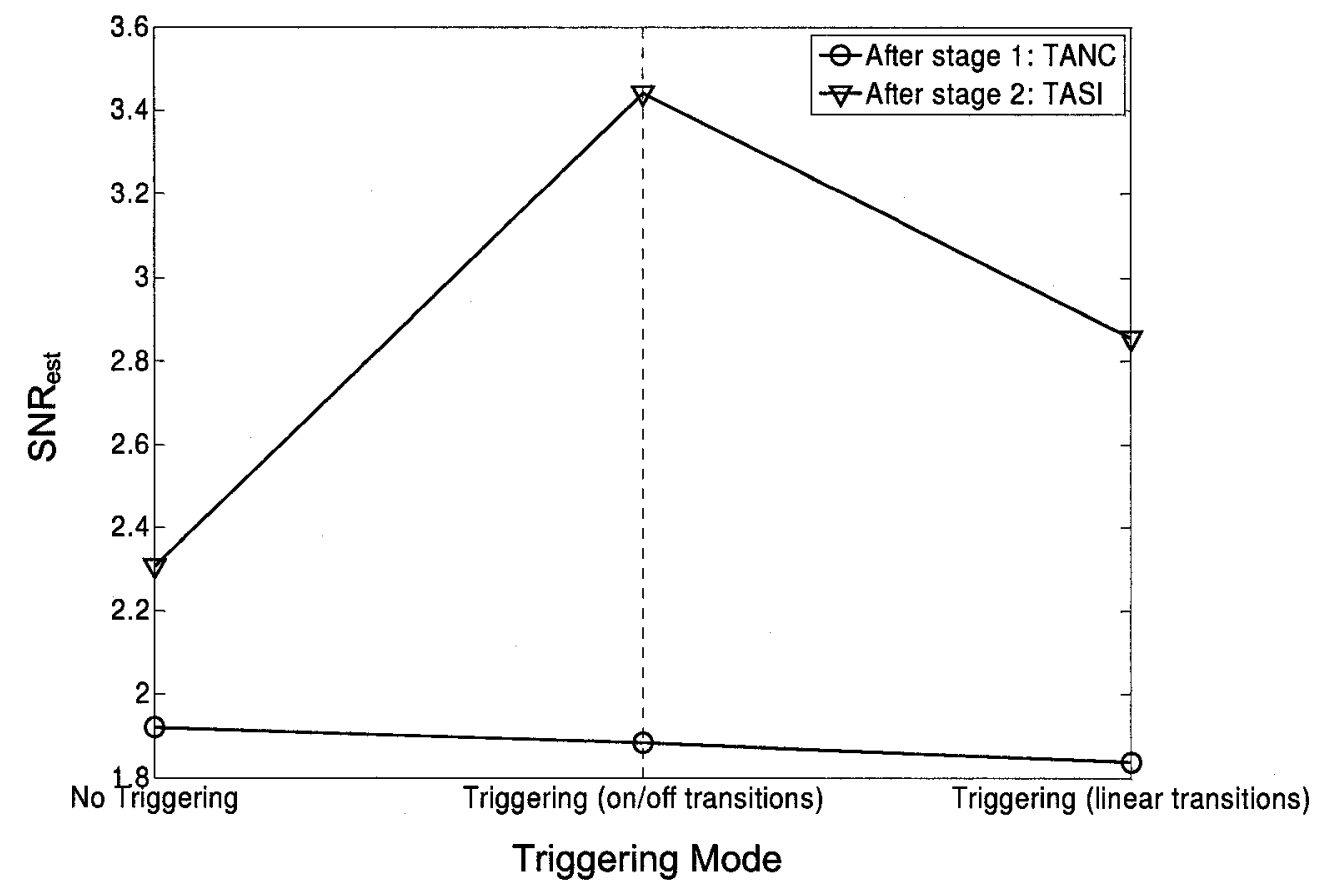

Figure 4-31 SNR $_{\text {est }}$ of final estimate after TASI for various triggering modes and an applied SP amplitude of $14 \mathrm{~V}$ 


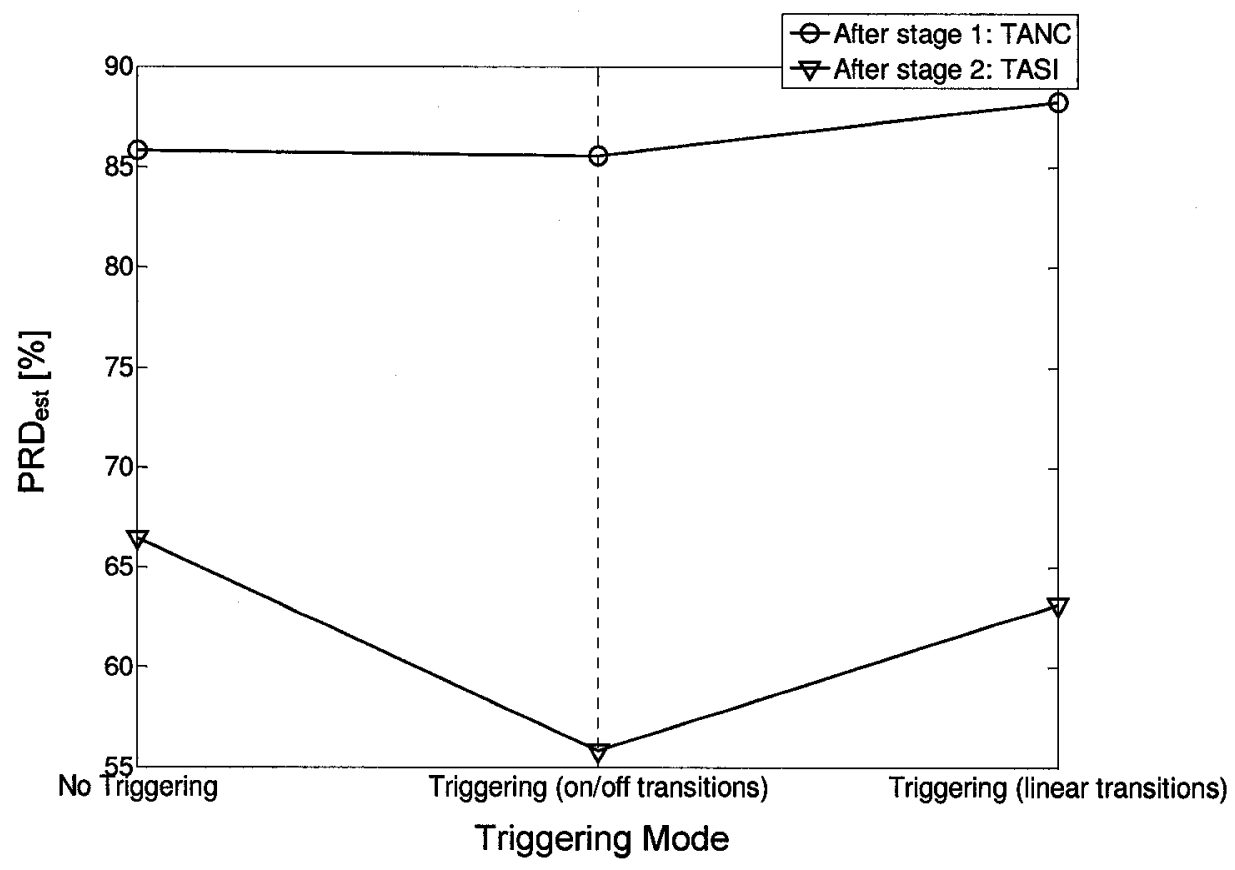

Figure 4-32 $\mathrm{PRD}_{\text {est }}$ of final estimate after TASI for various triggering modes and an applied SP amplitude of $14 \mathrm{~V}$

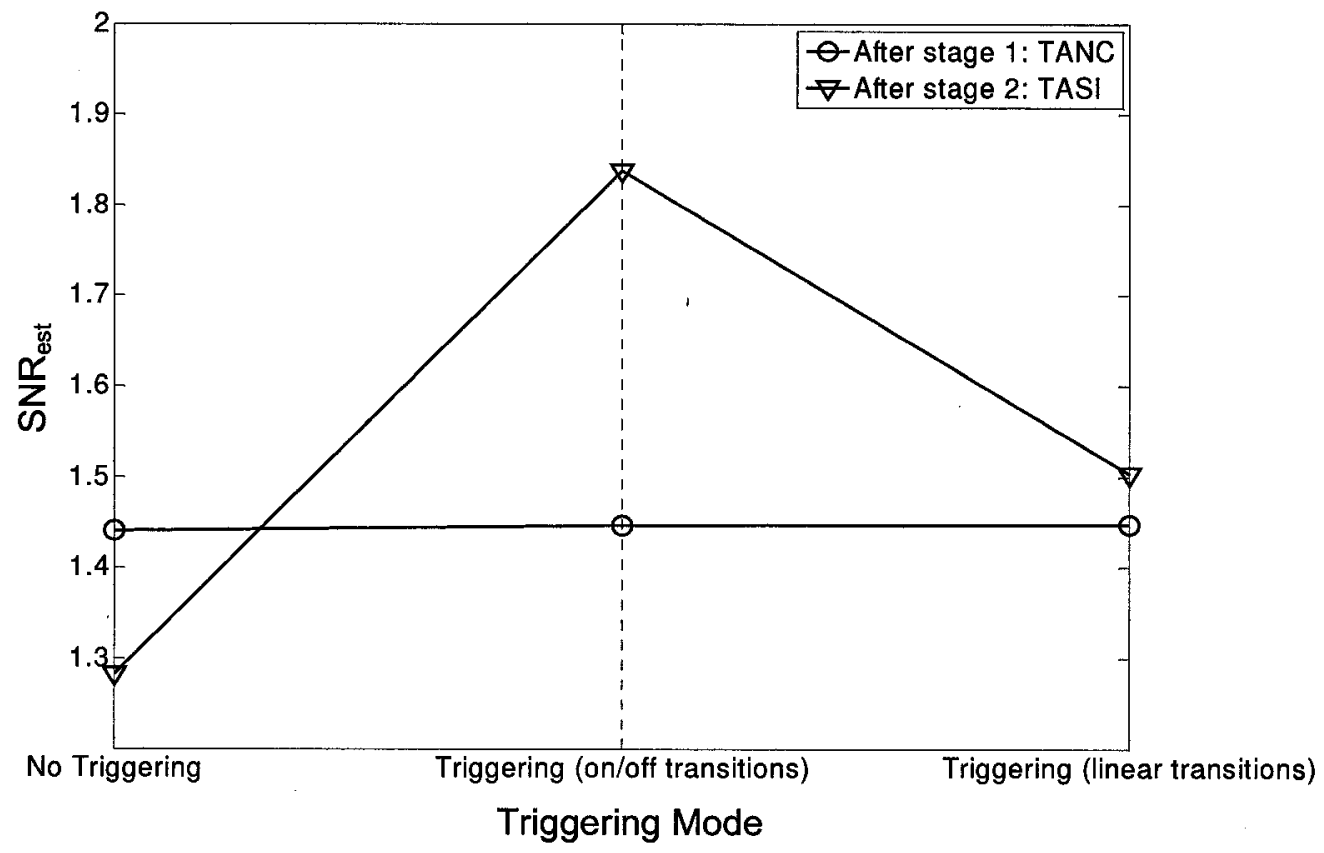

Figure 4-33 SNR $_{\text {est }}$ of final estimate after TASI for various triggering modes and an applied SP amplitude of $12 \mathrm{~V}$

98 


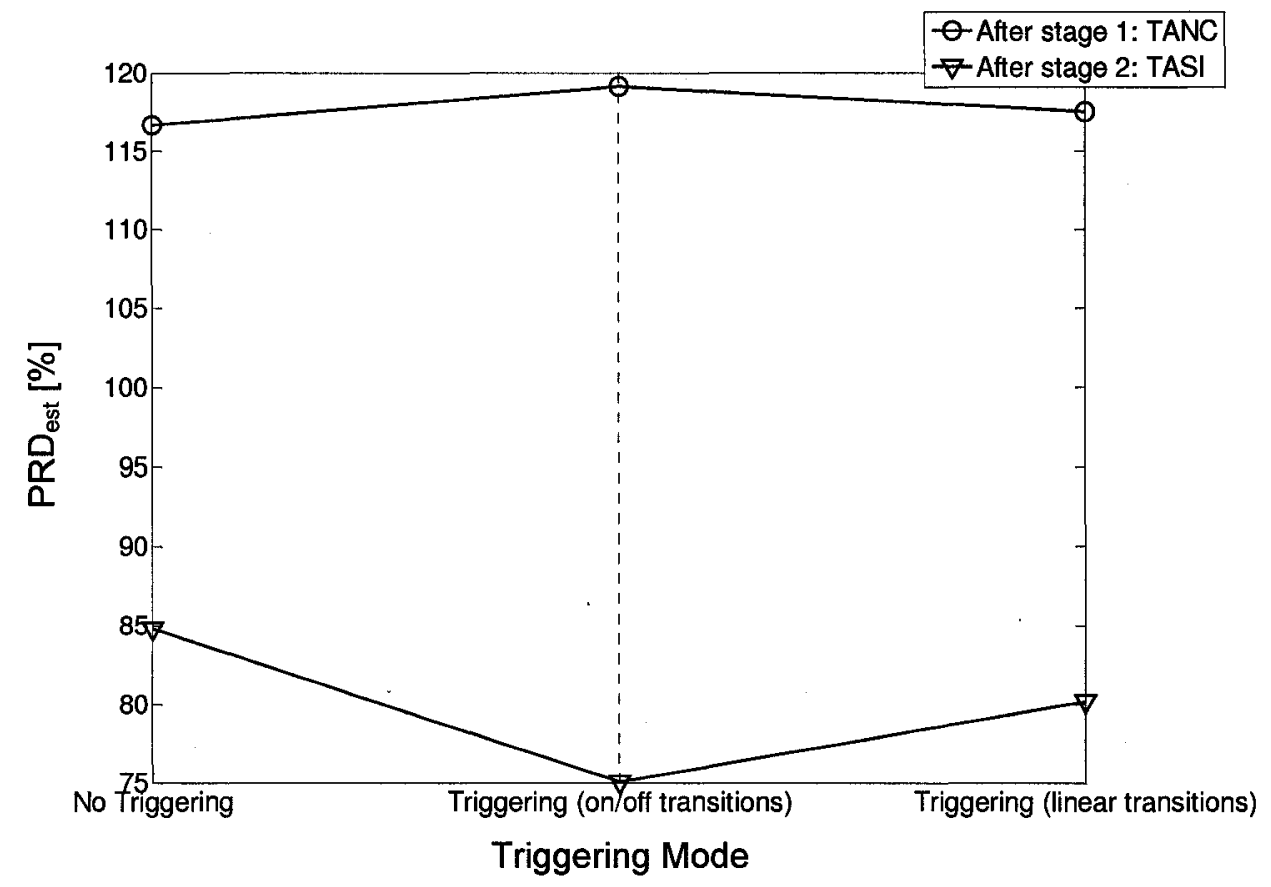

Figure 4-34 PRD $_{\text {est }}$ of final estimate after TASI for various triggering modes and an applied SP amplitude of $12 \mathrm{~V}$

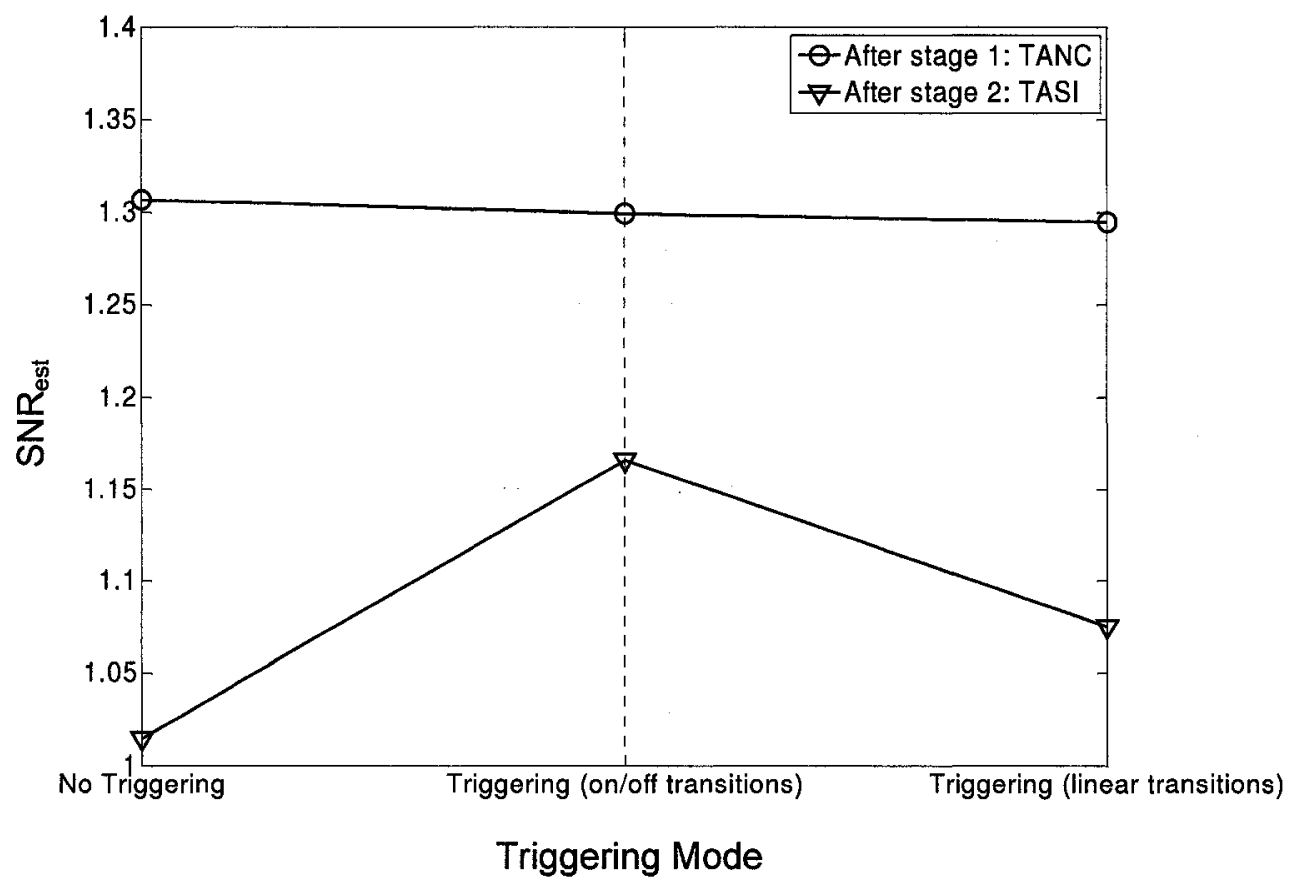

Figure 4-35 SNR $_{\text {est }}$ of final estimate after TASI for various triggering modes and an applied SP amplitude of $10 \mathrm{~V}$

99 


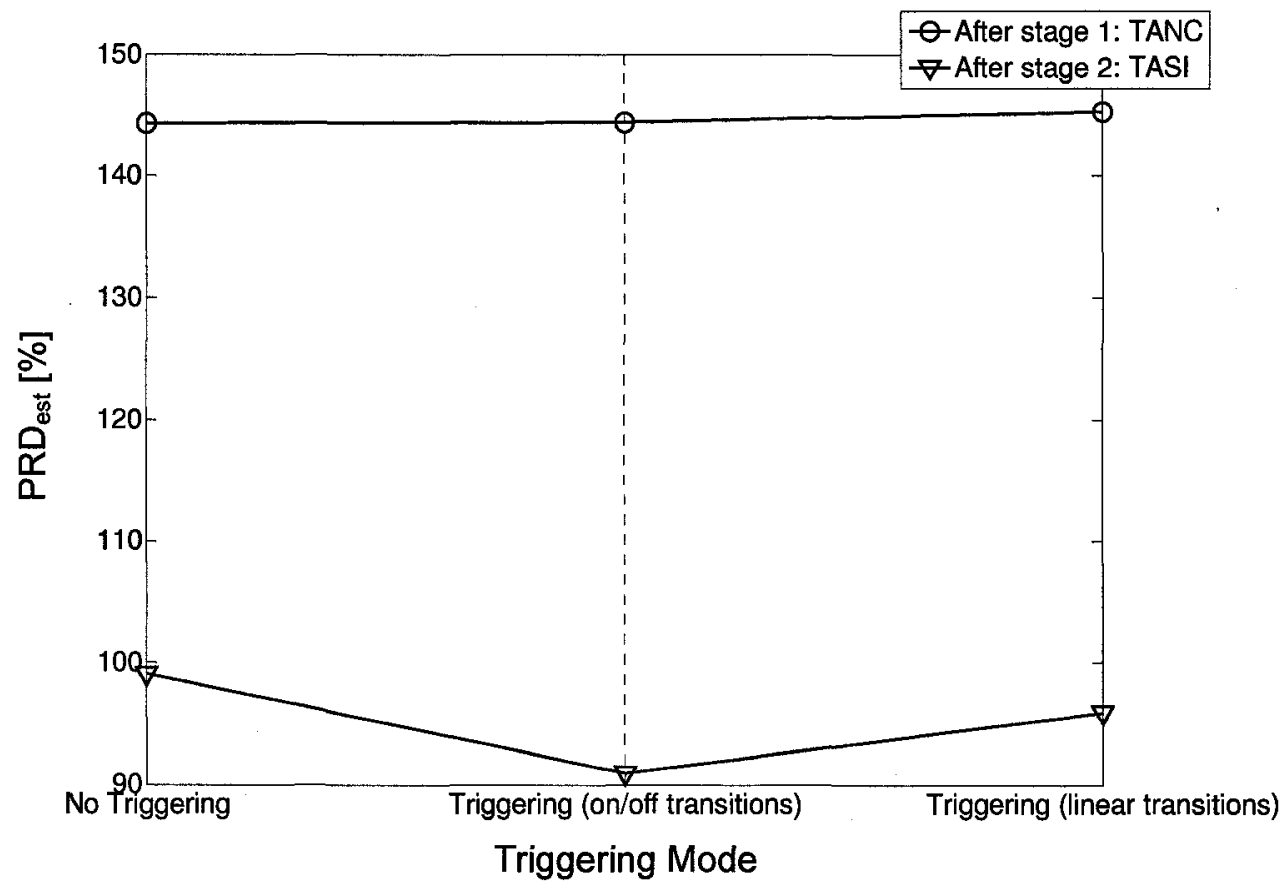

Figure 4-36 PRD $_{\text {est }}$ of final estimate after TASI for various triggering modes and an applied SP amplitude of $10 \mathrm{~V}$

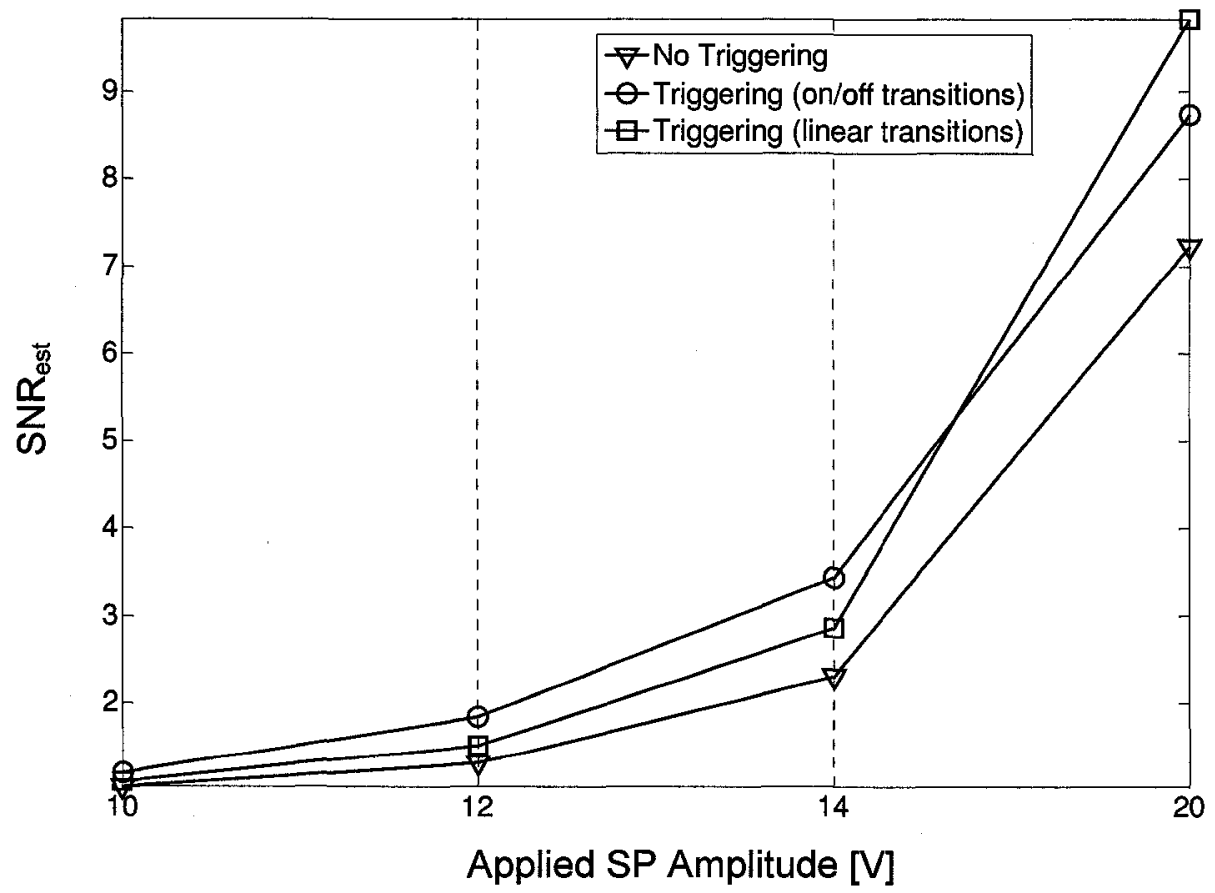

Figure 4-37 SNR $_{\text {est }}$ of final estimate after TASI for various applied SP amplitudes 100 


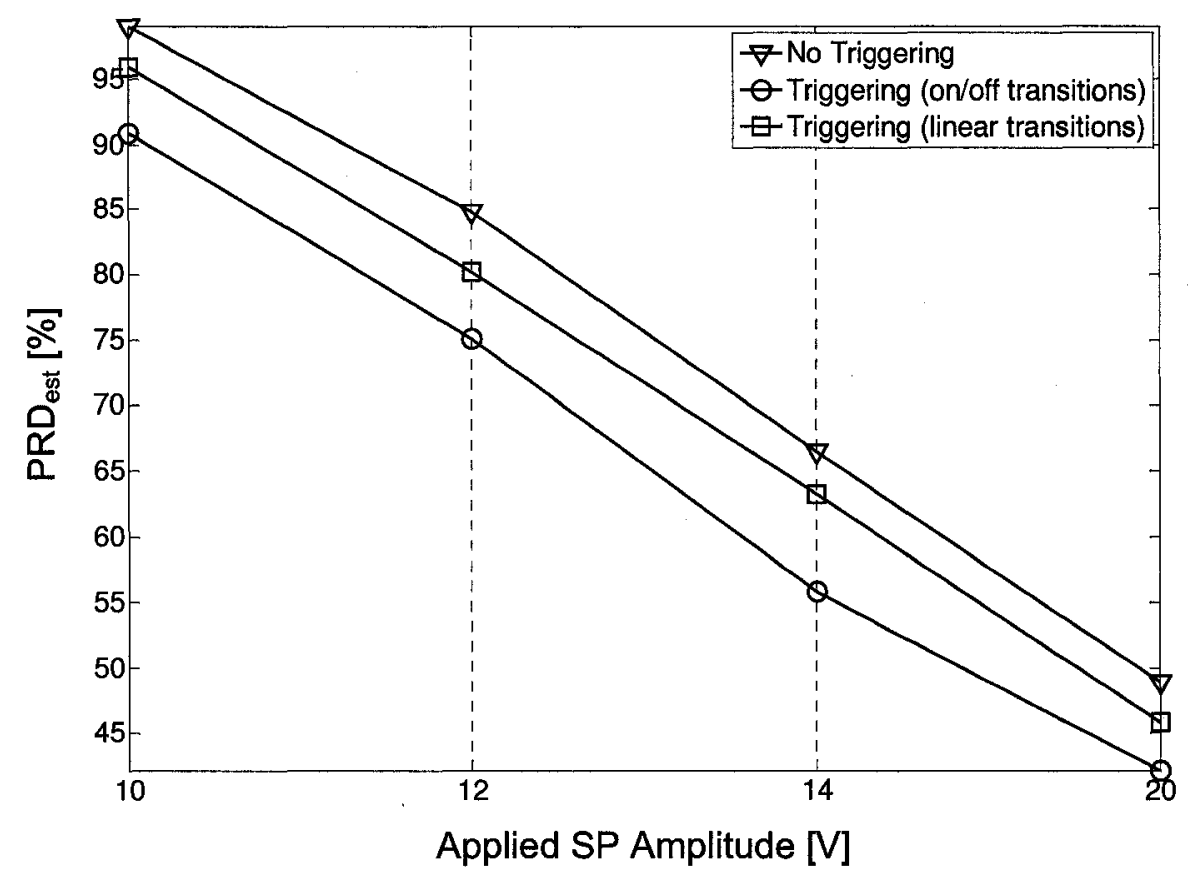

Figure 4-38 PRD $_{\text {est }}$ of final estimate after TASI for various applied SP amplitudes

Figures 4-37 and 4-38 compare the results of the various triggering modes across the applied SP amplitudes for the final estimate. In all cases, the use of triggering outperforms ASI without triggering, both in terms of the $\mathrm{SNR}_{\text {est }}$ and the $\mathrm{PRD}_{\text {est }}$ of the final estimate.

\subsection{Conclusions}

In this chapter, we have proposed an adaptive two-stage multichannel signal extraction and enhancement scheme. We have implemented this system in a linear FIR filter using the RLS adaptation algorithm. An external SP, which was applied to a subject's back, was acquired from the subject's abdomen using a circular array of surface electrodes (4 channels, 8 acquisition sites). The acquired signal is composed of a transformed SP, affected 101 
by propagation through the subject, as well as the subject's ECG. The SP was then extracted from the multiple composite signals using TANC and enhanced using TASI.

In stage 1, TANC was performed using a reference signal acquired from the subject's chest that contains the subject's ECG without the SP. The primary inputs to this scheme are the multiple abdominal channels containing both the subject's ECG and the SP. Simulations were performed using various applied SP amplitudes and triggering modes. SNR $_{\text {est }}$ and $\mathrm{PRD}_{\text {est }}$ were used as performance measures indicating the degree of attenuation of the noise, which includes the subject's ECG and random noise. Results of these simulations show similar $\mathrm{SNR}_{\text {est }}$ and $\mathrm{PRD}_{\text {est }}$ values across the various triggering modes (no triggering, triggering with on/off transitions, and triggering with linear transitions). At an applied SP amplitude of $20 \mathrm{~V}$, triggering with on/off transitions gave an improvement in SNR $\mathrm{Sst}_{\text {ef }}$ o.04 $\left(\mathrm{SNR}_{\mathrm{est}}=3.24\right)$ and a $\mathrm{PRD}_{\mathrm{est}}$ improvement of $1.6 \%\left(\mathrm{PRD}_{\mathrm{est}}=56.5 \%\right)$ over $\mathrm{ANC}$ without triggering, while triggering with linear transitions gave the same $\mathrm{SNR}_{\mathrm{est}}$ as no triggering $\left(\mathrm{SNR}_{\mathrm{est}}=3.20\right)$ and a $\mathrm{PRD}_{\text {est }}$ improvement of $1.6 \%\left(\mathrm{PRD}_{\mathrm{est}}=56.5 \%\right)$. This indicates an equal or better performance with the use of triggering in this study. At lower applied SP amplitudes, the results decrease expectedly, again with similar results between triggering modes.

In stage 2, we attempt to further increase the SNR of the estimate by exploiting the multiple channels using a TASI scheme. The reference input to this scheme was a manually chosen estimated signal output from stage 1 to be used as a template. The primary inputs consisted of all other signal estimate outputs (i.e., except for the template signal). Simulations were again performed using the various applied SP amplitudes and triggering 
modes. The results indicate better performance using triggering across the various applied SP amplitudes. An $\mathrm{SNR}_{\text {est }}$ improvement of 2.61 was provided by triggering with linear transitions $\left(\mathrm{SNR}_{\mathrm{est}}=9.83\right)$ over no triggering $\left(\mathrm{SNR}_{\mathrm{est}}=7.22\right)$ at an applied SP amplitude of 20 V. Similarly, a PRD $_{\text {est }}$ improvement of $3.1 \%$ was achieved. Subjective analysis confirms that the use of multichannel ASI severely attenuates the noise levels of the estimate, providing a better SP shape.

It is realized, however, that in order for an $\mathrm{SNR}_{\mathrm{est}}$ improvement to be achieved, a threshold SNR $_{\text {est }}$ level must be met for the inputs to the TASI scheme. This threshold $\mathrm{SNR}_{\text {est }}$ was shown to be lower when triggering is used, indicating a more robust system that can handle inputs with lower SNRs compared to ASI without triggering.

Given the promising results of this multichannel adaptive scheme with triggering, extending the study to perform analysis using real data from pregnant subjects is a desirable next phase. 


\section{Chapter 5 \\ Simulation Study with Real Data}

\subsection{Introduction}

In Chapter 4, we examined the use of a multichannel system that used TANC to extract an SP from an abdominal composite signal, and then TASI to enhance the SP estimate. It was shown that the use of triggering provides a greater degree of attenuation of the unwanted interferences, which include the subject's ECG and random noise sources. However, there are some differences between using real data versus the use of an SP to represent the FECG. Firstly, the SP is applied to the subject at constant intervals, while it is well understood that the FECG contains variability in its rate. Secondly, although the frequency information of the acquired SP data is within the desired range, it does not exactly correspond to the frequency information contained in an FECG. In this chapter, we examine the multichannel extraction and enhancement scheme described in Section 4.2, using real MECG and FECG data from a pregnant subject as inputs.

\subsection{Experimental Methods}

Real data were contributed to a public data set by Dr. Lieven De Lathauwer and obtained for use in this study from [35]. This data were collected noninvasively from a pregnant subject. In total, 8 channels of data were acquired, consisting of 5 abdominal and 3 thoracic channels, for a duration of $10 \mathrm{~s}$ with $f_{s}=250 \mathrm{~Hz}$. Figure 5-1 displays a segment of 
the data from all abdominal channels and a single thoracic channel. Unfortunately, specific electrode placement configuration for this data set could not be obtained; however, the authors involved in the data collection explain in [36] that measurements were performed using $\mathrm{Ag} / \mathrm{AgCl}$ surface electrodes, and the data acquired were filtered using a first-order high-pass filter with $\tau_{0}=10$ or $50 \mathrm{~ms}$, and a second-order low-pass filter with $f_{0}=70 \mathrm{~Hz}$.

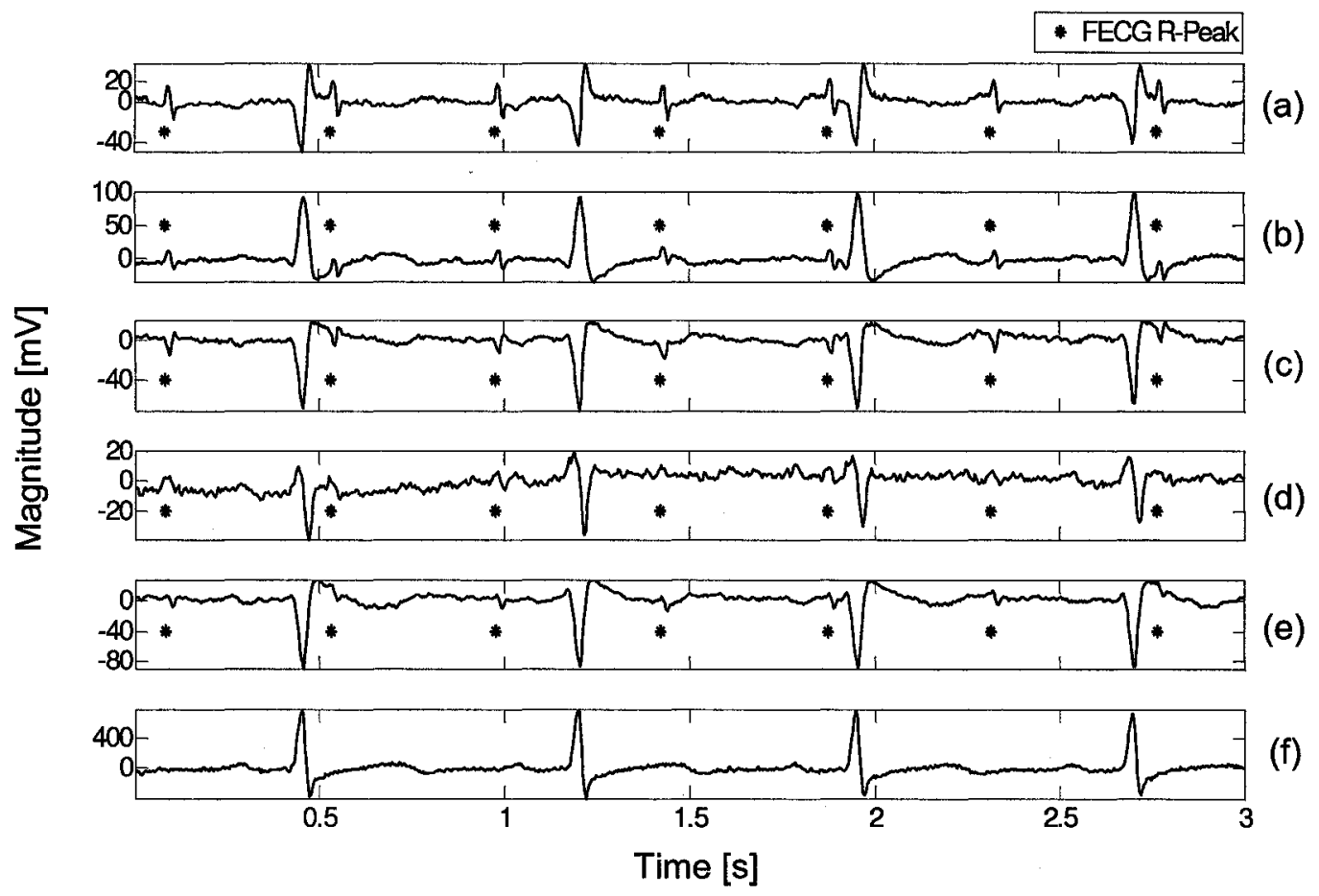

Figure 5-1 Real ECG data: (a)-(e) Abdominal channels 1-5, respectively, containing MECG and FECG data, (f) Thoracic channel containing only MECG data

\subsection{Performance Measure}

Similar to the study presented in Chapter 4 , there is no ideal pulse with which to compare the system's estimated output. Thus, the secondary performance measure, $\mathrm{SNR}_{\mathrm{est}}$, described in Section 4.4.1, will again be used to evaluate the results obtained in this chapter. 
However, due to the limited data length of $10 \mathrm{~s}$ (with 23 FECG pulses), a reasonable timeaveraged FECG cannot be obtained from the acquired data. For this reason, the duration of the FECG, which is required in the calculation of Equation 4.3, was determined visually from the acquired data. The FECG was found to be $0.28 \mathrm{~s}$ in duration, with $0.1 \mathrm{~s}$ occurring before the FECG R-peak and $0.18 \mathrm{~s}$ afterwards. The $\mathrm{SNR}_{\text {est }}$ performance measure is calculated based on data occurring after $2 \mathrm{~s}$ to ensure that the adaptive filter has converged (which was verified visually). This translates to $8 \mathrm{~s}$ of data for stage 1 results (2000 samples using $f_{s}=250 \mathrm{~Hz}$, with $18 \mathrm{FECG}$ pulses), and $6 \mathrm{~s}$ for stage 2 results (1500 samples with $13 \mathrm{FECG}$ pulses).

The secondary $\mathrm{PRD}_{\text {est }}$ performance measure, described in Section 4.4.2, cannot be applied in this chapter because it requires a reasonable time-averaged FECG to be used as an ideal signal in Equation 3.16.

\subsection{Results}

In this section, we examine the results of the two-stage extraction and enhancement scheme, detailed in Section 4.2, with real MECG and FECG signal inputs.

\subsubsection{Stage 1 Results}

Simulations for stage 1 (i.e., TANC) were initially run using no triggering to investigate an optimal filter order. The RLS adaptive algorithm with adaptive memory was used in our simulations with an FIR filter. An initial $\lambda$ of 0.999 with time-varying values 
between 0.999 and 0.9999 (with increments of 0.0001 ) was used. Figure 5-2 displays the calculated SNR $_{\text {est }}$ values of the FECG estimate for varying filter orders.

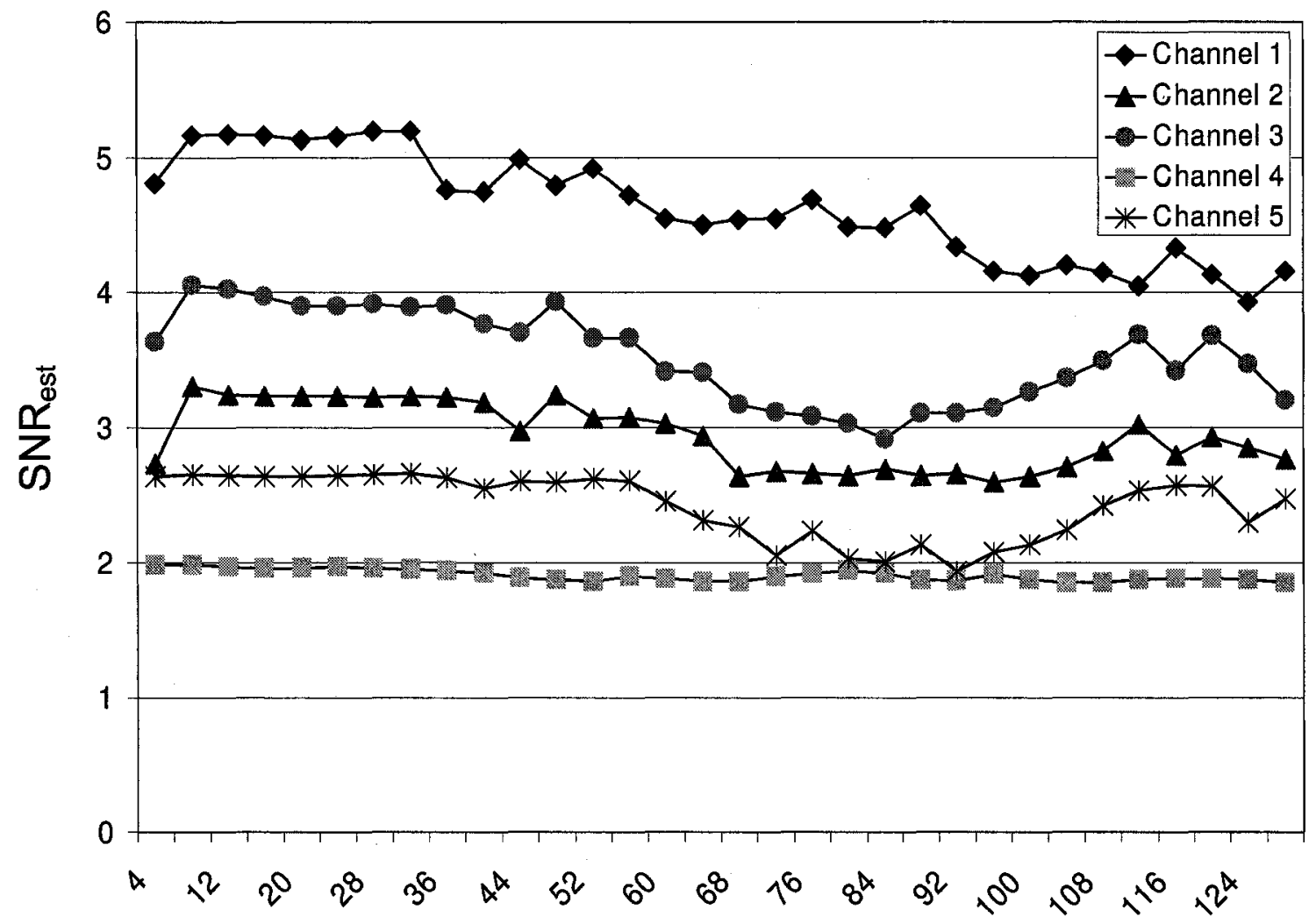

Filter Order

Figure 5-2 SNR $_{\text {est }}$ of abdominal channels after ANC for various filter orders and no triggering

Further simulations were run using TANC, now incorporating triggering, and using the RLS adaptive algorithm with adaptive memory in an FIR filter. The filter used had an initial $\lambda$ of 0.999 with time-varying values between 0.999 and 0.9999 (increments of 0.0001 ). The filter order was initially chosen as 28 , shown to be empirically optimal in Figure 5-2 using no triggering. However, it was found that the limited input data length available restricted the adaptive filter from fully converging. Thus, the FECG is not extracted. A 107 
solution to this is to run the data through the adaptive filter multiple times, and this did indeed lead to convergence of the adaptive filter and extraction of the FECG. Unfortunately, this technique also introduces more gradient noise to the estimated FECG, reducing its $\mathrm{SNR}_{\text {est }}$ Simulations were thus run using various filter orders with triggered adaptation to identify an optimal order capable of extracting the FECG with no added distortion due to multiple passes through the filter. Triggering was used with on/off transitions, as well as weighted linear transition triggering with the adapt-disable constraint enforced and a minimum trigger weight of 0.05 . The results for filter orders capable of extracting the FECG in a single pass are shown in Figures 5-3 and 5-4, respectively.

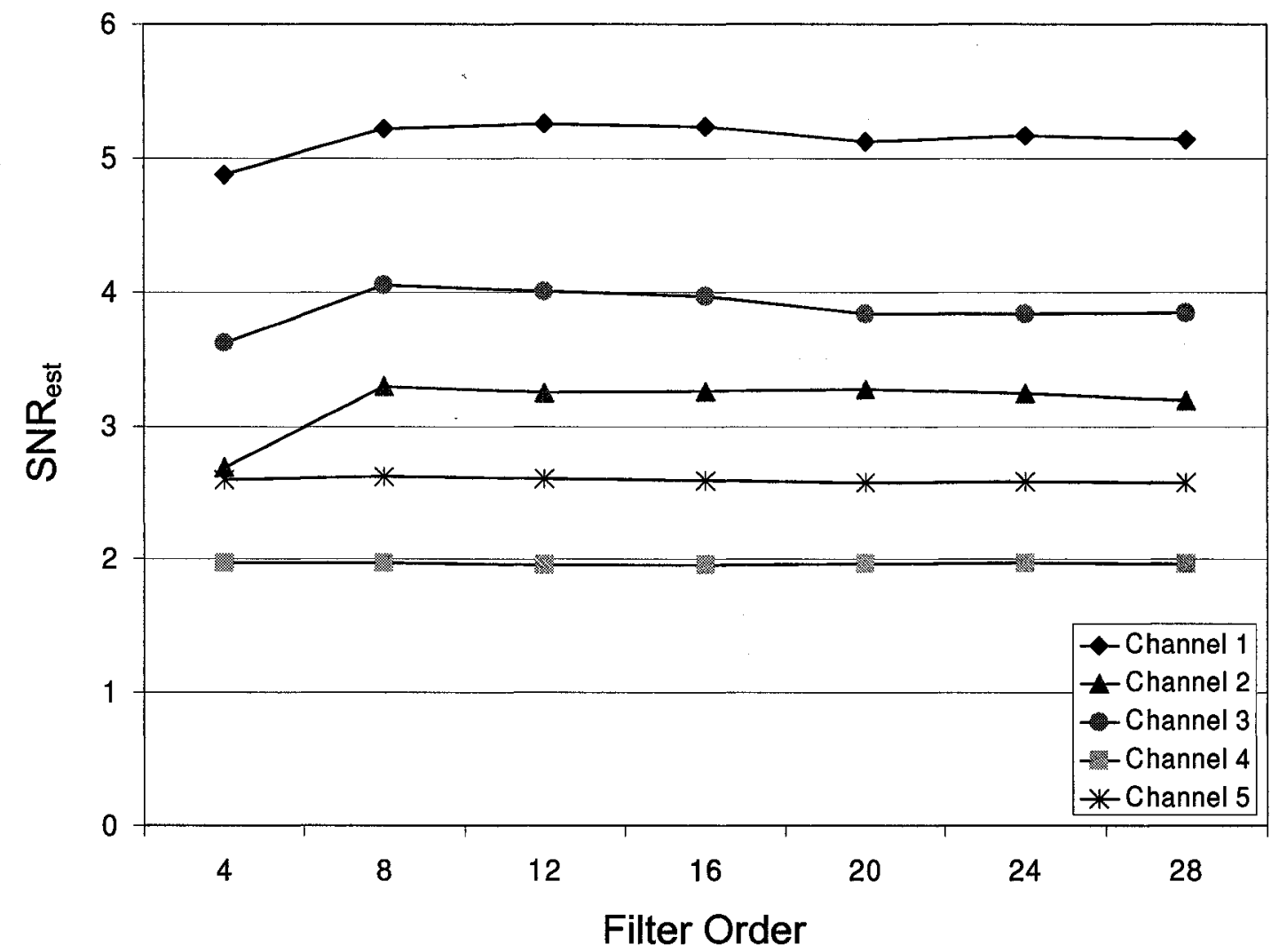

Figure 5-3 SNR $_{\text {est }}$ of abdominal channels after TANC for various filter orders using triggering with on/off transitions 


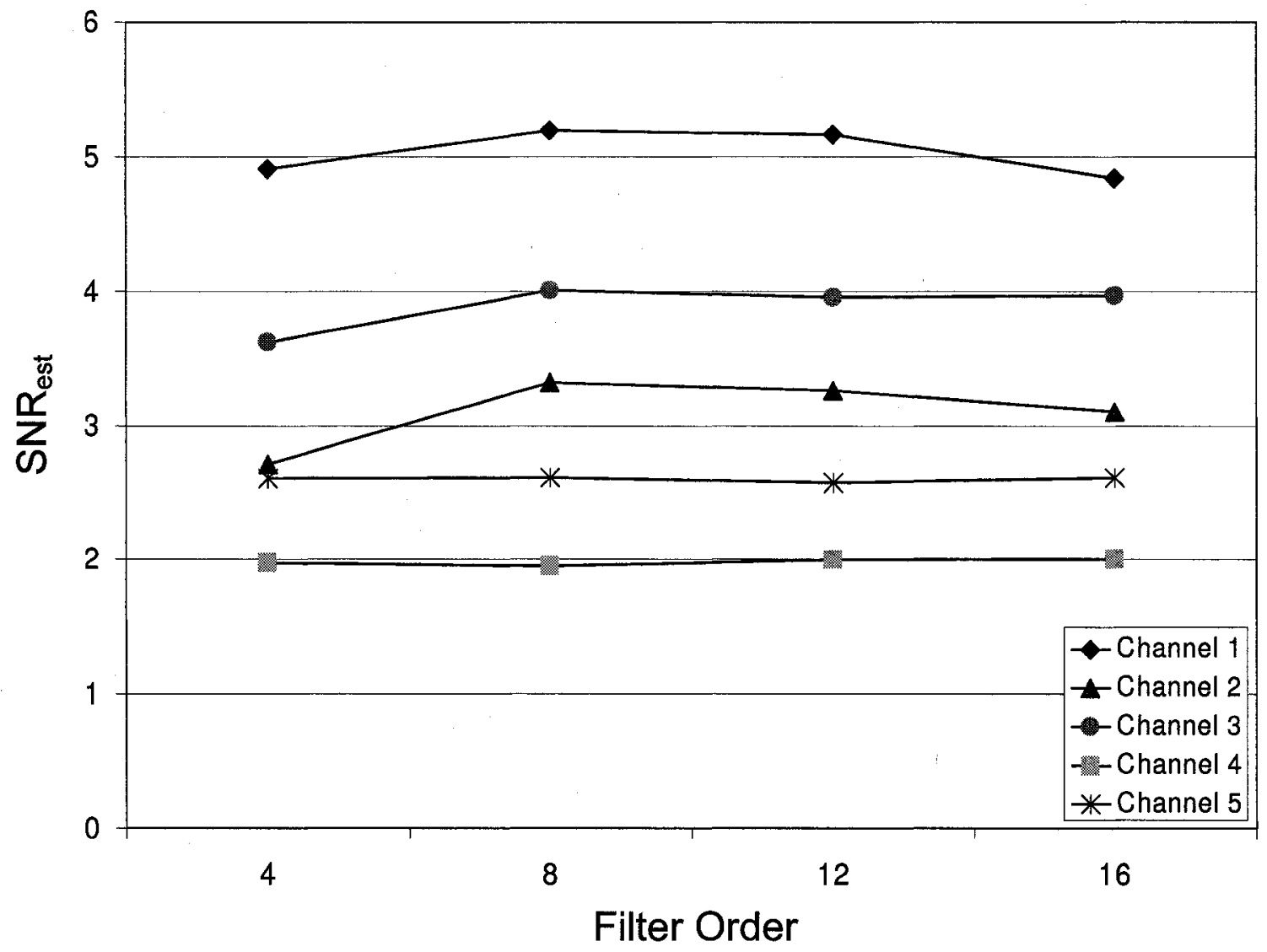

Figure 5-4 SNR $_{\text {est }}$ of abdominal channels after TANC for various filter orders using triggering with linear transitions

It was found that an adaptive filter using triggering with on/off transitions would not extract the FECG in one pass when filter orders greater than 28 were used. Similarly, triggering with linear transitions was restricted to filter orders 16 and smaller. Although restricted to a maximum filter order, the stage 1 results using TANC with a filter order of 8 have performed equal to or better than, albeit by a small amount, the results given without triggering. It is believed that there is still room for improvement in the results using TANC. For instance, higher filter orders can be applied without the need for multiple passes through 
the adaptive filter by using input signals with longer durations. Further parameter optimization may also provide better results.

For easier comparison, the optimal results using one pass are displayed in Figure 5-5. Channel 1 provided the highest $\mathrm{SNR}_{\text {est }}$ result and will be used as the template model for TASI in stage 2.

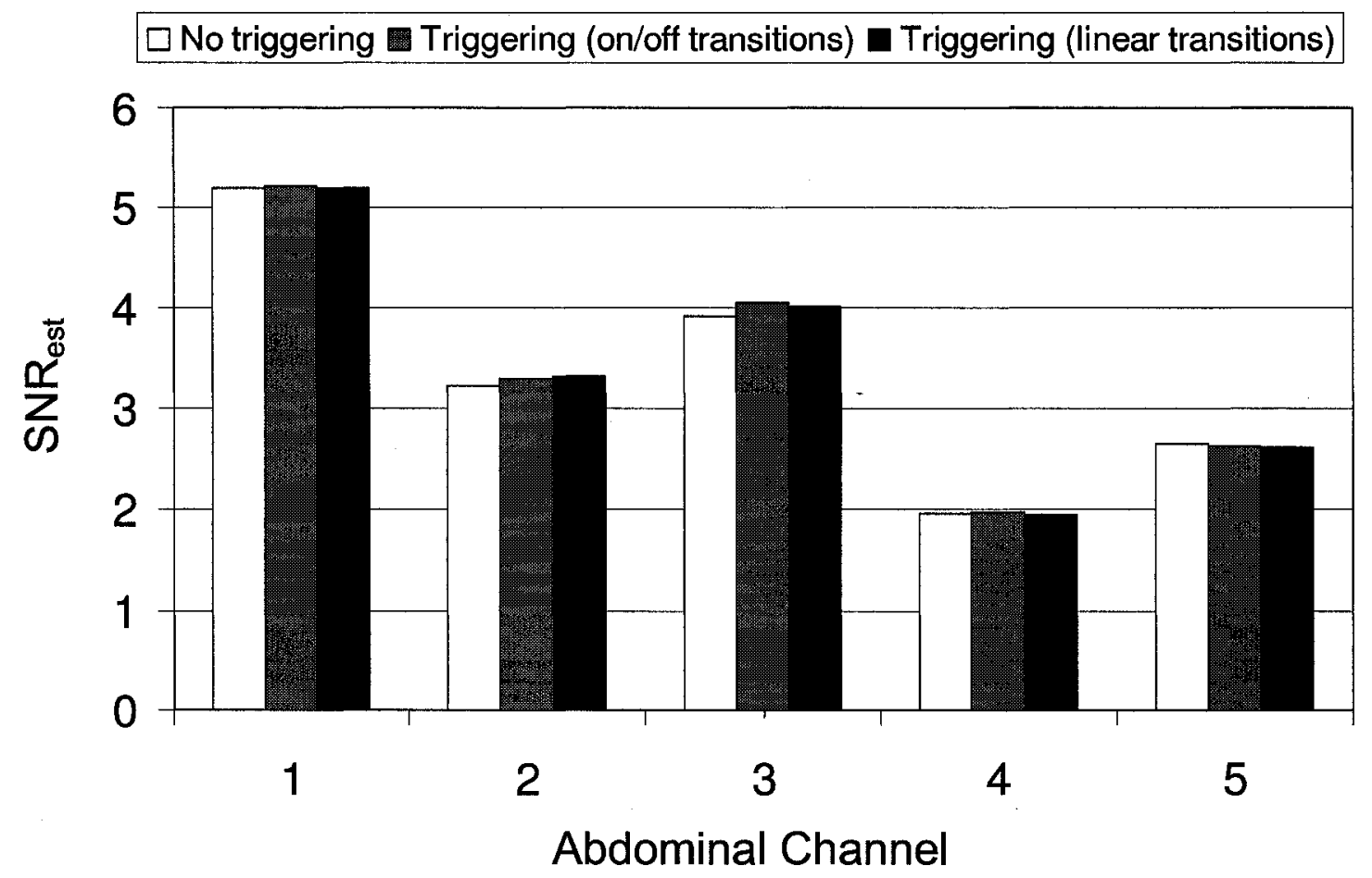

Figure 5-5 SNR $_{\text {est }}$ of abdominal channels after TANC for various triggering modes

Figure 5-6 displays a segment of the system output for channel 1 following TANC using various triggering modes to give a visual representation of the extracted FECG. The FECG is now the dominating signal, however remnants of the MECG remain at $0.65 \mathrm{~s}$ and $1.40 \mathrm{~s}$. Once again, as explained in Section 4.5.1, any improvement in accuracy provided by 110 
triggering cannot be expected to be seen visually at this stage. We base our conclusions on the quantifiable results.

(a) No Triggering
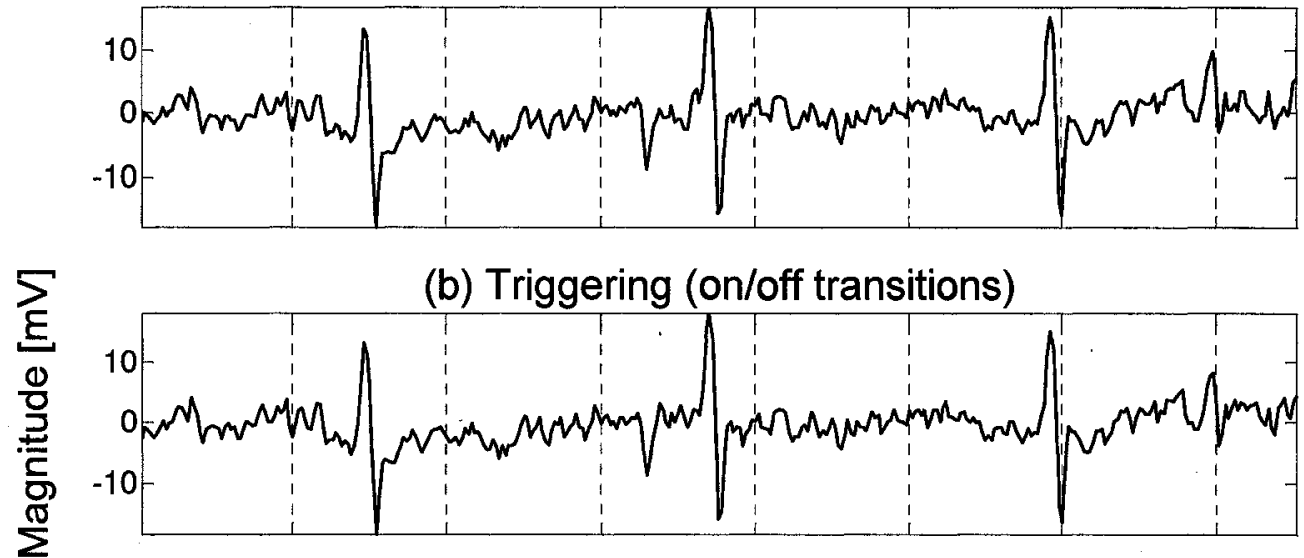

(c) Triggering (linear transitions)

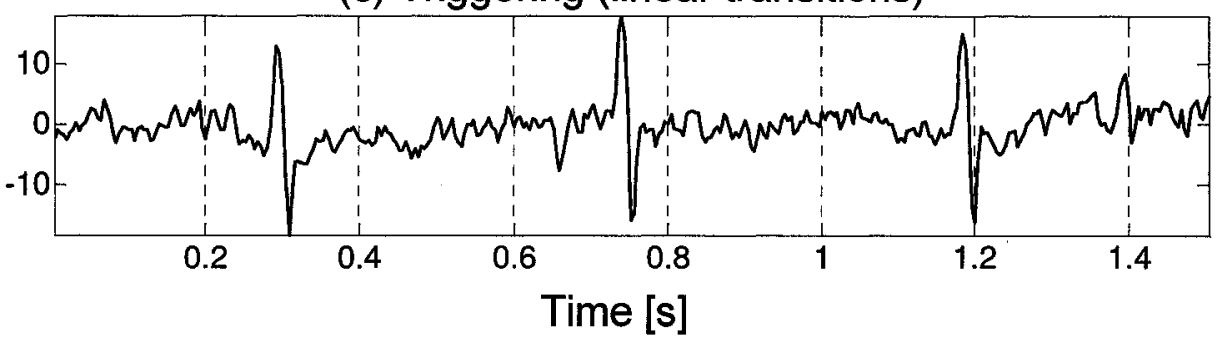

Figure 5-6 Estimated FECG after TANC various triggering modes using channel 1

\subsubsection{Stage 2 Results}

Similar to stage 1 , initial simulations for stage 2 were run using various filter orders to investigate an optimal filter order. The optimal results of stage 1 were chosen for the inputs to the second stage. In the case of no triggering, this was 28 , and in the case of triggering (both with on/off transitions and with linear transitions), this was 8 . Again, the RLS adaptive algorithm with adaptive memory (i.e., time-varying forgetting factor $\lambda$ ) was used in our simulations in an FIR filter. An initial $\lambda$ of 0.999 with time-varying values 
between 0.999 and 0.9999 (increments of 0.0001) were used. Abdominal channel 1 was used as the template model for the primary input to the ASI scheme, since this channel provided the highest SNR $_{\text {est }}$ after stage 1.

Figure 5-7 displays the results after stage 2 for varying filter orders and triggering modes. In all triggering modes, the optimal filter order for TASI was empirically found to be 8. Using no triggering, there was an $\mathrm{SNR}_{\text {est }}$ increase of 3.86 (from 5.20 to 9.06 ). Better results were provided using triggering, with an $\mathrm{SNR}_{\text {est }}$ increase of 4.65 (from 5.22 to 9.87 ) and 4.35 (from 5.20 to 9.55 ) with on/off transitions and linear transitions, respectively.

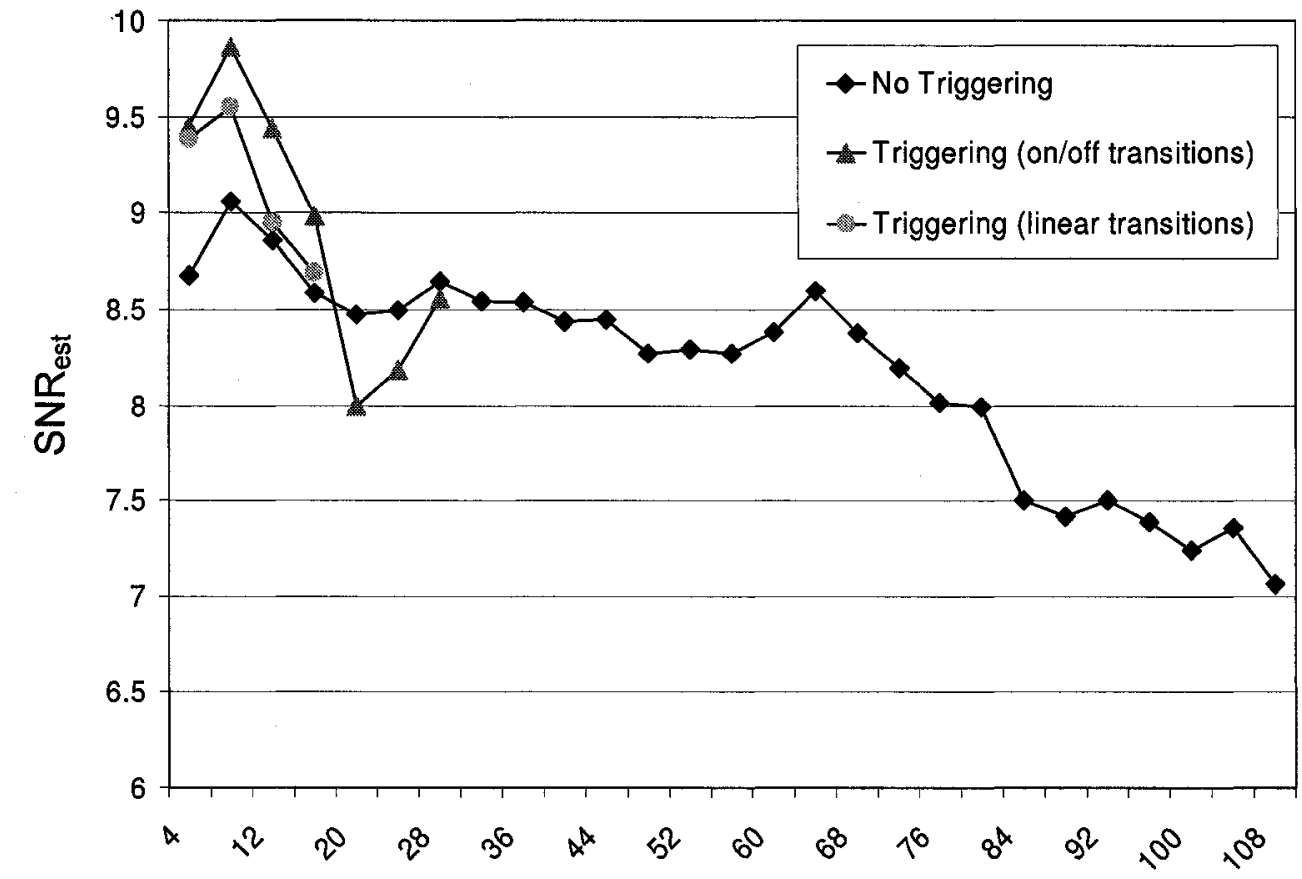

Filter Order

Figure 5-7 SNR $_{\text {est }}$ of final FECG estimate after TASI for various filter orders and triggering modes 
For better comparison, the optimal $\mathrm{SNR}_{\text {est }}$ results are displayed in Figure 5-8, along with the results of stage 1 . The use of triggering in the multichannel ASI scheme has allowed further attenuation of noise sources in the extracted FECG data. Figure 5-9 displays a segment of the final FECG estimate, overlapped with the estimate after stage 1, showing the reduction in noise for the various triggering modes.

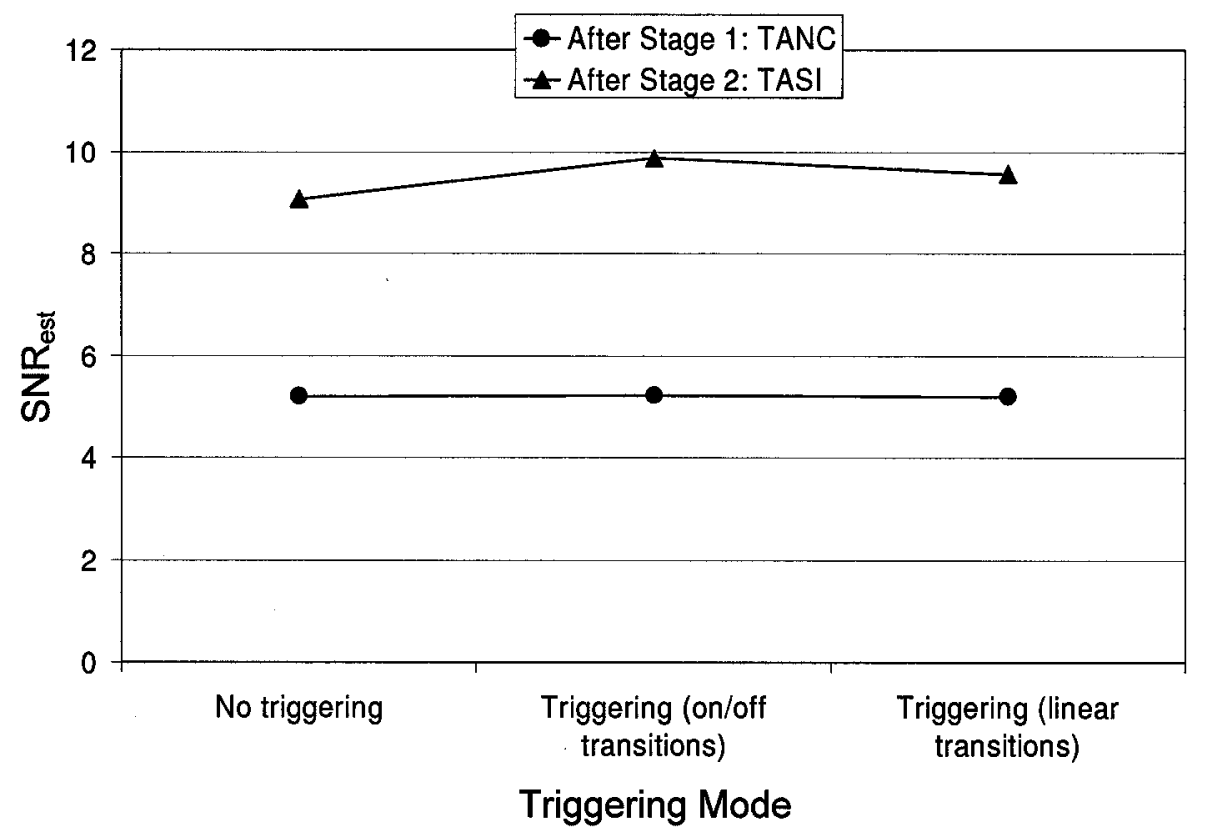

Figure 5-8 SNR $_{\text {est }}$ of final estimate after TASI for various triggering modes 
(a) No Triggering -After stage 1: TANC
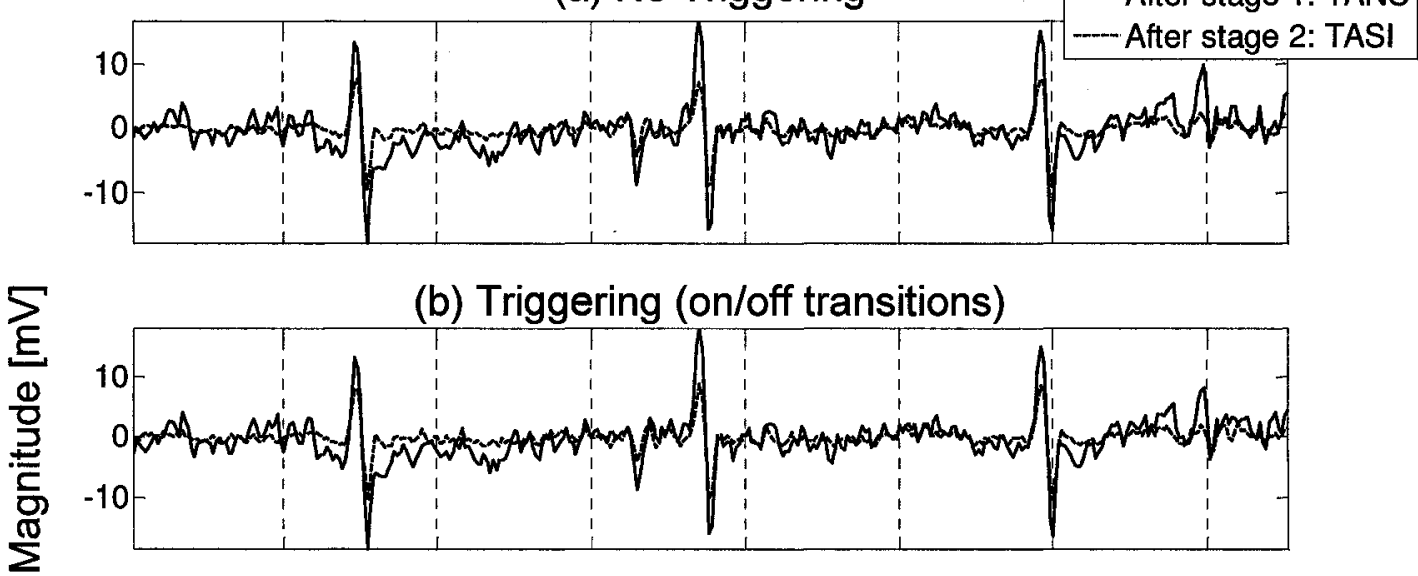

(c) Triggering (linear transitions)

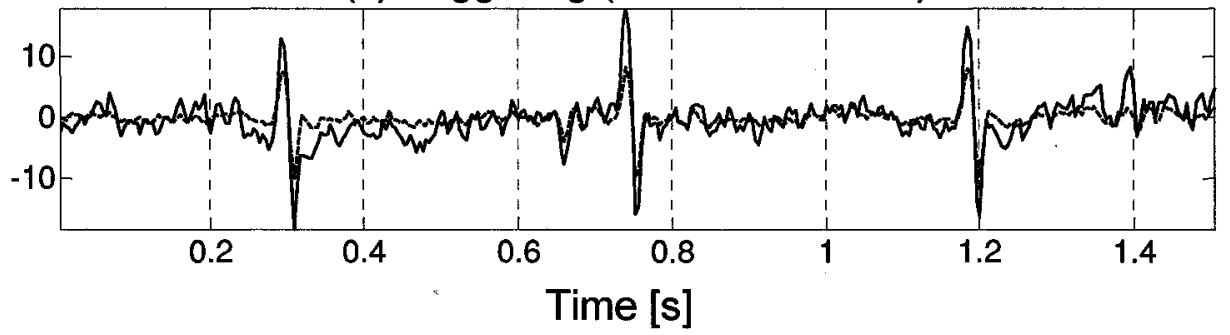

Figure 5-9 Estimated FECG after TANC and TASI for various triggering modes

\subsection{Conclusions}

In this chapter, the two-stage multichannel adaptive signal extraction and enhancement scheme was applied to real MECG and FECG data. Stage 1 results indicate equal or better performance with the use of triggering compared to no triggering, with $\mathrm{SNR}_{\text {est }}$ results for the template channel of $5.20,5.22$ and 5.20 for no triggering, triggering with on/off transitions and triggering with linear transitions, respectively. Stage 2 results confirm the applicability of triggering to ASI. The reference input was chosen as channel 1 since it provided the highest $\mathrm{SNR}_{\text {est }}$ results. The use of no triggering $\left(\mathrm{SNR}_{\text {est }}=9.06\right)$ was 
outperformed by both triggering modes, with final SNR $_{\text {est }}$ results of 9.87 and 9.55 for on/off transitions and linear transitions, respectively. As opposed to the results of Chapters 3 and 4, triggering with on/off transitions has outperformed triggering with linear transitions in this chapter. This demonstrates that the triggering method itself can be quite varied in its abilities.

It was found that an adaptive filter using triggering requires input data lengths greater than that required with triggering. This is due to the fact that adaptation is not always enabled, and so the adaptive algorithm requires more samples to converge. Unfortunately, the short data length that was available for the simulations in this study presented this problem. Simulations using triggering were restricted to smaller filter orders to compensate for this. It is believed that there is still room for improvement in the results using triggered adaptive filtering. The limitations imposed can be solved by access to data with longer duration, further optimization of the triggered adaptive filter parameters, or by running the data through the filter multiple times to allow convergence. The latter technique would introduce additional gradient noise to the estimate; however, this may be remedied using post low-pass filtering.

Although triggered adaptive filtering was limited in its improvements due to the need for a longer data set, and thus was not fully optimized in the simulations of this chapter, the results are still encouraging and confirm, using real data, that the use of triggering can be advantageous for extraction and enhancement of the FECG. 


\section{Chapter 6 \\ Conclusions}

\subsection{Summary}

The objective of this thesis has been to investigate a novel FECG extraction and enhancement technique using linear adaptive filtering with triggering, based on noninvasive abdominal measurements. In Chapter 3, we have proposed to use a TANC system for the extraction of the FECG from a noninvasively acquired abdominal composite signal containing a dominant interfering MECG component. This can be realizable through the use of an extra modality, such as the FPCG, that can be used as an adapt-disable trigger. Due to the intrinsic temporal correlation between the heart's mechanical and electrical activity, the FPCG can be used to derive the temporal occurrence of FECG pulses. By preventing adaptation during the FECG pulse, we have shown that the adaptation process arrives at a more accurate result than with classic ANC. The results were quantified using the PPRD performance measure. An improvement of $3.80 \%$ PPRD using triggering with on/off transitions compared to no triggering is attained. Using linear transition weighted triggering, the PPRD of the FECG estimate was found to be $5.49 \%$ better than without triggering.

Although the use of weighted triggering has improved the estimate of the tissue filter resulting in a better estimate of the FECG, the technique is limited by the SNR of the input. This is addressed in Chapter 4, where we have proposed an adaptive two-stage multichannel signal extraction and enhancement scheme. This scheme was implemented in a linear FIR 
filter using the RLS adaptation algorithm. An external SP, which was applied to a subject's back, was acquired from the subject's front using multiple abdominal surface electrodes. This was used to represent an FECG with a controllable amplitude. Each acquired abdominal signal is a composite signal containing a dominant ECG from the subject as well as the tissue-filtered SP. The SP was then extracted from the multiple composite signals using TANC and enhanced using TASI. SNR est $_{\text {and }}$ PRD est $_{\text {were }}$ used as secondary performance measures indicating the degree of attenuation of the interferences, which include the subject's ECG and random noise. Results of stage 1 simulations show similar $\mathrm{SNR}_{\mathrm{est}}$ and $\mathrm{PRD}_{\mathrm{est}}$ results across the various triggering modes (no triggering, triggering with on/off transitions, and triggering with linear transitions). With an applied SP amplitude of $20 \mathrm{~V}$, triggering with on/off transitions gave an increase in $\mathrm{SNR}_{\mathrm{est}}$ of $0.04\left(\mathrm{SNR}_{\mathrm{est}}=3.24\right)$ and a $\mathrm{PRD}_{\text {est }}$ improvement of $1.6 \%\left(\mathrm{PRD}_{\mathrm{est}}=56.5 \%\right)$ compared to $\mathrm{ANC}$ without triggering, while triggering with linear transitions gave the same $\mathrm{SNR}_{\text {est }}$ as no triggering $\left(\mathrm{SNR}_{\text {est }}=3.20\right)$ and a $\mathrm{PRD}_{\text {est }}$ improvement of $1.6 \%\left(\mathrm{PRD}_{\mathrm{est}}=56.5 \%\right)$.

In stage 2, we attempt to further increase the SNR of the estimate by exploiting the multiple channels using a TASI scheme. The reference input to this scheme was the estimated signal output from stage 1 with the best performance to be used as a template. The stage 2 results indicate better performance using triggering. An SNR est $_{\text {improvement of } 2.61}$ was provided by triggering with linear transitions $\left(\mathrm{SNR}_{\mathrm{est}}=9.83\right)$ over no triggering $\left(\mathrm{SNR}_{\mathrm{est}}\right.$ $=7.22$ ) with an applied SP amplitude of $20 \mathrm{~V}$. Similarly, a PRD est $_{\text {improvement of } 3.1 \% \text { was }}$ achieved. It is realized, however, that in order for an $\mathrm{SNR}_{\mathrm{est}}$ improvement to be achieved, a threshold SNR $_{\text {est }}$ level must be met for the inputs to the TASI scheme. This threshold $\mathrm{SNR}_{\text {est }}$ 
was shown to be lower when triggering is used, indicating a more robust system that can handle inputs with lower SNRs compared to ASI without triggering.

In Chapter 5, the two-stage multichannel adaptive FECG extraction and enhancement scheme was applied to real MECG and FECG data. Results were analyzed using the $\mathrm{SNR}_{\text {est }}$ secondary performance measure. Stage 1 results indicate equal or better performance provided with the use of triggering, albeit by a small amount, with $\mathrm{SNR}_{\text {est }}$ results for the template channel of 5.20, 5.22 and 5.20 for no triggering, triggering with on/off transitions and triggering with linear transitions, respectively. Stage 2 results confirm the applicability of triggering to ASI. The use of no triggering $\left(\mathrm{SNR}_{\mathrm{est}}=9.06\right)$ was outperformed by both triggering modes, with final $\mathrm{SNR}_{\text {est }}$ results of 9.87 and 9.55 for on/off transitions and linear transitions, respectively. As opposed to the results of Chapters 3 and 4, triggering with on/off transitions has outperformed triggering with linear transitions in this chapter. This demonstrates that the triggering method itself can be quite varied in its abilities. Although triggered adaptive filtering was limited in its improvements due to the need for a longer data set, and thus was not fully optimized in the simulations of this chapter, the results are still encouraging and confirm, using real data, that the use of triggering can be advantageous for extraction and enhancement of the FECG.

\subsection{Future Research}

1. The key concept driving the application of triggering to adaptive filtering schemes for FECG extraction is that one can take advantage of an additional modality, such as the FPCG, to estimate the temporal locations of the FECG pulses. This would allow for 
real-time clinical use. Although the timing relationships between adult PCGs and ECGs are well defined in literature, the author found no such relationship specific to fetal signals. It is important to define the general timing relationship between the FPCG and FECG in order to make use of the additional sound modality.

2. In this study, access to real thoracic MECG data simultaneously acquired with abdominal composite ECG data was limited to an online database of a single subject. To verify the general applicability of triggered adaptive filtering for FECG extraction and enhancement, it is imperative to test the system on a larger subject set.

3. In Chapter 3, results given using MECG adapt-enable triggering and dual triggering were hindered by the nature of the synthetic ECG data. It is suggested that future work could involve further analysis of these modes of triggering using real data. This is especially important for the dual triggering mode, since it takes advantage of FECG pulses that do not severely overlap with the MECG, and could thus theoretically provide more accurate FECG estimates than with FECG adapt-disable triggering only.

4. In this study, the results given by using triggering with on/off transitions versus those given by linear transitions were varied, and offered no conclusion for which transition type is more suitable. The reason for this phenomenon is not understood, and this suggests that a further study into the mathematical implications of the various 
triggering transitions is required. Furthermore, there are many weighted transition types that have yet to be explored.

5. The focus of this work is to establish the usefulness of triggering in the ANC and ASI schemes; however many other algorithms exist for FECG extraction and enhancement, such as BSS, neural networks and temporal averaging of the FECG. Extension of the concept of triggering and weighted transitions to other algorithms would be an interesting next step. 


\title{
Appendix A
}

\section{Carleton University Research Ethics Committee Consent Form}

\author{
Date of Ethics Approval: April 19, 2005 \\ Biological Signal Research \\ Carleton University \\ Department of Systems and Computer Engineering
}

\section{INFORMED CONSENT}

$\mathrm{I}$, have been invited by Dr. Adrian Chan, and his research associates, of the Department of Systems and Computer Engineering at Carleton University, Ottawa, ON to participate in a study on biological signals.

The purpose of the study is to examine various biological signals including: electrocardiogram (ECG) - heart signal, myoelectric signal (MES) - muscle signals, electroneurogram (ENG) - nerve signals, and electroencephalogram (EEG) - brain signals.

These signals will be monitored non-invasively using electrodes on the skin surface. Although this is a non-invasive measuring technique, these surface signals contain valuable information pertaining to the status of internal organs (e.g. heart, muscles, and nerves).

I understand that surface electrodes will be placed on me to acquire the necessary biological signals. Depending on the study, the number and exact placement of the electrodes will vary. A maximum of 16 simultaneous channels will be used.

\section{[ ] I have been fully informed of the study that I am participating in.}


Given the range of experiments I understand that I am being asked to participate in:

[ ] ECG study that requires the application of surface electrodes and an external stimulus.

The risk involved is a slight irritation of the skin under the electrode sites. One channel may also be used to apply an external electrical stimulus, which is felt as a slight shock to the applied area. The amplitude and length of the stimulus will be carefully controlled by the experimenter.

[ ] neuromuscular study that requires the application of surface electrodes.

The risk involved is similar to that of obtaining an ECG (cardiac) record (i.e. slight irritation of the skin under the electrode site). I may be required to lift a weight, provide a maximum voluntary contraction, or fatigue. I may feel the short-term discomfort of muscle fatigue.

[ ] speech-related studies where a microphone will be used to record my voice.

I may be asked some questions to ascertain some additional data, which may include: age, sex, height, weight, native language, birth place, whether I have any known neuromuscular disorders.

I have been informed in advance that the study session for the data collections will be:

[ ] approximately one hour

[ ] up to three hours.

I may be asked to return for additional sessions, and will be informed of this at the end of the study session. I am in no way obligated to participate in these additional sessions. 
There are no direct benefits or remuneration for my participation in this study.

Should the experimenter note any unusual readings during the course of the experiment the study will be stopped immediately. The experimenter is not a physician and cannot make a medical diagnosis. I will be asked to contact my family physician. The researcher will contact my physician in writing explaining why the experiment was stopped. I may not return to the study or undertake any further experiments without the written consent of my physician.

I understand that the data from this research will be used in scientific reports, presentations, and publications and my identity will remain confidential. Data will be kept electronically and my consent forms and information will be kept as a hardcopy. Access to the data will be restricted to the researcher investigators. Data may be kept for an indefinite period of time. Note data may be shared with other investigators (possibly at other institutions) for research purposes.

My identity will be kept strictly confidential unless otherwise discussed with the researcher. Any scientific report, presentation, or publication of the data will refer to me using a subject number. Information on my sex, age, and native language may be used.

Participation in this study is strictly voluntary. I am free to withdraw from the experiment at any time and without any consequences. I will also declare if the researcher can/cannot use the data I have provided should I decide to withdraw from the study.

This study has been reviewed and received ethics clearance though the Carleton University Research Ethics Committee in accordance to the Tri-Council Policy Statement for Ethical Conduct for Research Involving Humans. Should I have any concerns or questions about my involvement in this study I may contact the committee chair: 
Professor Klaus Pohle, Chair

Research Ethics Committee

613-520-2600 ext. 7434

klaus_pohle@carleton.ca).

I may contact one of the investigators for consultation of any concerns I have about the research. In addition, I may also request any publications and information about the final results or conclusions from the study:

Dr. Adrian Chan

Department of Systems and Computer Engineering

Carleton University

1125 Colonel By Drive

Ottawa ON K1S 5B6 CANADA

(613) 520-2600 ext. 1535

Signature: Date:

Experimenter:

Signature:

Date:

Research undertaken as part of a graduate or undergraduate course requirement: YES / NO

If YES specify course name and course number

Research undertaken as part of a graduate or undergraduate thesis research: YES / NO 


\section{Consent for Photographs}

I understand that photographs (conventional/digital) may be required to document portions of the study. I have the option to consent or decline the usage of photographs during the study. Usage of these photographs will be restricted to scientific reports, presentations, or publications. I understand that my confidentiality will be maintained as best as possible; however, the usage of photographs may compromise my confidentiality.

Photographs will be required in this study YES / NO

If YES photographs will be CONVENTIONAL / DIGITAL

I, _ hereby consent to having photographs taken.

Signature:

Date:

Experimenter:

Signature:

Date: 


\section{References}

[1] R. C. Goodlin, "History of fetal monitoring", Am. J. Obstet. Gynecol., vol. 133, no. 3, pp. 323-352, Feb. 1979.

[2] R. K. Freeman, T. J. Garite and M. P. Nageotte, Fetal Heart Rate Monitoring, 3rd ed., Philadelphia, PA: Lippincott Williams \& Wilkins, 2003.

[3] D. Callaerts et al, "Acquisition and processing of the antepartum FECG", H. Van Geijn, Ed., F. Copray, Ed., A Critical Appraisal of Fetal Surveillance, Elsevier Science B.V., pp. 371-380, 1994.

[4] S. W. D'Souza, P. Black, T. Macfarlane and B. Richards, "Fetal scalp damage and neonatal jaundice: a risk of routine fetal scalp electrode monitoring", J. Obstet. Gynecol., vol. 2, pp. 161-164, 1982.

[5] A. C. Deans and P. J. Steer, "The use of the fetal electrocardiogram in labour", J. Obstet. Gynecol., vol. 101, pp. 9-17, Jan. 1994.

[6] J. P. Neilson, "Fetal electrocardiogram (ECG) for fetal monitoring during labour", The Cochrane Database of Systematic Reviews, issue 2, 2003.

[7] B. H. Brown et al, Medical Physics and Biomedical Engineering, Philadelphia, PA: Institute of Physics Pub., 1999.

[8] M. Akay, Ed., Time Frequency and Wavelets in Biomedical Signal Processing, Piscataway, NJ: IEEE Press, 1998.

[9] J. G. Webster, Ed., Medical Instrumentation: Application and Design, 3rd ed., NY: John Wiley \& Sons Inc., 1998.

[10] Loma Linda University School of Medicine, (C) San Diego State University, "Heart sounds", http://www.sci.sdsu.edu/multimedia/heartsounds/, last visited Nov. 2006.

[11] K. L. Fernando et al, "Robust estimation of fetal heart rate variability using Doppler ultrasound", IEEE Trans. Biomed. Eng., vol. 50, no. 8, pp. 950-957, Aug. 2003. 
[12] C. H. L. Peters et al, "Beat-to-beat detection of fetal heart rate: Doppler ultrasound cardiotocography compared to direct ECG cardiotocography in time and frequency domain", Physiol. Meas., vol. 25, pp. 585-593, 2004.

[13] H. F. Ruth, "Doppler Ultrasound: The ability to measure and image blood flow", IEEE Eng. Med. Biol., pp. 31-40, Nov./Dec. 1996.

[14] J. Jezewski et al, "Coping with limitations of Doppler ultrasound fetal heart rate monitors", Proc. RC-IEEE-EMBS \& 14th BMESI, 1995.

[15] B. Widrow et al, "Adaptive noise cancelling: Principles and applications", Proc. IEEE, vol. 63, pp. 1692-1716, Dec. 1975.

[16] E. R. Ferrara and B. Widrow, "The time-sequenced adaptive filter", IEEE Trans. Acoust., Speech, Signal Processing, vol. ASSP-29, no. 3, pp. 679-683, Jun. 1981.

[17] E. R. Ferrara, “The time-sequenced adaptive filter", $P h$. D. dissertation, Stanford University, Stanford, CA, Dec. 1977.

[18] E. R. Ferrara and B. Widrow, "Fetal electrocardiogram enhancement by time-sequenced adaptive filtering", IEEE Trans. Biomed. Eng., vol. 29, no. 6, pp. 458-460, Jun. 1982.

[19] B. Widrow and M.E. Hoff Jr., "Adaptive switching circuits", IRE WESCON Conv. Rec., Pt. 4, pp. 96-104, 1960.

[20] S. Haykin, Adaptive Filter Theory, 4th ed., Upper Saddle River, NJ: Prentice Hall, 2002.

[21] L. D. Lathauwer, B. De Moor, and J. Vandewalle, "Fetal electrocardiogram extraction by blind source subspace separation", IEEE Trans. Biomed. Eng., vol. 47, no. 5, pp. 567572 , May 2000.

[22] V. Zarzoso and A. K. Nandi, "Noninvasive fetal electrocardiogram extraction: Blind separation versus adaptive noise cancellation", IEEE Trans. Biomed. Eng., vol. 48, no. 1, pp. 12-18, Jan. 2001. 
[23] M. G. Jafari and J. A. Chambers, "Fetal electrocardiogram extraction by sequential source separation in the wavelet domain", IEEE Trans. Biomed. Eng., vol. 52, no. 3, pp. 390-400, Mar. 2005.

[24] R. Plonsey, Bioelectric Phenomena, NY: McGraw-Hill, 1969.

[25] V. Zarzosa and A.K. Nandi, "Blind source separation" in Blind Estimation Using Higher-Order Statistics, A.K. Nandi, Ed., Boston, MA: Kluwer Academic, pp. 167-252, 1999.

[26] R. Ortiz et al, "Differences in foetal heart rate variability from phonocardiography and abdominal electrocardiography", J. Med. Eng. Tech., vol. 26, no. 1, pp. 39-45, Jan./Feb. 2002.

[27] R. K. Freeman, T. J. Garite and M. P. Nageotte, Fetal Heart Rate Monitoring, 3rd ed., Philadelphia, PA: Lippincott Williams \& Wilkins, 2003.

[28] R. M. Rangayyan, Biomedical Signal Analysis - A Case Study Approach, NY: WileyInterscience, 2002.

[29] J. Wartak, Phonocardiology: Integrated Study of Heart Sounds and Murmurs, NY: Harper \& Row, 1972.

[30] S. Suppappola and Y. Sun, "Nonlinear transforms of ECG signals for digital QRS detection: A quantitative analysis”, IEEE Trans. Biomed. Eng., vol. 41, no. 4, pp. $397-$ 400, Apr. 1994.

[31] M. J. Burke and M. Nasor, "The time relationships of the constituent components of the human electrocardiogram", J. Med. Eng. Tech., vol. 26, no. 1, pp. 1-6, Jan./Feb. 2002.

[32] P. E. McSharry et al, "A dynamical model for generating synthetic electrocardiogram signals", IEEE Trans. Biomed. Eng., vol. 50, no. 3, pp. 289-294, Mar. 2003.

[33] A. Alshamali and A. S. Al-Fahoum, Comments on "An efficient coding algorithm for the compression of ECG signals using the wavelet transform", IEEE Trans. Biomed. Eng., vol. 50, no. 8, pp. 1034-1037, Aug. 2003. 
[34] A. S. Al-Fahoum, "Quality assessment of ECG compression techniques using a waveletbased diagnostic measure", IEEE Trans. Inf. Technol. Biomed., vol. 10, no. 1, pp. 182191, Jan. 2006.

[35] J. Vandewalle, Database for the Identification of Systems (DAISY), C SISTA, "Cutaneous potential recordings of a pregnant woman", data set [96-012], http://homes.esat.kuleuven.be/ smc/daisy/daisydata.html, last visited Nov. 2006.

[36] D. Callaerts et al, "Description of a real-time system to extract the fetal ECG", Clin. Phys. Physiol. Meas., vol. 10, suppl. B, pp. 7-10, 1989. 